


CHECK LIST OF NORTH AMERICAN AMPHIBIANS AND REPTILES 
LONDON : HUMPHREY MILFORD

OXFORD UNIVERSITY PRESS 


\title{
A CHECK LIST OF NORTH AMERICAN AMPHIBIANS AND REPTILES
}

\author{
BY \\ LEONHARD STEJNEGER \\ AND \\ THOMAS BARBOUR
}

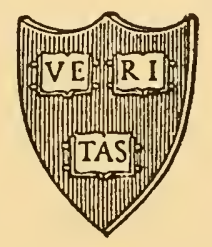

THIRD EDITION

CAMBRIDGE

HARVARD UNIVERSITY PRESS

1933 
COPYRIGHT, 1933

BY THE PRESIDENT AND FELLOWS OF HARVARD COLLEGE

PRINTED AT THE HARVARD UNIVERSITY PRESS

CAMBRIDGE, MASS., U.S. A. 


\section{INTRODUC'TION 'TO THIRD EDITION}

7 THE second edition of the Check List, which appeared in 1 1923, is now out of print, and the great volume of additions to our knowledge of American reptiles and amphibians has been used in preparing this third edition.

Many colleagues have aided us with helpful criticism and suggestions. It is impossible to mention the names of all with whom we have corresponded, but some have been of such great and constant assistance that they should be especially remembered. Our hearty thanks are particularly due to Mrs. H. T. Gaige, Professor E. R. Dunn, Mr. L. M. Klauber, Mr. K. P. Schmidt, and Dr. Frank N. Blanchard, and also to Messrs. C. L. Walker, O. C. Van Hyning, and G. M. Netting.

It may perhaps be well to restate here the spirit by which we have been guided in preparing this list. In general we have been inclined to admit all forms described since the last list unless they have been shown publicly to be untenable or unless we have a decisive opinion of our own or some other sufficient reason for rejecting them. Doubtful names may be included in the Check List for debate or as an index to literature. In the use of binominals and trinominals we feel inclined to exercise our own discretion. We are opposed to mixing the biological idea of a subspecies with the convenience or inconvenience of a trinominal designation. We hold a subspecies to be a form which is manifestly descended from another form. If they are still forms connected by intergrades we accept trinominals unless the percentage of intergrades is so large that it becomes unprofitable so to do. But if there is reason to believe that the connection between two forms has once been broken and that the intergrades now recorded are either the result of hybridization or individual reversion, we see no gain in treating them trinominally, par- 
ticularly in those cases where each form may, in its own turn, normally intergrade with other trinominals. We feel inclined to conserve the status of the names of the old list except when absolutely compelled by the facts to make a change.

In some respects we may seem over-inclined to be thus conservative. Dr. Dunn has recently proposed that the genus Tropidoclonion be merged with Thamnophis. But since the monotypic form is obviously more different from any species of 'Thamnophis than any of the species in that genus are one from another we prefer still to recognize the peculiar genus for this single species. Considering, as we do, that genera are groups for convenience, that no two people are ever likely to agree for long on generic limitations, we feel that any means of breaking up large genera by setting off particularly well differentiated species or groups of species is justifiable and praiseworthy. The recent attempt of Myers (Proc. Biol. Soc. Wash., Vol. 43, March 12, 1930, p. 61) to set up the name Bufo canagicus (Pallas) for Bufo boreas has not seemed to us in the least convincing, and we are entirely in agreement to ignore this name since it is impossible from Pallas' description to know whether he had a Bufo or a Rana in hand. ${ }^{1}$

Our hearty thanks are due to Mr. and Mrs. R. E. Bowen and Miss H. M. Robinson for their care in verifying references and assisting in the preparation of the manuscript.

T. B.

\footnotetext{
1 It appears at the last minute, after printing of the text has been completed, that two names have been inadvertently omitted:

Elaphe lindheimeri Stejneger and Barbour (Check List No. Amer. Amph. Rept., 2nd Ed., 1923 , p. 92) should appear as a synonym of Elophe obsoleta confinis, p. 99.

Chilomeniscus ephippicus Stejneger and Barbour (Check List No. Amer. Amph. Rept., 2nd Ed., 1923, p. 102) should appear as a synonym of Chilomeniscus cinctus, p. 114.
} 


\section{INTRODUCTION TO SECOND EDITION}

The first edition of the Check List seems to have been useful. Great changes have come in our knowledge of the North American fauna since it was written, so great indeed that there has been a general request for a revised edition. This we have attempted in as impartial a manner as possible. Probably no one will agree with all our conclusions. The reason for issuing this revision now is that the present seems to be as good a time as any later date, in view of the fact that the last word will not be said for years to come.

Recent writers have often erred egregiously in the use of accents in Spanish place names. We confess it is difficult to satisfy the puristic conventions. Our general rule is to accent all Spanish words in which the last syllable is stressed and which end in a vowel.

It is not the real province of a Check List to initiate changes in usage except where nomenclature does not conform to the International Code. We admit new species and subspecies as they are proposed, except where their validity is definitely contra-indicated. As to the various more fundamental changes in classification which have been proposed we can only say that we are inclined to consider them very sympathetically, but they have not as yet stood the test of time. We have, however, omitted the term Eublepharidae most willingly. But Pseudacris and Manculus, which some would suppress, are convenient names for apparently natural associations of species, and, therefore, who would ask more reason for their retention in the ranks of valid genera? It is to be hoped that American herpetology may not fall into the unhappy condition now seen in our ornithology with its overmultiplied monotypic genera: still, however, any reasonable efforts to break up the great unwieldy genera are certainly welcome. 
Thus without special aim to be consistent we have taken up the various problems presented by recent workers, feeling generally that it is our duty to record established custom rather than to initiate or to follow hastily each proposed change.

Thanks are due to several of our North American coworkers for advice and assistance. We would mention especially Dr. Emmett Reid Dunn, Dr. Alexander G. Ruthven, and Mr. Karl P. Schmidt.

T. B. 


\section{INTRODUCTION TO FIRST EDITION}

A Check List of North American Amphibians and Reptiles has long been wished for by all students of American herpetology. This list has been prepared generally upon the lines of the American Ornithologists' Union Check List of Birds, and, following that example, it has included the species and subspecies which the authors deem valid ${ }^{1}$ and of certain occurrence in North America, north of the Rio Grande, and in Lower California, Mexico. Miller's List of North American Land Mammals (Bull. U. S. Nat. Mus., No. 79, 1912) has also been a valued guide.

This volume is the result of lists begun some years ago independently by both authors. The arrangement is on the following system: The higher groups and genera are in systematic sequence, the species are in alphabetic order. No attempt is made to give synonyms. Species not listed are believed to be invalid. The citation of original appearance of names is given for all except such (e. g., family names) as are formed automatically. Following the citation of the original description of each species comes the place of first appearance of the name in its present combination - accurate in so far as we have been able to determine. References are added to Cope's North American Batrachia and to his Crocodilians, Lizards, and Snakes of North America. Both are frequently erratic and inaccurate, but large and excellent portions of both works are to a great extent from the pen of S. F. Baird, as painstaking an investigator as this country has produced. These monographs are in widespread use, and constitute the most recent attempts toward complete lists. In the case of

1 By this statement it is not meant that the authors in all cases have verified the status of a number of more recently established forms by critical study. The judgment of reliable workers has been accepted where no special reason appeared to contra-indicate the validity of the form. As for the admission of subspecies - or rather trinominal designation — for certain forms no special attempt has been made at consistency, the authors on principle leaning towards binominals in all cases where the need of trinominals has not been clearly established. 
the difficult genus Thamnophis references are given to Ruthven's excellent review. Common names for reptiles and amphibians are in great confusion, and are often of generic rather than of specific application. No attempt is made to recognize any except those which are in well-established usage. The host which have been recently coined by various writers are frequently inapt and almost surely unlikely to survive. It is entirely possible that some vernacular names really often used have escaped our notice, since many are in restricted local use. The type localities are as nearly exact as it is possible to determine. This cannot be said regarding the ranges. Many are obviously faulty, but a sincere attempt has been made to collect records of authentic captures; however, with a literature so extensive and so scattered, records have almost surely been overlooked. In many cases, our knowledge does not warrant drawing hard and fast lines delimiting a form's occurrence, and we often state ranges in purposely general terms. There still is opportunity for valuable field work to determine the precise distribution of many of our most common species. An example is the recent discovery that Virginia valeriae is far from rare in parts of New Jersey.

Our hearty thanks are due to many for information and advice, especially to Samuel Henshaw, John Van Denburgh, and Alexander G. Ruthven.

G. K. Noble has assisted considerably in verifying references and searching literature, and for this assistance he has our grateful remembrance.

T. B. 


\section{CONTENTS}

CLASS AMPHIBIA . . . . . . . . . . . . . 1

Order Caudata . . . . . . . . . . . . . . 1

Suborder Proterda. . . . . . . . . . . . . . 1

Family Necturidae . . . . . . . . . . . . . 1

Genus Necturus . . . . . . . . . . . . 1

Suborder Mutabilia . . . . . . . . . . . . 2

Family Amphiumidae . . . . . . . . . . . . . . . . z

Genus Amphiuma .. . . . . . . . . . . . . . . . 2

Family Cryptobranchidae . . . . . . . . . . 2

Genus Cryptobranchus ............ . 2

Family Salamandridae . . . . . . . . . . 3

Genus Triturus . . . . . . . . . . . . . . . 3

Family Ambystomidae . . . . . . . . . . . . . . . . 4

Genus Ambystoma . . . . . . . . . . . . . . . . . 4

Genus Dicamptodon . . . . . . . . . . . . 7

Genus Rhyacotriton . . . . . . . . . . 8

Family Plethodontidae . . . . . . . . . . . . . . . 8

Genus Batrachoseps . . . . . . . . . . . . . . . . 8

Genus Hemidactylium . . . . . . . . . . . 10

Genus Plethodon . . . . . . . . . . . . 10

Genus Ensatina . . . . . . . . . . . . 13

Genus Typhlotriton . . . . . . . . . . . 14

Genus Stereochilus . . . . . . . . . . . 14

Genus Typhlomolge . . . . . . . . . . . . . . 14

Genus Gyrinophilus . . . . . . . . . . . 15

Genus Manculus . . . . . . . . . . . . . . . 15

Genus Pseudotriton. . . . . . . . . . . . . 16

Genus Eurycea . . . . . . . . . . . . 17

Genus Hydromantes . . . . . . . . . . . . . . . . 20

Genus Aneides . . . . . . . . . . . . . . . . . . 20

Genus Desmognathus . . . . . . . . . . . 21

Genus Leurognathus . . . . . . . . . . . . . . . 23

Suborder Meantes . . . . . . . . . . . . . 23

Family Sirenidae . . . . . . . . . . . . . 23

Genus Siren . . . . . . . . . . . . . . 23

Genus Pseudobranchus . . . . . . . . . . . 24

Order Salientia . . . . . . . . . . . . . 25

Suborder Costata . . . . . . . . . . . . . 25

Family Ascaphidae . . . . . . . . . . . . . . 25

Genus Ascaphus . . . . . . . . . . . . . 25

Suborder Linguata : . . . . . . . . . . . . . 25

Family Scaphiopodidae . . . . . . . . . . . . 25

Genus Scaphiopus . . . . . . . . . . 25

$$
42682
$$


Family Bufonidae . . . . . . . . . . . . 26

Genus Bufo . . . . . . . . . . . . . . . . 26

Family Hylidae . . . . . . . . . . . . . 30

Genus Acris . . . . . . . . . . . . . . . . . . 30

Genus Pseudacris. . . . . . . . . . . . . . 31

Genus Hyla . . . . . . . . . . . . . . . . . 33

Family Leptodaetylidae . . . . . . . . . . 36

Genus Leptodactylus . . . . . . . . . . . . . . 36

Genus Eleutherodactylus . . . . . . . . . 37

Genus Syrrhophus . . . . . . . . . . . 38

Family Ranidae . . . . . . . . . . . . . . . . . 38

Genus Rana . . . . . . . . . . . . . . . . 38

Family Brevicipitidae . . . . . . . . . . . . 43

Genus Gastrophryne . . . . . . . . . . . . . . 43

Genus Hypopachus . . . . . . . . . . . . . . . . 43

CLASS REP'TILIA . . . . . . . . . . . . . . . . 44

Subcllass Diapsida . . . . . . . . . . . . . . 44

Order Loricata. . . . . . . . . . . . . . . . . . . . 44

Family Crocodylidae . . . . . . . . . . . . . 44

Genúus Crocodylus . . . . . . . . . . . . . . 44

Genus Alligator . . . . . . . . . . . . . 44

Order Squamata . . . . . . . . . . . . 45

Suborder SAURIA . . . . . . . . . . . . 45

Family Gekkonidae . . . . . . . . . . . . 45

Genus Hemidactylus . . . . . . . . . . . . . . 45

Genus Phyllodactylus . . . . . . . . . . . . 45

Genus Sphaerodaetylus . . . . . . . . . . 46

Genus Coleonyx . . . . . . . . . . . . . . 47

Family Iguanidae . . . . . . . . . . . . . . . . 47

Genus Anolis . . . . . . . . . . . . . . . . . . 47

Genus Ctenosaura . . . . . . . . . . . . . . . . . 48

Genus Dipso-saurus . . . . . . . . . . . . . . . . 48

Genus Crotaphytus . . . . . . . . . . . . . . . . . 49

Genus Sauromalus . . . . . . . . . . . . 51

Genus Callisaurus . . . . . . . . . . . . . 52

Genus Uma . . . . . . . . . . . . . . . . . . 53

Genus Holbrookia . . . . . . . . . . . . . . . 53

Genus Uta . . . . . . . . . . . . . . . 55

Genus Sator . . . . . . . . . . . . . . 59

Genus Sceloporus. . . . . . . . . . . . 60

Genus Phrynosoma . . . . . . . . . . . . . . 66

Family Anguidae . . . . . . . . . . . . . . . . . 69

Genus Ophisaurus . . . . . . . . . . . . . 69

Genus Gerrhonotus . . . . . . . . . 70

Genus Barissia . . . . . . . . . . . . . . . . . 72

Family Anniellidae . . . . . . . . . . . . . . . . . 72

Genus Anniella. . . . . . . . . . . . . . . 72 
Family Helodermatidae . . . . . . . . . . . . . . . 73

Genus Heloderma . . . . . . . . . . . . . . 73

Family Xantusiidae . . . . . . . . . . . . 73

Genus Xantusia . . . . . . . . . . . 73

Family Teiidae. . . . . . . . . . . . . . . . . . 74

Genus Cnemidophorus . . . . . . . . . . . 74

Family Scincidae . . . . . . . . . . . . . . 79

Genus Leiolopisma . . . . . . . . . . . . . . . . 79

Genus Eumeces . . . . . . . . . . . . . . . . . 80

Genus Neoseps . . . . . . . . . . . . 83

Family Amphisbaenidae . . . . . . . . . . . . . 83

Genus Bipes . . . . . . . . . . . . . . . 83

Genus Rhineura . . . . . . . . . . . . 84

Suborder Serpentes . . . . . . . . . . . . . 84

Family Leptotyphlopidae . . . . . . . . . . . . . . . 84

Genus Leptotyphlops . . . . . . . . . . . . . . . 84

Family Boidae . . . . . . . . . . . . . . . . 85

Genus Lichanura . . . . . . . . . . . . . 85

Genus Charina . . . . . . . . . . . . . 86

Family Colubridae . . . . . . . . . . . . . . . . 87

Genus Carphophis . . . . . . . . . . . . . 87

Genus Abastor . . . . . . . . . . . . . . . . . 88

Genus Farancia . . . . . . . . . . . . . . . . . 88

Genus Diadophis . . . . . . . . . . . . . . . . 88

Genus Heterodon. . . . . . . . . . . . . . . . 91

Genus Liopeltis . . . . . . . . . . . . . 92

Genus Opheodrys . . . . . . . . . . . . . 93

Genus Coluber . . . . . . . . . . . . . . 93

Genus Salvadora . . . . . . . . . . . . . . 97

Genus Phyllorhynchus . . . . . . . . . . . . 97

Genus Elaphe . . . . . . . . . . . . . . 98

Genus Drymobius . . . . . . . . . . . . . . . 101

Genus Drymarchon . . . . . . . . . . . . . 101

Genus Arizona . . . . . . . . . . . . . . . . . . . 101

Genus Pituophis . . . . . . . . . . . . . . . . 102

Genus Rhadinea . . . . . . . . . . . 105

Genus Lampropeltis . . . . . . . . . . . . . . 105

Genus Stilosoma . . . . . . . . . . . 111

Genus Contia .. . . . . . . . . . . . 111

Genus Cemophora . . . . . . . . . . . . 111

Genus Rhinocheilus. . . . . . . . . . . 112

Genus Sonora . . . . . . . . . . . 112

Genus Ficimia . . . . . . . . . . . . . . . . . 113

Genus Hypsiglena . . . . . . . . . . . . . . . . . 113

Genus Chilomeniscus . . . . . . . . . . . 114

Genus Natrix . . . . . . . . . . . . . . 114

Genus Seminatrix .. . . . . . . . . . 118

Genus Storeria . . . . . . . . . . . 118 
Genus Virginia . . . . . . . . . . . 119

Genus Potamophis . . . . . . . . . . . 120

Genus Tropidoclonion. . . . . . . . . . . . . 120

Genus Liodytes . . . . . . . . . . . . . . 121

Genus Thamnophis . . . . . . . . . 121

Genus Coniophanes . . . . . . . . . . . . 126

Genus Oxybelis . . . . . . . . . . . . 127

Genus Leptodeira . . . . . . . . . . . . . . . 127

Genus Trimorphodon . . . . . . . . . . . . . 127

Genus Tantilla . . . . . . . . . . . . . . . . 128

Family Elapidae . . . . . . . . . . . . . 129

Genus Micrurus . . . . . . . . . . . . . . . . . 129

Genus Micruroides . . . . . . . . . . . 130

Family Hydrophidae . . . . . . . . . . . . 130

Genus Pelamydrus . . . . . . . . . . . 130

Family Crotalidae . . . . . . . . . . . 131

Genus Agkistrodon . . . . . . . . . . . . . 131

Genus Sistrurus . . . . . . . . . . 131

Genus Crotalus . . . . . . . . . . 132

Subclass Synapsida . . . . . . . . . . . . 137

Order Testudinata . . . . . . . . . . . . . . 137

Family Kinosternidae . . . . . . . . . . . 137

Genus Sternotherus . . . . . . . . . 137

Genus Kinosternon . . . . . . . . . . . . . . . . 138

Family Chelydridae . . . . . . . . . . . 140

Genus Macrochelys . . . . . . . . . . . 140

Genus Chelydra . . . . . . . . . . . . . . 140

Family Testudinidae . . . . . . . . . . . . 141

Genus Clemmys . . . . . . . . . . . 141

Genus Emys . . . . . . . . . . . . . 142

Genus Terrapene . . . . . . . . . . . 142

Genus Malaclemys . . . . . . . . . . . . . 143

Genus Graptemys . . . . . . . . . . . . . . 144

Genus Chrysemys . . . . . . . . . . . . . 146

Genus Pseudemys . . . . . . . . . . . . 147

Genus Deirochelys . . . . . . . . . . . . . . . . . 149

Genus Gopherus . . . . . . . . . . . . . . . 149

Family Cheloniidae . . . . . . . . . . 150

Genus Chelonia . . . . . . . . . . . . 150

Genus Eretmochelys . . . . . . . . . . . 151

Genus Caretta . . . . . . . . . . . . . 151

Family Dermochelidae . . . . . . . . . . 152

Genus Dermochelys . . . . . . . . . 152

Family Trionychidae . . . . . . . . . . . 153

Genus Amyda . . . . . . . . . . . . . 153 


\title{
AMPHIBIA (CLASS)
}

LinNé, Syst. Nat., Ed. 10, Vol. 1, 1758, p. 194

\author{
CAUDATA (ORDER) \\ Oppes, Ordn. Rept., 1811, p. 72 \\ PROTEIDA (SUBORDER)
}

Cope, Journ. Ac. Nat. Sci. Phila., Ser. 2, Vol. 6, 1866, p. 102

NECTURIDAE (FAMILY)

Necturus ( $G E N U S$ )

Rafinesque, Journ. Phys. Chim. Hist. Nat., Vol. 88, June, 1819, p. 418 TYPE: maculatus $=$ maculosus

Necturus maculosus maculosus (RAFINESQUE)

MUDPUPPY, WATERDOG

Sirena maculosa RAFINESQUE

Amer. Month. Mag. Crit. Rev., Vol. 4, No. 1, Nov. 1818, p. 41.

Necturus maculosus Rafinesque

Ann. Nat., Lexington, No. 1, March 22, 1820, p. 4.

Necturus maculatus Cope

Bull. U. S. Nat. Mus., No. 34, 1889, p. 23, fig. 3.

TYPE LOCAlity: Found in the Ohio River.

RANGE: Tributaries of the Great Lakes, the Mississippi River system, the upper Hudson River, and Lake Champlain. Rivers of North and South Carolina, Georgia, and Alabama.

Necturus maculosus lewisi BrIMLEY

Necturus maculosus lewisi BRIMLEY

Journ. Elisha Mitchell Sci. Soc., Vol. 40, Dec. 1924, p. 167.

Ty PE LOCALity: Neuse River, near Raleigh, North Carolina. RANGE: Lower course of the Neuse River, North Carolina. 


\section{Necturus punctatus (GibBes)}

Menobranchus punctatus GiBBes

Proc. Amer. Assoc. Adv. Sci., Charleston, 1850, p. 159.

Necturus punctatus Cope

Bull. U. S. Nat. Mus., No. 34, 1889, p. 27, fig. 4.

TYPE LOCALITY: Dr. Schoolbred's plantation on the South

Santee River, a few miles from its mouth, South Carolina.

RANGE: Fresh waters of North and South Carolina, especially rice-field ditches.

\section{MU'TABILIA (SUBORDER) \\ Merrem, Syst. Amph., 1820, p. 166 \\ AMPHIUMIDAE (FAMILY) \\ Amphiuma ( $G E N U S$ )}

Garden, Smith's Correspondence of Linnaeus, Vol. 1, 1821, p. 599 TYPE: means

Amphiuma means Garden

BLIND EEL, CONGO EEL, CONGO SNAKE

Amphiuma means GARDEN

Smith's Correspondence of Linnaeus, Vol. 1, 1821, p. 599 (descr. p. 333).

Amphiuma means Cope

Bull. U. S. Nat. Mus., No. 34, 1899, p. 216.

TYPE LOCALITY: Not stated, but from the context evidently either Charleston or East Florida.

RANGE: From Virginia south to Florida, west to Louisiana.

\section{Amphiuma tridactylum Cuvier}

Amphiuma tridactylum Cuvier

Mém. Mus. Hist. Nat. Paris, Vol. 14, 1827, p. 7, pl. 1, figs. 4-6, pl. 2, figs. 9-18.

TYPE LOCALITY: New Orleans, Louisiana.

RANGE: Northern Florida to southern Mississippi and Louisiana, up the Mississippi Valley at least to Missouri.

\section{CRYPTOBRANCHIDAE (FAMILY)}

Cryptobranchus ( $G E N U S$ )

Leuckart, Isis, 1821, Lit. Anz., p. 259

TYPE: gigantea $=$ alleganiensis 


\section{Cryptobranchus alleganiensis (DAUdiN)}

HELL-BENDER

Salamandra alleganiensis DAUDIN

Hist. Nat. Rept., Vol. 8, 1803, p. 231.

Cryptobranchus alleghaniensis VAN DER HoEven

Tijdschr. Nat. Geschied. Physiol., Vol. 4, 1837, p. 384.

Cryptobranchus allegheniensis COPE

Bull. U. S. Nat. Mus., No. 34, 1899, p. 38, fig. 5.

TYPE LOCALITY: Allegheny Mountains in Virginia.

RANGE: Western New York, Ohio River and tributaries, and southward to Georgia and Louisiana.

\section{SALAMANDRIDAE (FAMILY) \\ Triturus ( $G E N U S$ ) \\ Rafinesque, Anal. Nat., 1815, p. 78 \\ TY PE: cristatus}

\section{Triturus meridionalis (COPE)}

Diemyctylus miniatus, subsp. meridionalis Cope

Bull. U. S. Nat. Mus., No. 17, 1880, p. 30.

Triturus meridionalis DuNN

Bull. Mus. Comp. Zoöl., Vol. 62, 1918, p. 452.

Diemyctylus viridescens meridionalis COPE

Bull. U. S. Nat. Mus., No. 34, 1889, p. 211, fig. 54.

TyPe Locality: Matamoros, Tamaulipas, Mexico.

RANGE: Texas and Tamaulipas.

Triturus torosus (RATHKE)

GIANT NEWT, CALIFORNIA NEWT

Triton torosus RATHKe (in Eschscholtz)

Zoöl. Atlas, Pt. 5, 1883, p. 12, pl. 21, fig. 15.

Notophthalmus torosus BAIRD

Journ. Ac. Nat. Sci. Phila., Ser. 2, Vol. 1, 1849, p. 284.

Diemyctylus torosus CoPE

Bull. U. S. Nat. Mus., No. 34, 1889, p. 204, fig. 51.

TYPE LOCALITY: Surroundings of the Bay of San Francisco, California.

raNGE: California, Oregon and Washington.

Triturus viridescens viridescens (RAFINESQUE)

COMMON NEWT (AQDATIC FORM), RED EFT OR EBBET (TERRESTRIAL FORM)

Triturus viridescens RAFinesQue

Ann. Nat., Lexington, No. 1, March 22, 1820, p. 5. 
Notophthalmus viridescens BAIRD

Journ. Ac. Nat. Sci. Phila., Ser. 2, Vol. 1, 1849, p. 284.

Diemyctylus viridescens COPE

Bull. U. S. Nat. Mus., No. 34, 1889, p. 207, figs. 52-53.

Ty PE LoCALrty: Lake George, Lake Champlain.

RANGE: Eastern North America, Hudson Bay to Texas, west

Illinois, Michigan, Missouri to Oklahoma, and southeastern Kansas.

\section{Triturus viridescens symmetrica (HARLAN)}

Salamandra symmetrica HARLAN

Journ. Ac. Nat. Sci. Phila., Vol. 5, 1825, p. 157.

Notophthalmus viridescens vittatus STEJNEGER and BARBour

Check List N. Amer. Amph. Rept., Ed. 1, 1917, p. 8.

Triturus dorsalis DuNN

Bull. Mus. Comp. Zoöl., Vol. 62, 1918, p. 452.

Triturus viridescens symmetrica ScHмIDT

Copeia, No. 132, 1924, p 67.

TYPE LOCALITY: South Carolina.

RANGE: North Carolina to northern Florida; west to Louisiana.

(In the coastal plain.)

\section{AMBYSTOMIDAE $(F A M I L Y)$ \\ Ambystoma ( $G E N U S$ )}

Tschudi, Mém. Soc. Sci. Nat. Neuchâtel, 1838, p. 92 TYPE: subviolacea $=$ maculatum

\section{Ambystoma annulatum CoPE}

Amblystoma annulatum Cope

Proc. Amer. Philos. Soc., Vol. 23, 1886, p. 525.

Linguaelapsus annulatus CoPE

Bull. U. S. Nat. Mus., No. 34, 1889, p. 115, fig. 25.

TYPE LOCALITY: Unknown.

RANGE: Arkansas and Missouri.

Ambystoma cingulatum Cope

Amblystoma cingulatum Cope

Proc. Ac. Nat. Sci. Phila., 1867, p. 205.

Chondrotus cingulatus CopE

Bull. U. S. Nat. Mus., No. 34, 1889, p. 100, p. 455, fig. 116.

TYPE LOCA LITY: Grahamville, South Carolina.

RANGE: South Carolina to northern Florida and Alabama. 
Ambystoma decorticatum CoPE

Amblystoma decorticatum CoPE

Proc. Amer. Philos. Soc., Vol. 23, 1886, p. 522.

Chondrotus decorticatus COPE

Bull. U. S. Nat. Mus., No. 34, 1889, p. 107, fig. 22.

TYPE LOCALITy: Port Simpson, British Columbia.

RANGE: Coastal region of southeastern Alaska and British Columbia to the Olympic Peninsula in Washington.

Ambystoma gracile (BAIRD)

Siredon gracilis $\mathbf{B A I R D}_{\mathrm{A}}$

Pacif. R. R. Rep., Vol. 10, Williamson's Route, Pt. 4, No. 4, 1859, p. 13, pl. 44, fig. 2.

Ambystoma paroticum BAIRD

Stejneger and Barbour Check List N. Amer. Amph. Rept., Ed. 2, 1923, p. 6.

Ambystoma gracile DunN

Copeia, No. 154, May 20, 1926, p. 136.

Chondrotus paroticus Cope

Bull. U. S. Nat. Mus., No. 34, 1889, p. 105, fig. 21.

TyPE Locality: Cascade Mountains, Oregon, lat. $44^{\circ} \mathrm{N}$.

RANGE: Northern California to British Columbia, Vancouver Island.

Ambystoma jeffersonianum (GreEN)

Salamandra jeffersoniana GreEN

Contr. Maclur. Lyc., Vol. 1, No. 1, Jan. 1827, p. 4, pl. 1, fig. 1. Ambystoma jeffersoniana $\mathbf{B}_{\mathbf{A I R D}}$

Journ. Ac. Nat. Sci. Phila., Ser. 2, Vol. 1, 1849, p. 283.

Amblystoma jeffersonianum Cope

Bull. U. S. Nat. Mus., No. 34, 1889, p. 89, figs. 15-16.

Ty Pe lOCAlity: Near Jefferson College, Cannonsburg, Pennsylvania.

RANGE: Canada (to Hudson Bay and Labrador) and New England, southward to Virginia, westward to Wisconsin, Illinois, and Arkansas.

\section{Ambystoma mabeei BisHoP}

Ambystoma mabeei Bishop

Journ. Elisha Mitchell Sci. Soc., Vol. 43, No. 3-4, 1928, p. 157.

TYPE LOCALITY: Low grounds of the Black River near Dunn, North Carolina.

RANGE: Apparently a restricted area about the type locality. 


\section{Ambystoma macrodactylum BAIRD}

Ambystoma macrodactyla BAIRD

Journ. Ae. Nat. Sci. Phila., Ser. 2, Vol. 1, 1849, p. 292.

Amblystoma macrodactylum CopE

Bull. U. S. Nat. Mus., No. 34, 1889, p. 95, fig. 17.

Ambystoma epixanthum STEJNEgER and Barbour

Check List N. Amer. Amph. Rept., Ed. 1, 1917, p. 9.

TYPE LOCALITY: Astoria, Oregon.

RANGE: Northern California to British Columbia and Alberta,

Idaho and Montana, probably eastward to and certainly in

Iowa.

Ambystoma maculatum (SHAw)

SPOTTED SALAMANDER

Lacerta maculata SHAw

Gen. Zool., Vol. 3, Pt. 1, 1802, p. 304.

Ambystoma maculatum STEJNeGer

Proc. Biol. Soc. Washington, Vol. 15, Dec. 16, 1902, p. 239.

Amblystoma punctatum COPE

Bull. U. S. Nat. Mus., No. 34, 1889, p. 56, fig. 9.

TYPE LOCALITY: Carolina (based on Catesby, Nat. Hist. Caro-

lina, Ed. 1, Appendix, 1748, pl. 10, fig. 10).

RANGE: Nova Scotia west to Wisconsin, southward to Georgia

(and possibly from Florida) and Texas.

Ambystoma opacum (Gravenhorst)

MARBLED SALAMANDER

Salamandra opaca Gravenhorst

Vergl. Uebers. Zool. Syst., 1807, p. 431.

Ambystoma opaca $\mathrm{B}_{\mathrm{AlRD}}$

Journ. Ac. Nat. Sci. Phila., Ser. 2, Vol. 1, 1849, p. 283.

Amblystoma opacum Cope

Buli. U. S. Nat. Mus., No. 34, 1889, p. 54, fig. 8.

TYPE LOCALITY: New York.

RANGE: From Massachusetts to Georgia, west to Louisiana and

Texas, Mississippi basin north to Arkansas, Missouri, Indiana, and Illinois.

\section{Ambystoma talpoideum (HoLBRooK)}

MOLE SALAMANDER

Salamandra talpoidea Holbrook

N. Amer. Herp., Ed. 1, Vol. 3, 1838, p. 117, pl. 29.

Ambystoma talpoideum Gray

Cat. Batr. Grad. Brit. Mus., 1850, p. 36. 
Amblystoma talpoideum Cope

Bull. U. S. Nat. Mus., No. 34, 1889, p. 52, fig. 7.

TYPE LOCALITY: Sea Islands on the border of South Carolina. RANGE: South Atlantic and Gulf States, North Carolina to Louisiana and northward to Illinois.

\section{Ambystoma texanum (MatThes)}

Salamandra texana Matthes

Allg. deutsche naturh. Zeitschr., N. S., Vol. 1, 1855, p. 266.

Amblystoma texanum Bard

U. S. Mex. Bound. Surv., Vol. 2, 1859, Rept., p. 29, pl. 35, fig. 15.

Ambystoma microstomum STEJNEGER and Barbour

Check List N. Amer. Amph. Rept., Ed. 2, 1923, p. 6.

Chondrotus texanus Cope

Bull. U. S. Nat. Mus., No. 34, 1889, p. 104, fig. 20.

TYPE LOCA LITY: "Das erste Exemplar fand ich . . . im Urwalde am Rio Colorado, das zweite ebenfalls . . . im Cumming's Creeck [sic] Bottom, Fayette County."

RANGE: Ohio to Kansas and Nebraska, east to Illinois and south to North Carolina, Tennessee, Mississippi, Louisiana and Texas.

\section{Ambystoma tigrinum (GreEN)}

TIGER SALAMANDER

Salamandra tigrina Green

Journ. Ac. Nat. Sci. Phila., Vol. 5, 1825, p. 116.

Ambystoma tigrina BAIRD

Journ. Ac. Nat. Sci. Phila., Ser. 2, Vol. 1, 1849, p. 284.

Amblystoma tigrinum CoPE

Bull. U. S. Nat. Mus., No. 34, 1889, p. 68, figs. 12-13.

ty PE LOCAlity: Near Moorestown, New Jersey.

RANGE: Widely distributed over almost all the United States, east of the Cascade Range and Sierra Nevada, and extending southward over the northern portion of the Mexican plateau.

\section{Dicamptodon ( $G E N U S$ )}

Strauch, Mém. Acad. Sci. St. Pétersb., Ser. 7, Vol. 16, No. 4, 1870, p. 68 TYPE: ensatus

\section{Dicamptodon ensatus (Eschscholtz)}

Triton ensatus EschscholTz

Zool. Atlas, Pt. 5, 1833, p. 6, pl. 22.

Dicamptodon ensatus STrauch

Mém. Ac. Sci. St. Pétersb., Ser. 7, Vol. 16, No. 4, 1870, p. 69. 
Ambystoma tenebrosum Stejneger and Barbour

Check List N. Amer. Amph. Rept., Ed. 1, 1917, p. 11.

Dicamptodon aterrimus StEJNEger and BARBour

Check List N. Amer. Amph. Rept., Ed. 2, 1923, p. 7.

TYPE LOCALity: California, environs of Bay of San Francisco.

RANGE: Central California to southern coastal region of British

Columbia, east of Sierras to Pullman, Washington.

\section{Rhyacotriton ( $G E N U S$ )}

Dunn, Proc. N. Engl. Zoöl. Club, Vol. 7, 1920, p. 56

TYPE: olympicus

\section{Rhyacotriton olympicus (GAIGE)}

Ranodon olympicus GAIGE

Occ. Papers Mus. Zool. Univ. Michigan, No. 40, May 30, 1917, p. 2, pl. 1.

Rhyacotriton olympicus DunN

Proc. N. Engl. Zoöl. Club, Vol. 7, 1920, p. 56.

Ranodon olympicus StejNeger and Barbour

Check List N. Amer. Amph. Rept., Ed. 1, 1917, p. 13.

TYPE LOCALITY: Lake Cushman, Washington.

RANGE: Western Washington south to Oregon in the mountains.

\section{PLETHODONTIDAE (FAMILY)}

\section{Batrachoseps ( $G E N U S$ )}

Bonaparte, Iconogr. Fauna Italica, Vol. 2, Fasc. 26, 1839,

fol. $131 * * *$ (not paged)

TYPE: attenuatus

\section{Batrachoseps attenuatus attenuatus (EschscholTz)}

Salamandrina attenuata EschscholTz

Zool. Atlas, Pt. 5, 1833, p. 1, pl. 21, figs. 1-14.

Batrachoseps attenuatus CoOPER

Pacif. R. R. Surv., Vol. 12, Pt. 3, 1858, Zoöl., p. viii, pl. 31, fig. 5.

Batrachoseps attenuatus Cope

Bull. U. S. Nat. Mus., No. 34, 1889, p. 127, fig. 28.

Batrachoseps attenuatus attenuatus BerRY CAMPBELL

Copeia, 1931, No. 3, Oct. 30, p. 133.

ty

RANGE: Southwestern Oregon, California, to Los Angeles County;

Pacific Border Province, and Sierra Nevada. 


\section{Batrachoseps attenuatus caudatus COPE}

Batrachoseps caudatus COPE

Bull. U. S. Nat. Mus., No. 34, 1889, p. 126, fig. 27.

Batrachoseps attenuatus caudatus BERRY CAMPBELL

Copeia, 1931, No. 3, Oct. 30, p. 133.

TyPe locality: Hassler Harbor, Alaska. (Probably on Anette Island, S. E. Alaska.)

RANGE: Known only from type locality and doubtfully from Yukatat Bay.

\section{Batrachoseps attenuatus leucopus (DUNN)}

Batrachoseps leucopus DunN

Copeia, No. 109, Aug. 15, 1922, p. 60.

Batrachoseps attenuatus leucopus BERRY CAMPBELL

Copeia, 1931, No. 3, Oct. 30, p. 133

TYPE LOCALITY: North Island, Los Coronados Islands.

RANGE: Los Coronados Islands; Lower California, and adjacent coast of California.

\section{Batrachoseps pacificus pacificus (COPE)}

Hemidactylium pacificum Cope

Proc. Ac. Nat. Sci. Phila., 1865, p. 195.

Batrachoseps pacificus CoPE

Proc. Ac. Nat. Sci. Phila., 1869, p. 99.

Batrachoseps pacificus CoPE

Bull. U. S. Nat. Mus., No. 34, 1889, p. 129.

Batrachoseps pacificus pacificus BERRY CAMPBELL

Copeia, 1931, No. 3, Oct. 30, p. 133.

TyPE LOCALITy: Santa Barbara (?), California.

RANGE: San Miguel, Santa Rosa and Santa Cruz Islands off the coast of California.

\section{Batrachoseps pacificus major (CAMP)}

Batrachoseps major CAMP

Univ. California Publ. Zool., Vol. 12, No. 12, Apr. 2, 1915, p. 327.

Batrachoseps pacificus major BERRY CAMPBELL

Copeia, 1931, No. 3, Oct. 30, p. 133.

TYPE LOCAlity: Sierra Madre, 1000 feet elevation, Los Angeles Co., California.

RANGE: Los Angeles, Riverside and Orange Counties, southwestern California. 


\section{Batrachoseps pacificus catalinae (DUNN)}

Batrachoseps catalinae DUNN

Copeia, No. 109, Aug. 15, 1922, p. 62.

Batrachoseps pacificus catalinae BerRy CAMPBELL

Copeia, 1931, No. 3, Oct. 30, p. 133.

TYPE LOCALITY: Santa Catalina Island.

RANGE: Same.

\section{Hemidactylium ( $G E N U S$ )}

Tschudi, Mém. Soc. Sci. Nat. Neuchâtel, 1838, p. 94 TYPE: scutatum

\section{Hemidactylium scutatum (SCHLEGEL)}

FOUR-TOED SALAMANDER

Salamandra scutata SCHLEgeL

Fauna Japonica, Saur. Batr., 1838, p. 119.

Hemidactylium scutatum TSCHUDI

Mém. Soc. Sci. Nat. Neuchâtel, 1838, p. 94.

Hemidactylium scutatum Cope

Bull. U. S. Nat. Mus., No. 34, 1889, p. 130, fig. 29.

Ty PE LOCALity: Nashville, Tennessee.

RANGE: Massachusetts, southern Canada westward to Michigan,

south to Georgia and the Gulf States, also Arkansas and Illinois.

\section{Plethodon ( $G E N U S)$}

Tschudi, Mém. Soc. Sci. Nat. Neuchâtel, 1838, p. 92 TYPE: glutinosus

\section{Plethodon cinereus (GREEN)}

REDBACKED SALAMANDER, DUSKY SALAMANDER (NAMES FOR THE TWO COLOR PHASES)

Salamandra cinerea Green

Journ. Ac. Nat. Sci. Phila., Vol. 1, Pt. 2, Sept. 1818, p. 356.

Plethodon cinereus Tschudi

Mém. Soc. Sci. Nat. Neuchâtel, 1838, p. 92.

Plethodon cinereus CoPE

Bull. U. S. Nat. Mus., No. 34, 1889, p. 133, fig. 30.

TYPE LOCALITY: New Jersey.

RANGE: Cape Breton Island west to Fort William Ontario, south

to Dallas, Georgia, Missouri and Arkansas. 
Plethodon clemsonae BRIMLEY ${ }^{1}$

Plethodon clemsonae ${ }^{1}$ Brinlex

Copeia, No. 164, July-Sept. 1927, p. 73.

TYPE LOCALITY: Jocassee, S. C.

RANGE: Same.

\section{Plethodon dorsalis (CoPE)}

Plethodon cinereus dorsalis Cope

Bull. U. S. Nat. Mus., No. 34, 1889, p. 138.

Plethodon dorsalis StEJNEGER and Barbour

Check List N. Amer. Amph. Rept., Ed. 1, 1917, p. 15.

TYPE LOCALity: Salem, Massachusetts (?), and Louisville, Kentucky.

RA NG E: Western Pennsylvania, southern Ohio, southern Indiana, central Kentucky, Tennessee, and Alabama.

\section{Plethodon elongatus Van Denburgi}

Plethodon elongatus VAN DENBURGH

Proc. Calif. Ac. Sci., Ser. 4, Vol. 6, No. 7, May 12, 1916, p. 216.

TYPE locality: Requa, Del Norte Co., California.

RANGE: Requa, Del Norte Co., California.

\section{Plethodon glutinosus (GreEN)}

\section{SLIMY SALAMANDER}

Salamandra glutinosa Green

Journ. Ac. Nat. Sci. Phila., Vol. 1, Pt. 2, Sept. 1818, p. 357.

Plethodon glutinosus TschUd

Mém. Soc. Sci. Nat. Neuchâtel, 1838, p. 92.

Plethodon glutinosus CoPE

Bull. U. S. Nat. Mus., No. 34, 1889, p. 139, fig. 31.

TyPe LOCALity: Not mentioned but obviously Princeton, New Jersey. Here Green lived, and he mentions often finding the creature under stones.

RANGE: Southern New York to Wisconsin, south to northern Florida, the Gulf States to Texas and to Missouri.

\section{Plethodon intermedius BAIRD}

Plethodon intermedius BAIRD

Proc. Ac. Nat. Sci. Phila., 1867, p. 209.

Plethodon intermedius Cope

Bull. U. S. Nat. Mus., No. 34, 1889, p. 145, fig. 33.

It has been suggested that this is $P$. metcalf, and this is probably a correct view. 
TYPE LOCALITY: Fort Tejon, California (?).

RANGE: Vancouver Island; Fraser River, British Columbia south to Salem, Oregon.

\section{Plethodon jordani Blatchley}

Plethodon jordani Blatchlex

25 Ann. Rep. Dept. Geol. Indiana, 1900 (1901), p. 762.

TyPe locality: Mt. Collins and Indian Pass, 3000-5000 feet altitude, Sevier Co., Tenn.

R A NGE: Tennessee and North Carolina (Great Smoky Mountains).

\section{Plethodon metcalfi BRIMLEY}

Plethodon metcalfi BRIMLEY

Proe. Biol. Soc. Washington, Vol. 25, Dec. 4, 1912, p. 138, pl. 6, figs. $1,2,4,7-11$.

TYPE LOCALITY: Sunburst, Haywood Co., North Carolina.

RANGE: Southwestern Virginia south through western North Carolina, northern Georgia and northeastern Alabama. It apparently occupies all the ranges of the Southern Blue Ridge except the Great Smokies and the Nantahalas.

\section{Plethodon shermani Stejneger}

Plethodon shermani STEJNEGER

Proc. U. S. Nat. Mus., Vol. 30, May 22, 1906, p. 559, fig. 1.

TyPE LOCALITy: ${ }^{1}$ Wayah Bald Mountain, North Carolina.

RANGE: Southwestern North Carolina (Nantahala Mountains).

\section{Plethodon vandykei VAN Denburgh}

Plethodon vandykei $\mathrm{V}_{\text {an }}$ Denburgh

Proc. Calif. Ac. Sci., Ser. 3, Zool., Vol. 4, March 14, 1906, p. 61.

ty ton.

RANGE: Western Washington (Cascade and Olympic Mountains).

\section{Plethodon wehrlei Fowler and DUNN}

Plethodon wehrlei Fowler and DUNN

Proc. Ac. Nat. Sci. Phila., Vol. 69, 1917, p. 23, pl. 4.

ty Pe LOCALIty: Two Lick Hills, Indiana Co., Pennsylvania. RANGe: Western New York, Pennsylvania and West Virginia.

Northern section of Appalachian Plateau.

\footnotetext{
1 Originally given as Nantahala Mt. between Andrews and Aquone, North Carolina (in errore),
} Wayah Bald is, however, in the Nantahala range, but is not between Andrews and Aquone. 
Plethodon welleri WaLkeR

Plethodon welleri WaLKer

Proc. Junior Soc. Nat. Sci., Cincinnati, Vol. 2, July 31, 1931, p. 48.

TYPE LOCALITY: Grandfather Mt., above $5000 \mathrm{ft}$., near Linville,

North Carolina.

RANGE: The type locality.

\section{Plethodon yonahlossee DUNN}

Plethodon yonahlossee DunN

Bull. Amer. Mus. Nat. Hist., Vol. 37, Oct. 13, 1917, p. 598.

TYPE LOCALity: Near the Yonahlossee Road, about $1 \frac{1}{2}$ miles

from Linville, North Carolina, altitude 4200 feet.

RANGE: Wooded mountains near Linville, North Carolina, and in southwestern Virginia.

\section{Ensatina ( $G E N U S)$}

GraY, Cat. Batr. Grad. Brit. Mus., 1850, p. 48 TYPE: eschscholtzii

Ensatina croceater (COPE)

Plethodon croceater $\mathrm{COPE}$

Proc. Ac. Nat. Sci. Phila., 1867, p. 210.

Ensatina croceater DunN

Proc. N. Engl. Zool. Club, Vol. 8, Feb. 27, 1923, p. 39.

Plethodon croceater CoPE

Bull. U. S. Nat. Mus., No. 34, 1889, p. 150.

Plethodon croceater STEJNeger and Barbour

Check List N. Amer. Amph. Rept., Ed. 1, 1917, p. 14.

TYPE LOCALITY: Fort Tejon, California (?).

RANGE: Eastern California from Placer County to Kern County, in the Sierra Nevada. Lower California (?).

\section{Ensatina eschscholtzii GraY}

Ensatina eschscholtzii GraY

Cat. Batr. Grad. Brit. Mus., 1850, p. 48.

Plethodon oregonensis CopE

Bull. U. S. Nat. Mus., No. 34, 1889, p. 148, fig. 35.

Plethodon eschscholtzii STEJNEGER and BARBOUR

Check List N. Amer. Amph. Rept., Ed. 1, 1917, p. 15.

TYPE LOCALITY: California.

RANGE: Puget Sound to Lower California (Cascade, Olympic, San

Bernardino and San Pedro Martir Mountains). 
Ensatina sierrae STORER

Ensatina sierrae STORER

Univ. of Calif. Publ. Zool., Vol. 30, No. 16, June, 1929, p. 448.

Ty P LoCAlity: Yosemite Valley, 7300 feet altitude, Mariposa Co., California.

RANGE: Transition Zone of the Sierra Nevada Mountains from

Tulare to Eldorado Counties, California.

Typhlotriton ( $G E N U S$ )

Stejneger, Proc. U. S. Nat. Mus., Vol. 15, 1892, p. 115 TYPE: spelaeus

Typhlotriton spelaeus STEJNEGER

Typhlotriton spelaeus STEJNeger

Proc. U. S. Nat. Mus., Vol. 15, Aug. 2, 1892, p. 115, pl. 9.

TyPE LOCALity: Rock House Cave, Missouri.

RANGE: Ozark Plateau in Missouri, Kansas and Arkansas.

\section{Stereochilus ( $G E N U S$ )}

Cope, Proc. Ac. Nat. Sci. Phila., 1869, p. 100

TYPE: marginatus

\section{Stereochilus marginatus (HALLOWELL)}

Pseudotriton marginatus HALlowell

Proc. Ac. Nat. Sci. Phila., 1856, p. 130.

Stereochilus marginatum CopE

Proc. Ac. Nat. Sci. Phila., 1869, p. 101.

Stereochilus marginatus CoPE

Bull. U. S. Nat. Mus., No. 34, 1889, p. 152, fig. 36.

TYPE LOCALITY: Liberty County, Georgia.

RANGE: Dismal Swamp Virginia to Liberty County, Georgia, in the Coastal Plain.

\section{Typhlomolge ( $G E N U S$ )}

Stejneger, Proc. U. S. Nat. Mus., Vol. 18, 1896, p. 620 TYPE: rathbuni

Typhlomolge rathbuni STEJNEgER

Typhlomolge rathbuni STEJNEGER

Proc. U. S. Nat. Mus., Vol. 18, Apr. 15, 1896, p. 620. 
TyPe LOCAlity: Artesian well 188 feet deep, at U. S. Fish Commission Station, San Marcos, Hays Co., Texas.

RANGE: Known from the vicinity of the type locality only.

\section{Gyrinophilus ( $G E N U S$ )}

Cope, Proc. Ac. Nat. Sci. Phila., 1869, p. 108

TYPE: porphyriticus

Gyrinophilus porphyriticus porphyriticus (GREEN)

Salamandra porphyritica GREEN

Contr. Maclur. Lyc., Vol. 1, 1827, p. 3, pl. 1, fig. 2.

Gyrinophilus porphyriticus COPE

Proc. Ac. Nat. Sci. Phila., 1869, p. 108.

Gyrinophilus porphyriticus COPE

Bull. U. S. Nat. Mus., No. 34, 1889, p. 155, fig. 37.

TyPE LOCALity: French Creek near Meadville, Crawford Co., Pennsylvania.

RANGE: Maine to Ontario, Canada southward through the eastern states to Virginia, Ohio, Tennessee and northern Alabama (Appalachian uplift except southern Blue Ridge; Adirondacks; and the eastern edge of the central Low Plateau).

Gyrinophilus porphyriticus danielsi (BLATCHLEY)

Spelerpes danielsi BLATCHLEY

25 Ann. Rep. Dept. Geol. Indiana, 1900 (1901), p. 760.

Gyrinophilus danielsi FowLer and DUNN

Proc. Ac. Nat. Sci. Phila., Vol. 69, 1917, p. 19.

ty pe locality: Mt. Collins and Indian Pass, Sevier Co., Tennessee.

RANGE: Southern section of the Blue Ridge Mountains in western North Carolina and eastern Tennessee. Perhaps South Carolina and Georgia.

\section{Gyrinophilus porphyriticus duryi (WALLER)}

Pseudotriton duryi WALLER

Proc. Junior Soc. Nat. Sci., Cincinnati, Vol. 1, 1930, Nos. 5-6, p. [6]. TYPE localitx: Cascade Caves, near Grayson, Carter Co., Kentucky.

RANGE: Northeastern Kentucky and southern Ohio.

Manculus ( $G E N U S$ )

Cope, Proc. Ac. Nat. Sci. Phila., 1869, p. 101

TY PE: quadridigitatus 
Manculus quadridigitatus quadridigitatus (HOLBROOK) DWARF SALAMANDER

Salamandra quadridigitata HoLBRoок

N. Amer. Herp. Ed. 2, Vol. 5, 1842, p. 65, pl. 21.

Manculus quadridigitatus CopE

Proc. Ac. Nat. Sci. Phila., 1869, p. 101.

Manculus quadridigitatus COPE

Bull. U. S. Nat. Mus., No. 34, 1889, p. 159, fig. 38.

TYPE LOCALity: Georgia, South Carolina, and Florida.

RANGE: North Carolina south to southern Georgia and westward through the Gulf States to Texas.

\section{Manculus quadridigitatus remifer (COPE)}

Manculus remifer CoPE

Rep. Peabody Ac. Salem, 1869-1870 (1871), p. 84.

Manculus remifer Cope

Bull. U. S. Nat. Mus., No. 34, 1889, p. 158.

TYPE LOCALITY: Jacksonville, Florida.

RANGE: East Florida south to Sebastian.

\section{Pseudotriton ( $G E N U S$ )}

Tschudi, Mém. Soc. Sci. Nat. Neuchâtel, 1838, p. 60 TYPE: subfuscus $=$ ruber

\section{Pseudotriton montanus montanus (BAIRD)}

Pseudotriton montanus BAIRD

Journ. Ac. Nat. Sci. Phila., Ser. 2, Vol. 1, 1849, p. 293.

Spelerpes ruber montanus COPE

Bull. U. S. Nat. Mus., No. 34, 1889, p. 179.

Eurycea montana STEJNEger and BARBour

Check List N. Amer. Amph. Rept., Ed. 1, 1917, p. 19.

ty Pe locality: South Mountain, near Carlisle, Penn.

RANGe: New York, Pennsylvania, southern Ohio to western

Georgia, Kentucky, and Tennessee.

\section{Pseudotriton montanus flavissimus (HALLOWELL)}

Pseudotriton flavissimus Hallowell

Proc. Ac. Nat. Sci. Phila., 1856, p. 130.

TYPE LOCALITY: Liberty County, Georgia.

RANGE: Coastal plain of Georgia to eastern Louisiana. 


\section{Pseudotriton ruber ruber (SoNNINI)}

RED SALAMANDER

Salamandra rubra SoNNiNI

in Latreille, Hist. Nat. Rept., Vol. 4, 1802, p. 305.

Pseudotriton ruber ruber DUNN

Proc. Biol. Soc. Washington, Vol. 33, Dec. 30, 1920, p. 133.

Spelerpes ruber CoPE

Bull. U. S. Nat. Mus., No. 34, 1889, p. 172, fig. 43.

Eurycea rubra rubra StejNeger and BARBour

Check List N. Amer. Amph. Rept., Ed. 1, 1917, p. 20.

TYPE LOCALITY: United States.

RANGE: From Albany County, New York to northern Florida westward to Ohio, Kentucky, Tennessee, Alabama and northern Mississippi.

\section{Pseudotriton ruber nitidus DunN}

Pseudotriton ruber nitidus DunN

Proc. Biol. Soc. Washington, Vol. 33, Dec. 30, 1920, p. 133.

Ty PE LoCAli Ty: White Top Mt., Virginia, 4000 feet.

RANGE: Area in southwestern Virginia, northwestern North Caro-

lina and northeastern Tennessee bounded by Stone and Iron

Mts. to the west, the Blue Ridge to the east, the Black Mts.

to the south; northern plateau of southern Blue Ridge.

\section{Pseudotriton ruber schencki (BRIMLEY)}

Spelerpes ruber schencki BrinLeY

Proc. Biol. Soc. Washington, Vol. 25, Dec. 4, 1912, p. 139, pl. 7, figs. $12,13,15,17,18,20-22$.

Eurycea rubra schencki STEJNEger and BARBour

Check List N. Amer. Amph. Rept., Ed. 1, 1917, p. 20.

TYPE LOCALITY: Sunburst, North Carolina, 3200 feet.

RANGE: Southern Blue Ridge Mts. in southwestern North Caro-

lina and northern Georgia. Reaches 3500 feet in the Balsam Mts.

\section{Eurycea ( $G E N U S$ )}

Rafinesque, Kentucky Gazette, Lexington, N. S., Vol. 1, No. 9, Feb. 28, 1822 , p. 3 , column 6

TYPE: lucifuga

\section{Eurycea bislineata bislineata (GREEN)}

Salamandra bislineata Green

Journ. Ac. Nat. Sci. Phila., Vol. 1, Sept. 1818, p. 352. 
Eurycea bislineata bislineata DuNN

Proc. Biol. Soc. Washington, Vol. 33, Dec. 30, 1920, p. 134.

Spelerpes bilineatus COPE

Bull. U. S. Nat. Mus., No. 34, 1889, p. 163, fig. 40.

TYPE LOCALity: Western Pennsylvania.

RANGE: Quebec, New Brunswick, to Indiana, Tennessee, and Virginia.

Eurycea bislineata cirrigera (GREEN)

Spelerpes cirrigera Green

Journ. Ac. Nat. Sci. Phila., Vol. 6, 1839, p. 253.

Eurycea bislineata cirrigera DuNN

Proc. Biol. Soc. Washington, Vol. 33, Dec. 30, 1920, p. 135.

TY PE LOCAL I TY: New Orleans, Louisiana.

RANGE: North Carolina south through northwestern Florida, west through Alabama and southern Louisiana; western Tennessee.

\section{Eurycea bislineata wilderae DuNN}

Eurycea bislineata wilderae DuNN

Proc. Biol. Soc. Washington, Vol. 33, Dec. 30, 1920, p. 134.

Ty PE LoCAlity: White Top Mt., Virginia, 4000 feet.

RANGE: The Southern Blue Ridge region from White Top Mountain, Virginia south through North Carolina, South Carolina, and Tennessee to Rabun and Gilmer Counties, Georgia.

\section{Eurycea gutto-lineata (НоLBRoок)}

Salamandra gutto-lineata HoLBRook

N. Amer. Herp., Ed. 1, Vol. 2, 1838, p. 61, pl. 12.

Eurycea gutto-lineata STEJNEGER and BARBour

Check List N. Amer. Amph. Rept., Ed. 1, 1917, p. 19.

Spelerpes guttolineatus CoPE

Bull. U. S. Nat. Mus., No. 34, 1889, p. 170, fig. 42.

TYPE LOCALITY: "Carolina in the middle country."

RANGE: Fairfax County, Virginia to Liberty County, Georgia, west to Alabama, Mississippi, and Louisiana. Western Tennessee. Region includes southern Coastal Plain and Piedmont, southern Blue Ridge, and extreme southern part of the Appalachian Valley.

\section{Eurycea longicauda (GREeN)}

Salamandra longicauda Green

Journ. Ac. Nat. Sci. Phila., Vol. 1, Sept. 1818, p. 351. 
Eurycea longicauda Stejneger and BARBour

Check List N. Amer. Amph. Rept., Ed. 1, 1917, p. 19.

Spclcrpes longicaudus CoPE

Bull. U. S. Nat. Mus., No. 34, 1889, p. 168, fig. 41.

TYPE LOCALITY: New Jersey (probably near Princeton).

RANGE: Central New York to northern Georgia, westward to southern Missouri and northeastern Arkansas.

\section{Eurycea lucifuga Rafinesque}

Eurycea lucifuga RAFINESQUE

Kentucky Gazette, Lexington, N. S., Vol. 1, No. 9, Feb. 28, 1822, p. 3 , column 6 .

Gyrinophilus maculicaudus Cope

Amer. Nat., Vol. 24, 1890, p. 967.

TYPE LOCALITY: "Caves near Lexington, Kentucky."

RANGE: West Virginia, Tennessee, and northern Alabama westward through southern Ohio, Kentucky, and southern Indiana to Missouri and northwestern Arkansas.

\section{Eurycea melanopleura (COPE)}

Spelerpes melanopleurus CopE

Proc. Ac. Nat. Sci. Phila., 1893, p. 383.

Eurycea melanopleura STEJNEGER and BARBour

Check List N. Amer. Amph. Rept., Ed. 1, 1917, p. 19.

Ty PE LOCAlity: Raley's Creek, White River, Missouri.

RANGe: Franklin and Jasper Counties, Missouri to Pulaski

County, Arkansas. Extends into Texas and probably into

Oklahoma and Kansas.

\section{Eurycea multiplicata (CoPE)}

Spelerpes multiplicatus CoPE

Proc. Ac. Nat. Sci. Phila., 1869, p. 106.

Eurycea multiplicata STEJNEGER and BARBOUR

Check List N. Amer. Amph. Rept., Ed. 1, 1917, p. 20.

Spelerpes multiplicatus CoPe

Bull. U. S. Nat. Mus., No. 34, 1889, p. 162, fig. 39.

TYPE LOCALITY: Red River, in eastern Oklahoma.

RANGE: Stone County, Missouri to Pulaski County, Arkansas; the Jemez Mountains, New Mexico. Distribution apparently discontinuous. 


\section{Hydromantes ( $G E N U S$ )}

Gistel, Naturg. 'Thierr., 1848, p. xi TYPE: genei

\section{Hydromantes platycephala (CAMP)}

Spelerpes platycephalus CAMP

Univ. Calif. Publ. Zool., Vol. 17, Sept. 18, 1916, p. 11.

Iydromantes platycephalus DunN

Proc. N. Engl. Zoöl. Club. Vol. 8, Feb. 27, 1923, p. 40.

Eurycea platycephala STEJNEGER and BARBOUR

Check List N. Amer. Amph. Rept., Ed. 1, 1917, p. 20.

TYPE LOCALITY: Head of Lyell Cañon, the Yosemite National

Park, California, 10,800 feet alt.

RANGE: Head of Lyell Cañon, the Yosemite National Park, Califormia, 10,800 feet alt.

\section{Aneides ( $G E N U S$ )}

BAIRD, Iconogr. Encycl., Vol. 2, 1849, Zoöl., p. 257 TYPE: lugubris

\section{Aneides aeneus (Cope)}

Plethodon aeneus COPE

Amer. Nat., Vol. 15, 1881, p. 878.

Aneides aeneus DunN

Proc. N. Engl. Zoöl. Club, Vol. 8, Feb. 27, 1923, p. 39.

Plethodon aeneus Stejneger and Barbour

Check List N. Amer. Amph. Rept., Ed. 1, 1917, p. 14.

Plethodon aeneus Cope

Bull. U. S. Nat. Mus., No. 34, 1889, p. 143, fig. 32.

TYPE LOCALITY: Nickajack Cave, southern Tennessee.

RANGE: West Virginia to Tennessee, in the Cumberland Mountains.

\section{Aneides ferreus Cope}

Anaides ferreus Cope

Proc. Ac. Nat. Sci. Phila., 1869, p. 109.

Autodax ferreus Cope

Bull. U. S. Nat. Mus., No. 34, 1889, p. 185, fig. 45.

TYPE LOCALITY: Fort Umpqua, Oregon.

RANGE: Vancouver Island, British Columbia to Mendocino

County, California. Northern part of Pacific Border Province. 


\section{Aneides flavipunctatus (STRAUCH)}

Plethodon flavipunctatus STrauch

Mém. Ac. Sci. St. Pétersb., Ser. 7, Vol. 16, No. 4, 1870, p. 71.

Aneides flavipunctatus STORER

Univ. Calif. Publ. Zool., No. 27, 1925, p. 119.

Aneides iëcanus S'TEJNEger and BARBour

Check List N. Amer. Amph. Rept., Ed. 2, 1923, p. 18.

TyPE lOCAlity: New Albion, California [probably in Sonoma

Co. fide Storer].

RANGE: Northern California, Coast, Klamath and Southern Cascade Ranges.

Aneides lugubris lugubris (HaLlowell)

Salamandra lugubris Halloweld

Proc. Ac. Nat. Sci. Phila., Vol. 4, 1849, p. 126.

Aneides lugubris BAIRD

Iconogr. Encycl., Vol. 2, 1849, Zoöl., p. 257.

Autodax lugubris Cope

Bull. U. S. Nat. Mus., No. 34, 1889, p. 183, fig. 44.

TYPE LOCALITY: Monterey, California.

RANGE: California; Coronados Islands, Lower California.

Aneides lugubris farallonensis (Van Denburgh)

Autodax lugubris farallonensis VAN DENBURGH

Proc. Calif. Ac. Sci., Ser. 3, Zool., Vol. 4, 1905, p. 5, pl. 2.

Aneides lugubris farallonensis Grinnell and CAMP

Univ. California Publ. Zool., Vol. 17, July 11, 1917, p. 135.

TYPE LOCALITY: South Farallon Island, California.

RANGE: The Farallon Islands.

\section{Desmognathus ( $G E N U S$ )}

BAird, Journ. Ac. Nat. Sci. Phila., Ser. 2, Vol. 1, 1849, pp. 282, 285 TYPE: fuscus

\section{Desmognathus brimleyorum STEJNEGER}

Desmognathus brimleyorum STEJNEGer

Proc. U.S. Nat. Mus., Vol. 17, May 11, 1895, p. 597.

ty

RANGE: Arkansas, Texas, and Oklahoma.

Desmognathus fuscus fuscus (RAfinesque)

Triturus fuscus RAFINESQUE

Ann. Nat., Lexington, No. 1, March 22, 1920, p. 4. 
Desmognathus fuscus BAIRD

Journ. Ac. Nat. Sci. Phila., Ser. 2, Vol. 1, 1849, p. 285.

Desmognathus fusca fusca COPE

Bull. U. S. Nat. Mus., No. 34, 1889, p. 194, fig. 48.

TYPE LOCALITY: Northern parts of State of New York.

RANGE: St. John's River, New Brunswick to northwestern

Florida, Mississippi, and Illinois. Manitoba?

\section{Desmognathus fuscus auriculatus (НоцвRоок)}

Salamandra auriculata HoLBrook

N. Amer. Herp., Ed. 1, Vol. 3, 1838, p. 115, pl. 28.

Desmognathus fusca auriculata COPE

Bull. U. S. Nat. Mus., No. 34, 1889, p. 195, fig. 49.

Desmognathus auriculatus BA1RD

Journ. Ac. Nat. Sci. Phila., Ser. 2, Vol. 1, 1849, p. 286.

TYPE LOCALITY: Riceborough, Georgia.

RANGE: Lowlands from Virginia to Florida, west to Mississippi in the coastal plain.

\section{Desmognathus ochrophaeus ochrophaeus (COPE)}

Desmognathus ochrophaea CoPE

Proc. Ac. Nat. Sci. Phila., 1859, p. 124.

Desmognathus ochrophaea CoPE

Bull. U. S. Nat. Mus., No. 34, 1889, p. 191, fig. 47.

TyPE LOCAlity: Susquehanna County, Pennsylvania.

RANGE: Clinton County, New York to Garrett County, Maryland, west to Columbus, Ohio, in the Allegheny Plateau and the Appalachian Valley.

\section{Desmognathus ochrophaeus carolinensis DunN}

Desmognathus ochrophaea carolinensis DuNN

Proc. Biol. Soc. Washington, Vol. 29, April 4, 1916, p. 74.

ty PE LOCALity: Mt. Mitchell, North Carolina, over 6500 feet. RANGe: Beverly, West Virginia to Gwinnett County, Georgia (in the mountains).

\section{Desmognathus phoca (Matthes)}

Salamandra phoca Mattues

Allg. deutsche naturh. Zeitschr., N. S., Vol. 1, 1855, p. 273.

Desmognathus phoca DuNN

Proc. N. Engl. Zoöl. Club, Vol. 8, Feb. 27, 1923, p. 39.

Desmognathus monticola STEJNEGER and BARBour

Check List N. Amer. Amph. Rept., Ed. 1, 1917, p. 22. 
TYPE LOCALity: Taylor's Creek near Newport, Kentucky.

RANGE: Appalachian Mt. ranges from Pennsylvania southward to

Toccoa, Georgia and Newport, Kentucky.

Desmognathus quadra-maculatus (HoLBRook)

Salamandra quadra-maculata НоLвRоок

N. Amer. Herp., Ed. 1, Vol. 4, 1840, pl. 26 misprinted 27. (The text, p. 121, reads maculo-quadrata.)

Desmognathus quadrimaculata STEJNEGER

Proc. U. S. Nat. Mus., Vol. 26, Jan. 29, 1903, p. 557.

TyPe locality: Georgia and the Carolinas.

RANGE: Virginia to Georgia, in the mountains, 2000-6000 feet.

Leurognathus ( $G E N U S$ )

Moore, Proc. Ac. Nat. Sci. Phila., 1899, p. 316

TYPE: marmorata

Leurognathus marmorata Moore

Leurognathus marmorata MOoRE

Proc. Ac. Nat. Sci. Phila., 1899, p. 316.

TYPE LOCALity: South Slope Grandfather Mt., North Carolina, 3500 feet.

RANGE: Grandfather and Roan Mountains in North Carolina, and Lee Mountain, South Carolina.

\section{Leurognathus marmorata intermedia POPE}

Leurognathus marmorata intermedia Pope

Amer. Mus. Nov., No. 306, April 14, 1928, pp. 1-19.

TYPE LOCAlity: Davis Gap, Waynesville, North Carolina.

RANGE: Lee Mountain, North Carolina.

\section{MEANTES (SUBORDER)}

Linné, Syst. Nat., Ed. 12, Vol. 1, Pt. 2, 1766, sign. Rrrr 5, Addenda (not paged)

SIRENIDAE (FAMILY)

Siren ( $G$ E N $U S$ )

Linné, Syst. Nat., Ed. 12, Vol. 1, Pt. 2. 1766, sign. Rrrr 5, Addenda (not paged) TY PE: lacertina 
Siren intermedia LE Conte

Siren intermedia LE Conte

Journ. Ac. Nat. Sci. Phila., Vol. 5, Pt. 2, 1827, p. 322. ${ }^{1}$

Siren intermedia Noble and Marshall

Amer. Mus. Nov., No. 532, 1932, p. 1.

TYPE LOCALITY: "Southern States."

RANGE: Eastern Virginia to Florida, through the Gulf States west

to Texas.

Siren lacertina LINNÉ

MUD-EEL

Siren lacertina LinNÉ

Syst. Nat., Ed. 12, Vol. 1, Pt. 2, 1766, sign. Rrrr 5, Addenda (not paged).

Siren lacertina CopE

Bull. U. S. Nat. Mus., No. 34, 1889, p. 226, fig. 57.

TYPE LOCALITY: "Habitat in Carolinae paludosis."

RANGE: Eastern Virginia to Florida, through the Gulf States to

the west side of the Rio Grande; Mississippi Valley to Illinois

and Indiana; in scattered localities in Arkansas and Missouri.

\author{
Pseudobranchus ( $G E N U S$ ) \\ GraY, Ann. Philos., N. S., Vol. 10, 1825, p. 216 \\ TYPE: striatus
}

\title{
Pseudobranchus striatus (Le Conte)
}

Siren striata LE CONTE

Ann. Lyc. Nat. Hist. New York, Vol. 1, Pt. 1, 1824, p. 53, pl. 4.

Pseudobranchus striatus GRAY

Ann. Philos., N. S., Vol. 10, 1825, p. 216.

Pseudobranchus striatus COPE

Bull. U. S. Nat. Mus., No. 34, 1889, p. 230, fig. 58.

TYPE LOCALITY: Not given, but by inference it was one of the

Le Conte plantations in Floyd or Liberty County, Georgia.

RANGE: Swamps of southern South Carolina, lower Georgia and

Florida.

1 Not as cited by Noble, Amer. Mus. Nov., No. 532, 2 June 1932, p. 2. 


\section{SALIENTIA (ORDER)}

Laurenti, Syn. Rept., 1768, p. 24

\section{COSTATA (SUBORDER)}

Lataste, Act. Soc. Linn. Bordeaux, Vol. 33, 1879, p. 339

\section{ASCAPHIDAE (FAMILY)}

Ascaphus ( $G E N U S$ )

Stejneger, Proc. U. S. Nat. Mus., Vol. 21, June 20, 1899, p. 899 TYPE: truei

\section{Ascaphus truei Stejneger}

Ascaphus truei STEJNEGER

Proc. U. S. Nat. Mus., Vol. 21, June 20, 1899, p. 900, figs. 1-4, pl. 89. TYPE LOCALITY: Humptulips, Washington.

RANGE: Chehalis County and Pierce County, Washington, Mt.

Rainier in the Cascade Range, the Olympic Mountains, south to Humboldt County, California and east to Montana.

\section{LINGUA'TA (SUBORDER)}

Gravenhorst, Thierreich, 1845, p. 43

SCAPHIOPODIDAE (FAMILY)

\section{Scaphiopus ( $G E N U S$ )}

НоцвRоок, N. Amer. Herp., Ed. 1, Vol. 1, 1836, p. 85

TYPE: solitarius $=$ holbrookii

\section{Scaphiopus couchii BAIRD}

Scaphiopus couchii BAIRD

Proc. Ac. Nat. Sci. Phila., Vol. 7, 1854, p. 62.

Scaphiopus couchii COPE

Bull. U. S. Nat. Mus., No. 34, 1889, p. 301, fig. 75.

TYPE LOCALity: Rio Nasas, Coahuila, and Matamoros, Tamaulipas, Mexico.

RANGE: Texas to Arizona, northern states of Mexico, Lower

California. 


\section{Scaphiopus hammondii BAIRD}

Scaphiopus hammondii BAIRD

Rep. Pacif. R. R. Surv., Vol. 10, Williamson's Routc, Pt. 4, 1859, p. 12, pl. 28, fig. 2.

Spea hammondii COPE

Bull. U. S. Nat. Mus., No. 34, 1889, p. 303, fig. 77.

TyPE Locality: Fort Reading, California.

RANGE: Western and southwestern states from Montana south to

Texas and Mexico and westward to the Pacific coast states and northern Lower California.

\section{Scaphiopus holbrookii holbrookii (HaRLAN)}

SPADEFOOT

Rana holbrookii HARLAN

Med. Phys. Researches, 1835, p. 105.

Scaphiopus hurterii STRECKER

Stejneger and Barbour, Check List N. Amer. Amph. Rept., Ed. 2, 1923, p. 23.

Scaphiopus holbrookii CoPE

Bull. U. S. Nat. Mus., No. 34, 1889, p. 298.

TYPE LOCALITY: South Carolina.

RANGE: Eastern states, Massachusetts to Florida, west to Louisiana, Texas to Arkansas.

\section{Scaphiopus holbrookii albus (GARMAN)}

Scaphiopus albus Garman

Proc. Amer. Assoc. Adv. Sci., Buffalo, 1877, p. 194.

Scaphiopus holbrookii albus STEJNEger and BARBour

Check List N. Amer. Amph. Rept., Ed. 1, 1917, p. 26.

TYPE LOCALITY: Key West, Florida.

RANGE: Florida Keys and possibly the extreme southern part of

the peninsula of Florida.

\section{BUFONIDAE (FAMILY) \\ Bufo ( $G E N U S$ ) \\ LaUrenti, Syn. Rept., 1768, p. 25 \\ TYPE: vulgaris $=$ bufo}

\section{Bufo alvarius GirARD}

Bufo alvarius Glrard

U. S. Mex. Bound. Surv., Vol. 2, 1859, Rept., p. 26, pl. 41, figs. 1-6. Bufo alvarius Cope

Bull. U. S. Nat. Mus., No. 34, 1889, p. 265, fig. 62. 
TYPE LOCALITY: Valley of the Gila and Colorado Rivers.

RANGE: Southern Arizona and southern California.

Bufo americanus americanus HoLBrook

AMERICAN TOAD

Bufo americanus HolbrooK

N. Amer. Herp., Ed. 1, Vol. 1, 1836, p. 75, pl. 2.

Bufo lentiginosus americanus COPE

Bull. U. S. Nat. Mus., No. 34, 1889, p. 284, fig. 70.

TYPE LOCALITy: "From Maine through all the Atlantic States." RANGE: Eastern North America from Hudson Bay to Alberta and Labrador southward.

Bufo americanus copei Yarrow and Henshaw

Bufo americanus copei YarRow and HeNshaw

Rept. Rep. \& Batr. Cal. Ariz. Nev., 1878, p. 4.

Bufo americanus copei GAIGE

Copeia, 1932, No. 3, p. 134.

TYPE LOCALITy: James Bay, Ontario.

RANGE: Northern Ontario.

Bufo boreas boreas (BAIRD and GIRARD)

Bufo boreas BAIRD and Girard

Proc. Ac. Nat. Sci. Phila., Vol. 6, 1852, p. 174.

Bufo columbiensis COPE

Bull. U. S. Nat. Mus., No. 34, 1889, p. 267, fig. 63.

ty Pe Locality: Columbia River and Puget Sound.

RANGe: Colorado north and west to Puget Sound. Also British

Columbia and southwestern Alaska to Prince William Sound.

Bufo boreas halophilus (Baird and Girard)

Bufo halophila BAIRD and GIRARD

Proc. Ac. Nat. Sci. Phila., Vol. 6, 1853, p. 301.

Bufo boreas halophilus CAMP

Univ. California Publ. Zool., Vol. 17, No. 9, Feb. 3, 1917, p. 116.

Bufo columbiensis halophilus COPE

Bull. U. S. Nat. Mus., No. 34, 1889, p. 269, fig. 64.

TyPE LOCALITy: Benicia, California.

RANGE: Western Nevada, south central California, south to northern Lower California.

\section{Bufo californicus (CAMP)}

Bufo cognatus californicus CAMP

Univ. California Publ. Zool., Vol. 12, April 2, 1915, p. 331. 
Bufo californicus MYers

Proc. Biol. Soc. Wash., Vol. 43, Mch. 12, 1930, p. 73.

ty pe locality: Sta. Paula, Ventura Co., California.

RANGE: Extreme southern California and northern Lower California.

Bufo canorus CAMP

Bufo canorus CAMP

Univ. California Publ. Zool., Vol. 17, No. 6, Nov. 17, 1916, p. 59.

т Y PE Locality: Porcupine Flat, Yosemite National Park, California.

RANGE: Region about Porcupine Flat, Yosemite National Park,

California.

Bufo cognatus ( $\mathrm{S}_{\mathrm{AY}}$ )

Bufo cognatus $\mathrm{SAY}$

Long's Exp. Rocky Mts., Vol. 2, 1823, p. 190.

Bufo cognatus CoPE

Bull. U. S. Nat. Mus., No. 34, 1889, p. 275, fig. 67.

Ty PE Locality: Arkansas River, Prowers Co., Colorado.

RANGE: Arizona, Utah, and Colorado to Texas, Arkansas to Kansas, southern Wyoming, and North Dakota.

\section{Bufo compactilis WiEgManN}

Bufo compactilis WiegmaNN

Isis, 1833, p. 661.

Bufo compactilis CoPE

Bull. U. S. Nat. Mus., No. 34, 1889, p. 272, fig. 65.

TYPE LOCALITY: Mexico.

RANGE: Northern Mexico, Texas, New Mexico, and Arizona.

\section{Bufo debilis Girard}

Bufo debilis GIRARD

Proc. Ac. Nat. Sci. Plila., Vol. 7, 1854, p. 87.

Bufo debilis COPE

Bull. U. S. Nat. Mus., No. 34, 1889, p. 264, fig. 61.

TYPE LOCALITY: Lower part of the valley of the Rio Grande,

Texas, and in Tamaulipas, Mexico.

RANGE: Colorado south into the northern states of Mexico.

\section{Bufo fowleri HinckLeY}

FOWLER'S TOAD

Bufo fowleri HinckLex

Proc. Boston Soc. Nat. Hist., Vol. 21, 1882, p. 310. 
Bufo lentiginosus fowleri COPE

Bull. U. S. Nat. Mus., No. 34, 1889, p. 279, fig. 68.

TYPE LOCALITy: Milton, Massachusetts.

RANGE: New England and New York, southward to Georgia, west to Michigan and Missouri, but occurring also on the Atlantic and Gulf coastal plain to central Texas. (Not known in peninsular Florida.)

\section{Bufo hemiophrys CoPE}

Bufo hemiophrys Cope

Proc. Amer. Philos. Soc., Vol. 23, 1886, p. 515.

Bufo hemiophrys Cope

Bull. U. S. Nat. Mus., No. 34, 1889, p. 273, fig. 66 .

TYPE LOCality: Pembina and Turtle Mountains, North Dakota.

RANGE: Alberta, Manitoba, Northwest Territory, and North Dakota.

\section{Bufo marinus (LINNÉ)}

Rana marina LINNÉ

Syst. Nat., Ed. 10, 1758, Vol. 1, p. 211.

Bufo marinus SCHNEIDER

Hist. Amph., Pt. 1, 1799, p. 219.

Bufo marinus Boulenger

Cat. Batr. Sal. Brit. Mus., 1882, p. 315.

TyPe locality: America.

RANGE: Central and Tropical South America, entering the United

States about Brownsville, Texas.

\section{Bufo punctatus BAIRD and GIRARD}

Bufo punctatus BATrd and Girard

Proc. Ac. Nat. Sci. Phila., Vol. 6, 1852, p. 173.

Bufo punctatus Cope

Bull. U. S. Nat. Mus., No. 34, 1889, p. 262, fig. 60.

TyPe LOCAlity: Rio San Pedro, tributary of the Rio Grande del

Norte, Texas.

RANGE: Western Texas, Arizona, Utah, Colorado, southern California, and Lower California.

\section{Bufo quercicus Holbrook}

Bufo quercicus HoLBrook

N. Amer. Herp., Ed. 1, Vol. 4, 1840, p. 109, pl. 22.

Bufo quercicus Cope

Bull. U. S. Nat. Mus., No. 34, 1889, p. 291, fig. 72. 
Type locality: Charleston, South Carolina, and Smithville, North Carolina.

RANGE: North Carolina to Alabama and Florida, and west to Louisiana.

Bufo terrestris (Bonnaterre)

SOUTHERN TOAD

Rana terrestris BoNNATERRE

Tabl. Encycl. Méth., Erp., 1789, p. 8.

Bufo lentiginosus lentiginosus COPE

Bull. U. S. Nat. Mus., No. 34, 1889, p. 289, fig. 71.

TYPE LOCALitY: "La Caroline."

RANGE: The Carolinas to Florida, west to the Mississippi.

Bufo valliceps WiegmanN

Bufo valliceps WiegmanN

Isis, 1833, p. $65 \%$.

Bufo valliceps CopE

Bull. U. S. Nat. Mus., No. 34, 1889, p. 292, fig. 73.

TYPE LOCALITY: Mexico.

RANGe: Louisiana, New Mexico, Texas south to Costa Rica.

Bufo woodhousii GIRARD

Bufo woodhousii Girard

Proc. Ac. Nat. Sci. Phila., Vol. 7, 1854, p. 86.

Bufo lentiginosus woodhousei CopE

Bull. U. S. Nat. Mus., No. 34, 1889, p. 281, fig. 69.

TYPE LOCALITY: "New Mexico" = San Francisco Mountain,

Coconino County, Arizona.

RANGE: Texas to Kansas, Nebraska and Montana, west to Nevada, Arizona, and southeastern California.

\section{HYLIDAE (FAMILY)}

$$
\text { Acris ( } G E N U S \text { ) }
$$

Duméril and Bibron, Erp. Gén., Vol. 8, 1841, p. 506 TYPE: gryllus

Acris gryllus (Le ConTe)

CRICKET-FROG

Rana gryllus LE CoNTE

Ann. Lyc. Nat. Hist., New York, Vol. 1, Pt. 2, 1825, p. 282. Acris gryllus Duméril and BibroN

Erp. Gén., Vol. 8, 1841, p. $50 \%$. 
Acris gryllus COPE

Bull. U. S. Nat. Mus., No. 34, 1889, p. 324, fig. 82.

TYPE LOCALITY: Not given.

RANGE: Florida to New York in the east; northward through the central valley from Louisiana and Texas to the Canadian Northwest Territories.

\section{Pseudacris ( $G E N U S$ )}

Fitzinger, Syst. Rept., 1843, p. 31

TYPE: nigrita

Pseudacris brachyphona (CoPE)

Chorophilus feriarum brachyphonus CoPE

Bull. U. S. Nat. Mus., 1889, No. 34, p. 341.

Pseudacris brachyphona W WLKER

Ohio Journ. Sci., Vol. 32, No. 4, 1932, p. 379.

Type locality: "West Pennsylvania, near the Kiskiminitas River."

RANGE: Eastern Ohio, West Virginia, western Pennsylvania and western Maryland.

\section{Pseudacris nigrita nigrita (LE CONTE)}

Rana nigrita Le Conte

Ann. Lyc. Nat. Hist., New York, Vol. 1, Pt. 2, 1825, p. 282.

Pseudacris nigrita GüNTHER

Cat. Batr. Sal. Brit. Mus. 1858, p. 97.

Chorophilus nigritus Cope

Bull. U. S. Nat. Mus., No. 34, 1889, p. 337, fig. 85.

TYPE LOCALITY: Not given.

RANGE: South Carolina, south through Florida, west to Missisippi.

Pseudacris nigrita clarkii (BAIRD)

Helocaetes clarkii BAIRD

Proc. Ac. Nat. Hist., Phila., Vol. 7, 1854, p. 60.

TYPE LOCALITY: Galveston and Indianola, Texas:

RANGE: Same.

Pseudacris nigrita feriarum (BAIRD)

Helocaetes feriarum BATRD

Proc. Ac. Nat. Sci. Phila., Vol. 7, 1854, p. 60.

Pseudacris feriarum CoPE

Proc. Ac. Nat. Sci. Phila., 1862, p. 157. 
Chorophilus feriarum CoPE

Bull. U. S. Nat. Mus., No. 34, 1889, p. 339, fig. 86 .

ty pe locality: Carlisle, Pennsylvania.

RANGE: Eastern United States, west to Illinois.

Pseudacris nigrita occidentalis (BAIRD and GIRARD)

Litoria occidentalis BAIRD and GIRARD

Proc. Ac. Nat. Sci. Phil., Vol. 6, 1853, p. 301.

Pseudacris occidentalis Stejneger and Barbour

Check List N. Amer. Amph. Rept., Ed. 1, 1917, p. 30.

Chorophilus occidentalis CopE

Bull. U. S. Nat. Mass., No. 34, 1889, p. 335.

TYPE LOCALITY: The type was incorrectly stated to have come

from San Francisco.

Range: Arkansas and Texas.

Pseudacris nigrita septentrionalis (Boulenger)

Chorophilus septentrionalis Boulenger

Cat. Batr. Sal. Brit. Mus., 1882, p. 335, pl. 23, fig. 1.

Pseudacris septentrionalis STEJNEGER and BARBour

Check List N. Amer. Amph. Rept., Ed. 1, 1917, p. 31.

TYPE LOCALITY: Great Bear Lake, Canada.

RANGE: Canadian northwestern swamp areas.

Pseudacris nigrita triseriata (WIED)

Hyla triseriata WIED

Reise Nord-Amer., Vol. 1, Pt. 4, 1838, p. 249.

Pseudacris triseriata Stejneger and Barbour.

Check List N. Amer. Amph. Rept., Ed. 1, 1917, p. 31.

Chorophilus triseriatus CoPE

Bull. U. S. Nat. Mus., No. 34, 1889, p. 342, fig. 87.

ty PE Locality: Mt. Vernon, Ohio River, Indiana.

RANGE: Allegheny Mts. to New Mexico, Arizona, and Idaho.

Pseudacris ocularis (HoLBRooK)

Hylodes ocularis HoLBrooK

N. Amer. Herp., Ed. 1, Vol. 3, 1838, p. 79, pl. 14.

Pseudacris ocularis STEJNEGER and BARBOUR

Check List N. Amer. Amph. Rept., Ed. 1, 1917, p. 31.

Chorophilus ocularis COPE

Bull. U. S. Nat. Mus., No. 34, 1889, p. 348.

TYPE LOCALITY: South Carolina and Georgia.

RANGE: Southeastern United States. 


\section{Pseudacris ornata (НоLвRоoк)}

Rana ornata HoLbrook

N. Amer. Herp., Ed. 1, Vol. 1, 1836, p. 97, pl. 16.

Pseudacris ornata STEJNEgER and BARBOUR

Check List N. Amer. Amph. Rept., Ed. 1, 1917, pl. 31.

Chorophilus ornatus CoPE

Bull. U. S. Nat. Mus., No. 34, 1889, p. 333.

TyPE Locality: Between the Cooper and Ashley Rivers, four miles from Charleston, South Carolina.

RANGE: North Carolina to Florida.

\section{Hyla ( $G E N U S$ )}

LAURENTI, Syn. Rept., 1768, p. 32 TYPE: viridis

Hyla andersonii BAIRD

ANDERSON TREE-FROG

Hyla andersonii BAIRD

Proc. Ac. Nat. Sci. Phila., Vol. 7, 1854, p. 60.

Hyla andersonii COPE

Bull. U. S. Nat. Mus., No. 34, 1889, p. 365, fig. 91, pl. 83, fig. 1.

tyPe locality: Anderson, South Carolina.

RANGE: White cedar swamps in southern New Jersey, eastern

Pennsylvania, and South Carolina.

\section{Hyla arenicolor Cope}

Hyla arenicolor COPE

Journ. Ac. Nat. Sci. Phila., Ser. 2, Vol. 6, July, 1866, p. 84.

Hyla arenicolor COPE

Bull. U. S. Nat. Mus., No. 34, 1889, p. 369, fig. 93.

TYPE LOCALITY: Northern Sonora, Mexico.

RANGE: Most of the southwestern United States from Utah to northern Lower California, east to Texas, and in Mexico south to Guadalajara and Toluca.

\section{Hyla avivoca Viosca}

Hyla avivoca Viosca

Proc. Biol. Soc. Washington, Vol. 41, June 29, 1931, p. 89.

TYPE LOCALITy: Mandeville, Louisiana.

RANGE: Eastern Louisiana north to Tennessee and southern Illinois; east to Florida. 
Hyla baudinii baudinii (Duméril and Bibron)

Iyla baudinii Duméril and Bibron

Erp. Gén., Vol. 8, 1841, p. 564.

Smilisca baudinii COPE

Bull. U. S. Nat. MLus., No. 34, 1889, p. 379.

TYPE LOCALITY: Mexico.

RANGE: Central America north to southern Texas.

Hyla cinerea cinerea (SchNeIDER)

Calamita cinereus SchNeIDER

Hist. Amph., Vol. 1, 1799, p. 174.

IIyla cinerea GARMAN

Bull. Ill. State Lab. Nat. Hist., Vol. 3, 1892, p. 349.

Iyla carolinensis Cope

Bull. U. S. Nat. Mus., No. 34, 1889, p. 366, fig. 92.

TYPE LOCALITY: "Inhabits Carolina."

RANGE: Virginia to Florida, west to Texas and northward up the

Mississippi Valley to southern Illinois.

\section{Hyla cinerea evittata (Miller)}

Hyla evittata MILLER

Proc. Biol. Soc. Washington, Vol. 13, 1899, p. 75.

Hyla cinerea evittata DunN

Copeia, No. 53, 1918, p. 21.

TyPe LOCALity: Four Mile Run, Virginia (near the City of

Washington).

RANGe: Reed beds and lily pools along the Potomac and York

Rivers, Virginia.

Hyla crucifer WIED

SPRING-PEEPER

Hyla crucifer WIED

Reise Nord-Amer., Vol. 1, Pt. 5, 1838, p. 275.

Hyla pickeringii COPE

Bull. U. S. Nat. Mus., No. 3t, 1889, p. 352, fig. 88.

TYPE LOCALITY: Cantonment Leavenworth, "Kansas."

RANGE: New Brunswick to Manitoba, south to Georgia, Louisiana, Arkansas, and Kansas.

\section{Hyla eximia BAIRD}

Hyla eximia BAIRD

Proc. Ac. Nat. Sci. Phila., Vol. 7, 1854, p. 61.

Hyla eximia Cope

Bull. U. S. Nat. Mus., No. 34, 1889, p. 361. 
TYPE LOCALity: City of Mexico.

RANGE: Mexico, Texas, New Mexico, and Arizona.

\section{Hyla femoralis LATREILLE}

Hyla femoralis LATREILLE

Hist. Nat. Rept., Vol. 2, 1802, p. 181.

Hyla femoralis COPE

Bull. U. S. Nat. Mus., No. 34, 1889, p. 371, fig. 94.

TYPE LOCAlity: Carolina.

RANGE: Carolinas to Florida and west to Texas.

\section{Hyla gracilipes CoPE}

IIyla gracilipes COPE

Proc. Ac. Nat. Sci. Phila., Vol. 17, 1865, p. 194.

Hyla gracilipes KeLLoG

Bull. U. S. Nat. Mus., No. 160, 1932, p. 168.

ty le locality: Mirador, Vera Cruz, Mexico.

RANGE: Southern Arizona, and New Mexico southward to Vera

Cruz and the Valley of Mexico.

\section{Hyla gratiosa Le Conte}

\section{Hyla gratiosa Le Conte}

Proc. Ac. Nat. Sci. Phila., 1856, p. 146, pl. 4.

Hyla gratiosa CoPE

Bull. U. S. Nat. Mus., No. 34, 1889, p. 377, fig. 96, pl. 83, fig. 5 .

TYPE LOCALITY: "Lower country of Georgia," evidently one of

the Le Conte plantations in Floyd or Liberty County.

range: South Carolina to Florida, Mississippi, and Louisiana.

\section{Hyla regilla BaIrd and GIRARD}

PACIFIC TREE-TOAD

Hyla regilla BAIRD and GIRARD

Proc. Ac. Nat. Sci. Phila., Vol. 6, 1852, p. 174.

Hyla regilla CoPe

Bull. U. S. Nat. Mus., No. 34, 1889, p. 355, fig. 89.

TyPE LOCAlity: "Sacramento River, in Oregon and Puget Sound."

RANGE: Vancouver Island and British Columbia to Lower California; Idaho, Nevada and Arizona.

\section{Hyla septentrionalis BOULENGER}

Hyla septentrionalis BoULENGER

Cat. Batr. Sal. Brit. Mus., 1882, p. 368. 
TYPE LOCAlity: Cuba and San Domingo. [Restricted to Cuba.] range: Cuba, Bahama Islands and Key West (recently introduced).

Hyla squirella LATreiLle

Hyla squirella LATREILLE

Hist. Nat. Rept., Vol. 2, 1802, p. 181.

IIyla squirella Cope

Bull. U. S. Nat. Mus., No. 34, 1889, p. 363, fig. 90.

TyPE LOCALITy: Carolina.

RANGE: Virginia to Florida, west to Texas northward up the

Mississippi basin to Indiana.

\section{Hyla versicolor versicolor (LE CONTE)}

TREE-TOAD, RAIN-TOAD

Hyla versicolor Le Conte

Ann. Lyc. Nat. Hist., New York, Vol. 1, Pt. 2, 1825, p. 281.

Hyla versicolor COPE

Bull. U. S. Nat. Mus., No. 34, 1889, p. 373, fig. 95.

TYPE LOCALITY: “Northern States."

RANGE: Maine, southern Canada, west to Minnesota, south to the Gulf States (Texas and Arkansas in part only).

\section{Hyla versicolor chrysoscelis (COPE)}

Hyla femoralis chrysoscelis Cope

Bull. U. S. Nat. Mus., No. 17, 1880, p. 29.

IIyla versicolor chrysoscelis STRECKER

Proe. Biol. Soe. Washington, Vol. 33, 1910, p. 117.

TyPe LOCAlity: Dallas, Texas.

RANGE: Southern Arkansas to east-central Texas.

\section{LEPTODACTYLIDAE (FAMILY)}

Leptodactylus ( $G E N U S$ )

Fitzinger, Neue Classif. der Rept., 1826, p. 38 TYPE: typhonia

\section{Leptodactylus albilabris (GüNTHER)}

Cystignathus albilabris GÜNTHER

Ann. Mag. Nat. Hist., Ser. 3, Vol. 4, 1859, p. 217. 
Leptodactylus albilabris Boulenger

Bull. Soc. Zool. France, 1881, p. 33.

Leptodactylus albilabris TAYLOR

Univ. Kansas Sci. Bull., Vol. 20, No. 11, 1932, p. 243.

TYPE LOCALITY: St. Thomas.

RANGE: West Indies, Mexico and Los Olmos bridge near Rio

Grande City, Texas.

\section{Eleutherodactylus ( $G E N U S$ )}

Duméril and Bibron, Erp. Gén., Vol. 8, 1841, p. 620

TYPE: martinicensis

\section{Eleutherodactylus augusti (DUG Ès)}

Hylodes augusti Dugès

In Brocchi, Bull. Soc. Philom. Paris, Ser. 7, Vol, 3, 1879, No. 1, p. 21.

Eleutherodactylus augusti SLEVIN

Copeia, 1931, No. 3, p. 140.

TyPe locality: Guanajuato, Mexico.

RANGE: Central Mexico north to southern Arizona in the highlands.

\section{Eleutherodactylus latrans (COPE)}

Lithodytes latrans CoPE

Bull. U. S. Nat. Mus., No. 17, 1880, p. 25.

Eleutherodactylus latrans STEJNEGER and BARBour

Check List N. Amer. Amph. Rept., Ed. 1, 1917, p. 34.

Lithodytes latrans Cope

Bull. U. S. Nat. Mus., No. 34, 1889, p. 316, fig. 80.

TyPE LOCAlity: Helotes, Bexar Co., Texas.

RANGE: Texas.

Eleutherodactylus ricordii (DUMÉRIL and BIBRON)

IIylodes ricordii Duméril and Bibron

Erp. Gén., Vol. 8, 1841, p. 623.

Eleutherodactylus ricordii STEJNEGER

Shattuck's Bahama Islands, 1905, p. 331.

Lithodytes ricordii CopE

Bull. U. S. Nat. Mus., No. 34, 1889, p. 318.

TYPE LOCALITY: Cuba.

RANGE: Bahama Islands, Cuba and Florida, north to Alachua

County (probably accidentally introduced). 


\section{Syrrhophus ( $G E N U S$ ) \\ Cope, Amer. Nat., Vol. 12, 1878, p. 253 \\ TYPE: marnockii}

\section{Syrrhophus campi STEJNEGER}

Syrrhophus campi STEJNEgER

Proc. Biol. Soc. Washington, Vol. 28, June 29, 1915, p. 131.

TYPE LOCALITY: Brownsville, Texas.

RANGE: At present known from the type locality only.

\section{Syrrhophus marnockii COPE}

Syrrhophus marnockii COPE

Amer. Nat., Vol. 12, 1878, p. 253.

Syrrhophus marnockii COPE

Bull. U. S. Nat. Mus., No. 34, 1889, p. 318, fig. 81.

Ty PE Locality: Near San Antonio, Texas.

RANGE: Bexar, Hays, Travis, and Brewster Counties, Texas.

\section{RANIDAE (FAMILY) \\ Rana ( $G E N U S)$}

LinNé, Syst. Nat., Ed. 10, Vol. 1, 1758, p. 210

TYPE: temporaria

Rana aesopus (CoPE)

GOPHER-FROG

Rana areolata aesopus COPE

Proc. Amer. Philos. Soc., Vol. 23, Dec. 1886, p. 517.

Rana aesopus Stejneger and BARBoUr

Check List N. Amer. Amph. Rept., Ed. 1, 1917, p. 36.

Rana areolata aesopus Cope

Bull. U. S. Nat. Mus., No. 34, 1889, p. 412.

TYPE LOCAlity: Micanopy, Florida.

RANGE: Florida northward into South Carolina.

Rana areolata BAIRD and GIRARD

Rana areolata BAIRD and GIRARD

Proc. Ac. Nat. Sci. Phila., Vol. 6, 1852, p. 173.

Rana areolata Cope

Bull. U. S. Nat. Mus., No. 34, 1889, p. 409, fig. 103.

TYPE LOCALITY: Indianola, Texas.

RANGE: Georgia, Texas, Missouri, Oklahoma, Indiana, and Illinois. 
Rana aurora aurora (BAIRD and GIRARD)

Rana aurora BAIRD amd GIRARD

Proc. Ac. Nat. Sci. Phila., Vol. 6, 1852, p. 174.

Rana aurora aurora CAMP

Univ. California Publ. Zool., Vol. 17, 1917, p. 123.

Rana agilis aurora COPE

Bull. U. S. Nat. Mus., No. 34, 1889, p. 439, fig. 113.

TyPe Locality: Puget Sound.

RANGE: California to Washington, Vancouver Island.

Rana aurora draytonii (BAIRD and GIRARD)

Rana draytonii BAIRD and GIRARD

Proc. Ac. Nat. Sci. Phila., Vol. 6, 1852, p. 174.

Rana aurora draytonii CAMP

Univ. California Publ. Zool., Vol. 17, No. 9, Feb. 3, 1917, p. 115.

Rana draytoni draytoni COPE

Bull. U. S. Nat. Mus., No. 34, 1889, p. 441, fig. 114.

ty PE locality: San Francisco, and the Columbia River.

RANGE: Oregon, California, and Lower California.

Rana boylii boylii (BAIRD)

Rana boylii BAIRD

Proc. Ac. Nat. Sci. Phila., Vol. 7, 1854, p. 62.

Rana boylii boylii CAMP

Univ. California Publ. Zool., Vol. 17, No. 9, Feb. 3, 1917, p. 117.

Rana boylii COPE

Bull. U. S. Nat. Mus., No. 34, 1889, p. 444, fig. 115.

ty ye locality: El Dorado County, California.

RANGE: California and southwestern Oregon.

Rana boylii muscosa CAMP

Rana boylii muscosa CAMP

Univ. California Publ. Zool., Vol. 17, No. 9, Feb. 3, 1917, p. 118.

TyPe LOCALity: Arroyo Seco Cañon, at about 1300 feet altitude, near Pasadena, California.

RANGE: California: San Gabriel, San Bernardino, and San Jacinto Mountains, south to San Diego County.

Rana boylii sierrae CAMP

Rana boylii sierrae CAMP

Univ. California Publ. Zool., Vol. 17, No. 9, Feb. 3, 1917, p. 120.

TYPE Locality: Matlack Lake, Sierra Nevada, Inyo County,

California, 10,500 feet altitude.

RANGE: Southern half of Sierra Nevada, above $\mathbf{7 0 0 0}$ feet altitude,

California. 


\section{Rana cantabrigensis BAIRD}

Rana cantabrigensis $\mathrm{B}_{\mathrm{AIRD}}$

Proc. Ac. Nat. Sci. Phila., Vol. 7, 1854, p. 62.

Rana cantabrigensis latiremis STEJNEGER and BARBour

Check List N. Aner. Amph. Rept., Ed. 1, 1917, p. 37.

Rana cantabrigensis cantabrigensis $\mathrm{COPE}$

Bull. U. S. Nat. Mus., No. 34, 1889, p. 436, fig. 110.

TyPe locality: Cambridge, Mass. (in errore).

RANGE: Alaska, through the lowlands eastward to Michigan.

\section{Rana catesbeiana SHAw}

\section{BULLFROG}

Rana catesbeiana Shaw

Gen. Zool., Vol. 3, Pt. 1, 1802, p. 106, pl. 33.

Rana catesbiana CoPE

Bull. U. S. Nat. Mus., No. 34, 1889, p. 424, fig. 108.

TYPE LOCALITY: South Carolina.

RANGE: North America east of Rocky Mts. except extreme south-

east and Gulf states coastal plain. Introduced into California.

Rana clamitans LATrEILLE

GREEN FROG, SPRING-FROG

Rana clamitans LATREILLE

Hist. Nat. Rept., Vol. 2, 1802, p. 157.

Rana clamata Cope

Bull. U. S. Nat. Mus., No. 34, 1889, p. 419, fig. 107.

TYPE LOCALITY: Charleston, South Carolina.

RANGE: Eastern North America, Canada to Florida and Louisiana, west to Michigan, Wisconsin, Minnesota, Illinois, Arkansas and Texas.

\section{Rana grylio SteJNEGER}

Rana grylio STEJNEGER

Proc. U. S. Nat. Mus., Vol. 24, Oct. 4, 1901, p. 212.

ty PE LOCAlity: Bay St. Louis, Mississippi.

RANGE: Southern Louisiana to peninsular Florida, north to southeastern Georgia.

\section{Rana heckscheri WrIGHT}

Rana heckscheri WRIGHT

Proc. Biol. Soc. Wash., Vol. 37, Dec. 29, 1924, p. 143, pl. 11, 12, fig. 2. TYPE LOCALITY: Alligator Swamp, Callahan, Florida.

RANGE: Coastal Georgia, northern Florida and southern Mississippi. 


\section{Rana onca Cope}

Rana onca Cope

in Wheeler's Rep. Expl. W. 100th Mer., Vol. 5, Zoöl., 1875, p. 528, pl. 25, figs. 1-3.

Rana draytoni onca COPE

Bull. U. S. Nat. Mus., No. 34, 1889, p. 443.

TYPE LOCALITY: Utah.

RANGE: Utah and Nevada.

Rana palustris Le ConTe

PICKEREL-FROG

Rana palustris LE CONTE

Ann. Lyc. Nat. Hist., New York, Vol. 1, Pt. 2, 1825, p. 282.

Rana palustris Cope

Bull. U. S. Nat. Mus., No. 34, 1889, p. 406, fig. 102.

TYPE LOCALITY: Not given.

RANGE: Hudson Bay south to Arkansas and Louisiana and all of the Eastern States; west to Wisconsin.

\section{Rana pipiens ScHREBer}

\section{LEOPARD-FROG}

Rana pipiens SCHREBer

Naturforscher, Vol. 18, 1782, p. 185, pl. 4.

Rana virescens CoPE

Bull. U. S. Nat. Mus., No. 34, 1889, p. 397.

TYPE LOCALIty: Raccoon, Gloucester County, New Jersey.

RANGE: North America, east of the Sierra Nevada southward into

Mexico.

\section{Rana pretiosa pretiosa (BAIRD and GIRARD)}

Rana pretiosa BAIRD and GrRARD

Proc. Ac. Nat. Sci. Phila., Vol. 6, 1853, p. 378.

Rana temporaria pretiosa CopE

Bull. U. S. Nat. Mus., No. 34, 1889, p. 432, fig. 110.

ty yocality: Puget Sound, Washington.

RANGE: Utah and Montana west to the Pacific coast, California to British Columbia.

\section{Rana pretiosa luteiventris Thомpson}

Rana pretiosa luteiventris Thompson

Proc. Biol. Soc. Washington, Vol. 26, March 22, 1913, p. 53.

TyPe Locality: Anne Creek, Elko County, Nevada.

RANGE: Eureka and Elko Counties, Nevada. 


\section{Rana septentrionalis BAIRD \\ MINK-FROG \\ Rana septentrionalis $\mathrm{B}_{\mathrm{AIRD}}$}

Proc. Ac. Nat. Sci. Phila., Vol. 7, 1854, p. 61.

Rana septentrionalis CoPE

Bull. U. S. Nat. Mus., No. 34, 1889, p. 416, fig. 106, pl. 86.

TYPE LOCALITY: Northern Minnesota.

RANGE: Northern New England and northern New York, west

through Michigan to Minnesota, Canada to Hudson Bay.

Rana sphenocephala (COPE)

SOUTHERN LEOPARD-FROG

Rana halecina sphenocephala CopE

Proc. Amer. Philos. Soc., Vol. 23, Dec. 1886, p. 517.

Rana sphenocephala Stejneger and Barbour

Check List N. Amer. Amph. Rept., Ed. 1, 1917, p. 39.

Rana virescens sphenocephala COPE

Bull. U. S. Nat. Mus., No. 34, 1889, p. 399, fig. 99.

TyPE LOCALity: Near St. John's River, Florida.

RANGE: Southeastern States.

Rana sylvatica Le Conte

WOOD-FROG

Rana sylvatica LE CONTE

Ann. Lyc. Nat. Hist. New York, Vol. 1, Pt. 2, 1825, p. 282.

Rana silvatica CoPE

Bull. U. S. Nat. Mus., No. 34, 1889, p. 447, fig. 115 .

TYPE LOCALITY: Not stated.

RANGE: Quebec and Nova Scotia to South Carolina, westward to southern Ontario and Ohio.

\section{Rana tarahumarae BouLENGER}

Rana tarahumarae BOULENGER

Ann. Mag. Nat. Hist., Ser. 8, Vol. 20, 1917, p. 416.

TYPE LOCALITY: Sierra Tarahumare, northwestern Mexico.

RANGE: Chihuahua and southern Arizona in mountains, 3000 4000 feet.

Rana virgatipes Cope

SPIIAGNUM-FROG

Rana virgatipes CoPE

Amer. Nat., Vol. 25, 1891, p. 1017.

TYPE LOCAlity: Mare Run, near Great Egg Harbor, Atlantic

Co., New Jersey.

RANGE: New Jersey to the Okefinokee Swamp, Georgia. 


\section{BREVICIPITIDAE (FAMILY)}

Gastrophryne ( $G E N U S$ )

Fitzinger, Syst. Rept., 1843, p. 33

TYPE: rugosa $=$ carolinensis

Gastrophryne areolata (STRECKER)

Engystoma areolata (STRECKER)

Proc. Biol. Soc. Washington, Vol. 22, June 25, 1909, p. 118.

Gastrophryne areolata STEJNEGER and BARBOUR

Check List N. Amer. Ampl. Rept., Ed. 1, 1917, p. 40.

TYPE LOCALITY: Guadalupe River bottom, Victoria, Victoria Co., Texas.

RANGE: Southeastern Texas.

\section{Gastrophryne carolinensis (HoLBRook)}

Engystoma carolinense НоцвRоок

N. Amer. Herp., Ed. 1, Vol. 1, 1836, p. 83, pl. 11.

Gastrophryne carolinensis STEJNEGER and BARBOUR

Check List N. Amer. Amph. Rept., Ed. 1, 1917, p. 40.

Engystoma carolinense (part) CoPE

Bull. U. S. Nat. Mus., No. 34, 1889, p. 385, fig. 97.

Ty PE LOCALITY: Charleston, South Carolina.

RANGE: Virginia to Florida, Gulf States to Texas, northward

through central valley to southern Indiana.

Gastrophryne texensis (GIRARD)

Engystoma texense GIRARD

Proc. Ac. Nat. Sci. Phila., 1859, p. 169.

Gastrophryne texensis SteJNEGER and Barbour

Check List N. Amer. Amph. Rept., Ed. 1, 1917, p. 40.

Engystoma carolinense (part) COPE

Bull. U. S. Nat. Mus., No. 34, 1889, p. 385.

ty Pe locality: Rio Seco, near D’Hanis, Medina Co., Texas. RANGE: 'Texas north to Kansas.

\section{Hypopachus ( $G E N U S$ )}

Keferstein, Nachr. Ges. Wiss. Göttingen, July 24, 1867, p. 351 TYPE: seebachii $=$ variolosus

\section{Hypopachus cuneus COPE}

Hypopachus cuneus COPE

Proc. U. S. Nat. Mus., Vol. 11, July 5, 1889, p. 395.

Hypopachus cuneus COPE

Bull. U. S. Nat. Mus., No. 34, 1889, p. 388, fig. 98.

TyPE Locality: San Diego, Duval Co, Texas.

RANGE: Southern Texas. 


\title{
REPTILIA (CLASS)
}

\author{
Laurenti, Syn. Rept., 1768, p. 19
}

\section{DIAPSIDA (SUBCLASS)}

OsBorn, Science, N. S., Vol. 17, Feb. 13, 1903, p. 276

\author{
LORICATA (ORDER) \\ Merrem, Syst. Amph., 1820, p. 34 \\ CROCODYLIDAE (FAMILY) \\ Crocodylus ( $G E N U S$ ) \\ LaURenti, Syn. Rept., 1768, p. 53 \\ TYPE: niloticus = Lacerta crocodilus LINNÉ in part
}

\section{Crocodylus acutus Cuvier}

CROCODILE

Crocodilus acutus Cuvier

Ann. Mus. Hist. Nat. Paris, Vol. 10, 1807, p. 55, pl. 1, fig. 3, pl. 2, fig. 5.

Crocodilus americanus Cope

Ann. Rep. U. S. Nat. Mus., 1898 (1900), p. 173, fig. 2.

TyPE LOCAlity: San Domingo.

RANGE: Southern Florida, the Greater Antilles (except Porto

Rico), both coasts of Central America from Mexico to Ecuador and Colombia.

$$
\text { Alligator ( } G E N U S)
$$

Cuvier, Ann. Mus. Hist. Nat. Paris, Vol. 10, 1807, p. 25

TYPE: lucius = Lacerta alligator BLUMENBACH, 1788, in part = mississipiensis

Alligator mississipiensis (DAUDIN)

ALLIGATOR

Crocodilus mississipiensis DAUDIN

Hist. Nat. Rept., Vol. 2, 1803, p. 412. 
Alligator missisipensis [sic] GraY

Syn. Rept., 1831, p. 62.

Alligator mississippiensis CoPE

Ann. Rep. U. S. Nat. Mus., 1898 (1900), p. 168, fig. 1.

TYPE LOCALITy: "Les bords du Mississippi."

RANGE: Rivers and swamps of the lowlands of the Carolinas,

Georgia and Florida, west to Louisiana, Mississippi and westward to the Rio Grande in Texas.

\section{SQUA MATA (ORDER)}

Oppel, Ordn. Rept., 1811, p. 14

SAURIA (SUBORDER)

Macartney, in Ross's Transl. Cuvier's Lect. Comp. Anat., 1802, Vol. 1, table 3

GEKKONIDAE (FAMILY)

Hemidactylus ( $G E N U S$ )

Oken, Isis, 1817, p. 1183

TYPE: "Gecko tuberculeaux Daudin" = mabouia

Hemidactylus turcicus (LINNÉ)

Lacerta turcica LiNNÉ

Syst. Nat., Ed. 10, Vol. 1, 1758, p. 202.

Hemidactylus turcicus BoEtTGer

Ber. Offenbach. Ver. Naturk., 1876, p. 57.

TYPE LOCALITY: In Oriente.

RANGE: Borders of Red and Mediterranean Seas, northwestern

India; Key West. ${ }^{1}$

$$
\begin{aligned}
& \text { Phyllodactylus ( } G E N U S \text { ) } \\
& \text { GraY, Spicileg. Zool., 1830, p. } 3 \\
& \text { TYPE: pulcher }
\end{aligned}
$$

\section{Phyllodactylus tuberculosus WiegmanN}

Phyllodactylus tuberculosus WIEGManN

Nova Acta Ac. Leopold.-Carol., Vol. 17, 1835, p. 241, pl. 18, figs. 2-2a.

1 Accidentally introduced and possibly not established. 
Phyllodactylus tuberculosus CoPE

Ann. Rep. U. S. Nat. Mus., 1898 (1900), p. 458, fig. 83.

TYPE LOCALITY: "Californien."

RANGE: Ecuador to Lower California and extreme southern California.

\section{Phyllodactylus unctus CoPE}

Diplodactylus unctus COPE

Proc. Ac. Nat. Sci. Phila., 1863, p. 102.

Phyllodactylus (Diplodactylus) unctus Bocourt

Miss. Sci. Mex., Rept., Pt. 2, 1873, p. 43.

Phyllodactylus unctus CoPE

Ann. Rep. U. S. Nat. Mus., 1898 (1900), p. 460, fig. 84.

Ty PE LOCALity: Cape St. Lucas, Lower California.

RANGE: Cape region of Lower California and Islands in the Gulf off the southern portion of the Peninsula.

\section{Sphaerodactylus ( $G E N U S$ )}

WAgLer, Syst. Amph., 1830, p. 143

TYPE: sputator

Sphaerodactylus cinereus WAGLER

Sphaerodactylus cinereus WAGLER

Syst. Amph., 1830, p. 143.

TYPE LOCALITY: Haiti.

RANGe: Haiti, Cuba, Isla de Pinos, Key West. $^{1}$

Sphaerodactylus notatus BAIRD

Sphaerodactylus notatus BAIRD

Proc. Ac. Nat. Sci. Phila., 1858, p. 254.

Sphaerodactylus notatus COPE

Ann. Rep. U. S. Nat. Mus., 1898 (1900), p. 462, fig. 85.

TyPE LOCALity: Key West, Florida.

RANGE: Bahama Islands, Cuba, also southern Florida and the Keys (perhaps introduced by commerce).

1 Recently introduced accidentally. 


\title{
Coleonyx ( $G E N U S$ )
}

Gray, Ann. Mag. Nat. Hist., Vol. 16, 1845, p. 162

TYPE: elegans

Coleonyx brevis STEJNEGer

Coleonyx brevis STEJNeger

N. Amer. Fauna, No. 7, 1893, p. 163.

Eublepharis variegatus (part) CoPE

Ann. Rep. U. S. Nat. Mus., 1898 (1900), fig. 86.

TYPE LOCALity: Helotes, Bexar County, Texas.

RANGE: Texas.

\section{Coleonyx variegatus (BAIRD)}

Stenodactylus variegatus BAIRD

Proc. Ac. Nat. Sci. Phila., 1858, p. 254.

Coleonyx variegatus Cope

Proc. Ac. Nat. Sci. Phila., 1866, p. 125.

Eublepharis variegatus (part) CoPE

Ann. Rep. U. S. Nat. Mus., 1898 (1900), p. 466.

ty ye LOCALity: Rio Grande and Gila Valleys.

RANGE: Southwestern Utah, Texas to California, northern Lower

California, Cerros Island, Santa Ynez Island, and San Marcos

Island.

\author{
IGUANIDAE (FAMILY) \\ Anolis ( $G E N U S$ ) \\ Daudin, Hist. Nat. Rept., Vol. 4, 1803, p. 50 \\ TYPE: bullaris $=$ carolinensis
}

Anolis carolinensis VoigT

"CHAMELEON," ANOLIS

Anolius carolinensis Vorg't

Cuvier's Thierreich, Vol. 2, 1832, p. 71.

Anolis carolinensis COPE

Ann. Rep. U. S. Nat. Mus., 1898 (1900), p. 233, fig. 16.

TYPE LOCALITY: Carolina.

RANGE: North Carolina to Florida, westward through the Gulf region to the Rio Grande.

Anolis stejnegeri $\mathrm{B}_{\mathrm{ARBOUR}}$

Anolis stejnegeri $\mathrm{B}$ ARBoUR

Copeia, Oct. 30, 1931, 3, p. 88.

TyPE LOCALity: Key West, Florida.

RANGE: The Island of Key West. 


\section{Ctenosaura ( $G E N U S$ ) \\ WiegmanN, Isis, 1828, p. 371 \\ TYPE: cycluroides}

Ctenosaura hemilopha (COPE)

Cyclura (Ctenosaura) hemilopha CoPE

Proc. Ac. Nat. Sci. Phila., 1863, p. 105.

Ctenosaura hemilopha CoPE

Ann. Rep. U. S. Nat. Mus., 1898 (1900), p. 238, fig. 17.

TYPE LOCALITy: Cape St. Lucas, Lower California.

RANGE: Cape region of Lower California, north to San Esteban

Island.

\section{Ctenosaura multispinis CoPE}

Ctenosaura multispinis CoPE

Proc. Amer. Philos. Soc., Vol. 23, 1885, p. 267.

Ctenosaura multispinis Cope

Ann. Rep. U. S. Nat. Mus., 1898 (1900), p. 240.

TYPE LOCALITY: Dondomingvillo [sic] = Dondomuiguillo, Oaxaca and Batopilas Chihuahua.

RANGE: Mexico probably northward to southern Arizona.

\section{Dipso-saurus ( $G E N U S$ )}

Halloweld, Proc. Ac. Nat. Sci. Phila., Vol. 7, 1854, p. 92 TYPE: dorsalis

\section{Dipso-saurus carmenensis Van Denburgh}

Dipsosaurus carmenensis VAN Denburgh

Occ. Papers Calif. Ac. Sci., Vol. 10, Nov. 23, 1922, p. 81.

TyPE LOCALity: Carmen Island, Gulf of California.

RANGE: Carmen and Coronado Islands, Lower California.

Dipso-saurus catalinensis Van Denburgh

Dipsosaurus catalinensis VAN DeNBURGH

Oce. Papers Calif. Ac. Sci., Vol. 10, Nov. 23, 1922, p. 83.

TyPE LOCALITy: Santa Catalina Island, Gulf of California. RANGE: Same.

Dipso-saurus dorsalis dorsalis (Baird and GIRARD)

Crotaphytus dorsalis BAIRD and GIRARD

Proc. Ac. Nat. Sci. Phila., Vol. 6, 1852, p. 126. 
Dipso-saurus dorsalis HALLowell

Proc. Ac. Nat. Sci. Phila., Vol. 7, 1854, p. 92.

Dipsosaurus dorsalis Cope

Ann. Rep. U. S. Nat. Mus., 1898 (1900), p. 243, fig. 18.

TyPe locality: Colorado Desert, California.

RANGE: Colorado and Mojave Deserts, east to the Colorado River.

Dipso-saurus dorsalis lucasensis Van Denburgh

Dipsosaurus dorsalis lucasensis $\mathrm{V}_{\mathrm{AN}}$ DenbUrGH

Proc. Calif. Ac. Sci., Ser. 4, Vol. 10, Aug. 6, 1920, p. 33.

TyPe locality: San José del Cabo, Lower California.

RANGE: Cape region of Lower California.

\section{Crotaphytus ( $G E N U S$ )}

HoLвrook, N. Amer. Herp., Ed. 2, Vol. 2, 1842, p. 79

TYPE: collaris

Crotaphytus collaris collaris (SAY)

COLLARED LIZARD, MOUNTAIN BOOMER

Agama collaris $\mathrm{SAY}$

Long's Exp. Rocky Mts., Vol. 2, 1823, p. 252.

Crotaphytus collaris HolbrooK

N. Amer. Herp., Ed. 2, Vol. 2, 1842, p. 79, pl. 10.

Crotaphytus collaris (part) COPE

Ann. Rep. U. S. Nat. Mus., 1898 (1900), p. 248.

TyPE LOCALity: Verdigris River near its union with the Arkansas River, Oklahoma.

RANGE: Missouri, Kansas and Arkansas south to middle-western and northwestern Texas and west to eastern New Mexico.

Crotaphytus collaris baileyi (STEJNEGER)

Crotaphytus baileyi STEJNEGER

N. Amer. Fauna, No. 3, 1890, p. 103, pl. 12, fig. 1.

Crotaphytus collaris baileyi $\mathrm{STONE}$

Proc. Ac. Nat. Sci. Phila., 1903, p. 30.

Crotaphytus collaris (part) CoPE

Ann. Rep. U. S. Nat. Mus., 1898 (1900), p. 250, fig. 19.

ty

RANGE: Southwestern Texas, southern and western New Mexico,

Arizona, Utah, Nevada, Idaho, southeastern California, northern Mexico, and Lower California. 
Crotaphytus dickersonae ScHMidT

Crotaphytus dickersonae Sспмгот

Bull. Amer. Mus. Nat. Hist., Vol. 46, Dec. 7, 1922, p. 638, fig. 2.

TYPE LOCALITy: Tiburon Island, Gulf of California.

RANGE: Same.

Crotaphytus insularis VAN Denburgh and SLevin

Crotaphytus insularis Van Denburgh and SLevin

Proc. Calif. Ac. Sci., Ser 4, Vol. 11, July 30, 1921, p. 96.

type locality: Angel de la Guarda Island, Lower California.

RANGE: Same.

Crotaphytus reticulatus BAIRD

Crotaphytus reticulatus $\mathrm{BAIRD}_{\mathrm{A}}$

Proc. Ac. Nat. Sci. Phila., 1858, p. 253.

Crotaphytus reticulatus COPE

Ann. Rep. U. S. Nat. Mus., 1898 (1900), p. 254, fig. 20.

TyPE LOCAlity: Laredo and Ringgold Barracks, Texas.

RANGE: Extreme southwestern Texas.

Crotaphytus silus STEJNeger

Crotaphytus silus Stejneger

N. Amer. Fauna, No. 3, 1890, p. 105.

Crotaphytus wislizenii (part) COPE

Ann. Rep. U. S. Nat. Mus., 1898 (1900), p. 255.

TYPE LOCALITY: Fresno, California.

RANGE: San Joaquin Valley, California, Washington.

Crotaphytus wislizenii BaIRD and GIRARD

LEOPARD-LIZARD

Crolaphytus wislizenii BAIRD and GIRARD

Stansbury's Expl. Surv. Vall. Great Salt Lake, 1852, p. 340, pl. 3.

Crotaphytus wislizenii (part) COPE

Ann. Rep. U. S. Nat. Mus., 1898 (1900), p. 255, fig. 21.

ty ye Locality: Near Santa Fé, New Mexico.

RANGE: Northern Idaho, Nevada and Utah, southward into

Mexico, Lower and southern California; east to western Texas. 
Sauromalus ( $G E N U S$ )

Duméril, Arch. Mus. Hist. Nat. Paris, Vol. 8, 1856, p. 535

TYPE: ater

Sauromalus ater DUMÉRIL

CHUCK-WALLA

Sauromalus ater DUMÉRIL

Arch. Mus. Hist. Nat. Paris, Vol. 8, 1856, p. 536, pl. 23, fig. 3.

TYPE LOCALITY: Unknown.

RANGE: Southern Lower California and Espiritu Santo Island.

Sauromalus hispidus SteJNEger

Sauromalus hispidus STEJNEGER

Proc. U. S. Nat. Mus., Vol. 14, Oct. 27, 1891, p. 409.

Sauromalus hispidus Cope

Ann. Rep. U. S. Nat. Mus., 1898 (1900), p. 264, fig. 22.

TyPe LOCALity: Angel de la Guarda Island, Gulf of California. RANGE: Same as type locality.

Sauromalus obesus (BAIRD)

Euphryne obesus BAIRD

Proc. Ac. Nat. Sci. Phila., 1858, p. 253.

Sauromalus obesus Schmidt

Bull. Amer. Mus. Nat. Hist., Vol. 46, Dec. 7, 1922, p. 641.

Sauromalus ater Cope

Ann. Rep. U. S. Nat. Mus., 1898 (1900), p. 266, fig. 23.

TYPE LOCALITY: Fort Yuma, Arizona.

RANGE: Southern Nevada, southwestern Utah, Arizona, southern

California, and northern Lower California.

Sauromalus slevini VAN Denburgh

Sauromalus slevini $V_{\text {AN DENBURGH }}$

Occ. Papers Calif. Ac. Sci., Vol. 10, Nov. 23, 1922, p. 97.

TYPE LOCALITY: Monserrate Island, Gulf of California.

RANGE: Monserrate, Carmen, and Coronado Islands, Lower Cali-

fornia.

\section{Sauromalus townsendi Dickerson}

Sauromalus townsendi Dickerson

Bull. Amer. Mus. Nat. Hist., Vol. 41, Oct. 2, 1919, p. 464.

TYPE LOCALITY: Tiburon Island, Gulf of California.

RANGE: Tiburon Island and possibly adjacent coast region of

Sonora, Mexico. 
Sauromalus varius Dickerson

Sauromalus varius Dickenson

Bull. Amer. Mus. Nat. Hist., Vol. 41, Oct. 2, 1919, p. 464.

TYPE LOCALITY: San Esteban Island, Gulf of California. RANGE: Same.

\section{Callisaurus ( $G E N U S$ )}

Blainville, Nouv. Ann. Mus. Hist. Nat. Paris, Vol. 4, 1835, p. 286 TYPE: draconoides

\section{Callisaurus crinitus CoPE}

Callisaurus crinitus CoPE

Amer. Nat., Vol. 30, Dec. 5, 1896, p. 1049.

TyPE LOCALITY: Ballenas Bay, Lower California.

RANGE: Ballenas Bay and San Bartolomé Bay, Lower California.

Callisaurus draconoides BLAINviLLE

Callisaurus draconoides Brainville

Nouv. Ann. Mus. Hist. Nat. Paris, Vol. 4, 1835, p. 286, pl. 24, fig. 2. Callisaurus draconoides draconoides COPE

Ann. Rep. U. S. Nat. Mus., 1898 (1900), p. 270, fig. 24.

TYPE LOCALITY: California.

RANGE: Southern Lower California.

Callisaurus splendidus Dickerson

Callisaurus splendidus Dickerson

Bull. Amer. Mus. Nat. Hist., Vol. 41, Oct. 2, 1919, p. 467.

ty RANGE: Same.

Callisaurus ventralis ventralis (HALLOWELL) GRIDIRON-TAILED LIZARD

Homalosaurus ventralis HaLLowelL

Proc. Ac. Nat. Sci. Phila., Vol. 6, 1852, p. 179.

Callisaurus ventralis BAIRD

U. S. Mex. Bound. Surv., Vol. 1, Pt. 2, 1859, Rept., p. 8.

Callisaurus draconoides ventralis CoPE

Ann. Rep. U. S. Nat. Mus., 1898 (1900), p. 272, fig. 25.

TyPE LOCALity: New Mexico west of Rio Grande.

RANGE: Arizona to California, north to Nevada, southward to

Mexico at least to Guaymas. 


\title{
Callisaurus ventralis gabbii CoPE
}

Callisaurus ventralis gabbii COPE

Ann. Rep. U. S. Nat. Mus., 1898 (1900), p. 272.

TYPE LOCALITY: Northern Lower California.

RANGE: Lower California, deserts of southern California and adjacent deserts in Arizona.

Callisaurus ventralis inusitatus (DICKERson)

Callisaurus inusitatus Dickerson

Bull. Amer. Mus. Nat. Hist., Vol. 41, Oct. 2, 1919, p. 465.

TYPE LOCALITY: Tiburon Island, Gulf of California.

RANGE: Tiburon Island and region about Guaymas, Mexico.

Callisaurus ventralis myurus Richardson

Callisaurus ventralis myurus Richardson

Proc. U. S. Nat. Mus., Vol. 48, Jan. 19, 1915, p. 408.

TyPE LOCALITy: Pyramid Lake Indian Agency, Nevada.

RANGE: Region about Pyramid Lake, Nevada.

\author{
Uma ( $G E N U S)$ \\ BaIrd, Proc. Ac. Nat. Sci. Phila., 1858, p. 253 \\ TYPE: notata
}

\section{Uma notata BAIRD}

Uma notata BAIRD

Proc. Ac. Nat. Sci. Phila., 1858, p. 253.

Uma notata CoPE

Ann. Rep. U. S. Nat. Mus., 1898 (1900), p. 277, fig. 27.

TyPE LOCAlity: Mojave Desert, California.

RANGE: Yuma and Mojave Deserts.

\section{Holbrookia ( $G E N U S)$}

Girard, Proc. Amer. Assoc. Adv. Sci., New Haven, Vol. 4, 1851, p. 201 TYPE: maculata

\section{Holbrookia elegans BocourT}

Holbrookia elegans BocourT

Miss. Sci. Mex., Rept., 1874, p. 164, pl. 27 bis, figs. 8-8a.

TYPE LOCALity: Mazatlan, Mexico.

RANGE: Western Mexico from Sinaloa northward to Tucson,

Arizona. 
Holbrookia maculata maculata (GIRARD)

Holbroolia maculata GirARD

Proc. Amer. Assoc. Adv. Sci., New Haven, Vol. 4, 1851, p. 201.

IIolbrookia maculata maculata $\mathrm{COPE}$

Ann. Rep. U. S. Nat. Mus., 1898 (1900), p. 293, fig. 33.

TYPE L OCALITy: Opposite Grand Island, Platte River, Colorado.

RANGE: Northern Texas west to Arizona and northward to Wyoming and Nebraska.

Holbrookia maculata approximans (BAIRD)

IIolbroolia approximans BAIRD

Proc. Ac. Nat. Sci. Phila., 1858, p. 253.

Holbrookia maculata approximans STEJNEGER

N. Amer. Fauna, No. \&, 1890, p. 109.

Holbrookia maculata approximans CoPE

Ann. Rep. U. S. Nat. Mus., 1898 (1900), p. 297.

TyPE LOCALity: “Lower Rio Grande."

RANGE: Southern New Mexico and Arizona, south into northern Mexico.

\section{Holbrookia lacerata CoPE}

IIolbrookia lacerata Cope

Bull. U. S. Nat. Mus., No. 17, 1880, p. 15.

Holbroolia maculata lacerata STEJNEGER

N. Amcr. Fauna, No. 3, 1890, p. 109.

IIolbrookia maculata lacerata Cope

Ann. Rep. U. S. Nat. Mus., 1898 (1900), p. 292, fig. 32.

TYPE LOCALITY: Texas: Erath County; west of the Upper Brazos, Comanche County; on the Guadalupe River in Kendall or Comal County.

RANGE: Texas, middle district east of the plains and west of the timbered region, from the northern boundary south to the Rio Grande.

\section{Holbrookia propinqua propinqua (BAIRD and GIRARD)}

Holbrookia propinqua BAIRD and GIRARD

Proc. Ac. Nat. Sci. Phila., Vol. 6, 1852, p. 126.

IIolbrookia propinqua COPE

Ann. Rep. U. S. Nat. Mus., 1898 (1900), p. 289, fig. 31.

ту PE LOCality: Between Indianola and San Antonio, Texas.

RANGE: Texas. 
Holbrookia propinqua stonei HARPER

Holbrookia propinqua stonei $\mathrm{H}_{\mathrm{ARPER}}$

Proc. Biol. Soc. Wash., Vol. 45, Apr. 2, 1932, p. 15.

ty pe locality: Padre Island, Texas.

RANGe: Padre Island and possibly Mustang Island, Texas.

\section{Holbrookia pulchra ScHMID'T}

Holbrookia pulchra SснміDт

Amer. Mus. Nov., No. 22, Dec. 1, 1921, p. 1.

TyPE LOCAlity: Carr Cañon, Huachuca Mts., Arizona.

RANGE: Huachuca Mts., east of Nogales, Arizona.

\section{Holbrookia texana (Troschel)}

Cophosaurus texanus TroscheL

Arch. Naturg., Jahrg. 16, Vol. 1, 1850 (1852), p. 389, pl. 6.

Holbrookia texana BAIRD and GiraRD

Proc. Ac. Nat. Sci. Phila., Vol. 6, 1852, p. 124.

Holbrookia texana COPE

Ann. Rep. U. S. Nat. Mus., 1898 (1900), p. 286, fig. 30.

TYPE LOCALITY: The German colony of Neubraunfels, on the

Guadalupe River in western Texas, Lat. $28^{\circ} \mathrm{N}$.

RANGE: Arizona, Texas and eastern New Mexico.

\section{Uta ( $G E N U S)$ \\ BAIRD and Girard, Stansbury's Expl. Surv. Vall. \\ Great Salt Lake, 1852, p. 345 \\ TYPE: stansburiana}

\section{Uta auriculata Cope}

Uta auriculata CopE

Proc. Boston Soc. Nat. Hist., Vol. 14, 1871, p. 303.

Ty PE LOCALIty: Socorro Island, Revilla Gigedo Archipelago,

Mexico.

RANGE: The type locality.

Uta clarionensis Townsend

Uta clarionensis Townsend

Proc. U. S. Nat. Mus., Vol. 13, Sept. 9, 1890, p. 143.

Ty PE Locality: Clarion Island, Revilla Gigedo Archipelago, Mexico.

RANGE: Same as type locality. 
Uta concinna Dickerson

Uta concinna Dickerson

Bull. Amer. Mus. Nat. Hist., Vol. 41, Oct. 2, 1919, p. 470.

TYPE LOCALITY: Cerros Island, Lower California.

RANGE: Cerros and Natividad Islands, Lower California.

Uta graciosa (HALLOWELL)

Urosaurus graciosus HALLOWELL

Proc. Ac. Nat. Sci. Phila., Yol. 7, 1854, p. 92.

Uta graciosa BAlRD

U. S. Mex. Bound. Surv., Vol. 2, Pt. 2, 1859, Rept., p. 7.

Uta graciosa Cope

Ann. Rep. U. S. Nat. Mus., 1898 (1900), p. 325, fig. 46.

TYPE LOCALITY: "Lower California" - with the intention of saying southern California.

RANGE: Southern California, southwestern Arizona, southern

Nevada, and northeastern Lower California.

Uta levis Stejneger

Uta levis STEJNEGER

N. Amer. Fauna, No. 3, 1890, p. 108.

Uta levis Cope

Ann. Rep. U. S. Nat. Mus., 1898 (1900), p. 313, fig. 40.

Uta wrighti SCHMIDT

Amer. Mus. Nov., No. 22, Dec. 1, 1921, p. 3.

ty ye LOCAlity: Tierra Amarilla, New Mexico.

RANGE: New Mexico, southern Utah, and western Colorado.

\section{Uta mannophorus DiCKERsoN}

Uta mannophorus Dickerson

Bull. Amer. Mus. Nat. Hist., Vol. 41, Oct. 2, 1919, p. 470.

TYPE LOCALITY: Carmen Island, Gulf of California.

RANGE: Same.

Uta martinensis Van Denburgh

Uta martinensis VAN DENBURGI

Proc. Calif. Ac. Sci., Ser. 3, Zool., Vol. 4, 1905, p. 18, pl. 6. TYPE LOCA LITY: San Martin Island, Lower California. RANGE: San Martin Island.

\section{Uta mearnsi StEJNEGeR}

Uta mearnsi STEJNEGER

Proc. U. S. Nat. Mus., Vol. 17, Nov. 30, 1894, p. 589.

Uta mearnsii Cope

Ann. Rep. U. S. Nat. Mus., 1898 (1900), p. 304, fig. 37. 
TYPE LOCALITY: Summit of the Coast Range, Mexican boundary of California.

RANGE: Boundary region of southern and Lower California.

Uta microscutata Van Denburgh

Uta microscutata VAN DeNBURGH

Proc. Calif. Ac., Sci. Ser 2, Vol. 4, 189., p. 298.

Uta parviscutata CoPE

Ann. Rep. U. S. Nat. Mus., 1898 (1900), p. 324, fig. 45.

TyPe Locality: San Pedro Martir Mountains, Lower California.

RANGE: Extreme southern California, both east and west of the mountains, northern Lower California and San José Island, Lower California.

Uta nigricauda Cope

Uta nigricauda COPE

Proc. Ac. Nat. Sci. Phila., 1864, p. 176.

Uta nigricauda Cope

Ann. Rep. U. S. Nat. Mus., 1898 (1900), p. 322, fig. 44.

TyPE locality: Cape St. Lucas, Lower California.

RANGE: Southern Lower California.

Uta nolascensis VaN Denburgh and Slevin

Uta nolascensis VAN Denburgh and Slevin

Proc. Calif. Ac. Sci., Scr. 4, Vol. 11, Dec. 17, 1921, p. 395.

ty pe locality: San Pedro Nolasco Island, Lower California. RANGE: Same.

\section{Uta ornata ornata (BAIRD and GIRARD)}

Uta ornata BAIRD and GiraRD

Proc. Ac. Nat. Sci. Phila., Vol. 6, 1852, p. 126.

Uta ornata CopE

Ann. Rep. U. S. Nat. Mus., 1898 (1900), p. 315, fig. 41.

ty pe locality: Rio San Pedro, Texas, and Sonora.

RANGE: Utah, Nevada, western Texas west to southern California, northern Mexico.

Uta ornata symmetrica (BAIRD)

Uta symmetrica BAIRD

Proc. Ac. Nat. Sci. Phila., 1858, p. 253.

Uta symmetrica Cope

Ann. Rep. U. S. Nat. Mus., 1898 (1900), p. 317, fig. 42.

TYPE LOCALITY: Fort Yuma, Arizona.

RANGE: Colorado Desert and Colorado River Valley. 
Uta palmeri STEJNEGER

Uta palmeri STEJNEGER

N. Amer. Fauna, No. 3, 1890, p. 106.

Uta palmerii Cope

Ann. Rep. U. S. Nat. Mus., 1898 (1900), p. 313, fig. 39.

type locality: San Pedro Martir Island, Gulf of California. RANGE: Same.

Uta repens Van Denburgh

Uta repens $\mathrm{V}_{\text {AN }}$ Denburgh

Proc. Calif. Ac. Sci., Ser. 2, Vol. 5, 1895, p. 102, pl. 7-8, figs. a-e. Uta repens Cope

Ann. Rep. U. S. Nat. Mus., 1898 (1900), p. 303, fig. 36.

TYPE LOCALITY: Comondú, Lower California.

RANGE: Lower California.

Uta slevini Van Denburgh

Uta slevini Van Denburgh

Occ. Papers Calif. Ac. Sci., Vol. 10, Nov. 23, 1922, p. 194.

TYPE LOCALITY: Mejia Island, Gulf of California.

RANGE: Angel de la Guarda Island and Mejia Island, Lower

California.

Uta squamata Dickerson

Uta squamata Dickerson

Bull. Amer. Mus. Nat. Hist., Vol. 41, Oct. 2, 1919, p. 471.

TYPE LOCALITy: Santa Catalina Island, Gulf of California. RANGE: Same.

Uta stansburiana stansburiana (BAIRD and GIRARD)

Uta stansburiana BAIRD and GIRARD

Stansbury's Expl. Surv. Vall. Great Salt Lake, 1852, p. 345, pl. 5, figs. 4-6.

Uta stansburiana COPE

Ann. Rep. U. S. Nat. Mus., 1898 (1900), p. 306, fig. 38.

TYPE LOCALITY: Salt Lake Valley.

RANGE: Parts of Utah, Idaho, Nevada, and southern California.

Uta stansburiana elegans (YARROw)

Uta elegans YARRow

Proc. U. S. Nat. Mus., Vol. 5, Sept. 29, 1882, p. 442.

Uta stansburiana elegans RICHARDSON

Proc. U. S. Nat. Mus., Vol. 48, Jan. 19, 1915, p. 413.

ty pe locality: La Paz, Lower California.

RANGE: Southern Lower California. 


\section{Uta stansburiana hesperis RICHARDSON}

Uta stansburiana hesperis RICHARDSON

Proc. U. S. Nat. Mus., Vol. 48, Jan. 19, 1915, p. 415.

ty Pe locality: Arroyo Seco Cañon, near Pasadena, Los

Angeles County, California.

RANGE: Coast region of southern California.

\section{Uta stansburiana stejnegeri SснмiDT}

Uta stansburiana stejnegeri SснміDт

Amer. Mus. Nov., No. 15, 1921, p. 1.

TYPE LOCALity: Mouth of Dry Cañon, Alamogordo, Otero

County, New Mexico.

RANGe: Western Texas and northern Mexico through New

Mexico and Arizona to the Coast Range in California; northeastern Lower California; Angel de la Guarda Island.

\section{Uta stellata VAN Denburgh}

Uta stellata $\mathrm{V}_{\mathrm{AN}}$ Denburgh

Proc. Calif. Ac. Sci., Ser. 3, Zool., Vol. 4, 1905, p. 21, pl. 8. TYPE LOCALity: San Benito Island, Lower California. RANGE: The type locality.

\section{Uta thalassina CopE}

Uta thalassina Cope

Proc. Ac. Nat. Sci. Phila., 1863, p. 104.

Uta thalassina Cope

Ann. Rep. U. S. Nat. Mus., 1898 (1900), p. 301, fig. 35. TYPE LOCALity: Cape St. Lucas, Lower California. RANGE: Southern Lower California.

\section{Sator ( $G E N U S)$}

Dickerson, Bull. Amer. Mus. Nat. Hist., Vol. 41, Oct. 2, 1919, p. 468 TYPE: grandaevus

\section{Sator angustus Dickerson}

Sator angustus Dickerson

Bull. Amer. Mus. Nat. Hist., Vol. 41, Oct. 2, 1919, p. 469. TYPE LOCALITY: Santa Cruz Island, Gulf of California. RANGE: Same.

\section{Sator grandaevus Dickerson}

Sator grandaevus Dickerson

Bull. Amer. Mus. Nat. Hist., Vol. 41, Oct. 2, 1919, p. 469. TYPE LOCALITY: Cerralvo Island, Gulf of California. RANGE: Same. 


\section{Sceloporus ( $G E N U S$ )}

Wiegmann, Isis, 1828, p. 369

TYPE: torquatus

Sceloporus clarkii BAIRD and GIRARD

Sceloporus clarkii BAIRD and GIRARD

Proc. Ac. Nat. Sci. Phila., Vol. 6, 1852, p. 127.

Sceloporus clarkii (part) CoPE

Ann. Rep. U. S. Nat. Mus., 1898 (1900), p. 358, fig. 54.

TYPE LOCALITY: Sonora.

RANGE: New Mexico, Arizona, and northern Mexico.

Sceloporus consobrinus consobrinus (BAIRD and Girard)

Sceloporus consobrinus BAIRD and Girard

Marcy's Expl. Red River, 1853, Rept., p. 237, Zoöl., pl. 10, figs. 5-12. Sceloporus undulatus consobrinus COPE

Ann. Rep. U. S. Nat. Mus., 1898 (1900), p. 377, fig. 60.

Type Locality: Oklahoma, Roger Mills Co., Red River.

RANGE: The Dakotas, southward to Texas and westward to California.

\section{Sceloporus consobrinus garmani (Boulenger)}

Sceloporus garmani Boulenger

Proc. Zool. Soc. London, 1882, p. 761, pl. 56.

Sceloporus consobrinus garmani STEJNEGER and BARBouR

Check List N. Amer. Amph. Rept., Ed. 1, 1917, p. 53.

Ty pe Locality: Near Pine Ridge, South Dakota.

RANGE: Region about type locality.

\section{Sceloporus couchii BAIRD}

Sceloporus couchii BAIRD

Proc. Ac. Nat. Sci. Phila., 1858, p. 254.

Lysoptychus lateralis COPE

Ann. Rep. U. S. Nat. Mus., 1898 (1900), p. 329, fig. 47.

Sceloporus couchii Cope

Ann. Rep. U. S. Nat. Mus., 1898 (1900), p. 395, fig. 66.

Ty PE LoCAlity: Santa Caterina, Nuevo Leon, Mexico.

RANGE: Northern Mexico to southern Texas.

Sceloporus disparilis STEJNEgER

Sccloporus disparilis STEJNEGER

Proc. Biol. Soc. Washington, Vol. 29, Dec. 16, 1916, p. 228. 
TYPE LOCALITY: Lomita Ranch, six miles north of Hidalgo, Texas.

RANGE: Extreme southern corner of Texas and northeastern Mexico.

\section{Sceloporus elongatus STEJNEGER}

Sceloporus elongatus STEJNEGER

N. Amer. Fauna, No. 3, 1890, p. 111.

Sceloporus undulatus (part) COPE

Ann. Rep. U. S. Nat. Mus., 1898 (1900), p. 368.

ty pe Locality: Moa Ave, Painted Desert, Arizona.

RANGE: Northern Arizona and southern Utah.

\section{Sceloporus graciosus graciosus (BAIRD and GIRARD)}

Sceloporus graciosus BAIRD and GIRARD

Stansbury's Expl. Surv. Vall. Great Salt Lake, 1852, p. 346, pl. 5, figs. 1-3.

Sceloporus graciosus graciosus CAMP

Univ. California Publ. Zool., Vol. 17, Dec. 28, 1916, p. 67.

Sceloporus graciosus (part) CoPE

Ann. Rep. U. S. Nat. Mus., 1898 (1900), p. 386.

TYPE LOCALITY: Valley of Great Salt Lake.

RANGE: Oklahoma to California and Utah, Nevada.

\section{Sceloporus graciosus gracilis (BAIRD and GIRARD)}

Sceloporus gracilis BAIRD and GIRARD

Proc. Ac. Nat. Sci. Phila., Vol. 6, 1852, p. 175.

Sceloporus graciosus gracilis VAN DenBURGH

Occ. Papers Calif. Ac. Sci., Vol. 10, Nov. 23, 1922, p. 280.

Sceloporus graciosus (part) CopE

Ann. Rep. U. S. Nat. Mus., 1898 (1900), p. 386, fig. 63.

TYPE LOCALITY: Oregon.

RANGE: Western Oregon and Washington and northern California.

\section{Sceloporus graciosus vandenburgianus (COPE)}

Sceloporus vandenburgianus CopE

Amer. Nat., Vol. 30, 1896, p. 834.

Sceloporus graciosus vandenburgianus CAMP

Univ. California Publ. Zool., Vol. 17, Dec. 28, 1916, p. 67.

Sceloporus vandenburgianus CopE

Ann. Rep. U. S. Nat. Mus., 1898 (1900), p. 390, fig. 64. 
TYPE LOcality: Summit of the Coast Range, San Diego County, California.

RANGE: Mountain ranges south of Ventura County, California, into northern Lower California.

Sceloporus jarrovii COPE

Sceloporus jarrovii CopE

in Wheeler's Rep. Surv. W. 100th Mer., Vol. 5, 1875, p. 569, pl. 23, figs. 2-2c.

Sceloporus jarrovii Cope

Ann. Rep. U. S. Nat. Mus., 1898 (1900), p. 345, fig. 49.

tyPE LOCALity: Southern Arizona.

RANGE: Arizona.

Sceloporus licki Van DenbURGH

Sceloporus licki VAN DenBURGH

Proc. Ac. Sci., Ser. 2, Vol. 5, 1895, p. 110, pl. 10.

Sceloporus lickii Cope

Ann. Rep. U. S. Nat. Mus., 1898 (1900), p. 363.

TYPE LOCALITY: Sierra San Lazaro, Lower California.

RANGE: Southern Lower California.

\section{Sceloporus lineatulus Dickerson}

Sceloporus lineatulus Dickerson

Bull. Amer. Mus. Nat. Hist., Vol. 41, Oct. 2, 1919, p. 467.

TyPe locality: Santa Catalina Island, Gulf of California. RANGE: Same.

\section{Sceloporus magister HaLlowell}

Sceloporus magister HaLlowell

Proc. Ac. Nat. Sci. Phila., 1854, p. 93.

Sceloporus clarkii (part) CoPE

Ann. Rep. U. S. Nat. Mus., 1898 (1900), p. 358.

TyPe locality: Yuma, Arizona.

RANGE: Southeastern California, western Arizona, southern

Nevada, southern and eastern Utah, and northwestern Lower

California.

\section{Sceloporus merriami Stejneger}

Sceloporus merriami STEJNEGER

Proc. Biol. Soc. Washingtou, Vol. 17, Feb. 5, 1904, p. 17.

ty pe Locality: East Painted Cave near mouth of Pecos River,

Rio Grande Valley, Texas.

RANGE: Rio Grande Cañon, western Texas. 
Sceloporus monserratensis Van Denburgh and Slevin

Sceloporus monserratensis VAN Denburgh and Slevin

Proc. Calif. Ac. Sci., Ser. 4, Vol. 11, Dec. 17, 1921, p. 396.

TYPE LOCALITy: Monserrate Island, Lower California.

RANGE: Same.

Sceloporus occidentalis occidentalis (BAIRD and GIRARD)

Sceloporus occidentalis BAIRD and GIRARD

Proc. Ac. Nat. Sci. Phila., Vol. 6, 1852, p. 175.

Sceloporus occidentalis occidentalis $\mathrm{C}_{\mathrm{AMP}}$

Univ. California Publ. Zool., Vol. 17, Dec. 28, 1916, p. 65.

TyPE LOCALITy: Benicia, California.

RANGE: Northern and north central California to Washington.

Sceloporus occidentalis becki (VAN Denburgh)

Sceloporus becki $\mathrm{V}_{\text {AN }}$ Denburgh

Proc. Calif. Ac. Sci., Ser. 3, Zool., Vol. 4, June 15, 1905, p. 9, pl. 4.

Sceloporus occidentolis becki GRINNELL and CAMP

Univ. California Publ. Zool., Vol. 17, July 11, 1917, p. 162.

type Locality: San Miguel Island, Santa Barbara Co., California.

RANGE: Islands off coast of Santa Barbara County, California.

Sceloporus occidentalis bi-seriatus (HALLOWELL)

Sceloporus bi-seriatus HaLlowell

Proc. Ac. Nat. Sci. Phila., Vol. 7, 1854, p. 93.

Sceloporus occidentalis bi-seriatus $\mathrm{CAMP}_{\mathrm{P}}$

Univ. California Publ. Zool., Vol. 17, Dec. 28, 1916, p. 65.

Sceloporus biseriatus Cope

Ann. Rep. U. S. Nat. Mus., 1898 (1900), p. 381, fig. 61.

TyPe LoCality: El Paso Creek, Tejon Valley, California.

RANGE: Texas westward to California, also Utah, Idaho, Wyoming, Nevada, Oregon and northern Lower California.

\section{Sceloporus occidentalis taylori CAMP}

Sceloporus occidentalis taylori $\mathrm{CAMP}$

Univ. California Publ. Zool., Vol. 17, Dec. 28, 1916, p. 65.

TYPE LOCALITY: Halfway between Merced Lake and Sunrise Trail (Echo Creek Basin), altitude 7500 feet, Yosemite National Park, California.

RANGE: Yosemite National Park, above 7000 feet altitude. 


\section{Sceloporus orcutti STEJNEGER}

Sceloporus orcutti STEJNEGER

N. Amer. Fauna, No. 7, May 31, 1893, p. 181, pl. 1, figs. 4a-c.

Sceloporus orcuttii Cope

Ann. Rep. U. S. Nat. Mus., 1898 (1900), p. 354, fig. 52.

Ty PE LoCality: Milquatay Valley, San Diego Co., California.

RANGE: Northern Lower California, southern California.

Sceloporus rufidorsum YARROW

Sceloporus rufidorsum (part) YARROW

Proc. U. S. Nat. Mus., Vol. 5, 1882, p. 442.

TYPE LOCALITY: San Quentin Bay, [Lower] California.

RANGE: Western Lower California and Cerros Island.

\section{Sceloporus scalaris WiegmanN}

Sceloporus scalaris WiegmanN

Isis, 1828, p. 370.

Sceloporus scalaris COPE

Ann. Rep. U. S. Nat. Mus., 1898 (1900), p. 401, fig. 68.

TYPE LOCALITY: Mexico.

RANGE: Mexico northward to the United States boundary.

Sceloporus spinosus floridanus (BAIRD)

Sceloporus floridanus BAIRD

Proc. Ac. Nat. Sci. Phila., 1858, p. 254.

Sceloporus spinosus floridanus STEJNEGER

N. Amer. Fauna, No. 7, May 31, 1893, p. 181.

Sceloporus spinosus (part) CoPE

Ann. Rep. U. S. Nat. Mus., 1898 (1900), p. 364, fig. 55.

TyPe Locality: Pensacola, Florida.

RANGE: Extreme western Florida to Texas, New Mexico, and northern Mexico.

\section{Sceloporus torquatus cyanogenys CoPE}

Sceloporus torquatus cyanogenys CoPE

Proc. Amer. Philos. Soc., Vol. 22, 1885, p. 402.

Sceloporus torquatus cyanogenys TAYLOR

Proc. Biol. Soc. Wash., Vol. 44, Oct. 17, 1931, p. 129.

TYPE LoCALity: Monterey, Nuevo Leon, Mexico.

RANGE: Northeastern Mexico and southeastern Texas along the lower 250 miles of the Rio Grande. 
Sceloporus torquatus poinsettii (BAIRD and GIRARD)

Sceloporus poinsettii BAIRD and GIRARD

Proc. Ac. Nat. Sci. Phila., Vol. 6, 1852, p. 126.

Sceloporus torquatus poinsettii $\mathrm{COPE}$

Proc. Amer. Philos. Soc., Vol. 22, 1885, p. 402.

Sceloporus torquatus poinsettii COPE

Ann. Rep. U. S. Nat. Mus., 1898 (1900), p. 350, fig. 51.

ty pe locality: Rio San Pedro, Texas, and Sonora, Mexico.

RANGE: Texas to Arizona, northern states of Mexico.

Sceloporus undulatus (LATREILLE)

PINE-LIZARD, FENCE-LIZARD

Stellio undulatus LATreille

Hist. Nat. Rept., Vol. 2, 1802, p. 40.

Sceloporus undulatus WIEGMANN

Isis, 1828, p. 369.

Sceloporus undulatus (part) CoPE

Ann. Rep. U. S. Nat. Mus., 1898 (1900), p. 368, fig. 57.

TYPE LOCALITY: "Les grands bois de la Caroline."

RANGE: Eastern States, southeastern New York to Florida.

Sceloporus variabilis WiegmanN

Sceloporus variabilis WiEgmanN

Herp. Mexico, 1834, p. 51.

Sceloporus variabilis COPE

Ann. Rep. U. S. Nat. Mus., 1898 (1900), p. 398, fig. 67.

TYPE LOCALITY: Mexico.

RANGE: Guatemala and Mexico to southern Texas.

Sceloporus woodi STEJNEGER

SOUTHERN FLORIDA PINE-LIZARD

Sceloporus woodi STEJNEGER

Proc. Biol. Soc. Washington, Vol. 31, June 29, 1918, p. 90.

TYPE locality: Auburndale, Polk Co., Florida.

RANGE: Spruce Pine ridges of central, southeast and extreme southwest Florida.

\section{Sceloporus zosteromus CoPE}

Sceloporus zosteromus CopE

Proc. Ac. Nat. Sci. Phila., 1863, p. 105.

Sceloporus zosteromus Cope

Ann. Rep. U. S. Nat. Mus., 1898 (1900), p. 356, fig. 53.

TYPE LOCALITY: Cape St. Lucas, Lower California.

RANGE: Southern and central Lower California. 


\section{Phrynosoma ( GENUS) \\ WIEGMANN, Isis, 1828, p. 367 \\ TYPE: orbiculare}

THE HORN-TOADS

Phrynosoma blainvillii blainvillii (GRAY)

Phrynosoma blainvillii GraY

Zoöl. Beechey's Voyage, 1839, p. 96, pl. 29, fig. 1.

Phrynosoma blainvillei blainvillei Bryant

Univ. California Publ. Zool., Vol. 9, 1911, pp. 5, 29.

Phrynosoma blainvillei (part) CoPE

Ann. Rep. U. S. Nat. Mus., 1898 (1900), p. 423, fig. 74.

TYPE LOCALITY: California.

RANGE: Southern California, northern Lower California.

\section{Phrynosoma blainvillii frontale (VAN Denburgh)}

Phrynosoma frontalis VAN Denburgh

Proc. Calif. Ac. Sci., Ser. 2, Vol. 4, 1894, p. 296.

Phrynosoma blainvillei (part) CoPE

Ann. Rep. U. S. Nat. Mus., 1898 (1900), p. 423.

Phrynosoma frontale Stejneger and Barbour

Check List N. Amer. Amph. Rept., Ed. 1, 1917, p. 59.

тy Pe Locality: Bear Valley, San Benito County, California. RANGe: El Dorado to Kern Counties, California.

\section{Phrynosoma brevirostre (GIRARD)}

Tapaya brevirostris GIRARD

U. S. Expl. Exp., Herp., 1858, p. 397.

Phrynosoma brevirostre CoPE

Proc. Ac. Nat. Sci. Phila., 1866, p. 302.

TYPE LOCALITY: Plains of Nebraska.

RANGE: North Dakota, Wyoming, the basins of the Yellowstone and Platte Rivers.

\section{Phrynosoma cerroense Stejneger}

Phrynosoma cerroense Stejneger

N. Amer. Fauna, No. 7, May 31, 1893, p. 187.

Phrynosoma cerroense Cope

Ann. Rep. U. S. Nat. Mus., 1898 (1900), p. 428, fig. 75.

Ty PE LOCALITY: Cerros Island, Lower California.

RANGE: The type locality. 


\section{Phrynosoma cornutum (HARLAN)}

Agama cornuta Harlan

Journ. Ac. Nat. Sci. Phila., Vol. 4, 1825, p. 299, pl. 20.

Phrynosoma cornutum GRAY

Griffith's Animal Kingdom, Syn. Rept., 1831, p. 9.

Phrynosoma brevicorne Stejneger and Barbour

Check List N. Amer. Amph. Rept., Ed. 2, 1923, p. 60.

Phrynosoma cornutum CoPE

Ann. Rep. U. S. Nat. Mus., 1898 (1900), p. 432, fig. 77.

TYPE LOCALITY: Great Plains east of the Rocky Mountains.

RANGE: Arkansas, Kansas to the northern states of Mexico, west

to Colorado, New Mexico, and Arizona.

\section{Phrynosoma coronatum (BLAinville)}

Agama (Phrynosoma) coronata Blainville

Nouv. Ann. Mus. Hist. Nat. Paris, Vol. 4, 1835, p. 284, pl. 25, fig. 1.

Phrynosoma coronatum Duméril and Bibron

Erp. Gén., Vol. 4, 1837, p. 318.

Phrynosoma coronatum CoPE

Ann. Rep. U. S. Nat. Mus., 1898 (1900), p. 430.

TYPE LOCALITY: "California" by inference.

RANGE: Southern Lower California.

\section{Phrynosoma ditmarsi Stejneger}

Phrynosoma ditmarsi SteJNeger

Proc. U. S. Nat. Mus., Vol. 29, Feb. 2, 1906, p. 565.

TYPE LOCALity: State of Sonora, Mexico, not far from boundary of Arizona.

RANGE: Northern Mexico and probably southern Arizona.

Phrynosoma douglassii douglassii (BELL)

Agama douglassii BeLL

Trans. Linn. Soc. London, Vol. 16, 1833, p. 105, pl. 10.

Phrynosoma douglassii douglassii COPE

Ann. Rep. U. S. Nat. Mus., 1898 (1900), p. 411, fig. 69.

TYPE LOCALITY: "In ora occidentali Americae Borealis ad ripas fluminis Columbiae."

RANGE: Oregon and Washington.

\section{Phrynosoma douglassii hernandesi (GIRARd)}

Tapaya hernandesi GiRARD

U. S. Expl. Exp., Herp., 1858, p. 395.

Phrynosoma douglassii hernandesi CoPE

Ann. Rep. U. S. Nat. Mus., 1898 (1900), p. 413, fig. 70. 
TyPe LOCAlity: New Mexico.

RANGE: The plateau region of Colorado, southern New Mexico, southeastern and central Arizona, southwestern Texas, and northern Sonora.

Phrynosoma douglassii ornatissimum (Girard)

Tapaya ornatissima GIRARD

U. S. Expl. Exp., Herp., 1858, p. 396.

Phrynosoma douglassii ornatissimum COPE

Ann. Rep. U. S. Nat. Mus., 1898 (1900), p. 415, fig. 71.

TyPE LOCALITy: Mountainous region of New Mexico.

RANGE: Deserts of northeastern Arizona, northern New Mexico,

Colorado, Idaho, Nevada, and Utah.

Phrynosoma douglassii ornatum (GIRARD)

Phrynosoma ornatum GIRARD

U. S. Expl. Exp., Herp., 1858, Atlas, pl. 21, figs. 1-5.

Phrynosoma douglassii ornatum STEJNEGER

Copeia, No. 65, 1919, p. 4.

TYPE LOCALITY: Salt Lake.

RANGE: The Salt Lake basin.

Phrynosoma jamesi Schmidt

Phrynosoma jamesi Schuidt

Bull. Amer. Mus. Nat. Hist., Vol. 46, Dec. 7, 1922, p. 668, pl. 55-56. TYPE LOCALity: San Bartolomé Bay, Lower California. RANGE: Same.

\section{Phrynosoma m'callii (Halloweld)}

Anota m'callii Hallowell

Proc. Ac. Nat. Sci. Phila., Vol. 6, 1852, p. 182.

Phrynosoma maccallii CopE

Proc. Ac. Nat. Sci. Phila., 1866, p. 310.

Anota maccallii COPE

Ann. Rep. U. S. Nat. Mus., 1898 (1900), p. 448, fig. 82.

TYPE LOCALITY: Colorado Desert between Vallecita [sic] and

Camp Yuma, 160 miles east of San Diego, California.

RANGE: Southeastern California and southwestern Arizona.

\section{Phrynosoma modestum Girard}

Phrynosoma modestum GIRARD

Stansbury's Expl. Surv. Vall. Great Salt Lake, 1852, pp. 361, 365, pl. 6, figs. 4-8.

Anota modesta Cope

Ann. Rep. U. S. Nat. Mus., 1898 (1900), p. 437, fig. 78. 
TyPe locality: The Rio Grande, west of San Antonio, Texas, and from between San Antonio and El Paso.

RANG E: Texas to Arizona and northern Mexico.

\section{Phrynosoma nelsoni SchmidT}

Phrynosoma nelsoni Schmidt

Bull. Amer. Mus. Nat. Hist., Vol. 46, Dec. 7, 1922, p. 666, pl. 54, fig. 2. TyPE LOCALity: San Quentin, Lower California.

RANGE: Same.

\section{Phrynosoma platyrhinos GIRARD}

Phrynosoma platyrhinos Girard

Stansbury's Expl. Surv. Vall. Great Salt Lake, 1852, pp. 361, 363, pl. 7, figs. 1-5.

Anota platyrhina Cope

Ann. Rep. U. S. Nat. Mus., 1898 (1900), p. 443, fig. 81.

Ty PE LOCALITy: Great Salt Lake Valley, Utah.

RANGE: Washington southward to California, Utah, Idaho and

Nevada, southwestern Arizona and northeastern Lower California.

Phrynosoma schmidti Barbour

Phrynosoma schmidti BarBour

Proc. N. Engl. Zoöl. Club, Vol. 7, Sept. 7, 1921, p. 113.

TyPE LOCALity: Cerros Island, Lower California.

RANGE: Same.

Phrynosoma solare GraY

Phrynosoma solaris GRAX

Cat. Liz. Brit. Mus., 1845, p. 229.

Phrynosoma solare Cope

Ann. Rep. U. S. Nat. Mus., 1898 (1900), p. 420, fig. 73.

TYPE LOCALITY: "California."

RANGE: Northern Lower California and extreme southern Arizona and Sonora.

\section{ANGUIDAE (FAMILY) \\ Ophisaurus ( $G E N U S$ )}

Daudin, Bull. Soc. Philom. Paris, Vol. 3, No. 72, March, 1803, p. 188 TYPE: ventralis

Ophisaurus ventralis (LINNÉ)

GLASS-SNAKE, JOINT-SNAKE

Anguis ventralis LiNNé

Syst. Nat., Ed. 12, Vol. 1, 1766, p. 391. 
Ophisaurus ventralis DAUDIN

Hist. Nat. Rept., Vol. 7, 1803, p. 352, pl. 88.

Ophisaurus ventralis COPE

Ann. Rep. U. S. Nat. Mus., 1898 (1900), p. 494, fig. 88.

TYPE LOCALITY: Carolina.

RANGE: Southern United States, in the east distributed northward

to Virginia and Tennessee, in the central valley to Wisconsin, the dune region of northern Indiana, westward to New Mexico, and southward to the State of Vera Cruz, Mexico.

\section{Gerrhonotus ( $G E N U S$ )}

Wiegmann, Isis, 1828 , p. 379

TYPE: tessellatus = liocephalus

\section{Gerrhonotus coeruleus WIEGManN}

Gerrhonotus coeruleus Wiegainn

Isis, 1828, p. 380.

Gerrhonotus burnettii COPE

Ann. Rep. U. S. Nat. Mus., 1898 (1900), p. 526, fig. 94.

TYPE LOCALITY: "Brasilia" (in errore; probably San Francisco, Calif.).

RANGE: Coast region of northern California.

\section{Gerrhonotus infernalis BAIRD}

Gerrhonotus infernalis BAIRD

Proc. Ac. Nat. Sci. Phila., 1858, p. 255.

Gerrhonotus liocephalus infernalis CopE

Ann. Rep. U. S. Nat. Mus., 1898 (1900), p. 517, fig. 91.

TYPE LOCALity: Devil's River, Texas.

RANGE: Southern Texas and northern Mexico.

\section{Gerrhonotus kingii GraY}

Gerrhonotus kingii GRAY

Ann. Mag. Nat. Hist., Vol. 1, 1838, p. 390.

Gerrhonotus kingii VAN DenBurgh

Rept. of Western N. Amer., Vol. 1, 1922, p. 461.

Gerrhonotus nobilis STEJNEGER and BARBOUR

Check List N. Amer. Amph. Rept., Ed. 2, 1923, p. 65.

TYPE LOCALITY: Unknown.

RANGE: New Mexico, Arizona and northern Mexico.

Gerrhonotus multi-carinatus (BLAINville)

Cordylus (Gerrhonotus) multi-carinatus B LAINville

Nouv. Ann. Mus. Hist. Nat. Paris, Vol. 4, 1835, p. 289, pl. 25, fig. 2. 
Gerrhonotus multicarinatus DUMÉRIL and Bibron

Erp. Gén., Vol. 5, 1839, p. 404.

Gerrhonotus multicarinatus (part) COPE

Ann. Rep. U. S. Nat. Mus., 1898 (1900), p. 520.

TYPE LOCALITY: "California."

RANGE: Lower California.

\section{Gerrhonotus palmeri (STEJNEGER)}

Gerrhonotus scincicauda palmeri STEJNEGER

N. Amer. Fauna, No. 7, May 31, 1893, p. 196.

Gerrhonotus palmeri VAN DENBURGH

Occ. Papers Calif. Ac. Sci., No. 5, 1897, p. 113.

Gerrhonotus multicarinatus palmerii Cope

Ann. Rep. U. S. Nat. Mus., 1898 (1900), p. 525.

TYPE LOCALity: South Fork King's River, California.

RANGE: Sierra Nevada slopes, in central California.

\section{Gerrhonotus principis (BAIRD and GIRARD)}

Elgaria principis BAIRD and GIRARD

Proc. Ac. Nat. Sci. Phila., Vol. 6, 1852, p. 175.

Gerrhonotus principis SteJNeger

N. Amer. Fauna, No. 7, May 31, 1893, p. 197.

Gerrhonotus principis Cope

Ann. Rep. U. S. Nat. Mus., 1898 (1900), p. 529, fig. 95.

ty Pe Locality: Oregon and Puget Sound.

RANGE: British Columbia, western Washington and Oregon, northwestern California and Vancouver Island.

\section{Gerrhonotus scincicauda scincicauda (SKILTON)}

Tropidolepis scincicauda SkILTON

Amer. Journ. Sci. Arts, Ser. 2, Vol. 7, 1849, p. 202, pl. at p. 312, figs. 1-3.

Gerrhonotus scincicauda scincicauda Grinnell and CAMP

Univ. California Publ. Zool., Vol. 17, No. 10, 1917, p. 166.

Gerrhonotus multicarinatus (part) CoPE

Ann. Rep. U. S. Nat. Mus., 1898 (1900), p. 520, fig. 93.

TYPE LOCALITY: "Dalles of the Columbia."

RANGE: Washington, Oregon, and California west of the Sierra

Nevada.

\section{Gerrhonotus scincicauda webbii (BAIRD)}

Gerrhonotus webbii BAIRD

Proc. Ac. Nat. Sci. Phila., 1858, p. 255.

Gerrhonotus scincicauda webbii GRINNELL and CAMP

Univ. California Publ. Zool., Vol. 17, No. 10, 1917, p. 168. 
TYPE Locality: "From San Diego to El Paso." 1 RANGE: Southern California and northwestern Lower California.

Barissia ( $G E N U S)$

GraY, Cat. Liz. Brit. Mus., 1845, p. 54 TYPE: imbricata

Barissia levicollis STEJNEGER

Barissia lcvicollis STEJNEGER

Proc. U. S. Nat. Mus., Vol. 13, Sept. 9, 1890, p. 184.

Barissia levicollis COPE

Ann. Rep. U. S. Nat. Mus., 1898 (1900), p. 535, fig. 96.

TYPE LOCALITY: "Mexican Boundary."

RANGE: State of Durango, Mexico, north to the border.

ANNIELLIDAE (FAMILY)

Anniella ( $G E N U S$ )

GrAY, Ann. Mag. Nat. Hist., Ser. 2, Vol. 10, 1852, p. 440 TY PE: pulchra

Anniella nigra Fischer

Anniella nigra Fischer

Abh. Naturw. Ver. Hamburg, Vol. 9, Pt. 1, 1886, p. 9.

Anniella pulchra var. A. nigra Cope

Ann. Rep. U. S. Nat. Mus., 1898 (1900), p. 675.

TYPE LOCALit Y: San Diego, California, probably in errore.

RANGE: Country about Pacific Grove, California.

Anniella pulchra Gray

Anniella pulchra Gray

Ann. Mag. Nat. Hist., Ser. 2, Vol. 10, 1852, p. 440.

Anniella pulchra COPE

Ann. Rep. U. S. Nat. Mus., 1898 (1900), p. 674, fig. 138.

TYPE LOCALITY: California.

RANGE: Eastern and western slopes of the Sierras, coastal region of southern California and northern Lower California.

1 Baird's MS. entry in U.S.N.M. register. Type U.S.N.M. No. 3078, a very young individual. 


\title{
HELODERMATIDAE ( $F A M I L Y)$ \\ Heloderma ( $G E N U S)$ \\ WiegmanN, Isis, 1829, p. 624 \\ TYPE: horridum
}

\section{Heloderma suspectum CoPE}

GILA MONSTER

Heloderma suspectum Cope

Proc. Ac. Nat. Sci. Phila., 1869, p. 5.

Heloderma suspectum Cope

Ann. Rep. U. S. Nat. Mus., 1898 (1900), p. 476, fig. 87.

TYPE LOCALITY: Sierra de la Union, Arizona ("Sonora").

RANGE: Extreme southern Utah and Nevada, Arizona, and So-

nora.

\author{
XANTUSIIDAE (FAMILY) \\ Xantusia ( $G E N U S$ ) \\ BAIrd, Proc. Ac. Nat. Sci. Phila., 1858, p. 255 \\ TYPE: vigilis
}

Xantusia arizonae KLAUBER

Xantusia arizonae KLAUBER

Trans. San Diego Soc. Nat. Hist., Vol. 7, No. 1, Oct. 6, 1931, p. 1, pl. 1. TYPE LoCality: Yarnell, Yavapai County, Arizona.

RANGE: The type locality.

Xantusia gilberti VAN Denburgh

$X$ antusia gilberti $\mathrm{V}_{\mathrm{AN}}$ DenbURGH

Proc. Calif. Ac. Sci., Ser. 2, Vol. 5, 1895, p. 121, pl. 11.

Amoebopsis gilbertii CoPE

Ann. Rep. U. S. Nat. Mus., 1898 (1900), p. 555, fig. 101.

TYPE LOCALITY: San Francisquito, Sierra Laguna, Lower California.

RANGE: Southern Lower California.

\section{Xantusia henshawi Stejneger}

Xantusia henshawi STEJNEGER

Proc. U. S. Nat. Mus., Vol. 16, July 21, 1893, p. 467.

Zablepsis henshavii COPE

Ann. Rep. U. S. Nat. Mus., 1898 (1900), p. 553, fig. 100.

TYPE LOCALITY: Witch Creek, San Diego County, California.

RANGE: Rocky areas on both slopes of the mountains from south-

ern Riverside County, California to the San Pedro Martir

Mountains, Lower California. 
Xantusia riversiana Cope

Xantusia riversiana COPE

Proc. Ac. Nat. Sci. Phila., 1883, p. 29.

Xantusia riversiana $\mathrm{COPE}$

Ann. Rep. U. S. Nat. Mus., 1898 (1900), p. 550, fig. 99.

TYPE LOCALITY: California, according to Cope, but later stated

by Professor Rivers to have been San Nicolas Island, off the California coast.

RANGE: San Nicolas, Santa Barbara, and San Clemente Islands, coast of California.

Xantusia vigilis BAIRD

Xantusia vigilis BAIRD

Proc. Ac. Nat. Sci. Phila., 1858, p. 255.

Xantusia vigilis CoPE

Ann. Rep. U. S. Nat. Mus., 1898 (1900), p. 545, fig. 97.

TYPE LOCALIty: Fort Tejon, California.

RANGE: The deserts and desert mountains of the Californias and southern Nevada from the Inyo Mountains, Inyo County, California to San Felipe Bay and San Matias Pass, Lower California.

\author{
TEIIDAE $(F A M I L Y)$ \\ Cnemidophorus ( $G$ EN US ) \\ WAgler, Syst. Amph., 1830, p. 154 \\ TYPE: murinus \\ THE SWIFTS OR WHIP-TAILS
}

Cnemidophorus bacatus Van Denburgh and Stevin

Cnemidophorus bacatus Van Denburgh and Slevin

Proc. Calif. Ac. Sci., Ser. 4, Vol. 11, July 30, 1921, p. 97.

ty ye Locality: San Pedro Nolasco Island, Lower California.

RANGE: Same.

Cnemidophorus bartolomas Dickerson

Cnemidophorus bartolomas Dickerson

Bull. Amer. Mus. Nat. Hist., Vol. 41, Oct. 2, 1919, p. 476.

TYPE LOCALITY: San Bartolomé Bay, Lower California.

RANGE: Same. 
Cnemidophorus catalinensis Van Denburgh and Slevin

Cnemidophorus catalinensis VAN Denburgh and Slevin

Proc. Calif. Ac. Sci., Ser. 4, Vol. 11, Dec. 17, 1921, p. 396.

TYPE LOCALITY: Santa Catalina Island, Lower California.

RANGE: Same.

Cnemidophorus ceralbensis (Van Denburgh and Slevin)

Verticaria ceralbensis VAN Denburgh and Slevin

Proc. Calif. Ac. Sci., Ser. 4, Vol. 11, Dec. 17, 1921, p. 396.

TYPE LOCALity: Ceralbo Island, Lower California.

RANGE: Same.

Cnemidophorus estebanensis Dickerson

Cnemidophorus estebanensis Dickerson

Bull. Amer. Mus. Nat. Hist., Vol. 41, Oct. 2, 1919, p. 474.

TYPE LOCALity: San Esteban Island, Gulf of California.

RANGE: Same.

Cnemidophorus grahamii BaIRD and GIRARD

Cnemidophorus grahamii BaIrD and Girard

Proc. Ac. Nat. Sci. Phila., Vol. 6, 1852, p. 128.

Cnemidophorus grahamii grahamii CoPE

Ann. Rep. U. S. Nat. Mus., 1898 (1900), p. 598, fig. 117.

ty pe Locality: Between San Antonio and El Paso, Texas.

RANGE: Texas.

Cnemidophorus gularis BAIRd and Girard

Cnemidophorus gularis BAIRD and GIRARD

Proc. Ac. Nat. Sci. Phila., Vol. 6, 1852, p. 128.

Cnemidophorus gularis gularis COPE

Ann. Rep. U. S. Nat. Mus., 1898 (1900), p. 602, fig. 118.

TyPe locality: Indianola and San Pedro River, Texas.

RANGE: Arkansas and Oklahoma through Texas west to Arizona, also the northern states of Mexico.

\section{Cnemidophorus hyperythrus hyperythrus (COPE)}

Cnemidophorus hyperythrus CoPE

Proc. Ac. Nat. Sci. Phila., 1863, p. 103.

Verticaria hyperythra hyperythra STEJNEGER and BARBour

Check List N. Amer. Amph. Rept., Ed. 1, 1917, p. 65.

Verticaria hyperythra beldingi STEJNEGER and BARBOUR

Check List N. Amer. Amph. Rept., Ed. 2, 1923, p. 69.

Verticaria espiritensis STEJNEger and Barbour

Check List N. Amer. Amph. Rept., Ed. 2, 1923, p. 68. 
Verticaria hyperythra schmidti STEJNEGER and BARBOUR

Check List N. Amer. Amph. Rept., Ed. 2, p. 1923, p. 69.

Verticaria hyperythra (part) Cope

Ann. Rep. U. S. Nat. Mus., 1898 (1900), p. 563.

TYPE LOCALITY: Cape San Lucas, Lower California.

RANGE: Southern California, Lower California, Cerros, Espiritu

Santo, Magdalena, San Francisco, San Marcos, and Santa

Margarita Islands.

Cnemidophorus hyperythrus caeruleus (DiCKERson)

Verticaria caerulea Dickerson

Bull. Amer. Mus. Nat. Hist., Vol. 41, Oct. 2, 1919, p. 472.

Cnemidophorus hyperythra caeruleus BURT

Bull. U. S. Nat. Mus., No. 154, 1931, p. 240.

TYPE LOCALITY: Carmen Island, Lower California.

RANGE: Same.

Cnemidophorus hyperythrus pictus (Van Denburgh and Slevin)

Verticaria picta VAN Denburgh and Slevvin

Proc. Calif. Ac. Sci., Ser. 4, Vol. 11, July 30, 1921, p. 98.

Cnemidophorus hyperythrus pictus BURT

Bull. U. S. Nat. Mus., No. 154, 1931, p. 242,

TYPE L OCALITY: Monserrate Island, Lower California.

RANGE: Same.

Cnemidophorus hyperythrus danheimae BURT

Cnemidophorus hyperythrus danheimae BurT

Proc. Biol. Soc. Wash., Vol. 42, 1929, p. 154, fig. 33.

Verticaria sericea Van Denburgh

Proc. Calif. Ac. Sci., Ser. 2, Vol. 5, 1895, p. 132, pl. 12.

Verticaria sericea COPE

Ann. Rep. U. S. Nat. Mus., 1898 (1900), p. 564, fig. 103.

TyPE LOCALIty: San José Island, Lower California. RANGE: Same.

Cnemidophorus labialis STEJNEger

Cnemidophorus labialis Stejneger

Proc. U. S. Nat. Mus., Vol. 14, June, 1890, p. 643.

Cnemidophorus labialis CoPE

Ann. Rep. U. S. Nat. Mus., 1898 (1900), p. 610, fig. 122.

TYPE LOCALITY: Cerros Island, Lower California.

RANGE: Cerros Island, Lower California. 


\section{Cnemidophorus maximus Cope}

Cnemidophorus maximus Cope

Proc. Ac. Nat. Sci. Phila., 1863, p. 104.

Cnemidophorus maximus CopE

Ann. Rep. U. S. Nat. Mus., 1898 (1900), p. 570, fig. 104.

TYPE LOCALITY: Cape St. Lucas, Lower California.

RANGE: Southern Lower California.

\section{Cnemidophorus melanostethus CoPE}

Cnemidophorus melanostethus CopE

Proc. Ac. Nat. Sci. Phila., 1863, p. 104.

Cnemidophorus tessellatus melanostethus Cope

Ann. Rep. U. S. Nat. Mus., 1898 (1900), p. 581, fig. 109.

TYPE LOCALity: "Region of the Colorado of California," Colorado Desert, Arizona.

RANGE: Deserts of southern Arizona.

\section{Cnemidophorus perplexus BAIRD and Girard}

Cnemidophorus perplexus BaIRD and GIRARD

Proc. Ac. Nat. Sci. Phila., Vol. 6, 1852, p. 128.

Cnemidophorus tessellatus perplexus (part) CoPE

Ann. Rep. U. S. Nat. Mus., 1898 (1900), p. 573, fig. 106.

tyPe locality: Valley of the Rio San Pedro, tributary of the

Rio Grande del Norte, Texas.

RANGE: Texas and New Mexico.

\section{Cnemidophorus sexlineatus (LINNÉ)}

Lacerta sexlineata LiNNÉ

Syst. Nat., Ed. 12, Vol. 1, 1766, p. 364.

Cnemidophorus sexlineatus DumérIL and BibroN

Erp. Gén., Vol. 5, 1839, p. 1.

Cnemidophorus sexlineatus Cope

Ann. Rep. U. S. Nat. Mus., 1898 (1900), p. 593, fig. 116.

TYPE LOCALITY: In Carolina.

RANGE: Maryland to Florida, west to northern Mexico and Arizona and up the Mississippi Valley as far north as Lake Michigan.

\section{Cnemidophorus tessellatus tessellatus ( $\mathrm{S}_{\mathrm{AY}}$ )}

Ameiva tesselata $\mathrm{SAY}$

Long's Exp. Rocky Mts., Vol. 2, 1823, p. 50.

Cnemidophorus tessellatus tessellatus (part) CopE

Ann. Rep. U. S. Nat. Mus., 1898 (1900), p. 575, fig. 107. 
Cnemidophorus tessellatus BAIRD

Pacif. R. R. Surv., Vol. 10, Pt. 4, 1859, p. 18.

Cnemidophorus dicliersonae STEJnEger and BARBour

Cheek List N. Amer. Amph. Rept., Erl. 2, 1923, p. 70.

Cnemidophorus disparilis STEJNEGER and Barbour

Check List N. Amer. Amph. Rept., Ed. 2, 1923, p. 71.

Cnemidophorus multiscutatus STEJneger ANd Barbour

Check List N. Amer. Ampl. Rept., Ed. 2, 1923, p. 72.

Cnemidophorus punctilinealis Stejneger and BARbour

Check List N. Amer. Amph. Rept., Ed. 2, 1923, p. 72.

Cnemidophorus tessellatus mundus Stejneger and Barbour

Check List N. Amer. Amph. Rept., Ed. 2, 1923, p. 73.

TyPE LOCALITy: Arkansas River, near Castle Rock Creek, Colorado.

RANGE: Texas to California, Utah, Colorado, Nevada, Isla Partida, near Espiritu Santo Island, Lower California, Cerros Island, Tiburon Island, and adjacent region of Sonora (Guaymas).

\section{Cnemidophorus tessellatus canus (VAN Denburgh and Slevin)}

Cnemidophorus canus VAN Denburgh and Slevin

Proc. Calif. Ac. Sci., Ser. 4, Vol. 11, July 30, 1921, p. 97.

Cnemidophorus tessellatus canus BURT

Bull. U. S. Nat. Mus., No. 154, 1931, p. 208.

ty PE LOCAlity: Sal Si Puedes Island, Lower California.

RA N GE: Same, and North and South San Lorenzo Island.

\section{Cnemidophorus tessellatus celeripes (DrCKerson)}

Cnemidophorus celeripes Dickerson

Bull. Amer. Mus. Nat. Hist., Vol. 41, Oct. 2, 1919, p. 472.

Cnemidophorus tessellatus celeripes BurT

Bull. U. S. Nat. Mus., No. 154, 1931, p. 202.

TYPE LOCALITY: San José Island, Lower California. RANGE: Same.

\section{Cnemidophorus tessellatus martyris (STEJNEGER)}

Cnemidophorus martyris STEJNEGER

Proc. U. S. Nat. Mus., Vol. 14, Aug. 31, 1891, p. 407.

Cnemidophorus tessellatus martyris BURT

Bull. U. S. Nat. Mus., No. 154, p. 205.

TyPE LOCALity: San Pedro Martir Island, Gulf of California. RANGE: Type locality. 


\title{
Cnemidophorus tessellatus rubidus COPE
}

Cnemidophorus tessellatus rubidus COPE

Trans. Amer. Philos. Soc., Ser. \&, Vol. 17, 1892, p. 36, pl. 12, fig. 1.

Cnemidophorus vandenburghi STEJNEGER and BARBOUR

Check List N. Amer. Amph. Rept., Ed. 2, 1923, p. 74.

Cnemidophorus tessellatus rubidus COPE

Ann. Rep. U. S. Nat. Mus., 1898 (1900), p. 584, fig. 110.

TyPE LOCALITy: Sta. Margarita Island, Lower California.

RANGE: Carmen, Sta. Margarita and Magdalena Islands, Lower

California.

\section{Cnemidophorus tessellatus stejnegeri (Van Denburgh)}

Cnemidophorus stejnegeri $\mathrm{V}_{\text {AN }}$ DenBurgh

Proc. Calif. Ac. Sci., Ser. 2, Vol. 4, 1894, p. 300.

Cnemidophorus tessellatus stejnegeri STEJNEGER and BARBour

Check List N. Amer. Amph. Rept., Ed. 1, 1917, p. 68.

Cnemidophorus grahamii stejnegerii Cope

Ann. Rep. U. S. Nat. Mus., 1898 (1900), p. 599.

TyPE LOCAlity: Near Ensenada, Lower California.

RANGE: Southern California and northern Lower California.

\author{
SCINCIDAE $(F A M I L Y)$ \\ Leiolopisma ( $G E N U S$ ) \\ Duméril and Bibron, Erp. Gén., Vol. 5, 1839, p. 742 \\ TYPE: telfairii
}

\section{Leiolopisma laterale ( $\left.\mathrm{S}_{A Y}\right)$}

Scincus lateralis $\mathrm{S}_{\mathrm{AY}}$

Long's Exp. Rocky Mts., Vol. 2, 1923, p. 324.

Leiolopisma laterale JoRDAN

Man. Vertebr. North. U. S., Ed. 8, 1899, p. 201.

Liolepisma laterale COPE

Ann. Rep. U. S. Nat. Mus., 1898 (1900), p. 622, fig. 123.

TYPE LOCALITY: Banks of Mississippi River below Cape Girardeau, Missouri.

RANGE: New Jersey to Florida, west to Illinois, Kansas, Oklahoma, and Texas. 


\section{Eumeces $(G E N U S)^{1}$}

Wiegmann, Herp. Mexicana, 1834, p. 36

TYPE: pavimentatus

\section{Eumeces anthracinus (BAIRD)}

Plestiodon anthracinus $\mathrm{BAIRD}$

Journ. Ac. Nat. Sci. Phila., Ser. 2, Vol. 1, 1849, fig. 294.

Eumeces anthracinus CoPE

Bull. U. S. Nat. Mus., No. 1, 1875, p. 45.

Eumeces anthracinus COPE

Ann. Rep. U. S. Nat. Mus., 1898 (1900), p. 661, fig. 135.

TyPe Locality: North Mountain near Carlisle, Pennsylvania.

RANGE: New York to North Carolina and Missouri, Arkansas, Kansas, and Texas.

\section{Eumeces brevilineatus Cope}

Eumeces brevilineatus COPE

Bull. U. S. Nat. Mus., No. 17, 1880, p. 18.

Eumeces brevilineatus CoPE

Ann. Rep. U. S. Nat. Mus., 1898 (1900), p. 664, fig. 137.

Ty PE Locality: Helotes, Bexar Co., Texas.

RANGE: Texas.

\section{Eumeces callicephalus BocourT}

Eumeces callicephalus BocourT

Miss. Sci. Mex., Rept., 1879, p. 431, pl. 22 D, fig. 2, pl. 22 E, fig. 2.

Eumeces callicephalus 'TAYLOR

Univ. Kansas Sci. Bull., Vol. 19, No. 7, Nov. 1929, p. 67.

TYPE LOCALity: Guanajuato, Mexico.

RANGE: Table land of North Central Mexico north to the Huachuca Mountains, Arizona.

\section{Eumeces egregius (BAIRD)}

Plestiodon egregius BAIRD

Proc. Ac. Nat. Sci. Phila., 1858, p. 256.

Eumeces egregius COPE

Bull. U. S. Nat. Mus., No. 1, 1875, p. 45.

Eumeces egregius CoPE

Ann. Rep. U. S. Nat. Mus., 1898 (1900), p. 655, fig. 132.

1 Two additional species of Eumeces, viz. E. inexpectatus and E. laticeps, have been recently recognized by E. H. Taylor (Univ. Kansas Science Bull., Vol. 22, No. 13, 1932, p. 251, and Univ. Kansas Science Bull., Vol. 29, No. 13, 1932, p. 263). The evidence thus far adduced does not support the validity of these forms. 
TYPE LOCALITY: Indian Key, Florida.

RANGE: Western Florida and the Florida Keys north to Alachua County.

\section{Eumeces fasciatus (LINNÉ)}

BLUE-TAILED SKINK, SCORPION

Lacerta fasciata LINNÉ

Syst. Nat., Ed. 10, Vol. 1, 1758, p. 209.

Eumeces fasciatus COPE

Bull. U. S. Nat. Mus., No. 1, 1875, p. 45.

Eumeces quinquelineatus COPE

Ann. Rep. U. S. Nat. Mus., 1898 (1900), p. 632, fig. 125.

TYPE LOCALITY: Carolina.

RANGE: Southern New England to Florida, up the Mississippi

Valley to Canada and westward to Arizona.

\section{Eumeces humilis BOULENGER}

Eumeces humilis Boulenger

Cat. Liz. Brit. Mus., Vol. 3, 1887, p. 377.

Eumeces humilis Mosaver

Occ. Pap. Mus. Zool., Univ. Michigan, No. 246, June 9, 1932, p. 10, pl. 1, fig. 3.

Ty Pe locality: Presidio (near Mazatlan), Mexico.

RANGE: Known from west coast of Mexico; has recently been found in the Guadalupe Mountains, Texas.

\section{Eumeces longirostris (COPE)}

Plestiodon longirostris Cope

Proc. Ac. Nat. Sci. Phila., 1861, p. 313.

Eumeces longirostris $\mathrm{COPE}$

Bull. U. S. Nat. Mus., No. 1, 1875, p. 45.

Eumeces longirostris Cope

Ann. Rep. U. S. Nat. Mus., 1898 (1900), p. 631, fig. 124.

TYPE LOCALITY: Bermuda.

RANGE: Bermuda.

\section{Eumeces multivirgatus (HaLlowell)}

Plestiodon multivirgatum Hallowell

Proc. Ac. Nat. Sci. Phila., 1857, p. 215.

Eumeces multivirgatus $\mathrm{COPE}$

Bull. U. S. Nat. Mus., No. 1, 1875, p. 45.

Eumeces epipleurotus COPE

Ann. Rep. U. S. Nat. Mus., 1898 (1900), p. 650, fig. 129.

Eumeces leptogrammus Cope

Ann. Rep. U. S. Nat. Mus., 1898 (1900), p. 651, fig. 130. 
Eumeces multivirgatus CoPE

Ann. Rep. U. S. Nat. Mus., 1898 (1900), p. 653, fig. 131.

Ty PE Locality: Posa Creek, 460 miles west of Fort Riley, Kansas.

RAnge: Nebraska to northern Texas; Colorado and New Mexico.

Eumeces obsoletus (BAIRD and Girard)

Plestiodon obsoletum $\mathrm{B}_{\mathrm{AIRD}}$ and Girard

Proc. Ac. Nat. Sci. Phila., Vol. 6, 1852, p. 129.

Eumeces obsoletus Cope

Bull. U. S. Nat. Mus., No. 1, 1875, p. 4.5.

Eumeces guttulatus STEJNEger and Barbour

Check List N. Amer. Amph. Rept., Ed. 2, 1923, p. 75.

Eumeces obsoletus Cope

Ann. Rep. U. S. Nat. Mus., 1898 (1900), p. 646, fig. 128.

TYPE LOCALITy: Valley of the Rio San Pedro, tributary of the

Rio Grande del Norte, Texas.

RANGE: Utah and Kansas south to Northern Mexico.

\section{Eumeces pachyurus Cope}

Eumeces pachyurus Cope

Bull. U. S. Nat. Mus., No. 17, 1880, p. 19.

Eumeces pachyurus CoPE

Ann. Rep. U. S. Nat. Mus., 1898 (1900), p. 659.

TyPE Locality: Near Dallas, Texas.

RANGE: Oklahoma and Texas.

\section{Eumeces pluvialis Cope}

Eumeces pluvialis CoPE

Bull. U. S. Nat. Mus., No. 17, 1880, p. 19.

Eumeces pluvialis Cope

Ann. Rep. U. S. Nat. Mus., 1898 (1900), p. 663, fig. 136.

TYPE LoCAlity: Mobile, Alabama.

RANGE: Central Gulf area.

\section{Eumeces septentrionalis (BAIRD)}

Plestiodon septentrionalis BAIRD

Proc. Ac. Nat. Sci. Phila., 1858, p. 256.

Eumeces septentrionalis CopE

Bull. U. S. Nat. Mus., No. 1, 1875, p. 44.

Eumeces septentrionalis CoPE

Ann. Rep. U. S. Nat. Mus., 1898 (1900), p. 656, fig. 133.

TYPE LOCALITY: Minnesota and Nebraska.

RANGE: Minnesota, Iowa, Nebraska, Kansas and Oklahoma. 


\section{Eumeces skiltonianus (BAIRD and GIRARD)}

Plestiodon skiltonianum BAIRD and GIRARD

Stansbury's Expl. Surv. Vall. Great Salt Lake, 1852, p. 349, pl. 4, figs. 4-6.

Eumeces skiltonianus COPE

Bull. U. S. Nat. Mus., No. 1, 1875, p. 45.

Eumeces lagunensis STEJNEgER and Barbour

Check List N. Amer. Amph. Rept., Ed. 2, 1923, p. 75.

Eumeces shiltonianus Cope

Ann. Rep. U. S. Nat. Mus., 1898 (1900), p. 640, fig. 126.

TYPE LOCALITY: Oregon.

RANGE: Washington, western Oregon, and most of California, except the extreme southwestern desert area; Lower California; eastward through Nevada to Utah.

\section{Eumeces tetragrammus (BAIRD)}

Plestiodon tetragrammus $\mathrm{B}_{\mathrm{AIRD}}$

Proc. Ac. Nat. Sci. Phila., 1858, p. 256.

Eumeces tetragrammus Cope

Bull. U. S. Nat. Mus., No. 1, 1875, p. 45.

Eumeces tetragrammus Cope

Ann. Rep. U. S. Nat. Mus., 1898 (1900), p. 660.

TYPE LOCALITY: Lower Rio Grande.

RANGE: Southern Texas and northeastern Mexico.

Neoseps ( $G E N U S)$

Stejneger, Proc. U. S. Nat. Mus., Vol. 39, Sept. 23, 1910, p. 33 TYPE: reynoldsi

\section{Neoseps reynoldsi STEJNEGER}

Neoseps reynoldsi STEJNEgER

Proc. U. S. Nat. Mus., Vol. 39, Sept. 23, 1910, p. 34.

тy pe Locality: Near Spring Lake, Fruitland Park, Lake Co.,

Florida.

RANGE: Central Florida.

AMPHISBAENIDAE (FAMILY)

\section{Bipes ( $G E N U S$ )}

Latreille, Hist. Nat. Rept., Vol. 2, 1802, p. 90

TXPE: canaliculatus

\section{Bipes biporus (COPE)}

Euchirotes biporus Cope

Amer. Nat., Vol. 28, 1894, p. 436, figs. 5a-e. 
Bipes biporus STEJNEGER and BARBour

Check List N. Amer. Amph. Rept., Ed. 1, 1917, p. 72.

Euchirotes biporus Cope

Ann. Rep. U. S. Nat. Mus., 1898 (1900), p. 680, fig. 140.

TYPE LoCality: La Paz, Lower California.

RANGE: Southern Lower California.

\section{Rhineura ( $G E N U S$ )}

Cope, Proc. Ac. Nat. Sci. Phila., 1861, p. 75

TYPE: floridana

Rhineura floridana (BAIRD)

Lepidosternon floridanum $\mathrm{B}_{\mathrm{AIRD}}$

Proc. Ac. Nat. Sci. Phila., 1858, p. 255.

Rhineura floridana Cope

Proc. Ac. Nat. Sei. Phila., 1861, p. 75.

Rhineura floridana Cope

Ann. Rep. U. S. Nat. Mus., 1898 (1900), p. 686, fig. 141.

TYPE LOCALITY: Micanopy, Florida.

RANGE: Northern and central Florida.

\section{SERPENTES ( $S U B O R D E R)$}

Linné, Syst. Nat., Ed. 10, Vol. 1, 1758, p. 214

\section{LEPTOTYPHLOPIDAE (FAMILY)}

\section{Leptotyphlops ( $G E N U S$ )}

Fitzinger, Syst. Rept., 1843, p. 24

TYPE: nigricans

\section{Leptotyphlops dulcis (BAIRD and Girard)}

Rena dulcis BAIRD and GLRARD

Cat. N. Amer. Rept., Pt. 1, 1853, p. 142.

Leptotyphlops dulcis STEJNEGER

Proc. U. S. Nat. Mus., Vol. 14, Oct. 31, 1891, p. 501.

Glauconia dulcis Cope

Ann. Rep. U. S. Nat. Mus., 1898 (1900), p. 717, fig. 143.

TyPe Locality: Between San Pedro and Comanche Springs,

Texas.

RANGE: Northern Mexico, Texas, Oklahoma and New Mexico. 
Leptotyphlops humilis humilis (BAIRD and GIRARD)

Rena humilis BAIRD and GIRARD

Cat. N. Amer. Rept., Pt. 1, 1853, p. 143.

Leptotyphlops humilis humilis KLAUBER

Trans. San Diego Nat. Hist. Soc., Vol. 6, No. 23, July 8, 1931, p. 343.

Glauconia humilis CopE

Ann. Rep. U. S. Nat. Mus., 1898 (1900), p. 719, fig. 144.

Siagonodon humilis STEJNEGER and Barbour

Check List N. Amer. Amph. Rept., Ed. 2, 1923, p. 79.

TYPE LOCALITY: Valliecitas [sic], California.

RANGE: San Diego Co., California from the coast to the crest of the divide.

\section{Leptotyphlops humilis cahuilae KLAUBER}

Leptotyphlops humilis cahuilae KLAUBER

Trans. San Diego Nat. Hist. Soc., Vol. 6, No. 23, July 8, 1931, p. 339.

TyPe Locality: Yaqui Well, San Diego Co., California.

RANGE: Colorado and Yuma Deserts of California and Arizona.

Leptotyphlops humilis slevini KLAUBER

Leptotyphlops humilis slevini KLAUBER

Trans. San Diego Nat. Hist. Soc., Vol. 6, No. 23, July 8, 1931, p. 338.

ty Pe locality: La Paz, Lower California.

RANGE: Cape Region of Lower California.

\section{Leptotyphlops myopica (GARMAN)}

Stenostoma myopicum GARMaN

Mem. Mus. Comp. Zoöl., Vol. 8, No. 3, 1883, p. 6.

Glauconia dissecta COPE

Ann. Rep. U. S. Nat. Mus., 1898 (1900), p. 716, fig. 142.

TYPE LOCAlity: Near Tampico, Mexico.

RANGE: Northern Mexico into southern New Mexico.

BOIDAE ( FAMILY)

Lichanura ( $G E N U S)$

Cope, Proc. Ac. Nat. Sci. Phila., 1861, p. 304

TYPE: trivirgata

Lichanura roseofusca roseofusca (COPE)

Lichanura roseofusca CoPE

Proc. Ac. Nat. Sci. Phila., 1868, p. 2. 
Lichanura roseofusca roseofusca KLLAUBER

'Trans. San Diego Soc. Nat. Hist., Vol. 6, No. 20, Apr. 30, 1931, p. 316.

Lichanura roseofusca Cope

Ann. Rep. U. S. Nat. Mus., 1898 (1900), p. 724, fig. 146.

TYPE LOCALITY: Northern Lower California.

RANGE: Southwestern California and northern Lower California.

Lichanura roseofusca gracia KLAUBER

Lichanura roseofusca gracia KLAUBER

Trans. San Diego Soc. Nat. Hist., Vol. 6, No. 20, Apr. 30, 1931, p. 307.

ty pe locality: Randsburg, Kern Co., California.

RANGE: Desert regions of southeastern California and southwestern Arizona.

\section{Lichanura trivirgata CoPE}

Lichanura trivirgata $\mathrm{COPE}$

Proc. Ac. Nat. Sci. Phila., 1861, p. 304.

Lichanura trivirgata COPE

Ann. Rep. U. S. Nat. Mus., 1898 (1900), p. 723, fig. 145.

TyPe Locality: Cape St. Lucas, Lower California.

RANGE: Southern Lower California.

\section{Charina ( $G E N U S)$}

Gray, Cat. Snakes Brit. Mus., 1849, p. 113

TYPE: bottae

Charina bottae (BLAINville)

RUBBER-SNAKE, BALL-SNAKE

Tortrix bottae BLAINVILLE

Nouv. Ann. Mus. Hist. Nat. Paris, Vol. 4, 1835, p. 289, pl. 26, figs. 1-1B.

Charina bottae Gray

Cat. Snakes Brit. Mus., 1849, p. 113.

Charina bottae Cope

Ann. Rep. U. S. Nat. Mus., 1898 (1900), p. 728, fig. 148.

TYPE LOCALITY: California.

RANGE: Humid districts of California south to San Bernardino

Mountains, Nevada, Idaho, Oregon, and Washington, east to

Montana, Wyoming, and Utah. 


\section{COLUBRIDAE (FAMILY)}

Carphophis ( $G E N U S)$

Gervais, D'Orbigny's Dict. Univ. Hist. Nat., Vol. 3, 1843, p. 191

TYPE: ainoena

WORM - SN A KES

\section{Carphophis amoena amoena ( $\left.\mathrm{SAY}_{\mathrm{A}}\right)$}

Coluber amoenus $\mathrm{S}_{\mathrm{AY}}$

Journ. Ac. Nat. Sci. Phila., Vol. 4, Pt. 2, 1825, p. 237.

Carphophis amoena amoena BLANCHARD

Papers Mich. Acad. Sci. Arts Letters, Vol. 4, 1924, p. 527.

Carphophiops amoenus CopE

Ann. Rep. U. S. Nat. Mus., 1898 (1900), p. 735, fig. 151.

Ty

RANGE: Connecticut to New York, south to Florida, and west into the Appalachian Mountains.

\section{Carphophis amoena helenae (KeNNICOTT)}

Celuta helenae KenNicotT

Proc. Acad. Nat. Sci. Phila., 1859, p. 100.

Carphophis amoena helenae $\mathrm{BLANCHARD}$

Papers Mich. Acad. Sci. Arts Letters, Vol. 4, 1924, p. 527.

TyPE LOCALIty: Monticello, Mississippi.

RANGE: Central Illinois south to Mississippi, east to northwest Alabama, the Tennessee Valley to eastern Tennessee, West Virginia and eastern Ohio.

\section{Carphophis amoena vermis (KENNICOTT)}

Celuta vermis KenNicotT

Proc. Acad. Nat. Sci. Phila., 1859, p. 99.

Carphophis amoena vermis BLANCHARD

Papers Mich. Acad. Sci. Arts Letters, Vol. 4, 1924, p. 527.

Carphophiops vermis CoPE

Ann. Rep. U. S. Nat. Mus., 1898 (1900), p. 737, fig. 152.

TyPE LOCALity: Missouri.

RANGE: Southeastern Nebraska and central Missouri through eastern Oklahoma, Arkansas, Louisiana, Kansas and Texas. 


\section{Abastor ( $G E N U S)$ \\ Gray, Cat. Snakes Brit. Mus., 1849, p. 78 \\ TYPE: erythrogrammus}

Abastor erythrogrammus (DAUDIN)

RAINBOW-SNAKE:

Coluber erythrogrammus DAUDIN

Hist. Nat. Rept., Vol. 7, 1803, p. 93, pl. 83, figs. 2a-b.

Abastor erythrogrammus GRAY

Cat. Snakes Brit. Mus., 1849, p. 78.

Abastor erythrogrammus COPE

Ann. Rep. U. S. Nat. Mus., 1898 (1900), p. 738, fig. 153.

TYPE LOCALITY: États Unis d'Amérique.

RANGE: Virginia southward to Florida and Alabama.

\section{Farancia ( $G E N U S)$}

Gray, Zool. Misc., 1832, p. 78

TYPE: drummond $i=$ abacura

Farancia abacura (HoLBRook)

HORN-SNAKE, MUD-SNAKE

Coluber abacurus HoLBRooK

N. Amer. Herp., Ed. 1, Vol. 1, 1836, p. 119, pl. 23.

Farancia abacura BAIRD and GIRARD

Cat. N. Amer. Rept., Pt. 1, 1853, p. 123.

Farancia abacura COPE

Ann. Rep. U. S. Nat. Mus., 1898 (1900), p. 741, fig. 154.

TYPE LOCALITY: South Carolina.

RANGE: Virginia to Florida and westward to Louisiana and Texas,

in the Mississippi Valley northward to Indiana.

\section{Diadophis ( $G E N U S$ )}

Baird and Girard, Cat. N. Amer. Rept., Pt. 1, 1853, p. 112 TYPE: punctatus

\section{RING-NECK SNAKES}

Diadophis amabilis amabilis (BAIRD and GIRARD)

Diadophis amabilis BAIRD and GIRARD

Cat. N. Amer. Rept., Pt. 1, 1853, p. 113.

Diadophis amabilis amabilis BLANCHARD

Occ. Papers, Mus. Zool. Univ. Michigan, No. 142, July 9, 1923, p. 3. Diadophis amabilis COPE

Ann. Rep. U. S. Nat. Mus., 1898 (1900), p. 746, figs. 157, 160. 
TyPE LOCALity: San José, California.

RANGE: California, restricted to the regions about San Francisco and the San Joachin and Sacramento Valleys.

Diadophis amabilis modestus (DUMÉRIL and BocourT)

Diadophis punctatus var. modestus DumÉril and BocourT

Miss. Sci. Mexique, Vol. 3, Pt. 10, 1886, p. 623.

Diadophis amabilis modestus BLANCHARD

Occ. Papers Mus. Zool. Univ. Michigan, No. 142, July 9, 1923, p. 8.

TYPE LOCALITY: California.

RANGE: San Bernardino Mountains, Los Angeles County, and

Santa Catalina Island, California.

Diadophis amabilis occidentalis BLANCHARD

Diadophis amabilis occidentalis BLANCHARD

Occ. Papers Mus. Zool. Univ. Michigan, No. 142, July 9, 1923, p. 6.

TYPE LOCALITY: Bridgeville, Humboldt County, California.

RANGE: From Sonoma County, northward perhaps to the Columbia River.

Diadophis amabilis pulchellus (BAIRD and GIRARD)

Diadophis pulchellus BAIRD and GIRARD

Cat. N. Amer. Rept., Pt. 1, 1853, p. 115.

Diadophis amabilis pulchellus BlanchaRD

Oce. Papers Mus. Zool. Univ. Michigan, No. 142, July 9, 1923, p. 8. TYPE LOCALITY: El Dorado County, California.

RANGE: Western slopes of the Sierra Nevada, south perhaps to

Tejon Pass in California and north perhaps to southern Oregon.

Diadophis amabilis similis BLANCHARD

Diadophis amabilis similis BLANCHARD

Occ. Papers Mus. Zoöl. Univ. Michigan, No. 142, July 9, 1923, p. 4. TYPE LOCALITY: San Diego, California.

RANGE: From southwestern San Bernadino County south into the San Pedro Martir range in Lower California.

\section{Diadophis amabilis vandenburghi BLANCHARD}

Diadophis amabilis vandenburghi BLANCHARD

Occ. Papers Mus. Zool. Univ. Michigan, No. 142, July 9, 1923, p. 5. TYPE LOCALITY: Carmel, Monterey County, California. RANGE: Ventura to Santa Cruz County, California. 
Diadophis anthonyi Van Denburgi and Slevin

Diadophis anthonyi $\mathrm{V}_{\mathrm{AN}}$ Dendurgir and SLevin

Proc. Calif. Ac. Sci., Ser. 4, Vol. 13, No. 1, July 25, 1923, p. 1.

TYPE LOCALITY: South Todos Santos Island, Lower California.

RANGE: Same.

Diadophis punctatus punctatus (LINNÉ)

Coluber punctatus LinNé

Syst. Nat., Ed. 12, Vol. 1, 1766, p. 376.

Diadophis punctatus BAIRD and GIRARD

Cat. N. Amer. Rept., Pt. 1, 1853, p. 112.

Diadophis punctatus punctatus BARBOUR

Proc. N. Engl. Zoöl. Club, Vol. 7, 1919, p. 7.

Diadophis punctatus COPE

Ann. Rep. U. S. Nat. Mus., 1898 (1900), p. 751, fig. 162.

TYPE LOCALITY: Carolina.

RANGE: Southeastern states lowlands from Florida, northward to northern North Carolina from sea-level to about 2500 feet, possibly higher in the mountains of South Carolina and Georgia.

\section{Diadophis punctatus arnyi (KenNicotT)}

Diadophis arnyi KENNICOTT

Proc. Ac. Nat. Sci. Phila., 1859, p. 99.

Diadophis regalis arnyi $\mathrm{COPE}$

Ann. Rep. U. S. Nat. Mus., 1898 (1900), p. 745, fig. 156.

TYPE LOCALity: Hyatt, Anderson County, Kansas.

RANGE: Ohio, northern Illinois, Indiana, Kansas, Iowa, Missouri, northwestern Arkansas, Oklahoma, and Texas.

\section{Diadophis punctatus edwardsii (MERREM)}

Coluber edwardsii Merrem

Tent. Syst. Amphib., 1820, p. 136.

Diadophis punctatus edwardsii BARBOUR

Proc. N. Eng. Zoöl. Club, Vol. 7, 1919, p. 9.

TYPE LOCALITY: Pennsylvania.

RA NGE: The mountains of the Carolinas through the Appalachians northward to the Northern Peninsula of Michigan, Wisconsin, southern Canada, and the Maritime Provinces.

\section{Diadophis punctatus stictogenys CoPE}

Diadophis punctatus var. stictogenys CopE

Proc. Ac. Nat. Sci. Phila., 1860, p. 250. 
TYPE LOCALITY: Unknown.

RANGE: Southern Illinois, through the lower Mississippi Valley to the Gulf.

Diadophis regalis regalis (BAIRD and GIRARD)

Diadophis regalis $\mathrm{B}_{\mathrm{AIRD}}$ and GIRARD

Cat. N. Amer. Rept., Pt. 1, 1853, p. 115.

Diadophis regalis regalis BLANCHARD

Occ. Papers Mus. Zool. Univ. Michigan, No. 142, July 9, 1923, p. 1. Diadophis regalis Cope

Ann. Rep. U. S. Nat. Mus., 1898 (1900), p. 744, fig. 155.

TYPE LOCALity: Sonora, Mexico.

RANGE: Southern Utah, southeastern Arizona, to central Texas and to the state of Vera Cruz, Mexico.

\title{
Diadophis regalis arizonae BLANCHARD
}

Diadophis regalis arizonae BLANCHARD

Occ. Papers Mus. Zool. Univ. Michigan, No. 142, July 9, 1923, p. 2. TyPe locality: Sabino Cañon, Arizona.

RANGE: Central Arizona to northern Sonora.

\author{
Heterodon ( $G E N U S)$ \\ Latreille, Hist. Nat. Rept., Vol. 4, 1802, p. 32 \\ TYPE: platirhinos $=$ contortrix \\ HOG-NOSE SNAKES
}

Heterodon browni STEJNEGER

Heterodon browni STEJNEGER

Proc. Biol. Soc. Washington, Vol. 16, 1903, p. 123.

TYPE LOCALITY: Lemon City, Florida.

RANGE: Extreme southern Florida.

Heterodon contortrix (LINNÉ)

PUFFING ADDER, SPREADING ADDER

Boa contortrix LinNé

Syst. Nat., Ed. 12, Vol. 1, 1766, p. 373.

Heterodon contortrix STEJNEGER and BARBOUR

Check List N. Amer. Amph. Rept., Ed. 1, 1917, p. 76.

Heterodon platyrhinus COPE

Ann. Rep. U. S. Nat. Mus., 1898 (1900), p. 761, fig. 165.

TYPE LOCALITY: Carolina.

RANGE: Massachusetts to Florida westward to Texas and northward through the states of the Mississippi basin to Minnesota. 
Heterodon nasicus BAIRD and GIRARD

Heterodon nasicus $\mathrm{B}_{\mathrm{AIRD}}$ and GIRARD

Stansbury's Expl. Surv. Vall. Great Salt Lake, 1852, p. 352.

Heterodon nasicus COPE

Ann. Rep. U. S. Nat. Mus., 1898 (1900), p. 772, fig. 168.

TYPE LOCALITY: Texas.

RANGE: Southwestern Iowa, Kansas and Nebraska west to Montana and south to northern Mexico. Common along the Mexican border from Texas to Arizona.

Heterodon simus (LINNÉ)

Coluber simus LiNNÉ

Syst. Nat., Ed. 12, Vol. 1, 1766, p. 375.

IIeterodon simus HoLBrook

N. Amer. Herp., Ed. 2, Vol. 4, 1842, p. 57, pl. 15.

Heterodon simus COPE

Ann. Rep. U. S. Nat. MIus., 1898 (1900), p. 770, fig. 167.

TYPE LOCALITY: Carolina.

RANGE: Southeastern States, Indiana to Florida.

Liopeltis ( $G E N U S)$

Fitzinger, Syst. Rept., 1843, p. 26

TYPE: tricolor

Liopeltis vernalis (HARLAN)

SMOOTH GREEN-SNAKE

Coluber vernalis HaRLAN

Journ. Ac. Nat. Sci. Phila., Vol. 5, 1827, p. 361.

Liopeltis vernalis COPE

Proc. Ac. Nat. Sci. Phila., 1860, p. 560.

Liopeltis vernalis $\mathrm{COPE}$

Ann. Rep. U. S. Nat. Mus., 1898 (1900), p. 782, fig. 169.

type LOCality: Pennsylvania and New Jersey are mentioned as habitat.

RANGe: Southern Canada through the Eastern and Middle States south to Florida and Texas, New Mexico. North Dakota and Colorado. 
Opheodrys ( $G E N U S$ )

Fitzinger, Syst. Rept., 1843, p. 26

TYPE: aestivus

Opheodrys aestivus (LINNÉ)

ROUGH GREEN-SNAKE

Coluber aestivus LiNNÉ

Syst. Nat., Ed. 12, Vol. 1, 1766, p. 387.

Opheodrys aestivus COPE

Proc. Ac. Nat. Sci. Phila., 1860, p. 560.

Cyclophis aestivus CopE

Ann. Rep. U. S. Nat. Mus., 1898 (1900), p. 784, fig. 170.

TYPE LOCALITY: Carolina.

RANGE: Connecticut to Florida, westward to New Mexico, in the

Mississippi drainage basin found in the southern portion and northward to Kansas and Illinois.

\section{Coluber ( $G E N U S)$}

Linné, Syst. Nat., Ed. 10, Vol. 1, 1758, p. 216

TYPE: constrictor

\section{Coluber anthonyi (STEJNEGER)}

Bascanion anthonyi STEJNEGER

Proc. U. S. Nat. Mus., Vol. 23, June 5, 1901, p. 715.

Coluber anthonyi Stejneger and BARBour

Check List N. Amer. Amph. Rept., Ed. 1, 1917, p. 78.

TYPE LOCALITY: Clarion Island, Revilla Gigedo group, off west coast of Mexico.

RANGE: Clarion Island, Revilla Gigedo group, off west coast of Mexico.

\section{Coluber aurigulus (Cope)}

Drymobius aurigulus COPE Proc. Ac. Nat. Sci. Phila., 1861, p. 301.

Coluber aurigulus StEJNEgER and BARBour

Check List N. Amer. Amph. Rept., Ed. 1, 1917, p. 78.

Zamenis aurigulus COPE

Ann. Rep. U. S. Nat. Mus., 1898 (1900), p. 810, fig. 179.

Ty PE Locality: Cape St. Lucas, Lower California.

RANGE: Southern Lower California. 
Coluber barbouri Van Denburgir and Slevin

Coluber barbouri Van Denburgir and Slevin

Proc. Calif. Ac. Sci., Ser. 4, Vol. 11, July 30, 1921, p. 98.

Type locality: Isla Partida, Espiritu Santo Island, Lower California.

RANGE: Same.

\section{Coluber constrictor constrictor (LiNNÉ)}

BLACK-SNAKE

Coluber constrictor (part) LINNÉ

Syst. Nat., Ed. 10, Vol. 1, 1758, p. 216.

Zamenis constrictor (part) COPE

Ann. Rep. U. S. Nat. Mus., 1898 (1900), p. 791, fig. 171.

TYPE LOCALITY: "Habitat in America septentrionale."

RANGE: The whole of the eastern United States, westward to

Texas and the Great Plains.

Coluber constrictor flaviventris ( $\mathrm{S}_{\mathrm{AY}}$ )

WESTERN OR BLUE RACER

Coluber flaviventris $\mathrm{S}_{\mathrm{AY}}$

Long's Exp. Rocky Mts., Vol. 1, 1823, p. 185.

Coluber constrictor flaviventris GaRMaN

Mem. Mus. Comp. Zoöl., Vol. 8, No. 3, 1883, p. 147.

TYPE LOCALITY: Stone quarry on west side of Missouri River

three miles above the mouth of Boyer's River.

RANGE: Central and southwestern States.

Coluber constrictor mormon (BAIRD and GIRARD)

Coluber mormon BAIRD and Girard

Stansbury's Expl. Surv. Vall. Great Salt Lake, Append. C, Rept., 1852, p. 351.

Coluber constrictor mormon Van Denburgh and Slevin

Proc. Calif. Ac. Sci., Vol. 11, 1921, p. 28.

TYPE LOCALITY: Valley of the Great Salt Lake, Utah.

RANGE: From the Pacific Ocean east to Montana, south into California, Idaho, Nevada and Utah.

Coluber flagellum flagellum (SHAw)

COACH WHIP

Coluber flagellum SHAw

Gen. Zool., Vol. 3, 1802, p. 475.

Zamenis flagellum flagellum COPE

Ann. Rep. U. S. Nat. MIus., 1898 (1900), p. 799, fig. 174. 
Ty PE LOCALITy: Carolina and Virginia.

RANGE: Virginia to Florida, westward to the Rocky Mountains.

Coluber flagellum flavi-gularis (HALLOWELL)

Psammophis flavi-gularis HALlowell

Proc. Acad. Nat. Sci. Phila., 1852, p. 178.

Masticophis flagellum flavigularis ORTENBURGER

Mem. Univ. Mich. Mus., Vol. 1, 1928, p. 92.

TyPE LOCAlity: Cross Timbers, Indian Territory.

RANGE: Northern Kansas, southeastern Colorado south to central Mexico.

\section{Coluber flagellum frenatum (STEJNEGER)}

Bascanion flagellum frenatum STEJNEGER

N. Am. Fauna, No. 7, 1893, p. 208.

Coluber flagellum frenatum ORTENBURGER

Mem. Univ. Mich. Mus., Vol. 1, 1928, p. 112.

TYPE LoCality: Mountain Springs, Colorado Desert, San Diego Co., California.

RANGE: Central and southern California, Lower California.

Nevada, Utah, Arizona and Sonora to Sinaloa.

\section{Coluber piceus (COPE)}

Bascanium piceum Cope

Proc. U. S. Nat. Mus., Vol. 14, March 28, 1892, p. 625.

Coluber flagellum piceus Stejneger and Barbour

Check List N. Amer. Amph. Rept., Ed. 1, 1917, p. 79.

Zamenis flagellum piceus $\mathrm{CoPE}$

Ann. Rep. U. S. Nat. Mus., 1898 (1900), p. 804, fig. 175.

type locality: Camp Grant, Arizona.

RANGE: Southern Arizona and Lower California.

Coluber lateralis (HALLOWELL)

Leptophis lateralis HaLLOWELL

Proc. Ac. Nat. Sci. Phila., Vol. 6, 1853, p. 237.

Coluber lateralis StejNeger and Barbour

Check List N. Amer. Amph. Rept., Ed. 1, 1917, p. 80.

Zamenis lateralis $\mathrm{COPE}$

Ann. Rep. U. S. Nat. Mus., 1898 (1900), p. 807, fig. 177.

TYPE LOCALITY: California.

RANGE: Southern and Lower California. 


\section{Coluber ruthveni (ORTENBURGER)}

Masticophis ruthveni Ortenburger

Occ. Papers Mus. Zool. Univ. Michigan, No. 139, July 9, 1923, p. 3, pl. 1-3.

TYPE LOCALity: Brownsville, Texas.

RANGE: Southeastern Texas and Tamaulipas, Mexico.

Coluber schotti (BAIRD and GIRARD)

Masticophis schotti BaIRD and GIRARD

Cat. N. Amer. Rept., Pt. 1, 1853, p. 160.

Coluber schotti STEJNEGER and Barbour

Check List N. Amer. Amph. Rept., Ed. 1, 1917, p. 80.

Zamenis schotti COPE

Ann. Rep. U. S. Nat. Mus., 1898 (1900), p. 811, fig. 18a.

Ty Pe locality: Eagle Pass, Texas.

RANGE: Southern Texas and northeastern Mexico.

\section{Coluber semilineatus (COPE)}

Bascanium semilineatum CoPE

Proc. U. S. Nat. Mus., Vol. 14, March 28, 1892, p. 626.

Coluber semilineatus STEJNEGER and BARBOUR

Check List. N. Amer. Amph. Rept., Ed. 1, 1917, p. 80.

Zamenis semilineatus COPE

Ann. Rep. U. S. Nat. Mus., 1898 (1900), p. 805, fig. 176.

ty

RANGE: Arizona and the adjacent territory of Mexico.

Coluber taeniatus taeniatus (HALLOwELL)

Leptophis taeniata HaLlowell

Proc. Ac. Nat. Sci. Phila., Vol. 6, 1852, p. 181.

Coluber taeniatus Garman

Mem. Mus. Comp. Zoöl., Vol. 8, No. 3, 1883, p. 46.

Zamenis taeniatus Cope

Ann. Rep. U. S. Nat. Mus., 1898 (1900), p. 815, fig. 182.

TyPe Locality: New Mexico west of Rio Grande.

RANGE: Idaho and Utah, southward to all the border states just entering Texas and California.

\section{Coluber taeniatus girardi STEJNEger and Barbour}

Coluber taeniatus girardi STEJNEGER and BARBOUR

Check List N. Amer. Amph. Rept., Ed. 1, 1917, p. 80.

Zamenis ornatus Cope

Ann. Rep. U. S. Nat. Mus., 1898 (1900), p. 813, fig. 181.

TYPE LOCALITy: “Between Indianola and El Paso," Texas. RANGE: Western Texas. 


\section{Salvadora ( $G E N U S)$}

Baird and Girard, Cat. N. Amer. Rept., Pt. 1, 1853, p. 104 TYPE: grahamiae

Salvadora grahamiae BAIRD and GIRARD PATCH-NOSE SNAKE

Salvadora grahamiae BAIRD and GIRARD

Cat. N. Amer. Rept., Pt. 1, 1853, p. 104.

Salvadora grahamiae (part) CoPE

Ann. Rep. U. S. Nat. Mus., 1898 (1900), p. 818.

TYPE LOCALITy: Sonora, Mexico.

RANGE: Utah southward through all the border states eastward of Arizona and into Mexico as far south as the State of Hidalgo.

\section{Salvadora hexalepis (CopE)}

Phimothyra hexalepis CoPE

Proc. Ac. Nat. Sci. Phila., 1866, p. 304.

Salvadora heralepis STEJNEgER and BARBour

Proc. U. S. Nat. Mus., Vol. 25, Sept. 8, 1902, p. 154.

Salvadora grahamiae (part) CopE

Ann. Rep. U. S. Nat. Mus., 1898 (1900), p. 818, fig. 183.

TYPE LOCALITY: Fort Whipple, Arizona.

RANGE: Arizona, southern California, and Lower California.

\section{Phyllorhynchus ( $G E N U S$ )}

Stejneger, Proc. U. S. Nat. Mus., Vol. 13, Sept. 9, 1890, p. 151 TYPE: browni

\section{Phyllorhynchus browni SteJneger}

Phyllorhynchus browni STEJNeger

Proc. U. S. Nat. Mus., Vol. 13, Sept. 9, 1890, p. 152.

Phyllorhynchus browni COPE

Ann. Rep. U. S. Nat. Mus., 1898 (1900), p. 821, fig. 184.

TyPe Locality: Tucson, Arizona.

RANGE: Southern Arizona.

\section{Phyllorhynchus decurtatus (CoPE)}

Phimothyra decurtata CoPE

Proc. Ac. Nat. Sci. Phila., 1868, p. 310.

Phyllorhynchus decurtatus SteJneger

Proc. U. S. Nat. Mus., Vol. 13, 1890, p. 154.

Phyllorhynchus decurtatus CoPE

Ann. Rep. U. S. Nat. Mus., 1898 (1900), p. 823, fig. 186. 
TYPE LOCALITY: "In the upper part of Lower California." RANGE: Lower California, north to southern California, southwestern Arizona and Nevada.

$$
\begin{aligned}
& \text { Elaphe (GENUS) } \\
& \text { Fitzinger in WAGLer, Deser. Icon. Amph., Pt. 3, 1833, } \\
& \text { text to plate } 27 \\
& \text { TYPE: parreysii = quatuorlineata }
\end{aligned}
$$

Elaphe bairdi (YARRow)

Coluber bairdi YARROW

Bull. U. S. Nat. Mus., No. 17, 1880, p. 41.

Elaphe bairdi Stejneger and Barbour

Check List N. Amer. Amph. Rept., Ed. 1, 1917, p. 82.

Coluber bairdii COPE

Ann. Rep. U. S. Nat. Mus., 1898 (1900), p. 854, fig. 198.

TyPe LOCAlity: Fort Davis, 50 miles northwest of Presidio, Texas.

RANGE: Known only from type locality and from Matagorda Co., Texas.

\section{Elaphe chlorosoma (GüNTHER)}

Coluber chlorosoma GüNTher

Biol. Centr.-Amer., Rept., 1894, p. 115, pl. 41.

Elaphe chlorosoma SteJNeger and Barbour

Check List N. Amer. Amph. Rept., Ed. 1, 1917, p. 82.

TYPE LOCALITY: Atoyak and Amula in Guerrero and San Ramon in Jalisco, Mexico.

RANGE: Guerrero and Jalisco, Mexico, northward to the Santa Rita Mountains in Arizona.

\section{Elaphe guttata (LINNÉ)}

\section{CORN-SNAKE}

Coluber guttatus LINNÉ

Syst. Nat., Ed. 12, Vol. 1, 1766, p. 385.

Elaphis guttatus Duméril and BibroN

Erp. Gén., Vol. 7, 1854, p. 273.

Coluber guttatus CoPE

Ann. Rep. U. S. Nat. Mus., 1898 (1900), p. 833, figs. 189, 190.

TYPE LOCALITY: Carolina.

RANGE: New Jersey to Florida, Louisiana, Missouri and Mississippi. 
Elaphe laeta (BAIRD and GIRARD)

Scotophis laetus BAIRD and GIRARD

Cat. N. Amer. Rept., Pt. 1, 1853, p. 77.

Elaphe laeta SteJneger and Barbour

Check List N. Amer. Amph. Rept., Ed. 1, 1917, p. 82.

Coluber laetus Cope

Ann. Rep. U. S. Nat. Mus., 1898 (1900), p. 850, fig. 196.

Coluber emoryi CoPE

Ann. Rep. U. S. Nat. Mus., 1898 (1900), p. 852, fig. 197.

TyPE LOCAlity: Red River, Arkansas.

RANGE: Kansas southward to Central Mexico.

Elaphe obsoleta obsoleta ( $\left.\mathrm{S}_{A X}\right)$

PILOT BLACK-SNAKE

Coluber obsoletus SAY

Long's Exp. Rocky Mts., Vol. 1, 1823, p. 140.

Elaphis obsoletus Garman

Mem. Mus. Comp. Zoöl., Vol. 8, No. 3, 1883, p. 54.

Coluber obsoletus obsoletus (part) COPE

Ann. Rep. U. S. Nat. Mus., 1898 (1900), p. 844, fig. 194.

TYPE LOCALity: Isle au Vache [sic] to Council Bluffs on the

Missouri River.

RANGE: Southern New England westward to Michigan, southward

to Texas, Louisiana, and Florida.

Elaphe obsoleta confinis (BAIRD and GIRARD)

Scotophis confinis BaIRD and GIRARD

Cat. N. Amer. Rept., Pt. 1, 1853, p. 76.

Elaphe obsoleta confinis StEJNEGER and Barbour

Check List N. Amer. Amph. Rept., Ed. 1, 1917, p. 83.

Coluber confinis Cope

Ann. Rep. U. S. Nat. Mus., 1898 (1900), p. 829, fig. 187.

TYPE LOCALITY: Anderson, South Carolina.

RANGE: South Atlantic and Gulf States and states of southern

Mississippi Valley.

Elaphe quadrivittata quadrivittata (HOLBROOK)

CHICKEN-SNAKE

Coluber quadrivittatus HоLвroоK

N. Amer. Herp., Ed. 1, Vol. 1, 1836, p. 113, pl. 21.

Coluber quadrivittatus CoPE

Ann. Rep. U. S. Nat. Mus., 1898 (1900), p. 838, fig. 192.

TYPE LOCALITY: "North Carolina to Florida and westward as

far as the Mississippi."

RANGE: Carolina to Florida and west to the Mississippi River. 


\section{Elaphe quadrivittata deckerti BraDY}

Elaphe quadrivittata deckerti Brady

Proc. Biol. Soc. Wash., Vol. 45, April 2, 1932, p. 5.

TYPE LOCAlity: Lower Matecumbe [sic] Key, Florida.

RANGE: The upper Florida Keys.

Elaphe rosacea (COPE)

Coluber rosaceus Cope

Proc. U. S. Nat. Mus., Vol. 11, 1888, p. 388, pl. 36, fig. 3.

Elaphe rosacea BARBour

Copeia, No. 84, July 31, 1920, p. 68.

TYPE LOCALITy: Key West, Florida.

raNGE: The lower Florida Keys.

Elaphe rosaliae (MocQuARD)

Coluber rosaliae MocQuARD

Nouv. Arch. Mus. Hist. Nat. Paris, Ser. 4, Vol. 1, 1899, p. 321, pl. 12, figs. 1-1b.

Elaphe rosaliae STEJNEger and BARBour

Check List N. Amer. Amph. Rept., Ed. 1, 1917, p. 84.

TYPE LOCALITY: Sta. Rosalia, Lower California.

RANGE: Central and southern Lower California.

Elaphe subocularis (Brown)

Coluber subocularis Brown

Proc. Ac. Nat. Sci. Phila., 1901, p. 492, pl. 29.

Elaphe subocularis STEJNEGER and Barbour

Check List N. Amer. Amph. Rept., Ed. 1, 1917, p. 84.

TYPE LoCality: Davis Mountains, Jeff Davis Co., 50 miles south of Pecos, Texas.

RANGe: Davis Mountains and Mt. Franklin, El Paso County, Texas.

Elaphe vulpina (BAIRD and GIRARD)

FOX-SNAKE

Scotophis vulpinus BAIRD and GiRARD

Cat. N. Amer. Rept., Pt. 1, 1853, p. 75.

Elaphe vulpinus Ruthven

Eleventh Rep. Mich. Ac. Sci., 1909, p. 116.

Coluber vulpinus CoPE

Ann. Rep. U. S. Nat. Mus., 1898 (1900), p. 831, fig. 188.

TYPE LOCA L ITY: Racine, Wisconsin.

RA NGE: Indiana, Iowa, and Illinois, to Michigan, Minnesota, and

Lake Superior. 


\section{Drymobius ( GENUS)}

Fimzinger, Syst. Rept., 1843, p. 26 TYPE: margaritiferus

\section{Drymobius margaritiferus (SCHLEGEL)}

Herpetodryas margaritiferus ScHLEGEL

Essai Physion. Serp., Vol. 2, 1837, p. 184.

Drymobius margaritiferus Cope

Proc. Ac. Nat. Sci. Phila., 1860, p. 561.

TYPE LOCALITY: New Orleans (in errore).

RANGE: Southwestern Texas to Venezuela and Colombia.

\section{Drymarchon ( $G E N U S$ )}

Fitzinger, Syst. Rept., 1843, p. 26. TYPE: corais

Drymarchon corais couperi (HoLBROOK) GOPHER-SNAKE, INDIGO-SNAKE

Coluber couperi HoLBrook

N. Amer. Herp., Ed. 2, Vol. 3, 1842, p. 75, pl. 16.

Drymarchon corais couperi STRECKER

Baylor Bulletin, Vol. 18, No. 4, Aug. 1915, p. 32.

Compsosoma corais couperii (part) Cope

Ann. Rep. U. S. Nat. Mus., 1898 (1900), p. 858, figs. 199, 200.

TYPE LOCALITY: Dry pine hills south of Alatamaha [sic],

Georgia.

RANGE: Carolinas to Florida, westward to Texas.

Drymarchon corais melanurus (DUMÉRIL and BIBRON)

Spilotes melanurus Duméril and BibroN

Erp. Gén., Vol. 7, 1854, p. 224.

Drymarchon corais melanurus STEJNEGER

N. Amer. Fauna, No. 14, 1899, p. 70.

TYPE LOCALITY: Mexico.

RANGE: Extreme southwestern Texas to northwestern South America.

\section{Arizona ( $G E N U S)$}

Kennicott in Baird, U. S. Mex. Bound. Surv., Vol. 2, 1859, Rept., p. 18 TYPE: elegans

Arizona elegans elegans (KENNICOTT)

Arizona elegans Kennicott

in Baird, U. S. Mex. Bound. Surv., Vol. 2, 1859, Rept., p. 18, pl. 13. 
Arizona elegans elegans BLANCHARD

Occ. Papers Mus. Zool. Univ. Mich., No. 150, April 28, 1924, p. 1.

Rhinechis elegans COPE

Ann. Rep. U. S. Nat. Mus., 1898 (1900), p. 863, fig. 201.

TYPE LOCALITY: Rio Grande, Texas, and between Arkansas and

Cimarron, Oklahoma.

RANGE: Border states of United States and northern states of

Mexico.

\section{Arizona elegans occidentalis BLANCHARD}

Arizona elegans occidentalis B Lanchard

Occ. Papers Mus. Zool. Univ. Mich., No. 150, April 26, 1924, p. 1. TYPE LOCALity: La Jolla, California.

RANGE: Southeastern Arizona, southern California and Lower California.

$$
\text { Pituophis }(G E N U S)
$$

Ноцввоок, N. Amer. Herp., Ed. 2, Vol. 4, 1842, p. 7 TYPE: melanoleucus

Pituophis catenifer catenifer (BLAINville) WESTERN BULL-SNAKE

Coluber catenifer Blainville

Nouv. Ann. Mus. Hist. Nat. Paris, Vol. 4, 1835, p. 290, pl. 26, figs. 2-2b.

Pituophis catenifer BAIRD and Girard

Cat. N. Amer. Rept., Pt. 1, 1853, p. 69.

Pituophis catenifer heermanni STEJNEGER and BaRBour

Check List N. Amer. Amph. Rept., Ed. 2, 1923, p. 95.

Pityophis catenifer COPE

Ann. Rep. U. S. Nat. Mus., 1898 (1900), p. 876, fig. 208.

TYPE LOCALITY: California.

RANGE: California to British Columbia.

Pituophis catenifer annectens (BAIRD and GIRARD)

Pituophis annectens BAIRD and Girard

Cat. N. Amer. Rept., Pt. 1, 1853, p. 72.

Pituophis catenifer annectens $\mathrm{V}_{\text {AN DeNBUrgh }}$

Proc. Calif. Ac. Sci., Ser. 4, Vol. 9, Aug. 21, 1919, p. 216.

TyPE LOCAlity: San Diego, California.

RANGE: Coast of southern and northern Lower California.

\section{Pituophis catenifer deserticola StejNeger}

Pituophis catenifer deserticola Stejneger

N. Amer. Fauna, No. 7, May 31, 1893, p. 206. 
Pituophis catenifer slejnegeri STEJNEGER and BARBour

Check List N. Amer. Amph. Rept., Ed. 2, 1923, p. 95.

TYPE LOCALITY: Great basin and southwestern deserts.

RANGE: Desert regions of southern California, Nevada and Utah, north to Idaho and eastern parts of Oregon and Washington.

Pituophis deppei deppei (Duméril and Bibron)

Elaphis deppei Duméril and Brbron

Erp. Gén., Vol. 7, 1854, p. 268.

Pituophis deppei deppei Stull

Occ. Papers Mus. Zool., Univ. Mich., No. 250, 1932, p. 1.

Epiglottophis pleurostictus Cope

Ann. Rep. U. S. Nat. Mus., 1898 (1900), p. 861.

TYPE LOCALITY: Mexico.

RAnge: Hay's County, Texas and Real de Pinos, California to southern Oaxaca, Mexico.

\section{Pituophis lödingi Blanchard}

Pituophis lödingi BLANCHARD

Papers Mich. Acad. Sci. Arts Letters, Vol. 4, 1924, p. 531.

type locality: Grand Bay, Mobile Co., Alabama.

RANGE: Southern Alabama.

Pituophis melanoleucus (Daudin)

PINE-SNAKE

Coluber melanoleucus Daudin

Hist. Nat. Rept., Vol. 6, 1803, p. 409.

Pituophis melanoleucus HoLbrook

N. Amer. Herp., Ed. 2, Vol. 4, 1842, p. 7, pl. 1.

Pituophis melanoleucus melanoleucus BarBour

Proc. N. Engl. Zoöl. Club, Vol. 7, Oct. 22, 1921, p. 117.

Pityophis melanoleucus CopE

Ann. Rep. U. S. Nat. Mus., 1898 (1900), p. 867, fig. 203.

TYPE LOCALITY: South Carolina and Florida.

RANGE: Pine barrens of southern New Jersey to South Carolina, west to Tennessee.

\section{Pituophis mugitus ${ }^{1}$ (BARBOUR)}

Pituophis melanoleucus mugitus BARBOUR

Proc. N. Engl. Zoöl. Club, Vol. 7, Oct. 22, 1921, p. 117.

ty RANGE: Peninsular Florida.

1 This species, the preceding, and the following may be subspecies, but they are very different and their respective ranges may not meet. 
Pituophis ruthveni (STULL)

Pituophis melanoleucus ruthveni STULL

Occ. Papers Mus. Zool. Univ. Mich., No. 205, June 19, 1929, p. 1. TyPe lOCAlity: Longleaf, Rapides County, Louisiana. RANGE: Type locality.

Pituophis sayi sayi (SchlegeL)

BULL-SNAKE

Coluber sayi Schlegel

Essai Physion. Serp., Pt. 2, 1837, p. 157.

Pityophis sayi sayi COPE

Ann. Rep. U. S. Nat. Mus., 1898 (1900), p. 870, fig. 204.

TYPE LOCALITY: Missouri.

RANGE: Northern Mexico and Texas north to Wisconsin and Alberta.

\section{Pituophis sayi affinis (HALLOWELL)}

Pituophis affinis Hallowel.

Proc. Ac. Nat. Sci. Phila., 1852, p. 181.

Pituophis sayi affinis STULL

Occ. Papers Mus. Zool. Univ. Mich., No. 250, Oct. 19, 1939, p. 4.

Pituophis catenifer rutilus Van Denburgh

Proc. Calif. Ac. Sci., Ser. 4, Vol. 10, Aug. 6, 1920, p. 24, pl. 2, fig. 2. Pityophis sayi bellona CopE

Ann. Rep. U. S. Nat. Mus., 1898 (1900), p. 872 (part).

TyPE LOCALity: New Mexico.

RANGE: Southern Colorado south in Chihuahua, Mexico; west through Arizona and southern California and south to Colorado Desert, Lower California.

\section{Pitucphis vertebralis (BLAINviLle)}

Coluber vertebralis BLAINviLLE

Nouv. Ann. Mus. Hist. Nat. Paris, Vol. 4, 1835, p. 293, pl. 27, figs. 2-2b.

Pituophis vertebralis DuMÉrIL and BIBRON

Erp. Gén., Vol. 7, 1854, p. 238.

Pityophis vertebralis CoPE

Ann. Rep. U. S. Nat. Mus., 1898 (1900), p. 879, fig. 209.

TYPE LOCALITY: California, a term which formerly included Lower California.

RANGE: Throughout Lower California, as far north into California as Jim Grey, San Bernardino County. It occurs also on the outlying islands of Cerros, Margarita and Magdalena. 


\section{Rhadinaea ( $G E N U S)$}

Cope, Proc. Ac. Nat. Sci. Phila., 1863, p. $101 .^{1}$

TYPE : vermiculaticeps

\section{Rhadinaea flavilata (COPE)}

Dromicus flavilatus Cope

Proc. Ac. Nat. Sci. Phila., 1871, p. 292.

Leimadophis flavilatus STEJNEGER and BARBOUR

Check List N. Amer. Amph. Rept., Ed. 1, 1917, p. 86, Ed 2, 1923, p. 96.

Rhadinaea flavilata Cope

Ann. Rep. U. S. Nat. Mus., 1898 (1900), p. 759, fig. 164.

TyPE LOCALITy: Fort Macon, North Carolina.

RANGE: The Carolinas to Florida.

\section{Lampropeltis ( $G E N U S$ )}

Fitzinger, Syst. Rept., 1843, p. 25

TYPE: getulus Schlegel, nec LINNÉ = holbrooki

\section{Lampropeltis alterna (BRown)}

Ophibolus alternus Brown

Proc. Ac. Nat. Sci. Phila., 1901 (Feb. 6, 1902), p. 612.

Lampropeltis alterna STEJNEGER and BARBOUR

Check List N. Amer. Amph. Rept., Ed. 1, 1917, p. 87.

ty PE Locality: Davis Mountains, Jeff Davis Co., Texas.

RANGE: Davis Mountains, Texas.

\section{Lampropeltis californiae californiae (BLAINVILLE)}

Coluber (Ophis) californiae BLAINville

Nouv. Ann. Mus. Hist. Nat. Paris, Vol. 4, 1835, p. 292, pl. 27, figs. $1-1 b$.

Lampropeltis californiae californiae BLANCHARD

Occ. Papers Mus. Zool. Univ. Michigan, No. 87, 1920, p. 3.

Ophibolus getulus californiae CoPE

Ann. Rep. U. S. Nat. Mus., 1898 (1900), p. 922, fig. 231.

TYPE LOCALITY: California.

RANGE: Southern California.

\section{Lampropeltis californiae nitida (VAN DENBURGh)}

Lampropeltis nitida VAN DeNBURGH

Proc. Calif. Ac. Sci., Ser. 2, Vol. 5, 1895, p. 143, pl. 14.

Lampropeltis californiae nitida BLANCHARD

Occ. Papers Mus. Zool. Univ. Michigan, No. 87, 1920, p. 3.

1 Dunn has the date of the appearance of this generic name incorrectly cited as 1860. 
Ty PE locality: San José del Cabo, Lower California.

RANGE: Southern Lower California.

Lampropeltis calligaster (HARLAN)

Coluber calligaster HARLAN

Journ. Ac. Nat. Sci. Phila., Vol. 5, 1827, p. 359.

Lampropeltis calligaster COPE

Proc. Ac. Nat. Sci. Phila., 1860, p. 255.

Ophibolus calligaster COPE

Ann. Rep. U. S. Nat. Mus., 1898 (1900), p. 905, fig. 223.

TYPE LOCALITY: Missouri.

RANGE: Indiana, Illinois and Wisconsin; Mississippi, Kansas,

Oklahoma and Texas.

Lampropeltis catalinensis VAN Denburgh and Slevin

Lampropeltis catalinensis $\mathrm{V}_{\mathrm{AN}}$ Denburgh and SLevin

Proc. Calif. Ac. Sci., Ser. 4, Vol. 11, Dec. 17, 1921, p. 397.

TYPE LOCALity: Santa Catalina Island, Gulf of California.

R ANGE: Same.

\section{Lampropeltis elapsoides elapsoides (НоLBROoK)}

Coluber elapsoides HoLBrook

N. Amer. Herp., Ed. 1, Vol. 2, 1838, p. 123, pl. 28.

Lampropeltis elapsoides Stejneger and Barbour

Check List N. Amer. Amph. Rept., Ed. 1, 1917, p. 88.

Osceola elapsoidea CoPE

Ann. Rep. U. S. Nat. Mus., 1898 (1900), p. 900, fig. 221.

TYPE LOCALITY: South Carolina and Georgia.

RANGE: Southeastern states to Florida and Louisiana.

Lampropeltis elapsoides virginiana BLANCIIARD

Lampropeltis elapsoides virginiana BLANCHARD

Occ. Papers Mus. Zool. Univ. Michigan, No. 81, Apr. 28, 1920, p. 2. ty Pe locality: Raleigh, North Carolina.

RANGE: Northern North Carolina and Virginia, east of the Allegheny Mountains.

Lampropeltis getulus getulus (LiNNÉ)

KING-SNAKE, CHAIN-SNAKE

Coluber getulus LiNNÉ

Syst. Nat., Ed. 12, Vol. 1, 1766, p. 382.

Lampropeltis getula CoPE

Proc. Ac. Nat. Sci. Phila., 1860, p. 255.

Ophibolus getulus getulus COPE

Ann. Rep. U. S. Nat. Mus., 1898 (1900), p. 914, figs. 227, 228. 
TYPE LOCALITY: Carolina.

RANGE: Southern New England to northern Florida.

Lampropeltis getulus boylii (BAIRD and GIRARD)

Ophibolus boylii BAIRD and GIRARD

Cat. N. Amer. Rept., Pt. 1, 1853, p. 82.

Lampropeltis getulus boylii BLANCHARD

Occ. Papers Mus. Zool. Univ. Michigan, No. 87, 1920, p. 3.

Ophibolus getulus boylii COPE

Ann. Rep. U. S. Nat. Mus., 1898 (1900), p. 919, fig. 230.

TYPE LoCAlity: El Dorado County, California.

RANGE: Arizona, western Nevada, California.

Lampropeltis getulus brooksi BARBOUR

Lampropeltis getulus brooksi $\mathrm{B}_{\mathrm{ARBOUR}}$

Proc. N. Engl. Zoöl. Club, Vol. 7, June 5, 1919, p. 1, pl. 1.

TYPE LOCAlity: Royal Palm Hammock, 14 miles southwest of

Homestead, Dade Co., southeastern Florida.

RANGE: The tropical southeastern corner of Dade County,

Florida.

\section{Lampropeltis getulus conjuncta (COPE)}

Lampropeltis boylii var. conjuncta CoPE

Proc. Ac. Nat. Sci. Phila., 1861, p. 301.

Lampropeltis getulus conjuncta BLANCHARD

Occ. Papers Mus. Zool. Univ. Michigan, No. 87,1920, p. 4.

TyPE LOCALITy: Cape St. Lucas, Lower California.

RANGE: Southwestern Arizona and Lower California.

\section{Lampropeltis getulus floridana BLANCHARD}

Lampropeltis getulus floridana BLANCHARD

Occ. Papers Mus. Zool. Univ. Michigan, No. 70, May 5, 1919, p. 1, pl. 1, fig. 1 .

TYPE Locality: Orange Hammock, De Soto Co., Florida.

RANGE: Florida from Orange County in the north to central Dade

County in the southeast.

\section{Lampropeltis getulus holbrooki (STEJNEGER)}

Lampropeltis holbrooki STEJNEGER

Proc. U. S. Nat. Mus., Vol. 25, Sept. 8, 1902, p. 152.

Lampropeltis getulus holbrooki HURTER and STRECKER

Trans. Ac. Sci. St. Louis, Vol. 18, 1909, p. 26.

Ophibolus getulus sayi Cope

Ann. Rep. U. S. Nat. Mus., 1898 (1900), p. 911, fig. 226. 
TYPE LOCALITY: Valley of the Mississippi.

RANGE: Louisiana to Texas, northward through the states of the Mississippi basin mostly west of the river, to southern Iowa and southern Nebraska.

Lampropeltis getulus nigra (YARROW)

Ophibolus getulus niger YARROW

Proc. U. S. Nat. Mus., Vol. 5, 1882, p. 438.

Lampropeltis getulus niger BLANCHARD

Occ. Papers Zool. Mus. Univ. Michigan, No. 87,1920 p. 2.

TYPE LOCALITY: Wheatland, Indiana.

RANGE: Northern Alabama, Tennessee, West Virginia, Kentucky, Ohio and southern Indiana.

\section{Lampropeltis getulus splendida (BAIRD and GIRARD)}

Ophibolus splendidus BaIRD and GIRARD

Cat. N. Amer. Rept., Pt. 1, 1853, p. 83.

Lampropeltis splendida COPE

Proc. Ac. Nat. Sci. Phila., 1860, p. 255.

Lampropeltis getulus splendida BLANCHARD

Occ. Papers Zool. MLus. Univ. Michigan, No. 87,1920 p. 2.

Ophibolus getulus splendidus COPE

Ann. Rep. U. S. Nat. Mus., 1898 (1900), p. 918, fig. 299.

TYPE LOCALITY: Sonora, Mexico.

RANGE: Northern Mexico and the boundary region.

\section{Lampropeltis getulus yumensis BLANCHARD}

Lampropeltis getulus yumensis BLANCHARD

Occ. Papers Mus. Zool. Univ. Michigan, No. 70, 1919, p. 6, pl. 1, fig. 2.

Lampropeltis getulus yumanensis STEJNEGER and BARBOUR

Check List N. Amer. Amph. Rept., Ed. 2, 1923, p. 100.

ty PE Locality: 27 miles west of Indian Oasis, Pima Co., Arizona.

RANGE: Southwestern Arizona, southeastern California, and adjacent areas of Sonora and Lower California.

\section{Lampropeltis multicincta (YARROW)}

Ophibolus getulus multicinctus $\mathrm{YARROW}_{\mathrm{A}}$

Proc. U. S. Nat. Mus., Vol. 5, 1882, p. 440.

Lampropeltis multicincta BLANCHARD

Occ. Papers Zool. Mus. Univ. Michigan, No. 87, 1920, p. 5.

Lampropeltis pyromelaena multicincta STEJNEGER and BaRBouR

Check Jist N. Amer. Amph. Rept., Ed. 1, 1917, p. 89.

Lampropeltis agalma STEJNEGER and BARBOUR

Check List N. Amer. Amph. Rept., Ed. 2,1923, p. 97. 
Lampropeltis herrerae STEJNEGER and BARBOUR

Check List N. Amer. Amph. Rept., Ed. 2, 1923, p. 100.

TYPE LOCALITY: Fresno, California.

RANGE: Moist coniferous forests of California, and Lower California.

\section{Lampropeltis pyromelana (COPE)}

Ophibolus pyromelanus COPE

Proc. Ac. Nat. Sci. Phila., 1866, p. 305.

Lampropeltis pyrrhomelaena STEJNEGER

Proc. U. S. Nat. Mus., Vol. 25, 1902, p. 152.

Ophibolus pyrrhomelas Cope

Ann. Rep. U. S. Nat. Mus., 1898 (1900), p. 907, fig. 224.

TYPE LOCALIT : Fort Whipple, Arizona.

RANGE: Southern Arizona.

\section{Lampropeltis rhombomaculata (HoLBRook)}

MOLE-SNAKE

Coluber rhombomaculatus HoLBRoок

N. Amer. Herp., Ed. 1, Vol. 4, 1840, p. 103, pl. 20.

Lampropeltis rhombomaculata $\mathrm{COPE}$

Proc. Ac. Nat. Sci. Phila., 1860, p. 255.

Ophibolus rhombomaculatus COPE

Ann. Rep. U. S. Nat. Mus., 1898 (1900), p. 903, fig. 222.

TYPE LOCALITY: Georgia and Alabama.

RANGE: Maryland to Georgia and Alabama.

\section{Lampropeltis triangulum triangulum (LACÉPÈDE)}

\section{MILK-SNAKE}

Coluber triangulum LACÉPÈDE

Hist. Nat. Quadr. Ovip. Serp., Vol. 2, 1788, tabl. méth., p. 86.

Lampropeltis triangula CoPE

Proc. Ac. Nat. Sci. Phila., 1860, p. 256.

Osceola doliata triangula COPE

Ann. Rep. U. S. Nat. Mus., 1898 (1900), p. 885, figs. 210-215.

TYPE LOCALITY: America.

RANGE: Eastern North America from Maine through Ontario to

Iowa, south to Virginia and in the mountains farther south.

\section{Lampropeltis triangulum amaura (COPE)}

Lampropeltis amaura COPE

Proc. Ac. Nat. Sci. Phila., 1860, p. 258.

Lampropeltis triangulum amaura STEJNEGER and BARBOUR

Check List N. Amer. Amph. Rept., Ed. 1, 1917, p. 90. 
Osceola doliata coccinea CoPE

Ann. Rep. U. S. Nat. Mus., 1898 (1900), p. 896, fig. 220 (not of Schlegel).

TYPE LOCALITY: “Unknown” (original entry of type U. S. Nat. Mus. No. 5282 reads "?Mississippi”).

RANGE: Eastern United States, west to Texas, south to the Gulf.

\section{Lampropeltis triangulum annulata (KENNICOTT)}

Lampropeltis annulata KENNICOTT

Proc. Ac. Nat. Sci. Phila., 1860, p. 329.

Lampropeltis triangulum annulata BLANCHARD

Occ. Papers Zool. Mus. Univ. Michigan, No. 87, 1920, p. 5.

Osceola doliata annulata COPE

Ann. Rep. U. S. Nat. Mus., 1898 (1900), p. 895, fig. 219.

TYPE LOCALITY: Matamoros, Mexico.

RANGE: Southeastern Texas and northeastern Mexico.

Lampropeltis triangulum gentilis (BAIRD and GIRARD)

Ophibolis gentilis BaIRD and Girard

Cat. N. Amer. Rept., Pt. 1, 1853, p. 90.

Lampropeltis triangulum gentilis STEJNEGER and $\mathrm{B}_{\mathrm{ARBOUR}}$

Check List N. Amer. Amph. Rept., Ed. 1, 1917, p. 90.

Osceola doliata gentilis Cope

Ann. Rep. U. S. Nat. Mus., 1898 (1900), p. 894, fig. 218.

Lampropeltis pyromelaena celaenops STEJNEGER and BARBOUR

Check List N. Amer. Amph. Rept., Ed. 1, 1917, p. 89.

TyPE Locality: North Fork, Red River, near Sweetwater Creek, Wheeler Co., Texas.

RANGE: Texas and northwestern Mexico; Kansas and Utah.

\section{Lampropeltis triangulum syspila (COPE)}

Ophibolus doliatus syspilus Cope

Proc. U. S. Nat. Mus., Vol. 11, 1888, p. 384.

Lampropeltis triangulum syspila RuthveN

Occ. Papers Mus. Zool. Univ. Michigan, No. 66, 1919, p. 3.

Osceola doliata syspila CoPE

Ann. Rep. U. S. Nat. Mus., 1898 (1900), p. 891, fig. 216.

TYPE LOCALITY: Richland County, Illinois.

RANGE: Oklahoma, Arkansas and Tennessee northward to Kansas, Iowa, Illinois and Indiana. 


\author{
Stilosoma ( GENUS) \\ Brown, Proc. Ac. Sci. Phila., 1890, p. 199 \\ TYPE: extenuatum
}

\title{
Stilosoma extenuatum Brown
}

Stilosoma extenuatum Brown

Proc. Ac. Nat. Sci. Phila., 1890, p. 199.

Stylophis extenuatus STEJNEGER and BARBour

Check List N. Amer. Amph. Rept., Ed. 1, 1917, p. 90.

Stilosoma extenuatum CoPE

Ann. Rep. U. S. Nat. Mus., 1898 (1900), p. 924, fig. 232.

TYPE LOCAlity: Lake Kerr, Marion Co., Florida.

RANGE: North Central Florida.

\section{Contia ( $G E N U S)$}

Baird and Girard, Cat. N. Amer. Rept., Pt. 1, 1853, p. 110 TYPE: mitis $=$ tenuis

Contia tenuis (Baird and Girard)

Calamaria tenuis BAIRD and GIRARD

Proc. Ac. Nat. Sci. Phila., Vol. 6, 1852, p. 176.

Contia tenuis STEJNEger and Barbour

Check List N. Amer. Amph. Rept., Ed. 1, 1917, p. 91.

Contia mitis Cope

Ann. Rep. U. S. Nat. Mus., 1898 (1900), p. 925, fig. 233.

Lodia tenuis COPE

Ann. Rep. U. S. Nat. Mus., 1898 (1900), p. 927, fig. 234.

TYPE LOCALITy: Puget Sound, Washington.

RANGe: Southern California to Puget Sound.

\section{Cemophora ( $G E N U S)$}

Cope, Proc. Ac. Nat. Sci. Phila., 1860, p. 244

TYPE: coccinea

\section{Cemophora coccinea (Blumenbach)}

Coluber coccineus BLUMENBACH

Voigt's Mag. Neu. Phys. Naturg., Vol. 5, Pt. 1, 1788, p. 11, pl. 1.

Cemophora coccinea CoPE

Proc. Ac. Nat. Sci. Phila., 1860, p. 244.

Cemophora coccinea CoPE

Ann. Rep. U. S. Nat. Mus., 1898 (1900), p. 928, fig. 235.

TYPE LOCALITY: Florida.

RANGE: Maryland to Alabama, Louisiana, Oklahoma and Florida. 


\section{Rhinocheilus (GENUS)}

Baird and Girard, Cat. N. Amer. Rept., Pt. 1, 1853, p. 120

$$
\text { TYPE: leconte } i
$$

Rhinocheilus lecontei BAIRD and GIRARD

Rhinochcilus lecontei BAIRD and GIRARD

Cat. N. Amer. Rept., Pt. 1, 1853, p. 120.

Rhinochilus lecontei CopE

Ann. Rep. U. S. Nat. Mus., 1898 (1900), p. 931, fig. 236.

TyPe Locality: San Diego, California.

RANGE: Idaho to Lower California, east to Texas, northern

Mexico.

\section{Sonora (GENUS)}

Baird and Girard, Cat. N. Amer. Rept., Pt. 1, 1853, p. 117 TYPE: semiannulata

\section{Sonora occipitalis (HALLOWELL)}

Rhinostoma occipitale HALLOWELL

Proc. Ac. Nat. Sci. Phila., Vol. 7, 1854, p. 95.

Sonora occipitalis Van Denburgh

Proc. Calif. Ac. Sci., Ser. 4, Vol. 3, 1913, p. 411.

Chionactis occipitalis COPE

Ann. Rep. U. S. Nat. Mus., 1898 (1900), p. 941, fig. 240.

TYPE LOCALITy: Mojave Desert.

RANGE: Southeastern California, Arizona, Colorado.

\section{Sonora semiannulata BAIRD and GIRARD}

Sonora semiannulata $\mathrm{B}_{\mathrm{AIRD}}$ and GIRARD

Cat. N. Amer. Rept., Pt. 1, 1853, p. 117.

Chionactis episcopus isozonus CoPE

Ann. Rep. U. S. Nat. Mus., 1898 (1900), p. 939, fig. 239.

TyPE LOCALITy: Sonora, Mexico.

RANGE: Idaho to Utah and Nevada, Arizona, southern Kansas and Texas; also southeastern California and the adjacent regions of Mexico.

\section{Sonora taylori (BOULENGER)}

Contia taylori Boulenger

Cat. Snakes Brit. Mus., Vol. 2, 1894, pl. 12, fig. 3.

Sonora taylori STEJNEger and Barbour

Check List N. Amer. Amph. Rept., Ed. 1, 1917, p. 92.

Chionactis taylorii Cope

Ann. Rep. U. S. Nat. Mus., 1898 (1900), p. 936. 
TyPe locality: Duval County, Texas, and Nuevo Leon, Mexico.

RANGE: Southern Texas and northeastern Mexico.

\author{
Ficimia ( $G E N U S)$ \\ GraY, Cat. Snakes Brit. Mus., 1849, p. 80 \\ TYPE: olivacea
}

Ficimia cana (Cope)

Gyalopion canum CoPE

Proc. Ac. Nat. Sci. Phila., 1860, p. 243.

Ficimia cana GARMaN

Mem. Mus. Comp. Zoöl., Vol. 8, Pt. 3, 1883, p. 83.

Gyalopium canum Cope

Ann. Rep. U. S. Nat. Mus., 1898 (1900), p. 947, fig. 241.

TYPE LOCALITY: Fort Buchanan, Arizona.

RANGE: Western Texas to Arizona.

Ficimia streckeri TAYLOR

Ficimia streckeri TAYLOR

Copeia, 1931, No. 1, March 31, p. 5.

TyPe LOCALIty: Three miles east of Rio Grande City, Texas.

RANGE: Same as type locality.

\title{
Hypsiglena ( $G E N U S$ )
}

Cope, Proc. Ac. Nat. Sci. Phila., 1860, p. 246

TYPE: ochrorhynchus

\section{Hypsiglena ochrorhynchus Cope}

Hypsiglena ochrorhynchus COPE

Proc. Ac. Nat. Sci. Phila., 1860, p. 246.

Hypsiglena ochrorhynchus texana STENNEGER and BARBOUR

Check List N. Amer. Amph. Rept., Ed. 2, 1923, p. 105.

Hypsiglena ochrorhyncha COPE

Ann. Rep. U. S. Nat. Mus., 1898 (1900), p. 953, fig. 245. tyPE LOCAlity: Cape St. Lucas, Lower California.

RANGE: Southern and Lower California, Arizona, southern Utah,

Idaho, southern Nevada, southwestern Texas, and northwestern Mexico. 


\title{
Chilomeniscus ( $G$ ENUS) \\ Cope, Proc. Ac. Nat. Sci. Phila., 1860, p. 339 \\ TYPE: stramineus
}

Chilomeniscus cinctus COPE

Chilomeniscus cinctus CoPE

Proc. Ac. Nat. Sci. Phila., 1861, p. 303.

TYPE LOCALITY: Guaymas, Mexico.

RANGE: Colorado Desert to adjacent Sonora and central Lower California.

Chilomeniscus punctatissimus Van Denburgh and Slevin

Chilomeniscus punctatissimus VAN Denburgh and Slevin

Proc. Calif. Ac. Sci., Ser. 4, Vol. 11, July 30, 1921, p. 98.

type locality: Isla Partida, Espiritu Santo Island, Lower

California.

RANGE: Same.

\section{Chilomeniscus stramineus CoPE}

Chilomeniscus stramineus CopE

Proc. Ac. Nat. Sci. Phila., 1860, p. 339.

Chilomeniscus stramineus COPE

Ann. Rep. U. S. Nat. Mus., 1898 (1900), p. 949, fig. 242.

TyPe locality: Cape St. Lucas, Lower California.

RANGE: Southern Lower California.

\author{
Natrix (GENUS) \\ Laurenti, Syn. Rept., 1768, p. 73 \\ TYPE: vulgaris $=$ natrix
}

Natrix clarkii (BAIRD and Girard)

Regina clarkii BAIRD and GIRARD

Cat. N. Amer. Rept., Pt. 1, 1853, p. 48.

Natrix clarkii Cope

Proc. U. S. Nat. Mus., Vol. 14, 1892, p. 669.

Natrix clarkii Cope

Ann. Rep. U. S. Nat. Mus., 1898 (1900), p. 987, fig. 261. TYPE LOCALITY: Indianola, Texas.

RANGE: Coastal regions of northwest Florida to Texas. 
Natrix compressicauda (KENNICOTT)

SALT-WATER MOCCASIN

Nerodia compressicauda KenNICOTT

Proc. Ac. Nat. Sci. Phila., 1860, p. 335.

Natrix compressicauda COPE

Proc. U. S. Nat. Mus., Vol. 11, 1889, p. 392.

Natrix compressicauda CoPE

Ann. Rep. U. S. Nat. Mus., 1898 (1900), p. 978, fig. 255.

TYPE LoCALITy: Tampa Bay, Florida.

RANGE: Southwestern Florida, especially the mangrove swamps

of the extreme southern coast and the Keys.

Natrix cyclopion (DumÉrIL and BIBRoN)

Tropidonotus cyclopion Duméril and BibroN

Erp. Gén., Vol. 7, 1854, p. 576.

Natrix cyclopium CoPE

Proc. U. S. Nat. Mus., Vol. 14, 1892, p. 673.

Natrix cyclopium CoPE

Ann. Rep. U. S. Nat. Mus., 1898 (1900), p. 961, figs. 247, 248.

TYPE LOCALity: New Orleans.

RANGE: Florida to Louisiana and northward up the Mississippi

Valley to southern Illinois.

Natrix grahamii (BAIRD and GiRARD)

Regina grahamii BAIRD and GIRARD

Cat. N. Amer. Rept., Pt. 1, 1853, p. 47.

Natrix grahamii CoPE

Proc. U. S. Nat. Mus., Vol. 14, 1892, p. 668.

Natrix grahamii CoPE

Ann. Rep. U. S. Nat. Mus., 1898 (1900), p. 991, fig. 264.

TYPE LOCALITY: Rio Salado, Texas.

RANGE: Illinois southward to Louisiana and Texas.

Natrix kirtlandii (KeNNICOTT)

Regina kirtlandii KenNicotT

Proc. Ac. Nat. Sci. Phila., 1856, p. 95.

Natrix kirtlandii COPE

Ann. Rep. U. S. Nat. Mus., 1898 (1900), p. 995, fig. 266.

TYPE LOCALITY: Northern Illinois.

RANGe: Michigan, Wisconsin, Illinois, Indiana, Ohio, Pennsylvania and New Jersey.

\section{Natrix rhombifera (HALLOWELL)}

Tropidonotus rhombifer HALLOWELL

Proc. Ac. Nat. Sci. Phila., Vol. 6, 1852, p. 177. 
Natrix rhombifera Cope

Proc. U. S. Nat. Mus., Vol. 11, 1889, p. 398.

Natrix rhombifera COPE

Ann. Rep. U. S. Nat. Mus., 1898 (1900), p. 963, fig. 249.

TYPE LOCALITY: Arkansas River and its tributaries near the northern boundary of the Creek Nation.

RANGE: Southern Illinois and Indiana south through Texas to Vera Cruz, Mexico.

Natrix rigida ( $\mathrm{SAY}_{\mathrm{A}}$

Coluber rigidus $\mathrm{S}_{\mathrm{AY}}$

Journ. Ac. Nat. Sci. Phila., Vol. 4, Pt. 2, 1825, p. 239.

Natrix rigida Cope

Proc. U. S. Nat. MLus., Vol. 14, 1892, p. 668.

Natrix rigida Cope

Ann. Rep. U. S. Nat. Mus., 1898 (1900), p. 989, fig. 262.

TYPE LOCALITY: The Southern States.

RANGE: The Carolinas to Louisiana.

Natrix septemvittata $\left(\mathrm{S}_{\mathrm{AY}}\right)$

MOON-SNAKE, QUEEN-SNAKE

Coluber septemvittatus $\mathrm{S}_{\mathrm{AY}}$

Journ. Ac. Nat. Sci. Phila., Vol. 4, Pt. 2, 1825, p. 240.

Natrix septemvittata Cope

Trans. Amer. Philos. Soc., Vol. 18, 1895, p. 216.

Natrix leberis Cope

Ann. Rep. U. S. Nat. Mus., 1898 (1900), p. 993, fig. 265.

TyPe locality: Pennsylvania.

RANGe: Central, Eastern and Gulf States from Pennsylvania, Ohio, and Wisconsin southward.

Natrix sipedon sipedon (LINNÉ)

WATER-SNAKE

Coluber sipedon LinNÉ

Syst. Nat., Ed.'10, Vol. 1, 1758, p. 219.

Natrix sipedon Kinsch

Bull. U. S. Fish Comm., Vol. 14, 1895, p. 333.

Natrix fasciata sipedon CopE

Ann. Rep. U. S. Nat. Mus., 1898 (1900), p. 969, fig. 251.

TyPE Locality: North America.

RANGE: Southern Maine west to Minnesota and Colorado, south to northern Alabama, Arkansas and Oklahoma. 
Natrix sipedon confluens (BLANCHARD)

Natrix fasciata confluens Blanchard

Occ. Papers Mus. Zool. Univ. Michigan, No. 140, July 19, 1923, p. 1. Ty PE LOCALITy: Butler County, Missouri.

RANGE: Eastern Louisiana north through southern and eastern Arkansas to southeastern Missouri and west to Texas to about the 98th Meridian.

\section{Natrix sipedon fasciata ${ }^{1}$ (LINNÉ)}

Coluber fasciatus LinNÉ

Syst. Nat., Ed. 12, Vol. 1, 1766, p. 378.

Natrix sipedon fasciata SteJNEger and BARBour

Check List N. Amer. Amph. Rept., Ed. 1, 1917, p. 96.

Natrix fasciata fasciata CopE

Ann. Rep. U. S. Nat. Mus., 1898 (1900), p. 966, fig. 250.

TYPE LOCALITY: Carolina.

RANGE: Virginia to Florida, westward to Louisiana and northward up the Mississippi through all the central states to Nebraska and Indiana.

\section{Natrix sipedon pictiventris (COPE)}

Natrix fasciata pictiventris CoPE

Amer. Nat., Vol. 29, 1895, p. 677.

Natrix sipedon pictiventris STEJNEGER and BARBOUR

Check List N. Amer. Amph. Rept., Ed. 1, 1917, p. 96.

Natrix fasciata pictiventris Cope

Ann. Rep. U. S. Nat. Mus., 1898 (1900), p. 969.

TYPE LOCALity: Palatka, Florida.

RANGE: Peninsula of Florida.

\section{Natrix taxispilota (HoLBRooK)}

\section{WATER-PILOT}

Tropidonotus taxispilotus HoLBRooK

N. Amer. Herp., Ed. 2, Vol. 4, 1842, p. 35, pl. 8.

Natrix taxispilota COPE

Proc. U. S. Nat. Mus., Vol. 11, 1889, p. 392.

Natrix taxispilota Cope

Ann. Rep. U. S. Nat. Mus., 1898 (1900), p. 959, fig. 246.

TYPE LOCALITY: South Carolina seaboard and the Altamaha

River, Georgia.

RANGE: Virginia, the Carolinas to Florida, west to Louisiana.

1 Apparent forms which are more or less uniformly rufescent are found in the ranges of both the subspecies fusciata and sipedon. To these the namcs of Natrix sipedon erythrogaster (Forster in Bossu, Travels through North America, Vol. 1, 1771, p. 364, footnote) and Natrix sipedon fulviventer (Rafinesque, Ann. Nat., 1820, No. 1, p. 6) have been given. 
Natrix transversa (HaLloweld)

Tropidonotus transversus HaLlowell

Proc. Ac. Nat. Sci. Phila., Vol. 6, 1852, p. 177.

Natrix sipedon transversa Hurter

Herp. Missouri, 1911, p. 157.

Natrix fasciata transversa CoPE

Ann. Rep. U. S. Nat. Mus., 1898 (1900), p. 973, fig. 252.

TYPE LOCALITY: Creek boundary, found near the banks of the

Arkansas and its tributaries.

RANGE: Kansas, Arkansas, Oklahoma, and Texas, south into

Mexico and west to New Mexico.

Natrix valida (KenNicotT)

Regina valida KenNicotT

Proc. Ac. Nat. Sci. Phila., 1860, p. 334.

Natrix valida Cope

Proc. U. S. Nat. Mus., Vol. 14, 1892, p. 670.

Natrix valida Cope

Ann. Rep. U. S. Nat. Mus., 1898 (1900), p. 984, figs. 258, 259.

TyPe LOCality: Durango, Mexico.

RANGE: Western Mexico, Lower California.

Seminatrix ( $G E N U S)$

Cope, Amer. Nat., Vol. 29, 1895, p. 678

TYPE: pygaea

\section{Seminatrix pygaea (COPE)}

Contia pygaea CoPE

Proc. Ac. Nat. Sci. Phila., 1871, p. 223.

Seminatrix pygaea CoPE

Amer. Nat., Vol. 29, 1895, p. 678.

Seminatrix pygaea CoPE

Ann. Rep. U. S. Nat. Mus., 1898 (1900), p. 998, fig. 267.

TYPE LOCALity: Volusia, Florida.

RANGE: South Carolina south through Florida peninsula.

\section{Storeria ( $G E N U S$ )}

Baird and Girard, Cat. N. Amer. Rept., Pt. 1, 1853, p. 135 TYPE: dekayi

\section{Storeria dekayi (НоLвRоoк)}

DEKAY'S SNAKE

Tropidonotus dekayi HoLBrook

N. Amer. Herp., Ed. 2, Vol. 4, 1842, p. 53, pl. 14. 
Storeria dekayi BAIRD and GIRARD

Cat. N. Amer. Rept., Pt. 1, 1853, p. 135.

Storeria dekayi Cope

Ann. Rep. U. S. Nat. Mus., 1898 (1900), p. 1000, fig. 268.

TYPE LOCALity: Massachusetts and New York.

RANGe: Eastern North America, Ontario to Vera Cruz, west to

Kansas.

Storeria occipito-maculata (STORER)

RED-BELLIED SNAKE

Coluber occipito-maculatus STORER

Rep. Fish. Rept. Birds Massachusetts, 1839, p. 230.

Storeria occipito-maculatus BAIRD and GIRARD

Cat. N. Amer. Rept., Pt. 1, 1853, p. 137.

Storeria occipitomaculata CoPE

Ann. Rep. U. S. Nat. Mus., 1898 (1900), p. 1003, fig. 269.

TYPE LOCALITY: Amherst, Mass.

RANGE: From Ontario and Lake Superior southward to Vera

Cruz, westward to Kansas, Iowa and North Dakota.

\title{
Storeria victa $\mathrm{H}_{\mathrm{AY}}$
}

Storeria victa $\mathrm{HAY}$

Science, Ser. 1, Vol. 19, April 8, 1892, p. 199.

TYPE LOCALITY: Oklawaha River, some distance north of Kissimee, Florida.

RANGE: Florida.

\author{
Virginia ( $G E N U S)$ \\ Baird and Girard, Cat. N. Amer. Rept., Pt. 1, 1853, p. 127 \\ TYPE: valeriae
}

Virginia valeriae valeriae BAIRD and GIRARD

Virginia valeriae $\mathrm{BAIRD}_{\mathrm{A}}$ and GIRARD

Cat. N. Amer. Rept., Pt. 1, 1853, p. 127.

Virginia valeriae COPE

Ann. Rep. U. S. Nat. Mus., 1898 (1900), p. 1006, fig. 270.

TYPE LOCALitr: Kent County, Maryland.

RANGE: New Jersey to South Carolina, west to Ohio and Tennessee.

\section{Virginia valeriae elegans KeNNicotT}

Virginia elegans Kexwicort

Proc. Ac. Nat. Sci. Phila., 1859, p. 99. 
Virginia elegans SteJNeGer and BARBour

Check List N. Amcr. Amph. Rept., Ed. 2, 1923, p. 110.

Virginia valeriae elegans BlanchaRD

Papers, Mich. Acad. Sci. Arts Letters, 1925, p. 8.

Virginia elegans Cope

Ann. Rep. U. S. Nat. Mus., 1898 (1900), p. 1007, fig. 271.

TYPE LOCALITY: Heavily timbered regions of southern Illinois. R ANGE: Indiana, Illinois and Iowa southward to Texas.

\section{Potamophis ( $G E N U S)$}

Fitzinger, Syst. Rept., 1843, p. 25

TYPE: striatulus

\section{Potamophis striatulus (LINNÉ)}

Coluber striatulus LinNÉ

Syst. Nat., Ed. 12, Vol. 1, 1766, p. 375.

Potamophis striatulus Garman

Bull. Essex Inst., Vol. 24, 1892, p. 105.

Amphiardis inornatus StejNeger and Barbour

Check List N. Amer. Amph. Rept., Ed. 2, 1923, p. 111.

Haldea striatula Cope

Ann. Rep. U. S. Nat. Mus., 1898 (1900), p. 1009, fig. 272.

Amphiardis inornatus Cope

Ann. Rep. U. S. Nat. Mus., 1898 (1900), p. 1009.

TYPE LOCALITY: Carolina.

RANGE: Virginia and Minnesota southward to Oklahoma and the

Gulf States.

\section{Tropidoclonion ( $G E N U S$ )}

Cope, Proc. Ac. Nat. Sci. Phila., 1860, p. 76

TY PE: lineatum

\section{Tropidoclonion lineatum (HaLloweLL)}

Microps lineatus HALLowelL

Proc. Ac. Nat. Sci. Phila., 1856, p. 241.

Tropidoclonion lineatum COPE

Proc. Ac. Nat. Sci. Phila., 1860, p. 76.

Tropidoclonium lineatum CopE

Ann. Rep. U. S. Nat. Mus., 1898 (1900), p. 1011, fig. 273.

TYPE LOCALITY: Kansas.

RANGE: Ohio to Iowa and southeastern South Dakota and through

Missouri, Kansas, and Arkansas to Oklahoma and Texas. 


\section{Liodytes ( $G E N U S)$}

Cope, Proc. Amer. Philos. Soc., Vol. 22, 1885, p. 194

TYPE: alleni

\section{Liodytes alleni (GARMAN)}

IIelicops alleni GarmaN

Proc. Boston Soc. Nat. Hist., Vol. 17, 1874, p. 92.

Liodytes alleni Cope

Proc. U. S. Nat. Mus., Vol. 11, 1888, p. 393.

Liodytes allenii Cope

Ann. Rep. U. S. Nat. Mus., 1898 (1900), p. 1013, fig. 274.

TYPE L OCALITY: Hawkinsville, Florida.

RANGE: Florida and Okefinokee Swamp, Georgia.

\section{Thamnophis ( $G E N U S$ )}

Fitzinger, Syst. Rept., 1843, p. 26

TYPE: sauritus

\section{GARTER - S NAKES}

\section{Thamnophis angustirostris (KENNICOTT)}

Eutaenia angustirostris KENNICOTT

Proc. Ac. Nat. Sci. Phila., 1860, p. 332.

Thamnophis angustirostris RUTHVEN

Bull. U. S. Nat. Mus., No. 61, 1908, p. 120.

Eutaenia angustirostris COPE

Ann. Rep. U. S. Nat. Mus., 1898 (1900), p. 1047, fig. 290.

Ty Pe locality: Parras, Coahuila, Mexico.

RANGE: Southeastern Arizona and southwestern New Mexico south to Coahuila and Durango.

Thamnophis butleri (Cope)

Eutaenia butleri CoPE

Proc. U. S. Nat. Mus., Vol. 11, 1889, p. 399.

Thamnophis butleri STEJNEGer

Proc. U. S. Nat. Mus., Vol. 17, 1895, p. 593.

Eutaenia butlerii COPE

Ann. Rep. U. S. Nat. Mus., 1898 (1900), p. 1031, fig. 281.

TYPE LOCALITY: Richmond, Indiana.

RANGE: Extreme western New York and western Pennsylvania, through Ohio and Indiana, southern Michigan and Wisconsin. 
Thamnophis eques (REuss)

Coluber eques Reuss

Mus. Senckenberg. Abh., Vol. 1, 1834, p. 152, pl. 8, fig. 2.

Thamnophis eques RuthveN

Bull. U. S. Nat. Mus., No. 61, 1908, p. 158.

Eutaenia eques Cope

Ann. Rep. U. S. Nat. Mus., 1898 (1900), p. 1049, figs. 291-293.

TYPE LOCALITY: Mexico.

RANGe: Utah, Arizona, New Mexico and Texas, southward to

Guatemala.

Thamnophis marcianus (BAIRD and Girard)

Eutainia marciana BAIRD and GIRARD

Cat. N. Amer. Rept., Pt. 1, 1853, p. 36.

Thamnophis marcianus RUTHVEN

Bull. U. S. Nat. Mus., No. 61, 1908, p. 58.

Eutaenia elegans marciana Cope

Ann. Rep. U. S. Nat. Mus., 1898 (1900), p. 1044, fig. 289.

TYPE LOCALIty: "Red River, Arkansas" [Oklahoma].

RANGE: Oklahoma and Texas west to southeastern California, northeastern Mexico.

\section{Thamnophis megalops (KenNicotT)}

Eutaenia megalops KenNicotT

Proc. Ac. Nat. Sci. Phila., 1860, p. 330.

Thamnophis megalops RuthveN

Bull. U. S. Nat. Mus., No. 61, 1908, p. 44.

Eutaenia megalops Cope

Ann. Rep. U. S. Nat. Mus., 1898 (1900), p. 1025, fig. 278.

ty pe locality: Tucson and Sta. Magdalena, Arizona.

RANGE: The whole Mexican plateau region north to Texas, southwestern New Mexico and Arizona, and the Cocopah Mountains, Lower California.

\section{Thamnophis ordinoides ordinoides (BAIRD and GIRARD)}

Tropidonotus ordinoides BAIRD and GIRARD

Proc. Ac. Nat. Sci. Phila., Vol. 6, 1852, p. 176.

Thamnophis ordinoides RUthveN

Bull. U. S. Nat. Mus., No. 61, 1908, p. 147.

Eutaenia elegans ordinoides CopE

Ann. Rep. U. S. Nat. Mus., 1898 (1900), p. 1046.

TyPe Locality: Puget Sound.

RANGE: Coastal region of California, Oregon, and Washington. 
Thamnophis ordinoides atratus (KENNICOTT)

Eutainia atrata KeNNICOTT

U. S. Pacific R. R. Surv., Vol. 12, Pt. 2, 1860, p. 296.

Thamnophis ordinoides atratus Van Denburgh and Slevin

Proc. Calif. Ac. Sci., Ser. 4, Vol. 8, 1918, p. 224, pl. 9.

TYPE LOCALITY: California.

RANGE: Coast region of California from Del Norte to Santa

Barbara Counties.

\section{Thamnophis ordinoides biscutatus (COPE)}

Eutaenia biscutata Cope

Proc. Ac. Nat. Sci. Phila., 1883, p. 21.

Thamnophis ordinoides biscutatus VAN Denburgh and Slevin

Proc. Calif. Ac. Sci., Ser. 4, Vol. 8, 1918, p. 245, pl. 11.

TYPE LOCALITy: Klamath Lake, Oregon.

RANGE: Southern British Columbia, Vancouver Island, to Siskiyou and Modoc Counties, California.

\section{Thamnophis ordinoides couchii (KenNICOTT)}

\section{Eutainia couchii KenNICOTT}

U. S. Pacific R. R. Surv., Vol. 10, Pt. 4, 1859, p. 10.

Thamnophis ordinoides couchii VAN DenbUrgh and Slevin

Proc. Calif. Ac. Sci., Ser. 4, Vol. 11, 1921, p. 29.

ty PE LOCALity: Pitt River, California.

RANGE: Sacramento and San Joaquin Valley in California eastward over the Sierra Nevada into Douglas and Washoe Counties, Nevada, and Ada County, Idaho.

Thamnophis ordinoides elegans (BAIRD and GIRARD)

Eutainia elegans BAIRD and Girard Cat. N. Amer. Rept., Pt. 1, 1853, p. 34.

Thamnophis ordinoides elegans RuthVEN Bull. U. S. Nat. Mus., No. 61, 1908, p. 138.

Eutaenia elegans elegans COPE

Ann. Rep. U. S. Nat. Mus., 1898 (1900), p. 1036, fig. 284.

TyPe locality: El Dorado County, California.

RANGE: Sierra Nevada slopes of Nevada and California south to San Diego County.

\section{Thamnophis ordinoides hammondii (КеNNICOTT)}

\section{Eutaenia hammondii KeNNicotT}

Proc. Ac. Nat. Sci. Phila., 1860, p. 322.

Thamnophis ordinoides hammondii VAN Denburgh and Slevin

Proc. Calif. Ac. Sci., Ser. 4, Vol. 8, 1918, p. 256. 
Eutaenia elegans couchii (part) COPE

Ann. Rep. U. S. Nat. Mus., 1898 (1900), p. 1042, fig. 288

TYPE LOCALITY: San Diego and Fort Tejon, California.

RANGE: Southern western California and adjacent Lower California.

Thamnophis ordinoides hueyi Van Denburgh and Slevin

Thamnophis ordinoides hueyi $\mathrm{V}_{\mathrm{AN}}$ Denburgh and SLEvin

Proc. Calif. Ac. Sci., Ser. 4, Vol. 13, July 25, 1923, p. 2.

type locality: Arroyo Encantado, San Pedro Martir Mts., Lower California.

Range: The San Pedro Martir Range.

Thamnophis ordinoides vagrans (BAIRD and GIRARD)

Eutainia vagrans $B_{A I R D}$ and GIRARD

Cat. N. Amer. Rept., Pt. 1, 1853, p. 35.

Thamnophis ordinoides vagrans VAN Denburgh and Slevin

Proc. Calif. Ac. Sci., Ser. 4, Vol. 8, 1918, p. 240.

Eutaenia elegans vagrans $\mathrm{COPE}$

Ann. Rep. U. S. Nat. Mus., 1898 (1900), p. 1039, fig. 287.

TYPE LOCALITY: California.

RANGE: Eastern Washington and Oregon, Idaho, south to northern Arizona and eastern California; San Pedro Martir Mountains, Lower California.

Thamnophis radix (BAIRD and Girard)

Eutainia radix $\mathrm{B}_{\mathrm{AIRD}}$ and GiRARD

Cat. N. Amer. Rept., Pt. 1, 1853, p. 34.

Thamnophis radix JORDAN

Man. Vertebr. Anim. North. U. S., Ed. 8, 1899, p. 193.

Eutaenia radix COPE

Ann. Rep. U. S. Nat. Mus., 1898 (1900), p. 1026, fig. 279.

TYPE LOCALITY: Racine, Wisconsin.

RANGE: Illinois and the Great Plains from Kansas and Missouri

to Canadian Northwest.

Thamnophis sauritus sauritus (LiNNÉ)

RIBBON-SNAKE

Coluber saurita LinNÉ

Syst. Nat., Ed. 12, Vol. 1, 1766, p. 385.

Thamnophis sauritus sauritus BLANCHARD

Papers Mich. Acad. Sci. Arts Letters, Vol. 4, 1924, p. 18.

Eutaenia saurita COPE

Ann. Rep. U. S. Nat. Mus., 1898 (1900), p. 1020, fig. 276. 
TYPE LOCALITY: Carolina.

RANGE: Michigan, Ontario, and Maine, south to Mississippi, Alabama, and Georgia.

Thamnophis sauritus proximus ( $\left.\mathrm{S}_{A Y}\right)$

Coluber proximus $\mathrm{SAY}$

Long's Exp. Rocky Mts., Vol. 1, 1823, p. 187.

Thamnophis sauritus proximus RuTHVEN

Bull. U. S. Nat. Mus., No. 61, 1908, p. 98.

Thamnophis proximus SteJNeger and Barbour

Check List N. Amer. Amph. Rept., Ed. 2, 1923, p. 115.

Eutaenia proxima COPE

Ann. Rep. U. S. Nat. Mus., 1898 (1900), p. 1022, fig. 277.

TYPE LOCALITY: Stone quarry on west side of Missouri River,

3 miles above the mouth of Boyer's River.

RANGE: Nebraska, northern Iowa and southern Wisconsin, south

to Louisiana and Texas and through Mexico to the coastal region of Yucatan and British Honduras.

\section{Thamnophis sauritus sackenii (КеNNICOTT)}

Eutaenia sackenii KeNNICOTT

Proc. Ac. Nat. Sci. Phila., 1859, p. 98.

Thamnophis sackenii STEJNEGER and BARBour

Check List N. Amer. Amph. Rept., Ed. 1, 1917, p. 102.

Thamnophis sauritus sackeni RuTHVEN

Bull. U. S. Nat. Mus., No. 61, 1908, p. 107.

Eutaenia sackenii Cope

Ann. Rep. U. S. Nat. Mus., 1898 (1900), p. 1019, fig. 275.

TYPE LOCALITY: Florida.

RANGE: Central and northern Florida to southern Mississippi.

Thamnophis sirtalis sirtalis (LiNNé)

Coluber sirtalis LINNÉ

Syst. Nat., Ed. 10, Vol. 1, 1758, p. 222.

Thamnophis sirtalis GARMAN

Bull. Essex Inst., Vol. 24, 1892, p. 104.

Eutaenia sirtalis COPE

Ann. Rep. U. S. Nat. Mus., 1898 (1900), p. 1064, figs. 298-303.

TYPE LOCALITY: Canada.

RANGE: Eastern United States and Canada, north to about the 52nd degree of latitude, west to Minnesota, Wisconsin, and Missouri. 
Thamnophis sirtalis concinnus (HALLOWELL)

Tropidonotus concinnus Hallowell

Proc. Ac. Nat. Sci. Phila., Vol. 6, 1852, p. 182.

Thamnophis sirtalis concinnus RUTHVEN

Bull. U. S. Nat. Mus., No. 61, 1908, p. 173.

Eutaenia sirtalis concinna Cope

Ann. Rep. U. S. Nat. Mus., 1898 (1900), p. 1081, fig. 306.

TYPE LOCALITY: Oregon.

RANGE: Coast region of southwestern British Columbia, Vancouver Island, Washington and Oregon, west of the Sierra Nevada.

Thamnophis sirtalis infernalis (BLAINviLLE)

Coluber infernalis Blainville

Nouv. Ann. Mus. Hist. Nat. Paris, Vol. 4, 1835, p. 291, pl. 26, figs. 3-3a.

Thamnophis sirtalis infernalis VAN Denburgh and Slevin

Proc. Calif. Ac. Sci., Ser. 4, Vol. 8, 1918, p. 198.

TYPE LOCALITY: California.

RANGE: Southern Oregon, western Nevada and California (except desert areas).

Thamnophis sirtalis parietalis ( $\left.\mathrm{SAY}_{\mathrm{A}}\right)$

Coluber parietalis $\mathrm{SAY}_{\mathrm{A}}$

Long's Exp. Rocky Mts., Vol. 1, 1823, p. 186.

Thamnophis sirtalis var. parietalis JoRDAN

Man. Vertebr. Anim. North. U. S., Ed. 8, 1899, p. 193.

Eutaenia sirtalis parietalis COPE

Ann. Rep. U. S. Nat. Mus., 1898 (1900), p. 1077, fig. 304.

TYPE LOCALITY: Stone quarry on west side of Missouri River,

3 miles above the mouth of Boyer's River.

RANGE: Iowa and Minnesota, Missouri westward to Utah, eastern Nevada, southern Idaho, Alberta and Manitoba.

\section{Coniophanes ( $G E N U S$ )}

Cope, Proc. Ac. Nat. Sci. Phila., 1860, p. 248

TYPE: fissidens

Coniophanes imperialis (BAIRD)

Taeniophis imperialis $\mathbf{B}_{\mathrm{AIRD}}$

U. S. Mex. Bound. Surv., Vol. 2, 1859, Rept., p. 23, pl. 19, fig. 1.

Coniophanes imperialis COPE

Proc. Ac. Nat. Sci. Phila., 1861, p. 74. 


\section{Coniophanes imperialis Cope}

Ann. Rep. U. S. Nat. Mus., 1898 (1900), p. 1097, fig. 313.

TYPE LOCALITy: Brownsville, Texas.

RANGE: Southwestern Texas to Vera Cruz, Mexico.

Oxybelis ( $G E N U S)$

WAGLER, Syst. Amph., 1830, p. 183

TYPE: aeneus $=$ acuminatus

Oxybelis microphthalmus Barbour and AMaral

Oxybelis microphthalmus $\mathrm{B}_{\mathrm{ARBOUR}}$ and AMARAL

Proc. New Engl. Zoöl. Club, Vol. 9, Dec. 25, 1926, p. 80.

TYPE LOCALITY: Calabasas Canyon, Arizona.

RANGE: Arizona along the International Boundary.

Leptodeira ( $G E N U S$ )

Fitzinger, Syst. Rept., 1843, p. 27

TY PE: annulata

Leptodeira septentrionalis (KenNicotT)

Dipsas septentrionalis KenNICOTT

in BAIRD, U. S. Mex. Bound. Surv., Vol. 2, 1859, Rept., p. 16, pl. 8, fig. 1.

Leptodeira septentrionalis STEJNEGER

Proc. U. S. Nat. Mus., Vol. 14, 1891, p. 505.

Sibon septentrionale CoPE

Ann. Rep. U. S. Nat. Mus., 1898 (1900), p. 1107, fig. 316.

TYPE LOCALITY: Matamoros, Mexico, and Brownsville, Texas. RANGE: Southern Texas and northeastern Mexico.

\section{Trimorphodon ( $G E N U S)$}

Cope, Proc. Ac. Nat. Sci. Phila., 1861, p. 297 TYPE: lyrophanes

Trimorphodon lyrophanes (Cope)

Lycodon lyrophanes Cope

Proc. Ac. Nat. Sci. Phila., 1860, p. 343.

Trimorphodon lyrophanes COPE

Proc. Ac. Nat. Sci. Phila., 1861, p. 297.

Trimorphodon lyrophanes CoPE

Ann. Rep. U. S. Nat. Mus., 1898 (1900), p. 1102, fig. 314.

TyPE LOCALIty: Cape St. Lucas, Lower California. RANGE: Southern Arizona and Lower California. 
Trimorphodon vandenburghi KLAUBER

Trimorphodon vandenburghi KLAUBER

Bull. Zool. Soc. San Diego, Calif., No. 1, Junc 1, 1924, p. 17.

TYPE LOCality: Wildwood Ranch, near Ramona, San Diego

Co., Calif., altitude 1520 feet.

RANGE: Kern, Imperial, Inyo, and San Diego Counties, California.

\section{Trimorphodon vilkinsonii COPE}

Trimorphodon villkinsonii COPE

Proc. Amer. Philos. Soc., Vol. 23, 1885, p. 285.

Trimorphodon villkinsonii $\mathrm{COPE}$

Ann. Rep. U. S. Nat. Mus. 1898 (1900), p. 1105.

TYPE LOCALITY: City of Chihuahua, Mexico.

RANGE: Chihuahua Mexico and extreme western Texas.

\section{Tantilla ( $G E N U S)$}

Baird and Girard, Cat. N. Amer. Rept., Pt. 1, 1853, p. 131 TYPE: coronata

Tantilla coronata BAIRD and GIRARD

Tantilla coronata BAIRD and GIRARD

Cat. N. Amer. Rept., Pt. 1, 1853, p. 131.

Tantilla coronata CoPE

Ann. Rep. U. S. Nat. Mus., 1898 (1900), p. 1114, fig. 320.

TyPE LOCAlity: Kemper County, Mississippi.

RANGE: Southeastern United States, from Virginia southward.

Tantilla eiseni STEJNeger

Tantilla eiseni StejNeger

Proc. U. S. Nat. Mus., Vol. 18, April 16, 1896, p. 117.

TyPE LOCALity: Fresno, California.

RANGE: Southern California, and northern Lower California.

Tantilla gracilis BAIRD and Girard

Tantilla gracilis BAIRD and GIRARD

Cat. N. Amer. Rept., Pt. 1, 1853, p. 132.

Tantilla gracilis COPE

Ann. Rep. U. S. Nat. Mus., 1898 (1900), p. 1111, fig. 318.

TYPE LOCALITY: Indianola, Texas.

RANGE: Missouri to Texas. 
Tantilla nigriceps КеNNICOTT

Tantilla nigriceps KennicotT

Proc. Ac. Nat. Sci. Phila., 1860, p. 328.

Tantilla nigriceps Cope

Ann. Rep. U. S. Nat. Mus., 1898 (1900), p. 1113, fig. 319.

TyPe locality: Fort Bliss, New Mexico, and Indianola to Nueces, Texas.

RANGe: Eastern and central Arizona, New Mexico, and Texas.

Tantilla planiceps (BLAINVILlE)

Coluber planiceps Blainville

Nouv. Ann. Mus. Hist. Nat. Paris, Vol. 4, 1835, p. 294, pl. 27, figs. $3-3 b$.

Tantilla planiceps Cope

Ann. Rep. U. S. Nat. Mus., 1898 (1900), p. 1113.

TYPE LOCALITY: California.

RANGE: Cape Region of Lower California.

Tantilla wilcoxi STEJNEGER

Tantilla wilcoxi STEJNEGER

Proc. U. S. Nat. Mus., Vol. 25, Sept. 8, 1902, p. 156.

type locality: Fort Huachuca, Arizona.

RANGE: Southern Arizona.

\section{ELAPIDAE (FAMILY) \\ Micrurus ( $G E N U S$ )}

WAGLer in Spix, Serp. Brazil, 1824, p. 48

TYPE: spixii

CORAL SNAKES

Micrurus fulvius fulvius (LINNÉ)

Coluber fulvius Linné

Syst. Nat., Ed. 12, Vol. 1, 1766, p. 381.

Micrurus fulvius STEJNEGER and BARBour

Check List N. Amer. Amph. Rept., Ed. 1, 1917, p. 106.

Elaps fulvius Cope

Ann. Rep. U. S. Nat. Mus., 1898 (1900), p. 1120, figs. 321, 322.

TYPE LOCALITY: Carolina.

RANGE: North Carolina to Florida; the Gulf States and Mississippi Valley States north to Ohio and Indiana. 


\section{Micrurus fulvius barbouri ScHmidt}

Micrurus fulvius barbouri ScHMDD

Bull. Antivenin Inst. Amer., Vol. 2, No. 3, Oct. 1928, p. 64.

type locality: Royal Palm Hammock, Dade Co., Florida.

RANGE: Extreme Southern Florida.

\section{Micruroides ( $G E N U S$ )}

Sснмірт, Bull. Antivenin Inst. Amer., Vol. 2, No. 3,1928, p. 63 TYPE: euryxanthus

Micruroides euryxanthus (KenNicotT)

Elaps euryxanthus KENNicotT

Proc. Ac. Nat. Sci. Phila., 1860, p. 337.

Micruroides euryxanthus Sснмidт

Bull. Antivenin Inst. Amer., Vol. 2, No. 3, Oct. 1928, p. 63.

Micrurus euryxanthus STEJNEger and Barbour

Check List N. Amer. Amph. Rept., Ed. 2, 1923, p. 119.

Elaps euryxanthus Cope

Ann. Rep. U. S. Nat. Mus., 1898 (1900), p. 1125, fig. 324.

TYPE LOCALITY: Sonora.

RANGE: Southern New Mexico and Arizona, Northern Mexico and

Tiburon Island.

\section{HYDROPHIDAE (FAMILY)}

Pelamydrus ( $G E N U S$ )

Stejneger, Proc. U. S. Nat Mus., Vol. 38, 1910, p. 111

TYPE: platurus

Pelamydrus platurus (LiNNÉ)

Anguis platura Linné

Syst. Nat., Ed. 12, Vol. 1, 1766, p. 391.

Pelamydrus platurus StejNeger

Proc. U. S. Nat. Mus., Vol. 38, 1910, p. 111.

TYPE LOCALITY: Unknown.

RANGe: Tropical Pacific and Indian Oceans; Gulf of Panama and west coast of Central America, Guayaquil, and Gulf of California near Espiritu Santo Island. 
CHECK LIST N. AMER. AMPH. REPT.

CROTALIDAE ( $F A M I L Y)$

Agkistrodon ( $G E N U S$ )

Beauvois, Trans. Amer. Philos. Soc., Vol. 4, 1799, p. 381

TYPE: mokasen

Agkistrodon mokasen BEAUvors

COPPERHEAD

Agkishodon ${ }^{1}$ mokasen Beauvois

Trans. Amer. Philos. Soc., Vol. 4, 1799, p. 370.

Ancistrodon contortrix COPE

Ann. Rep. U. S. Nat. Mus., 1898 (1900), p. 1135, fig. 326.

type locality: America.

RANGE: Massachusetts southward to northern Florida, westward

to Illinois, Arkansas, and Texas.

Agkistrodon piscivorus (LACÉPÈDE)

COTTON-MOUTH MOCCASIN

Crotalus piscivorus LACÉPÈDE

Hist. Nat. Serp., Vol. 2, 1789, table méthod., p. 130.

Ancistrodon piscivorus Cope

Ann. Rep. U. S. Nat. Mus., 1898 (1900), p. 1133, fig. 325.

TYPE LOCALITY: Carolina.

RANGE: Dismal Swamp, Virginia, to Florida and the Gulf States, and Arkansas to Illinois.

\section{Sistrurus ( $G E N U S)$}

Garman, Mem. Mus. Comp. Zoöl., Vol. 8, No. 3, 1883, p. 110 TYPE: miliarius

Sistrurus catenatus catenatus (RAFINESQUE)

MASSASAUGA

Crotalinus catenatus RAFinesque

Amer. Month. Mag. Crit. Rev., Vol. 4, No. 1, Nov. 1818, p. 41.

Sistrurus catenatus GARMAN

Mem. Mus. Comp. Zoöl., Vol. 8, No. 3, 1883, p. 176.

Sistrurus catenatus catenatus Cope

Ann. Rep. U. S. Nat. Mus., 1898 (1900), p. 1146, fig. 329.

Ty PE LOCAlity: Prairies of the upper Missouri.

Range: Central New York and Pennsylvania, through Ohio and

Iowa to Nebraska, northward into Michigan and Ontario, southward to Kansas.

Typographical error. 
Sistrurus catenatus edwardsii (BAIRD and GIRARD)

Crotalophorus edwardsii BAIRD and GIRARD

Cat. N. Amer. Rept., Pt. 1, 1853, p. 15.

Sistrurus catenatus edwardsii STEJNEGER

Ann. Rep. U. S. Nat. Mus., 1893 (1895), p. 416.

Sistrurus catenatus edwardsii COPE

Ann. Rep. U. S. Nat. Mus., 1898 (1900), p. 1144, fig. 328.

TYPE LOCALITY: Tamaulipas, Mexico.

RANGE: Oklahoma southward through western Texas and New

Mexico to northeastern Mexico.

Sistrurus miliarius (LinNÉ)

GROUND RATTLER

Crotalus miliarius Linné

Syst. Nat., Ed. 12, Vol. 1, 1766, p. 372.

Sistrurus miliarius GARMAN

Mem. Mus. Comp. Zoöl., Vol. 8, No. 3, 1883, p. 177.

Sistrurus miliarius Cope

Ann. Rep. U. S. Nat. Mus., 1898 (1900), p. 1141, fig. 327.

TYPE LOCALITY: Carolina.

RANGE: North Carolina to Florida westward to Oklahoma and

Texas, northward in the Mississippi basin to Arkansas.

Crotalus ( $G E N U S$ )

LinNÉ, Syst. Nat., Ed. 10, Vol. 1, 1758, p. 214

TYPE: horridus

RATTLESNAKES

\section{Crotalus adamanteus BeAuvois}

DIAMOND-BACK RATTLER

Crotalus adamanteus BEAUvors

Trans. Amer. Philos. Soc., Vol. 4, 1799, p. 368.

Crotalus adamanteus adamanteus COPE

Ann. Rep. U. S. Nat. Mus., 1898 (1900), p. 1161, fig. 333.

TYPE LOCALITY: United States.

RANGE: Southern North Carolina to Florida, westward to Louisiana and Arkansas.

\section{Crotalus atrox atrox (BAIRD and GIRARD) ${ }^{1}$}

Crotalus atrox BAIRD and GIRARD

Cat. N. Amer. Rept., Pt. 1, 1853, p. 5.

1 In spite of the recent recognition of Crotalus scutulatus we remain, personally, unconvinced of its validity. 
Crotalus atrox atrox Brown

Proc. Ac. Nat. Sci. Phila., 1901, p. 103.

Crotalus adamanteus scutulatus CoPE

Ann. Rep. U. S. Nat. Mus., 1898 (1900), p. 1158, fig. 332.

Crotalus adamanteus atrox $\mathrm{COPE}$

Ann. Rep. U. S. Nat. Mus., 1898 (1900), p. 1164, fig. 334.

ty Pe locality: San Pedro, Texas.

RANGE: Texas and northern Mexico to Arizona; Colorado and southern California, also Lower California.

\section{Crotalus atrox elegans SсHмid T}

Crotalus atrox elegans ScHuidT

Bull. Amer. Mus. Nat. Hist., Vol. 46, Dec. 7, 1922, p. 699.

TYPE LOCALITy: Angel de la Guardia [sic] Island, Lower Cali-

fornia.

RANGE: Angel de la Guarda Island.

Crotalus atrox lucasensis (VAN Denburgh)

Crotalus lucasensis VAN DenbURGH

Proc. Calif. Ac. Sci., Ser. 4, Vol. 10, Aug. 6, 1920, p. 29, pl. 3.

Crotalus atrox lucasensis SснміDт

Bull. Amer. Mus. Nat. Hist., Vol. 46, Dec. 7, 1922, p. 698.

TYPE locality: Agua Caliente, Lower California.

RANGE: Southern Lower California.

Crotalus atrox tortugensis (VAN Denburgh and Slevin)

Crotalus tortugensis Van Denburgh and Slevin

Proc. Calif. Ac. Sci., Ser. 4, Vol. 11, Dec. 17, 1921, p. 398. TYPE LOCALITY: Tortuga Island, Lower California.

RANGE: Same.

Crotalus cerastes HaLLOWELL SIDEIVINDER

Crotalus cerastes HALLOWELL $_{\text {Al }}$

Proc. Ac. Nat. Sci. Phila., Vol. 7, 1854, p. 95.

Crotalus cerastes COPE

Ann. Rep. U. S. Nat. Mus., 1898 (1900), p. 1196, fig. 347.

TYPE LOCALITY: Mojave Desert.

RANGE: Northeastern Lower California, southern California, southern Nevada, Arizona, southwestern Utah.

Crotalus confluentus confluentus ( $\mathrm{SAY}_{\mathrm{X}}$ )

PRAIRIE RATTLER

Crotalus confluentus $\mathrm{SAY}$

Long's Exp. Rocky Mts., Vol. 2, 1923, p. 48. 
Crotalus confluentus confluentus CopE

Ann. Rep. U. S. Nat. Mus., 1898 (1900), p. 1170, figs. 336-338.

TYPE LOCALITY: "Valley of the Arkansa" near Bell's Springs, Colorado.

RANGE: The Great Plains, from about the 96th Meridian to the

Rocky Mountains and from southern Canada to Texas.

\section{Crotalus confluentus abyssus KLAUBer}

Crotalus confluentus abyssus KLAUBER

Trans. San Diego Soc. Nat. Hist., Vol. 6, No. 3, Feb. 28, 1930, p. 114, pl. 11, fig. 1.

TYPE LOCALity: Tanner Trail $300 \mathrm{ft}$. below south rim of Grand Canyon, Coconino County, Arizona.

RANGE: Grand Canyon of the Colorado River, Arizona.

\section{Crotalus confluentus concolor (WoodBuRY)}

YELLOW RATTLESNAKE

Crotalus concolor WoopbuRY

Bull. Univ. Utah, Vol. 20, No. 6, Dec. 1, 1929 [p. 3], figs. 1-2.

Crotalus confluentus decolor KLAUBER

Trans. San Diego Soc. Nat. Hist., Vol. 6, No. 3, 1930, p. 111.

T Y PE LOCALity: King's Ranch, Garfield Co., near base of Henry

Mountains, Utah.

RANGE: The Henry Mountains region, Wayne and Garfield

Counties, Utah.

\section{Crotalus confluentus lutosus KLAUBeR ${ }^{1}$}

Crotalus confluentus lutosus KLAUBER

Trans. San Diego Soc. Nat. Hist., Vol. 6, No. 3, 1930, p. 100, pl. 10, fig. 1.

TYPE LOCALITY: 10 miles northwest of Abraham on the road to Joy, Millard County, Utah.

RANGE: Plateau region from the Rockies to the Sierras, including

Utah west of the 111th Meridian, northern and central Nevada, northern California east of the Sierras, southeastern Oregon and southern Idaho.

\section{Crotalus confluentus oreganus (HoLBRooK)}

Crotalus oreganus HoLBrook

N. Amer. Herp., Ed. 1, Vol. 4, 1840, p. 115, pl. 29 [= 24].

Crotalus confluentus oreganus KLAUBER

1 Dr. Amaral described this race as Crotalus confluentus kellyi (Bull. Antivenin Inst., Vol. 2. No. 4, Feb. 1929, p. 91), but unfortunately his type specimen proves not to belong to this form. 
Trans. San Diego Soc. Nat. Hist., Vol. 6, No. 3, 1930, p. 129, pl. 9, fig. 2.

Crotalus confluentus lucifer COPE

Ann. Rep. U. S. Nat. Mus., 1898 (1900), p. 1176, fig. 330.

TYPE LOCALITY: Columbia River.

RANGE: British Columbia to southern California, western Idaho,

Nevada, and northwestern Lower California.

\section{Crotalus enyo (Cope)}

Caudisona enyo COPE

Proc. Ac. Nat. Sci. Phila., 1861, p. 293.

Crotalus enyo CoPE

Bull. U. S. Nat. Mus., No. 1, 1875, p. 33.

Crotalus enyo COPE

Ann. Rep. U. S. Nat. Mus., 1898 (1900), p. 1189, fig. 343.

TyPe Locality: Cape St. Lucas, Lower California.

RANGE: Extreme southern Lower California.

Crotalus exsul Garman

Crotalus exsul Garman

Mem. Mus. Comp. Zoöl., Vol. 8, No. 3, 1883, p. 114.

Crotalus ruber Cope

Ann. Rep. U. S. Nat. Mus., 1898 (1900), p. 1167, fig. 335.

TYPE LOCALITY: Cerros Island, Lower California.

RANGE: Southern California north to Los Angeles County; Lower

California; Cerros Island and islands in Gulf of California.

Crotalus horridus LinNÉ

BANDED RATTLESNAKE, BLACK RATTLESNAKE, TIMBER RATTLE-

SNAKE, CANEBRAKE RATTLESNAKE (For various varLaNtS IN COLORATION)

Crotalus horridus LinNÉ

Syst. Nat., Ed. 10, Vol. 1, 1758, p. 214.

Crotalus horridus Cope

Ann. Rep. U. S. Nat. Mus., 1898 (1900), p. 1185, fig. 324.

TyPE LOCALity: America.

RANGE: Maine to Georgia, west through Louisiana to Victoria

County, Texas; the Mississippi Valley States into Iowa.

Crotalus lepidus (KennicotT)

Caudisona lepida Kennicott

Proc. Ac. Nat. Sci. Phila., 1861, p. 206.

Crotalus lepidus Cope

Proc. Ac. Nat. Sci. Phila., 1883, p. 13. 
Crotalus lepidus Cope

Ann. Rep. U. S. Nat. Mus., 1898 (1900), p. 1191, fig. 344.

type locality: Presidio and Eagle Pass, Texas.

RANGE: Border region in Texas, New Mexico, Arizona, and adjacent Mexico.

\section{Crotalus mitchellii (COPE)}

Caudisona mitchellii $\mathrm{COPE}$

Proc. Ac. Nat. Sci. Phila., 1861, p. 293.

Crotalus mitchellii CoPE

Wheeler's Surv. W. 100 Mer., Vol. 5, 1875, p. 535.

Crotalus goldmani STEJNEGER and BARBour

Check List N. Amer. Amph. Rept., Ed. 2, 1923, p. 124.

Crotalus confluentus stephensi KLAUBER

Trans. San Diego Soc. Nat. Hist., Vol. 6, No. 3, 1930, p. 108, pl. 10, fig. 2.

Crotalus mitchellii $\mathrm{COPE}$

Ann. Rep. U. S. Nat. Mus., 1898 (1900), p. 1193, figs. 345, 346.

TYPE LOCAlity: Cape St. Lucas, Lower California.

RANGE: Arizona, Dolorado Desert to southern Lower California.

Crotalus molossus BAIRD and Girard

Crotalus molossus BAIrd and Girard

Cat. N. Amer. Rept., Pt. 1, 1853, p. 10.

Crotalus molossus CoPE

Ann. Rep. U. S. Nat. Mus., 1898 (1900), p. 1154, fig. 330.

TyPE LoCALITy: Fort Webster, New Mexico.

RANGE: Southern Texas to southern Arizona, also northern Mexico and San Esteban Island.

\section{Crotalus tigris Kennicott}

Crotalus tigris Kennicott

in BAIRD, U. S. Mex. Bound. Surv., Vol. 2, 1859, Rept., p. 14, pl. 4. Crotalus tigris Cope

Ann. Rep. U. S. Nat. Mus., 1898 (1900), p. 1181, fig. 341.

TYPE LOCALITY: Deserts of the Gila and Colorado Rivers, Arizona.

RANGE: Southern California, southern Nevada, and southern Arizona.

\section{Crotalus triseriatus (WAGLFR)}

Crotalus triseriatus WAGLER

Syst. Amp., 1830, p. 176.

Crotalus pricei STEJNEger and Barbour

Clieck List N. Amer. Amph. Rept., Ed. 2, 1923, p. 125. 
Crotalus triseriatus Amaral

Bull. Antivenin Inst. Amer., Vol. 1, No. 2, 1927, p. 48, fig. 10.

TyPE LOCALITY: Mexico.

RANGE: Southern Arizona, Chihuahua and Tamaulipas south to Jalisco and Guerrero, Mexico.

\section{Crotalus willardi MeEK}

Crotalus willardi MEeK

Field Columb. Mus. Publ. Zoöl., Vol. 7, No. 1, Jan. 1906, p. 18, pl. 3.

TYPE LOCALITY: Ramsay Canyon, 7000 feet elevation, Huachuca Mountains, Arizona.

RANGE: Santa Rita Mountain region, Arizona, and northern Mexico.

\section{SYNAPSIDA $(S U B C L A S S)$}

Osborn, Science, N. S., Vol. 17, Feb. 13, 1903, p. 276

\section{TESTUDINATA (ORDER)}

OpPEL, Ordn. Rept., 1811, p. 3

KINOSTERNIDAE (FAMILY)

Sternotherus ( $G E N U S$ )

Gray, Ann. Philos., N. S., Vol. 10, Sept. 1825, p. 211

TYPE: odoratus

STINK-POTS, MUSK-TURTLES

\section{Sternotherus carinatus (GRAY)}

Aromochelys carinata GRAY

Proc. Zool. Soc. London, 1855 (Jan. 22, 1856), p. 199.

Sternotherus carinatus Stejneger

Proc. U. S. Nat. Mus., Vol. 62, art. 6, 1923, p. 2.

Kinosternon carinatum STEJNEGER and Barbour

Check List N. Amer. Amph. Rept., Ed. 1, 1917, p. 111.

TYPE LOCALITY: Louisiana.

RANGE: Mississippi River drainage, from Vicksburg, Mississippi, south; west into southeastern Texas. 
Sternotherus minor (AGASsiz)

Goniochelys minor AGAssiz

Contr. Nat. Hist. U. S., Vol. 1, 1857, p. 424.

Sternotherus minor STEJNEGER

Proc. U. S. Nat. Mus., Vol. 62, art. 6, 1923, p. 2.

ty pe locality: Mobile, Alabama; Columbus, Georgia; New

Orleans, Louisiana.

RANGE: Northern Florida to Georgia and Alabama; upper

Tennessee.

Sternotherus odoratus (LATREILLE)

Testudo odorata LATREILLE

Hist. Nat. Rept., Vol. 1, 1802, p. 122.

Sternothaerus odoratus BELL

Zool. Journ., Vol. 2, 1825, p. 307.

Kinosternon odoratum STEJNEGER and BARBouR

Check List N. Amer. Amph. Rept., Ed. 1, 1917, p. 112.

TYPE LOCAlity: Carolina.

RANGE: Eastern and southern North America from Canada south;

west to western Missouri, Oklahoma and southern Texas.

Kinosternon ( $G E N U S$ )

Sprx, Testud. Brasil, 1824, p. 17

TYPE: longicaudatum $=$ scorpioides

STINK-POTS, MUSK-TURTLES, MUD-TURTLES

Kinosternon baurii GarmaN

Cinosternum baurii Garman

Bull. Essex. Inst., Vol. 23, 1891, p. 141

TYPE LOCAlity: Key West, Florida.

RANGE: Florida.

Kinosternon baurii palmarum STEJNEGER

Kinosternon bauri palmarum STEJNEGER

Journ. Wash. Acad. Sci., Vol. 15, No. 20, Dec. 4, 1925, p. 463.

ty Pe locality: Royal Palm State Park, Dade County, Florida. RANGE: Same.

\section{Kinosternon flavescens (Agassiz)}

Platythyra flavescens AGassiz

Contr. Nat. Hist. U. S., Vol. 1, 1857, p. 430; Vol. 2, pl. 5, figs. 12-15. Cinosternum flavescens Cope

Bull. U. S. Nat. Mus., No. 1875, p. 52. 
ty Pe locality: Texas, Arizona.

RANGE: Texas, north to Kansas, Meredosia Bay of Illinois River, Morgan County, Illinois, and Colorado, west to Arizona.

\section{Kinosternon sonoriense LE Conte}

Kinosternum sonoriense LE Conte

Proc. Ac. Nat. Sci. Phila., 1853, p. 184.

Ty PE LOCALITy: Tuscon, Arizona.

RANGE: Southwestern Texas through southern New Mexico and

Arizona into southeastern California; northern Mexico.

\section{Kinosternon steindachneri SiEBENROCK}

Cinosternum steindachneri Siebenrock

Zool. Anz., Vol. 30, Oct. 16, 1906, p. 727.

ty PE LOCAlity: Orlando, Florida.

RANGE: Central Florida.

Kinosternon subrubrum subrubrum ${ }^{1}$ (LACÉPÈDE)

Testudo subrubra LACÉPÈDE

Hist. Nat. Quadr. Ovid. Serp., Vol. 1, 1788, synops. méth. tabl. between pp. 618 and 619 (comp. p. 132).

Kinosternon subrubrum subrubrum STEJNEger and BARBour

Check List N. Amer. Amph. Rept., Ed. 1, 1917, p. 112.

ty Pe locality: Pennsylvania.

RANGE: Eastern United States exclusive of peninsular Florida, west to Indiana and southeastern Illinois and Tennessee.

\section{Kinosternon subrubrum hippocrepis (GRAY)}

Kinosternon hippocrepis Gray

Proc. Zool. Soc. London, 1855 (Jan. 22, 1856), p. 198.

Kinosternon subrubrum hippocrepis STEJNeger and BARBour

Check List N. Amer. Amph. Rept., Ed. 1, 1917, p. 112.

TyPE Locality: New Orleans, Louisiana.

RANGE: Southern Alabama to Texas, north in the Mississippi

Valley to Oklahoma and southeastern Missouri.

1 This is the species usually known as Kinosternon pensiloanicum (Gmelin) 1789. Both names ate based on the same figure in Edwards's "Glanures." 


\section{CHELYDRIDAE (FAMILY)}

\section{Macrochelys ( $G E N U S$ )}

Gray, Proc. Zool. Soc. London, 1855 (Jan. 22, 1856), p. 200

TYPE: temminckii

Macrochelys temminckii (Troost)

ALLIGATOR-SNAPPER

Chelonura temminckii Troost

in Harlan's Med. Phys. Res., 1835, p. 158, footnote.

Macrochelys temminckii GraY

Proc. Zool. Soc. London, 1855 (Jan. 22, 1856), p. 200.

TYPE LOCAlity: Tributary stream of the Mississippi above

Memphis, in West Tennessee.

RANGE: Texas east to southeastern Georgia and northern Florida, north in the Mississippi basin to northern Missouri.

\section{Chelydra ( $G E N U S)$}

Schweigger, Königsberg. Arch. Naturw. Math., Vol. 1, 1812, Pt. 3, p. 292 TYPE: serpentina

Chelydra osceola Stejneger

FLORIDA SNAPPER

Chelydra osceola STEJNEGER

Proc. Biol. Soc. Washington, Vol. 31, June 24, 1918, p. 89.

ty ye locality: Clearwater, Pinellas Co., Florida.

RANGE: Central and southern Florida.

- Chelydra serpentina (LinNé)

COMMON SNAPPER

Testudo serpentina LinNé

Syst. Nat., Ed. 10, Vol. 1, 1758, p. 199.

Chelydra serpentina SCHWEIGGer

Königsberg. Arch. Naturw. Math., Vol. 1, 1812, Pt. 3, p. 293.

TYPE LOCALITY: "Warmer regions."

RANGE: Eastern North America from southern Canada and Nova

Scotia to the Gulf of Mexico and west to the Rocky Mountains. 


\section{TESTUDINIDAE (FAMILY)}

Clemmys ( $G E N U S$ )

Ritgen, Nova Acta Ac. Leopold.-Carol., Vol. 14, Pt. 1, 1828, p. 272

TYPE: punctata $=$ guttata

Clemmys guttata (SchNeIder)

SPOTTED TURTLE

Testudo guttata SCHNEIDER

Schr. Ges. Naturf. Freunde Berlin, Vol. 10, 1792, p. 264.

Clemmys guttata STRAUCH

Mém. Ac. Sci. St. Pétersbourg, Ser. 7, Vol. 5, No. 7, 1862, p. 107.

TYPE LOCALITY: Unknown.

RANGE: Eastern North America from Canada and Maine to northern Florida; in northern part west to Wisconsin and north to southern Michigan and Ontario.

\section{Clemmys insculpta (Le ConTe)}

WOOD-TURTLE

Testudo insculpta Le Conte

Ann. Lyc. Nat. Hist. New York, Vol. 3, 1830, p. 112.

Clemmys insculpta Fitzinger

Ann. Wien Mus., Vol. 1, 1835, p. 124.

TYPE LOCALITY: Northern [United] States.

RANGE: Eastern North America from Nova Scotia to Virginia, west to Michigan, Wisconsin, and Iowa; southwestern Ontario. Not found in Indiana and Illinois.

Clemmys marmorata (BAIRD and Girard)

Emys marmorata BAIRD and GIRARD

Proc. Ac. Nat. Sci. Phila., Vol. 6, 1852, p. 177.

Clemmys marmorata Strauch

Mém. Ac. Sci. St. Pétersbourg, Ser. 7, Vol. 5, No. 7, 1862, p. 108.

TyPE LOCAlity: Puget Sound, Washington.

RANGE: Pacific slope from Vancouver Island and British Columbia to San Diego, California.

\section{Clemmys muhlenbergii (SchoEPFF)}

Testudo muhlenbergii SCHOEPFF

Hist. Testud., Pt. 6, 1801, p. 132, pl. 31.

Clemmys muhlenbergii Fitzinger

Ann. Wien Mus., Vol. 1, 1835, p. 124. 
ty pe locality: Lancaster, Pennsylvania.

RANGe: New Jersey, Pennsylvania, New York, north to Lakes

George, Ontario and Erie, Rhode Island south through Maryland and Virginia to mountains of western North Carolina.

\author{
Emys ( $G$ ENUS) \\ Duméril, Zool. Anal., 1806, p. 76 \\ TYPE: europea
}

Emys blandingii (НоLвRоок)

BLANDING'S TURTLE

Cistuda blandingii HoLBrooK

N. Amer. Herp., Ed. 1, Vol. 3, 1838, p. 35, pl. 5.

Emys blandingii Strauch

Mém. Ac. Sci. St. Pétersbourg, Ser. 7, Vol. 5, No. 7, 1862, p. 28.

TYPE LOCALITy: Fox River, Illinois.

RANGE: Northern Illinois, Indiana and Wisconsin, west and north

into western Nebraska, Minnesota, Michigan and southern

Ontario; east into New England and New Jersey; Long Island.

\title{
Terrapene ( $G E N U S$ )
}

Merrem, Tent. Syst. Amph., 1820, p. 27

TYPE: clausa $=$ carolina

BOX-TURTLES

Terrapene bauri TAYLOR

Terrapene bauri $\mathrm{TAYLOR}_{\mathrm{A}}$

Proc. U. S. Nat. Mus., Vol. 17, May 11, 1895, p. 576.

TYPE LOCALITY: Florida.

RANGE: Peninsular Florida.

Terrapene carolina (LINNÉ)

Testudo carolina Linné

Syst. Nat., Ed. 10, Vol. 1, 1758, p. 198.

Terrapene carolina BELL

Zool. Journ., Vol. 2, 1825, p. 309.

TYPE LOCALITY: Carolina.

RANGE: Eastern United States from Maine to Georgia, west

to Tennessee, western Illinois, and northwards to central Michigan. 
Terrapene major (AgAssiz)

Cistudo major Agassiz

Contr. Nat. Hist. U. S., Vol. 1, 1857, p. 445.

Terrapene major BAUR

Science, Vol. 17, April 3, 1891, p. 190.

Ty PE LOCALity: Mobile, Alabama; Florida.

RANGE: Northern Florida and southern Georgia, west into southeastern Texas.

\title{
Terrapene ornata (AGASSIz)
}

Cistudo ornata AgAssiz

Contr. Nat. Hist. U. S., Vol. 1, 1857, p. 445.

Terrapene ornata $\mathrm{B}_{\mathrm{AUR}}$

Science, Vol. 17, April 3, 1891, p. 191.

TyPE LOCALITY: The Upper Missouri and Iowa.

RANGE: Territory between the Rocky Mountains and the Mississippi and Missouri Rivers, east into Iowa, western Wisconsin, Illinois and Indiana, south to the Gulf of Mexico; southwest into New Mexico, southern Arizona and northern Mexico.

Terrapene triunguis (Agassiz)

Cistudo triunguis Agassiz

Contr. Nat. Hist. U. S., Vol. 1, 1857, p. 445.

Terrapene triunguis $\mathbf{B}_{\mathrm{AUR}}$

Amer. Natural., Vol. 27, July, 1893, p. 677.

TYPE LOCALITY: New Orleans, Louisiana.

RANGE: Coastal plain of the Gulf of Mexico, north in the Mississippi basin to Missouri, west into eastern Kansas, Oklahoma and Texas.

\author{
Malaclemys ( $G E N U S$ ) \\ GraY, Cat. Tort. Brit. Mus., 1844, p. 28 \\ TY PE: concentrica \\ DIAMOND-BACK TERRAPINS
}

Malaclemys centrata centrata (LATREILLE)

Testudo centrata LATREILLE

Hist. Nat. Rept., Vol. 1, 1802, p. 145.

Malaclemys centrata LoenNBERG

Proc. U. S. Nat. Mus., Vol. 17, Nov. 15, 1894, p. 319.

TYPE LOCALITy: Carolina.

RANGE: Coast strip from neighborhood of Cape Hatteras, south to Florida. 
Malaclemys centrata concentrica (SHAw)

Testudo concentrica SHaw

Gen. Zool., Vol. 3, 1802, p. 43, pl. 9.

Malaclemmys centrata concentrica W. P. HAY

Bull. U. S. Bur. Fish., Vol. 24, 190t, p. 16.

TYPE LOCAlity: Philadelphia markets.

RANGE: Coast strip from Buzzards Bay to North Carolina, Dela-

ware and Chesapeake Bays.

Malaclemys pileata pileata (WIED)

Emys pileata WiED

Nova Acta Ac. Leopold.-Carol., Vol. 32, 1865, p. 17, pl. 1, figs. $2,3$. Malaclemmys pileata W. P. HAY

Bull. U. S. Bur. Fish., Vol. 24, 1904, p. 17.

TyPe locality: New Orleans, Louisiana.

RANGE: Coast from mouth of Mississippi River eastwards to Florida.

Malaclemys pileata littoralis (W. P. HAY)

Malaclemmys littoralis W. P. HAY

Bull. U. S. Bur. Fish., Vol. 24, 1904, p. 18, pls. 8, 9, 12, figs. 2, 3.

Malaclemys pileata littoralis Stejneger and Barbour

Check List N. Amer. Amph. Rept., Ed. 1, 1917, p. 117.

TYPE LOCALITY: Rockport, Texas.

RANGE: Coast of Texas and outlying islands.

Malaclemys pileata macrospilota (W. P. HAY)

Malaclemmys macrospilota W. P. HAY

Bull. U. S. Bur. Fish., Vol. 24, 1904, p. 16, pls. 6, 7, 11, fig. 1.

Malaclemys pileata macrospilota Stejneger and Barbour

Check List N. Amer. Amph. Rept., Ed. 1, 1917, p. 117.

TYPE LOCALity: Charlotte Harbor, Florida.

RANGE: Western coast of Florida.

\section{Graptemys ( $G E N U S$ )}

Agassiz, Contr. Nat. Hist. U. S., Vol. 1, 1857, p. 436

TYPE: geographica

MA P-TURTLES

Graptemys geographica (Le SUEUR)

Testudo geographica LE Sueur

Journ. Ac. Nat. Sci. Phila., Vol. 1, Pt. 1, 1817, p. 86, pl. 5.

Graptemys geographica Agassiz

Contr. Nat. Hist. U. S., Vol. 1, 1857, p. 436. 
TYPE LOCALITY: Narsh on the border of Lake Erie.

RANG E: Mississippi Valley north to Iowa, northern Illinois, southern Michigan, east to Pennsylvania and New York, Canada north to Ottawa River, shores of Lake Ontario, Lake George and Lake Champlain; eastern Tennessee and southwestern Virginia, west to southwestern Missouri, eastern Oklahoma, and northeastern Texas.

\section{Graptemys pseudogeographica pseudogeographica (GRAY)}

Emys pseudogeographica GraY

Syn. Rept., 1831, p. 31 ("Le Sueur" in synonymy).

Graptemys pseudogeographica GRAY

Ann. Mag. Nat. Hist., Ser. 3, Vol. 12, Sept. 1863, p. 180.

Ty Pe Locality: Wabash River, New Harmony, Indiana.

RANGE: Mississippi Valley, east to Alabama and Ohio, north to Wisconsin and northern Iowa, west to eastern Kansas and Oklahoma.

\section{Graptemys pseudogeographis kohnii (BAUR)}

Malacoclemmys kohnii $\mathbf{B}_{\text {AUR }}$

Science, Ser. 1, Vol. 16, Nov. 7, 1890, p. 263.

Graptemys pseudogeographica lohnii StejNEGER and BARBOUR

Check List N. Amer. Amph. Rept., Ed. 1, 1917, p. 117.

TYPE LOCALITy: Bayou Lafourche, B. Teche, and St. Martinsville, Louisiana.

RANGe: Gulf strip from Pensacola to eastern Texas.

Graptemys pseudogeographica oculifera (BAUR)

Malacoclemmys oculifera BAUR Science, Ser. 1, Vol. 16, Nov. 7, 1890, p. 262.

Graptemys pseudogeographica oculifera STEJNEGER and BARBOUR

Check List N. Amer. Amph. Rept., Ed. 1, 1917, p. 118.

TYPE LOCALITy: Mandeville, Louisiana.

RANGE: Southern Louisiana, west into Texas.

Graptemys pseudogeographica versa STEJNEGER

Graptemys pseudogeographica versa STEJNEGER

Journ. Washington Acad. Sci., Vol. 15, No. 20, Dec. 1925, p. 463.

TyPe locality: Austin, Texas.

RANGE: Recorded only from type locality. 


\author{
Chrysemys ( $G E N U S)$ \\ Gray, Cat. Tort. Brit. Mus., 1844, p. 27 \\ TYPE: picta \\ PAINTED TURTLES, SOLDIER TURTLES
}

Chrysemys bellii bellii (GraY)

Emys bellii GraY

Syn. Rept., 1831, p. 31.

Chrysemys bellii bellii Ruthven

Science, Vol. 59, Apr. 11, 1924, p. 340.

Chrysemys marginata bellii STEJNEGER and BARBOUR

Check List N. Amer. Amph. Rept., Ed. 2, 1923, p. 134.

Chrysemys marginata treleasi STEJNEGER and BARBOUR

Check List N. Amer. Amph. Rept., Ed. 2, 1923, p. 134.

TYPE LOCALITY: Unknown.

RANGE: From the Rocky Mountains to the Great Lakes and the

Mississippi, northwest into Washington and British Columbia east of Cascade Mountains, southwest into New Mexico and Texas.

\title{
Chrysemys bellii marginata (AgASsiz)
}

Chrysemys marginata Agassiz

Contr. Nat. Hist. U. S., Vol. 1, 1857, p. 439; Vol. 2, pl. 1, fig. 6, pl. 5, figs. 1-4.

Chrysemys bellii marginata RuTHven

Science, Vol. 59, Apr. 11, 1924, p. 340.

Chrysemys marginata marginata STEJNEger and BARBour

Check List N. Amer. Amph. Rept., Ed. 2, 1923, p. 133.

TYPE LOCALITY: Wisconsin, Michigan, Indiana, Iowa.

RANGE: Central lowland, west to the Mississippi, north into southern Wisconsin and Michigan.

\section{Chrysemys picta picta (SchNeIDER)}

Testudo picta SCHNEIDER

Naturg. Schildkr., 1783, p. 348.

Chrysemys picta (part) GraY

Cat. Shield Rept. Brit. Mus., Pt. 1, March 8, 1856, p. 32.

TYPE LOCALITY: Unknown, said to have been England.

RANGE: Eastern North America from New Brunswick to Florida.

Chrysemys picta dorsalis (Agassiz)

Chrysemys dorsalis AgAssiz

Contr. Nat. Hist. U. S., Vol. 1, 1857, p. 440. 
Chrysemys marginata dorsalis SteJneger and BARbour

Check List N. Amer. Ampl. Rept., Ed. 2, 1923, p. 134.

TYPE LOCALITY: Mississippi and Louisiana.

RANGE: Mississippi Valley north to southern Missouri.

\section{Pseudemys ( $G E N U S$ )}

Gray, Proc. Zool. Soc. London, 1855 (Jan. 22, 1856), p. 197

TYPE: concinna

SLIDERS OR COOTERS

\section{Pseudemys alabamensis BAUR}

Pseudemys alabamensis $\mathrm{B}$ AUR

Proc. Amer. Pliilos. Soc., Vol. 31, July, 1893, p. 224.

type locality: Mobile Bay, Alabama.

RANGE: Gulf coast from extreme northwestern. Florida to Louisiana.

\section{Pseudemys concinna (Le Conte)}

Testudo concinna Le Conte

Ann. Lyc. Nat. Hist. New York, Vol. 3, 1830, p. 106.

Pseudemys concinna Gray

Cat. Shield Rept. Brit. Mus., Pt. 1, March 8, 1856, p. 34.

TyPE LOCAlity: Rivers of Georgia and Carolina above Augusta and Columbia.

RANGE: Eastern rivers from northern Florida to southern Maryland.

\section{Pseudemys elegans (WIED)}

Emys elegans WIED

Reise Nord-Amer., Vol. 1, Pt. 4, 1838, p. 213.

Pseudemys elegans Cope

Bull. U. S. Nat. Mus., No. 1, 1875, p. 53.

TYPE LOCALITY: New Harmony, Indiana.

RANGE: Southern Texas and Mississippi basin north to Iowa, northern Indiana and Ohio.

\section{Pseudemys floridana (Le ConTe)}

Testudo floridana LE Conte

Ann. Lyc. Nat. Hist. New York, Vol. 3, 1830, p. 100.

Pseudemys floridana $\mathbf{B}$ AUR

Proc. Amer. Philos. Soc., Vol. 31, 1893, p. 223.

TYPE LOCALity: St. John's River, Florida.

KANGE: Florida into southern Georgia. 
Pseudemys hieroglyphica (Ноцввоок) ${ }^{1}$

Emys hieroglyphica НоцвRоок

N. Amer. Herp., Ed. 1, Vol. 1, 1836, p. 47, pl. 2.

Pseudemys hieroglyphica H. Garman

Bull. Ill. Lab. Nat. Hist., Vol. 3, 1892, p. 231.

TyPE LOCality: Cumberland River, Tennessee.

RANGE: Rivers of southern Appalachians.

\section{Pseudemys mobilensis Holbrook ${ }^{2}$}

Emys mobilensis HoLBrook

N. Amer. Herp., Ed. 1, Vol. 2, 1838, p. 53, pl. 9.

Pseudemys mobilensis True

Bull. U. S. Nat. Mus., No. 24, 1883, p. 32.

TYPE LOCALITy: Mobile, Alabama.

RANGE: Gulf coast from Texas to northern Florida; southwestern Georgia.

\section{Pseudemys ornata nebulosa (VAN Denburgh)}

Chrysemys nebulosa $\mathrm{V}_{\text {AN }}$ Denburgh

Proc. Calif. Ac. Sci., Ser. 2, Vol. 5, 1895, p. 84, pl. 4-6.

Pseudemys ornata nebulosa STEJneger and Barbour

Check List N. Amer. Amph. Rept., Ed. 1, 1917, p. 120.

TYPE LOCALITY: Los Dolores, abreast of San José Island, Lower California.

RANGE: Southern Lower California.

Pseudemys rubriventris (Le ConTe)

Testudo rubriventris Le Conte

Ann. Lyc. Nat. Hist. New York, Vol. 3, 1830, p. 101.

Pseudemys rubriventris LoennberG

Proc. U. S. Nat. Mus., Vol. 17, Nov. 15, 1894, p. 318.

TYPE Locality: Delaware River, near Trenton, New Jersey. RANGE: Atlantic coast from extreme northeast Florida to Long Island, New York; Plymouth, Massachusetts.

\section{Pseudemys scripta (SchoepfF)}

Testudo scripta SchoepfF

Hist. Testud., Pts. 1-2, 1792, p. 16, pl. 3, figs. 4, 5.

Pseudemys scripta JoRDan

Man. Vertebr. Anim. North. U. S., Ed. 8, 1899, p. 209.

1 Specimens from North Carolina have recently been described as $P$. elonae (Brimley, Journ. Elisha Mitchell Sci. Soc., Vol, 44, No. 1, 1928, p. 66, from Guilford County, North Carolina).

2 Specimens from Louisiana have recently been described as $P$, vioscana (Brimley, Journ. Elisha Mitchell Sci. Soc. Vol. 44, No. 1, 1928, p. 66, from Lake Des Allemands, Louisiana). 
TYPE LOCALITY: Unknown.

RANGE: Northern Florida to North Carolina.

Pseudemys texana BAUR

Pseudemys texana BAUR

Proc. Amer. Philos. Soc., Vol. 31, July, 1893, p. 223.

Ty P Locality: San Antonio, Texas.

RANGE: Southwestern Missouri, western Arkansas and Texas to northeastern Mexico.

\section{Pseudemys troostii (HoLbrook)}

Emys troostii Holbrook

N. Amer. Herp., Ed. 1, Vol. 1, 1836, p. 55, pl. 4.

Pseudemys troostii Cope

Bull. U. S. Nat. Mus., No. 1, 1875, p. 53.

TYPE LOCALITy: Cumberland River, Tennessee.

RANGE: Texas, and Mississippi Valley, north to northern Missouri and southern Indiana; North Carolina.

\section{Deirochelys ( $G E N U S$ )}

Agassiz, Contr. Nat. Hist. U. S., Vol. 1, 1857, p. 441

$\mathrm{T} \mathrm{Y} \mathrm{PE}:$ reticulata $=$ reticularia

Deirochelys reticularia (LATREILLE)

CHICKEN-TURTLE

Testudo reticularia LATREILLE

Hist. Nat. Rept., Vol. 1, 1802, p. 124.

Deirochelys reticularia (part) GraY

Suppl. Cat. Shield Rept. Brit. Mus., Pt. 1, 1870, p. 39.

TYPE LOCALITY: Carolina.

RANGE: Coastal plain from Beaufort, North Carolina, to and in-

- cluding the Mississippi alluvial plain; north to Oklahoma; south to southern Florida; west to Texas.

Gopherus ( $G E N U S)$

RAFINesque, Atlantic Journ., Vol. 1, No. 2, 1832, p. 64 TYPE: polyphemus

G O PHER-TURTLES

\section{Gopherus agassizii (COOPER)}

Xerobates agassizii CoOPER

Proc. Calif. Ac. Sci., Vol. 2, 1863, p. 120. 
Gopherus agassizii STEJNEGER

N. Amer. Fauna, No. 7, May 31, 1893, p. 161.

TYPE LOCALITY: Mountains near Fort Mojave, California.

RANGE: Deserts of southwestern Arizona, and southeastern Cali-

fornia into Nevada and Utah; south into Sonora; Tiburon Island, Lower California.

\section{Gopherus berlandieri (AGASsiz)}

Xerobates berlandieri AgAssiz

Contr. Nat. Hist. U. S., Vol. 1, 1857, p. 447; Vol. 2, pl. 3, figs. 17-19. Gopherus berlandieri STEJNEGER

N. Amer. Fauna, No. 7, May 31, 1893, p. 161.

Ty PE Locality: Lower Rio Grande, Texas.

RANGE: Southwestern corner of Texas into northeastern Mexico.

Gopherus polyphemus (DAUDiN)

Testudo polyphemus DAUDIN

Hist. Nat. Rept., Vol. 2, 1803, p. 258.

Gopherus polyphemus Stejneger

N. Amer. Fauna, No. 7, May 31, 1893, p. 161.

Ty PE LOCALity: Savannah and Altamaha Rivers, Georgia.

RANGE: Coast from southern South Carolina to Florida and the

Mississippi River north into southern Arkansas.

\section{CHELONIIDAE ( $F A M I L Y)$}

Chelonia ( $G E N U S)$

Latreille, Hist. Nat. Rept., Vol. 1, 1802, p. 22

TYPE: mydas

GREEN-TURTLES

\section{Chelonia agassizii BocourT}

Chelonia agassizii BocourT

Ann. Sci. Nat., Ser. 5, Zool., Vol. 10, Pts. 1-3, 1868, p. 122.

TYPE LOCALITY: Guatemala, mouth of Nagualate in the Pacific. RANGE: Pacific Ocean, north to coasts of Lower California and southern California.

Chelonia mydas (Linné)

Testudo mydas LinNé

Syst. Nat., Ed. 10, Vol. 1, 1758, p. 197.

Chelonia mydas Schweigger

Königsberg. Arch. Naturw. Math., Vol. 1, 1812, Pt. 3, p. 412. 
TYPE LOCALITY: Ascension Island, etc.

RANGE: Atlantic Ocean; Gulf of Mexico, occasionally as far north as Massachusetts.

\section{Eretmochelys ( $G E N U S$ )}

Fitzinger, Syst. Rept., 1843, p. 30

TY PE: imbricata

SHELL-TURTLES, HAWKS-BILLS

Eretmochelys imbricata (LiNNÉ)

Testudo imbricata Linné

Syst. Nat., Ed. 12, Vol. 1, 1766, p. 350.

Eretmochelys imbricata AGassiz

Contr. Nat. Hist. U. S., Vol. 1, 1857, p. 381.

TyPe locality: American seas.

RANGE: Florida and Gulf coasts; occasionally as far north as

Massachusetts.

Eretmochelys squamosa (Girard)

Caretta squamosa Girard

U. S. Expl. Exped., Herp., 1858, p. 442, pl. 30, figs. 1-7.

Eretmochelys squamosa STEJNEGER

Bull. U. S. Nat. Mus., No. 58, 1907, p. 511.

TYPE LOCALITy: Sulu Seas and Indian Ocean.

RANGE: Pacific Ocean; coast of Lower California.

\section{Caretta ( $G E N U S)$}

Rafinesque, Specchio Sci., Palermo, Vol. 2, No. 9,

Sett. 1, 1814, p. 66

TXPE: nasuta $=$ caretta

LOGGERHEAD TURTLES

\section{Caretta caretta (LINNÉ)}

Testudo caretta LINNÉ

Syst. Nat., Ed. 10, Vol. 1, 1758, p. 197.

Caretta caretta STEJNEGER

Ann. Rep. U. S. Nat. Mus., 1902 (1904), p. 715.

TYPE LOCALITY: “About the American Islands."

RANGE: Atlantic Ocean, breeding as far north as Beaufort, North

Carolina; north occasionally to coast of Massachusetts. 
Caretta kempii (Garman)

BASTARD-TURTLE

Thalassochelys (Colpochelys) kempii Garman

Bull. Mus. Comp. Zoöl., Vol. 6, 1880, p. 123.

Caretta kempii SiEBENROCK

Zool. Jahrb. Suppl., Vol. 10, Pt. 3, 1909, p. 551.

TYPE LOCALITY: Gulf of Mexico.

RANGE: Northeastern part of Gulf of Mexico north to Cape Hat-

teras, and accidentally to the coast of Massachusetts.

Caretta olivacea (Eschscholtz)

Chelonia olivacea EschschoLtz

Zool. Atlas, Pt. 1, 1829, p. 2, pl. 3.

Caretta olivacea Rueppell

Neue Wirbelth. Abyssin., Amph., 1835, p. 7.

ty Pe locality: Manila Bay, P. I.

RANGE: Tropical Pacific straggling to the California coast.

\section{DERMOCHELIDAE (FAMILY)}

Dermochelys ( $G E N U S$ )

Blainville, Bull. Soc. Philom. Paris, 1816, p. 111 bis [= 119] TYPE: coriacea

\section{Dermochelys coriacea (LiNNÉ)}

Testudo coriacea Linné

Syst. Nat., Ed. 12, Vol. 1, 1766, p. 350.

Dermochelys coriacea Boulenger

Cat. Chel. Brit. Mus., 1889, p. 10.

TyPE Locality: Mediterranean Sea.

RANGE: Atlantic Ocean; occasionally on entire coast as far north as Nova Scotia.

\section{Dermochelys schlegelii (GARMAN)}

Sphargis coriacea var. schlegelii GARMaN

Bull. U. S. Nat. Mus., No. 25, 1884, p. 303.

Dermochclys schlegelii STEJNEGER

Bull. U. S. Nat. Mus., No. 58, 1907, p. 485.

TYPE LOCALITY: Tropical Pacific and Indian Ocean.

RANGE: Tropical Pacific Ocean; coasts of Lower California and southern California. 
TRIONYCHIDAE (FAMILY)

Amyda (GENUS)

Oken, Lehrb. Zool., Vol. 2, 1816, p. 348

TYPE: euphratica

SOFT-SHELLED TURTLES

Amyda emoryi (Agassiz)

Aspidonectes emoryi Agassiz

Contr. Nat. Hist. U. S., Vol. 1, 1857, p. 407; Vol. 2, pl. 6, figs. 4, 5. Amyda emoryi STEJNEger and Barbour

Check List N. Amer. Amph. Rept., Ed. 1, 1917, p. 124.

TyPE LOCAlity: Rio Grande River, near Brownsville, Texas.

RANGE: Rivers of Texas, north into southern Oklahoma and Arkansas.

\section{Amyda ferox (SCHNEIDER)}

Testudo ferox SchNeider

Naturg. Schildkr., 1783, p. 330.

Amyda ferox $\mathrm{OKEN}$

Lehrb. Zool., Vol. 2, 1816, p. 348.

TYPE LOCALITY: Savannah River, Georgia.

RANGE: South Carolina to Florida and Louisiana.

Amyda mutica (Le Sueur)

Trionyx muticus LE SUEUR

Mém. Mus. Hist. Nat. Paris, Vol. 15, Dec. 1827, p. 263, pl. 7. Amyda mutica Agassiz

Contr. Nat. Hist. U. S., Vol. 1, 1857, p. 399.

TYPE LOCALITY: Wabash River at New Harmony, Indiana.

RANGE: Mississippi River and tributaries; north to South Dakota and Minnesota; east to western Pennsylvania; west to Kansas, Oklahoma and Texas. Brazos and Colorado Rivers.

\section{Amyda spinifera (Le Sueur)}

Trionyx spiniferus Le Sueur

Mém. Mus. Hist. Nat. Paris, Vol. 15, 1827, p. 258, pl. 6. Amyda spinifera HuRTER

Trans. Ac. Sci. St. Louis, Vol. 20, 1911, p. 251.

TYPE LOCALITY: Wabash River at New Harmony, Indiana. RANGE: Mississippi River and tributaries, west to Colorado, north to Montana; St. Lawrence River and tributaries; east to Vermont, western New York, and Pennsylvania. 

INDEX 



\section{INDEX}

abacura, Farancia, 88 .

abacurus, Coluber, 88 .

Abastor, 88.

erythrogrammus, 88.

abyssus, Crotalus, 134.

Acris, 30.

gryllus, 30.

acutus, Crocodilus, 44.

Crocodylus, 44.

adamanteus, Crotalus, 132.

Adder, puffing, 91.

spreading, 91.

aeneus, Aneides, 20.

Plethodon, 20.

aesopus, Rana, 38.

aestivus, Coluber, 93.

Cyclophis, 93.

Opheodrys, 93.

affinis, Pituophis, 104.

agalma, Lampropeltis, 108.

Agama collaris, 49.

cornuta, 67.

coronata, 67.

douglassii, 67.

agassizii, Chelonia, 150.

Gopherus, 149.

Xerobates, 149.

Agkishodon mokasen, 131.

Agkistrodon, 131.

mokasen, 131.

piscivorus, 131.

alabamensis, Pseudemys, 147.

albilabris, Cystignathus, 36 .

Leptoaactylus, 36.

albus, Scaphiopus, 26.

alleganiensis, Cryptobranchus, 3 .

Salamandra, 3.

alleghaniensis, Cryptobranchus, 3. allegheniensis, Cryptobranchus, 3.

alleni, Helicops, 121.

Liodytes, 121.

allenii, Liodytes, 121.
Alligator, 44

missisipensis, 45 .

mississipiensis, 44.

mississippiensis, 45 .

Alligator-snapper, 140.

alterna, Lampropeltis, 105.

alternus, Ophibolus, 105.

alvarius, Bufo, 26.

amabilis, Diadophis, 88.

amaura, Lampropeltis, 109.

Amblystoma annulatum, 4.

aterrimum, 7.

cingulatum, 4 .

decorticatum, 5 .

jeffersonianum, 5 .

macrodactylum, 5 .

opacum, 6.

paroticum, 6.

punctatum, 6 .

talpoideum, 7.

texanum, 7 .

tigrinum, 7 .

Ambystoma, 4.

annulatum, 4.

cingulatum, 4 .

decorticatum, 5 .

epixanthum, 6.

gracile, 5 .

jeffersoniana, 5 .

jeffersonianum, $\boldsymbol{5}$.

mabeei, 5 .

macrodactyla, 6 .

macrodactylum, 6 .

maculatum, 6 .

microstomum, 7 .

opaca, 6.

opacum, 6.

paroticum, 5.

talpoideum, 6 .

tenebrosum, 8.

texanum, 7 .

tigrina, 7.

tigrinum, 7. 
Ambystomidae, 4.

Ameiva tesselata, 77.

American toad, 27.

amcricanus, Bufo, 27.

Crocodilus, 44.

Amoebopsis gilbertii, 73.

amoena, Carphophis, 87.

amoenus, Carplophiops, 87.

Coluber, 87.

Amphiardis, inornatus, 120.

Amphibia, 1.

Amphisbaenidae, 83.

Ampliuma, 2.

means, 2.

tridactylum, 2.

Amphiumidae, 2.

Amyda, 153.

emoryi, 153.

ferox, 153.

mutica, 153.

spinifera, 153.

Anaides ferreus, 20.

Ancistrodon contortrix, 131. piscivorus, 131.

Anderson tree-frog, 33.

andersonii, Hyla, 33.

Aneides, 20.

aeneus, 20.

farallonensis, 21 .

ferreus, 20.

flavipunctatus, 21.

iëcanus, 21.

lugubris, 21.

Anguidae, 69.

Anguis platura, 130.

ventralis, 69.

angustirostris, Eutaenia, 121.

Thamnophis, 121.

angustus, Sator, 59.

annectens, Pituophis, 102.

Anniella, 72.

nigra, 72.

pulchra, 72.

Anniellidae, 72.

annulata, Lampropeltis, 110.

Osceola, 110.

annulatum, Amblystoma, 4.

Ambystoma, 4. annulatus, Linguaelapsus, 4 .

Anolis, 47.

carolinensis, 47.

stejnegeri, 47.

Anolius carolinensis, 47.

Anota maccallii, 68 .

m'callii, 68 .

modesta, 68.

platyrhina, 69 .

anthonyi, Bascanion, 93.

Coluber, 93.

Diadophis, 90.

anthracinus, Eumeces, 80 .

Plestiodon, 80.

approximans, Holbrookia, 54 .

arenicolor, Hyla, 33.

areolata, Engystoma, 43.

Gastrophryne, 43.

Rana, 38.

Arizona, 101.

elegans, 101.

occidentalis, 102.

arizonae, Diadophis, 91.

Xantusia, 73.

arnyi, Diadophis, 90.

Aromochelys carinata, 137.

Ascaphidae, 25.

Ascaphus, 25.

truci, 25.

Aspidonectes emoryi, 153.

ater, Sauromalus, 51.

Dicamptodon, 7 .

aterrimus, Dicamptodon, 8.

atrata, Eutainia, 123.

atratus, Thamnophis, 123.

atrox, Crotalus, 132.

attenuata, Salamandrina, 8.

attenuatus, Batrachoseps, 8 .

augusti, Eleutherodactylus, 37 .

Hylodes, 37.

auriculata, Desmognathus, 22.

Salamandra, 22.

Uta, 55.

auriculatus, Desmognathus, 22 . aurigulus, Coluber, 93.

Drymobius, 93.

Zamenis, 93.

aurora, Rana, 39. 
Autodax farallonensis, 21 .

ferreus, 20.

lugubris, 21.

avivoca, Hyla, 33.

bacatus, Cnemidophorus, 74.

baileyi, Crotaphytus, 49.

bairdi, Coluber, 98.

Elaphe, 98.

bairdii, Coluber, 98.

Ball-snake, 86 .

Banded rattlesnake, 135 .

barbouri, Coluber, 94 .

Micrurus, 130.

Barissia, 72.

levicollis, 72 .

bartolomas, Cnemidophorus, 74.

Bascanion anthonyi, 93.

frenatum, 95.

Bascanium piceum, 95. semilineatum, 96 .

Bastard-turtle, 152.

Batrachoseps, 8.

at.tenuatus, 8 .

catalinae, 10 .

caudatus, 9.

leucopus, 9 .

major, 9.

pacificus, 9.

baudinii, Hyla, 34 .

Smilisca, 34.

bauri, Terrapene, 142.

baurii, Cinosternum, 138.

Kinosternon, 138.

becki, Sceloporus, 63 .

beldingi, Verticaria, 75 .

bellii, Chrysemys, 146.

Emys, 146.

bellona, Pityophis, 104 .

berlandieri, Gopherus, 150.

Xerobates, 150.

bilineatus, Spelerpes, 18 .

Bipes, 83.

biporus, 83 .

biporus, Bipes, 83 .

Euchirotes, 83.

biscutata, Eutaenia, 123.

biscutatus, Thamnophis, 123. bi-seriatus, Sceloporus, 63 .

bislineata, Eurycea, 17.

Salamandra, 17.

Black rattlesnake, 135 .

Black-snake, 94.

pilot, 99.

blainvillei, Phrynosoma, 66 .

blainvillii, Phrynosoma, 66 .

blandingii, Cistudo, 142.

Emys, 142.

Blanding's turtle, 142.

Blind eel, 2 .

Blue racer, 94.

Blue-tailed skink, 81 .

Boa contortrix, 91 .

Boidae, 85.

Boomer, mountain, 49.

boreas, Bufo, 27.

bottae, Charina, 86 .

Tortrix, 86.

Box-turtles, 142.

boylii, Lampropeltis, 107.

Ophibolus, 107.

Rana, 39.

brachyphona Pseudacris, 31.

brachyphonus, Chorophilus, 31.

Brevicipitidae, 43.

brevicorne, Phrynosoma, 67.

brevilineatus, Eumeces, 80.

brevirostre, Phrynosoma, 66 .

brevirostris, Tapaya, 66 .

brevis, Coleonyx, $\mathbf{4 7}$.

brimleyorum, Desmognathus, 21.

brooksi, Lampropeltis, 107.

browni, Heterodon, 91.

Phyllorhynchus, 97.

Bufo, 26.

alvarius, 26.

americanus, 27.

boreas, 27.

californicus, 27.

canorus, 28.

cognatus, 28.

columbiensis, 27.

compactilis, 28 .

copei, 27.

debilis, 28.

fowleri, 28 . 
Bufo halophila, 27. halophilus, 27. hemiophrys, 29. lentiginosus, 30 . marinus, 29. punctatus, 29. quercicus, 29. terrestris, 30 . valliceps, 30 . woodhousei, 30 . woodhousii, 30.

Bufonidae, 26.

Bull-snake, 104. western, 102.

Bullfrog, 40.

burnettii, Gerrhonotus, 70. butleri, Eutaenia, 121.

Thamnophis, 121.

butlerii, Eutaenia, 121.

caerulea, Verticaria, 76. caeruleus, Cnemidophorus, 76. cahuilae, Leptotyphlops, 85 .

Calamaria tenuis, 111.

Calamita cinereus, 34 .

California newt, 3 . californiae, Coluber, 105.

Lampropeltis, 105.

Ophibolus, 105.

Ophis, 105.

californicus, Bufo, 27.

callicephalus, Eumeces, 80.

calligaster, Coluber, 106.

Lampropeltis, 106.

Ophibolus, 106.

Callisaurus, 52.

crinitus, 52.

draconoides, 52.

gabbii, 53.

inusitatus, 53.

myurus, 53.

splendidus, 52.

ventralis, 52 .

campi, Syrrhoplıus, 38.

cana, Ficimia, 113.

Canebrake rattlesnake, 135.

canorus, Bufo, 28.

cantabrigensis, Rana, 40. canum, Gyalopion, 113.

Gyalopium, 113.

canus, Cnemidophorus, 78.

Caretta, 151.

caretta, 151.

kempii, 152.

olivacea, 152.

squamosa, 151.

caretta, Caretta, 151.

Testudo, 151.

carinata, Aromochelys, 137.

carinatum, Kinosternon, 137.

carinatus, Sternotherus, 137.

carmenensis, Dipso-saurus, 48.

carolina, Terrapene, 142.

Testudo, 142.

carolinense, Engystoma, 43.

carolinensis, Anolis, 47.

Anolius, 47.

Desmognathus, 22.

Gastrophryne, 43.

Hyla, 34.

Carphophiops amoenus, 87 .

vermis, 87 .

Carphophis, 87.

amoena, 87.

helenae, 87 .

vermis, 87 .

catalinae, Batrachoseps, 10.

catalinensis, Cnemidophorus, 75.

Dipso-saurus, 48.

Lampropeltis, 106.

catenatus, Crotalinus, 131.

Sistrurus, 131.

catenifer, Coluber, 102.

Pituophis, 102.

Pityophis, 102.

catesbeiana, Rana, 40.

catesbiana, Rana, 40.

Caudata, 1.

caudatus, Batrachoseps, 9.

Caudisona enyo, 135.

lepida, 135.

mitchellii, 136.

celaenops, Lampropeltis, 110.

celeripes, Cnemidophorus, 78.

Celuta helenae, 87.

vermis, 87 . 
Cemophora, 111.

coccinea, 111.

centrata, Malaclemys, 143.

Testudo, 143.

ceralbensis, Cnemidophorus, 75.

cerastes, Crotalus, 133.

cerroense, Phrynosoma, 66 .

Chain-snake, 106.

Chameleon, 47.

Charina, 86.

bottae, 86 .

Chelonia, 150.

agassizii, 150.

mydas, 150 .

olivacea, 152.

Cheloniidae, 150.

Chelonura temminckii, 140.

Chelydra, 140.

osceola, 140.

serpentina, 140 .

Chelydridae, 140.

Chicken-snake, 99.

Chicken-turtle, 149.

Chilomeniscus, 114.

cinctus, 114.

ephippicus, viii

punctatissimus, 114.

stramineus, 114.

Chionactis isozonus, 112. occipitalis, 112.

taylorii, 112.

chlorosoma, Coluber, 98.

Elaphe, 98.

Chondrotus aterrimus, 7.

cingulatus, 4 .

decorticatus, 5 .

paroticus, 5 .

texanus, 7.

Chorophilus brachyphonus, 31 .

feriarum, 32 .

nigritus, 31.

occidentalis, 32.

ocularis, 32.

ornatus, 33.

septentrionalis, 32 .

triseriatus, 32 .

Chrysemys, 146.

bellii, 146.
Chrysemys dorsalis, 146.

marginata, 146.

nebulosa, 148.

picta, 146.

treleasei, 146.

chrysoscelis, Hyla, 36.

Chuck-walla, 51 .

cinctus, Chilomeniscus, 114.

cinerea, Hyla, 34.

Salamandra, 10.

cinereus, Calamita, 34 .

Plethodon, 10.

Sphaerodactylus, 46.

cingulatum, Amblystoma, 4.

Ambystoma, 4.

cingulatus, Chondrotus, 4.

Cinosternum baurii, 138.

flavescens, 138.

steindachneri, 139.

cirrigera, Eurycea, 18.

Spelerpes, 18.

Cistudo blandingii, 142.

major, 143.

ornata, 143.

triunguis, 143.

clamata, Rana, 40.

clamitans, Rana, 40.

clarionensis, Uta, 55.

clarkii, Helocoetes, 31 .

Natrix, 114.

Pseudacris, 31.

Regina, 114.

Sceloporus, 60, 62 .

Clemmys, 141

guttata, 141.

insculpta, 141.

marmorata, 141.

muhlenbergii, 141.

clemsonae, Plethodon, 11.

Cnemidophorus, 74 .

bacatus, 74 .

bartolomas, 74 .

caeruleus, 76.

canus, 78.

catalinensis, 75 .

celeripes, 78 .

ceralbensis, 75 .

danheimae, 76 . 
Cnemidophorus dickersonae, 78 . disparilis, 78.

estebanensis, 75 .

grahamii, 75 .

gularis, 75 .

hyperythrus, 75 .

labialis, 76.

martyris, 78.

maximus, 77 .

melanostethus, $\mathbf{7 7}$.

multiscutatus, 78 .

mundus, 78.

perplexus, 77.

pictus, 76 .

punctilinealis, 78 .

rubidus, 79 .

sexlineatus, 77 .

stejnegeri, 79.

stejnegerii, $\mathbf{7 9}$.

tessellatus, $\mathbf{7 7}$.

vandenburghi, 79.

Coach whip, 94.

coccinea, Cemophora, 111.

Osceola, 110.

coccineus, Coluber, 111.

coeruleus, Gerrhonotus,' 70 .

cognatus, Bufo, 28.

Coleonyx, 47.

brevis, 47.

variegatus, 47 .

Collared lizard, 49.

collaris, Agama, 49.

Crotaphytus, 49.

Colpochelys kempii, 152.

Coluber, 93.

abacurus, 88.

aestivus, 93.

amoenus, 87.

anthonyi, 93.

aurigulus, 93.

bairdi, 98 .

bairdii, 98 .

barbouri, 94.

californiae, 105.

calligaster, 106.

catenifer, 102.

chlorosoma, 98.

coccineus, 111.
Coluber confinis, 99.

constrictor, 94.

couperi, 101.

edwardsii, 90.

elapsoides, 106.

emoryi, 99.

eques, 122.

erythrogrammus, 88 .

fasciatus, 117.

flagellum, 94.

flavi-gularis, 95.

flaviventris, 94.

frenatum, 95.

fulvius, 129.

getulus, 106.

girardi, $\mathbf{9 6 .}$

guttatus, 98.

infernalis, 126.

laetus, 99.

lateralis, 95 .

melanoleucus, 103.

mormon, 94.

obsoletus, 99.

occipito-maculatus, 119.

parietalis, 126.

piceus, 95.

planiceps, 129.

proximus, 125.

punctatus, 90.

quadrivittatus, 99.

rhombomaculatus, 109 .

rigidus, 116.

rosaceus, 100 .

rosaliae, 100 .

ruthveni, 96 .

saurita, 124.

sayi, 104.

schotti, 96.

semilineatus, 96 .

septemvittatus, 116 .

simus, 92.

sipedon, 116.

sirtalis, 125.

striatulus, 120 .

subocularis, 100 .

taeniatus, 96.

triangulum, 109.

vernalis, $\mathbf{9 2}$. 
Coluber vertebralis, 104. vulpinus, 100 .

Colubridae, 87.

columbiensis, Bufo, 27.

Common newt, 3. snapper, 140.

compactilis, Bufo, 28 .

compressicauda, Natrix, 115.

Nerodia, 115.

Compsosoma couperii, 101.

concentrica, Malaclemmys, 144.

Malaclemys, 144.

Testudo, 144.

concinna, Eutaenia, 126.

Pseudemys, 147.

Testudo, 147.

Uta, 56.

concinnus, Thamnophis, 126.

Tropidonotus, 126.

concolor, Crotalus, 134.

confinis, Coluber, 99.

Elaphe, 99.

Scotophis, 99.

confluens, Natrix, 117.

confluentus, Crotalus, 133.

Congo eel, 2 .

snake, 2.

Coniophanes, 126.

imperialis, 126.

conjuncta, Lampropeltis, 107.

consobrinus, Sceloporus, 60 .

constrictor, Coluber, 94.

Zamenis, 94.

Contia, 111.

mitis, 111.

pygaea, 118.

taylori, 112.

tenuis, 111.

contortrix, Ancistrodon, 131.

Boa, 91.

Heterodon, 91.

Cooters, 147.

copei, Bufo, 27.

Cophosaurus texanus, 55.

Copperhead, 131.

Coral-snakes, 129.

Cordylus multi-carinatus, 70.

coriacea, Dermochelys, 152. coriacea, Testudo, 152.

Corn-snake, 98.

cornuta, Agama, 67.

cornutum, Phrynosoma, 67.

coronata, Agama, 67.

Tantilla, 128.

coronatum, Phrynosoma, 67.

Costata, 25.

Cotton-mouth moccasin, 131.

couchii, Eutaenia, 124.

Eutainia, 123.

Scaphiopus, 25.

Sceloporus, 60 .

Thamnophis, 123.

couperi, Coluber, 101.

Drymarchon, 101.

couperii, Compsosoma, 101.

Cricket-frog, 30.

crinitus, Callisaurus, 52.

croceater, Ensatina, 13.

Plethodon, 13.

Crocodile, 44.

Crocodilus acutus, 44.

americanus, 44.

mississipiensis, 44.

Crocodylidae, 44.

Crocodylus, 44. acutus, 44.

Crotalidae, 131.

Crotalinus catenatus, 131.

Crotalophorus edwardsii, 132.

Crotalus, 132.

abyssus, 134.

adamanteus, 132.

atrox, 132.

cerastes, 133.

concolor, 134.

confluentus, 133.

decolor, 134.

elegans, 133.

enyo, 135.

exsul, 135.

goldmani, 136.

horridus, 135.

lepidus, 135.

lucasensis, 133.

lucifer, 135.

lutosus, 134. 
Crotalus miliarius, 132. mitchellii, 136. molossus, 136. oreganus, 134. piscivorus, 131. pricei, 136.

ruber, 135.

scutulatus, 133.

stephensi, 136.

tigris, 136.

tortugensis, 133

triseriatus, 136.

willardi, 137.

Crotaphytus, 49.

baileyi, 49 .

collaris, 49.

dickersonae, 50 .

dorsalis, 48.

insularis, 50.

reticulatus, 50 .

silus, 50 .

wislizenii, 50.

crucifer, Hyla, 34.

Cryptobranchidae, 2.

Cryptobranchus, 2.

alleganiensis, 3.

alleghaniensis, 3 .

allegheniensis, 3 .

Ctenosaura, 48.

hemilopha, 48.

multispinis, 48 .

cuneus, Hypopachus, 43.

cyanogenys, Sceloporus, 64 .

Cyclophis aestivus, 93.

cyclopion, Natrix, 115.

Tropidonotus, 115.

cyclopium, Natrix, 115.

Cyclura hemilopha, 48.

Cystignathus albilabris, 36 .

danheimae, Cnemidophorus, 76 .

danielsi, Gyrinophilus, 15 .

Spelerpes, 15.

debilis, Bufo, 28.

deckerti, Elaphe, 100.

decolor, Crotalus, 134.

decorticatum, Amblystoma, 5 .

Ambystoma, 5. decorticatus, Chondrotus, 5 .

decurtata, Phimothyra, 97.

decurtatus, Phyllorhynchus, 97.

Deirochelys, 149.

reticularia, 149.

dekayi, Storeria, 118.

Tropidonotus, 118.

DeKay's snake, 118.

deppei, Elaphis, 103.

Pituophis, 103.

Dermochelidae, 152.

Dermochely's, 152.

coriacea, 152.

schlegelii, 152.

deserticola, Pituophis, 102.

Desmognathus, 21.

auriculata, 22.

auriculatus, 22.

brimleyorum, 21.

carolinensis, 22.

fusca, 22.

fuscus, 21.

monticola, 22.

ochrophaea, 22.

ochrophaeus, 22.

phoca, 22.

quadra-maculatus, 23.

quadrimaculata, 23.

Diadophis, 88.

amabilis, 88.

anthonyi, 90.

arizonae, 91.

arnyi, 90.

edwardsii, 90.

modestus, 89.

occidentalis, 89 .

pulchellus, 89.

punctatus, 90.

regalis, 91.

similis, 89 .

stictogenys, 90 .

vandenburghi, 89.

Diamond-back rattler, 132. terrapins, 143.

Diapsida, 44.

Dicamptodon, 7. aterrimum, 7 . aterrimus, 8 . 
Dicamptodon ensatus, 7 .

dickersonae, Cnemidophorus, 78. Crotaphytus, 50.

Diemyctylus meridionalis, 3.

torosus, 3.

viridescens, 4 .

Diplodactylus unctus, 46.

Dipsas septentrionalis, 127.

Dipso-saurus, 48.

carmenensis, 48.

catalinensis, 48.

dorsalis, 48 .

lucasensis, 49 .

disparilis, Cnemidophorus, 78.

Sceloporus, 60.

dissecta, Glauconia, 85 .

ditmarsi, Phrynosoma, 67.

dorsalis, Chrysemys, 146.

Crotaphytus, 48.

Dipso-saurus, 48.

Plethodon, 11.

Triturus, 4.

douglassii, Agama, 67.

Phrynosoma, 67.

draconoides, Callisaurus, 52.

draytoni, Rana, 39.

draytonii, Rana, 39.

Dromicus flavilatus, 105.

Drymarchon, 101.

couperi, 101.

melanurus, 101.

Drymobius, 101.

aurigulus, 93.

margaritiferus, 101.

dulcis, Glauconia, 84.

Leptotyphlops, 84.

Rena, 84.

duryi, Gyrinophilus, 15 .

Dusky salamander, 10.

Dwarf salamander, 16.

Ebbet, 3.

edwardsii, Coluber, 90.

Crotalophorus, 132.

Diadophis, 90.

Sistrurus, 132.

Eel, Blind, 2.

Congo, 2 .
Eel, Mud, 24.

Eft, red, 3.

egregius, Eumeces, 80 .

Plestiodon, 80.

eiseni, Tantilla, 128.

Elaphe, 98.

bairdi, 98.

chlorosoma, 98.

confinis, 99.

deckerti, 100.

guttata, 98.

laeta, 99.

lindheimeri, viii.

obsoleta, 99.

quadrivittata, 99.

rosacea, 100.

rosaliae, 100.

subocularis, 100.

vulpina, 100.

vulpinus, 100 .

Elaphis deppei, 103.

guttatus, 98.

obsoletus, 99.

Elapidae, 129.

Elaps euryxanthus, 130. fulvius, 129.

elapsoidea, Osceola, 106.

elapsoides, Coluber, 106.

Lampropeltis, 106.

elegans, Arizona, 101.

Crotalus, 133.

Emys, 147.

Eutaenia, 123.

Eutainia, 123.

Holbrookia, 53.

Pseudemys, 147.

Rhinechis, 102.

Thamnophis, 123.

Uta, 58.

Virginia, 119.

Eleutherodactylus, 37. augusti, 37 .

latrans, 37.

ricordii, 37 .

Elgaria principis, 71.

elonae, Pseudemys, 148.

elongatus, Plethodon, 11. Sceloporus, 61. 
emoryi, Amyda, 153.

Aspidonectes, 153.

Coluber, 99.

Emys, 142.

bellii, 146 .

blandingii, 142.

elegans, 147.

hieroglyphica, 148.

marmorata, 141.

mobilensis, 148.

pileata, 144 .

pseudogeographica, 145.

troostii, 149.

Engystoma areolata, 43.

carolinense, 43.

texense, 43 .

Ensatina, 13.

croceater, 13.

eschscholtzii, 13.

sierrae, 14.

ensatus, Dicamptodon, 7.

Triton, 7.

enyo, Caudisona, 135.

Crotalus, 135.

ephippicus, Chilomeniscus, viii.

Epiglottophis, pleurostictus, 103.

epipleurotus, Eumeces, 81.

epixanthum, Ambystoma, 6.

eques, Coluber, 122.

Eutaenia, 122.

Thamnophis, 122.

Eretmochelys, 151.

imbricata, 151.

squamosa, 151.

erythrogaster, Natrix, 117.

erythrogrammus, Abastor, 88 .

Coluber, 88.

eschscholtzii, Ensatina, 13.

Plethodon, 13.

espiritensis, Verticaria, 75.

estebanensis, Cnemidophorus, 75.

Eublepharis variegatus, 47.

Euchirotes biporus, 83.

Eumeces, 80.

anthracinus, 80 .

brevilineatus, 80 .

callicephalus, 80 .

egregius, 80 .
Eumeces epipleurotus, 81.

fasciatus, 81 .

guttulatus, 82 .

humilis, 81 .

inexpcctatus, 80 .

lagunensis, 83 .

laticeps, 80 .

leptogrammus, 81.

longirostris, 81 .

multivirgatus, 81 .

obsoletus, 82 .

pachyurus, 82 .

pluvialis, 82 .

quinquelineatus, 81 .

septentrionalis, 82 .

skiltonianus, 83 .

tetragrammus, 83.

Euphryne obesus, 51.

Eurycea, 17.

bislineata, 17.

cirrigera, 18.

gutto-lineata, 18.

longicauda, 18.

lucifuga, 19.

melanopleura, 19.

montana, 16.

multiplicata, 19.

platycephala, 20.

rubra, 17.

schencki, 17.

wilderae, 18.

euryxanthus, Elaps, 130.

Micruroides, 130.

Micrurus, 130.

Eutaenia angustirostris, 121.

biscutata, 123.

butleri, 121.

butlerii, 121.

concinna, 126.

couchii, 124.

elegans, 123.

eques, 122.

hammondii, 123.

marciana, 122.

megalops, 122.

ordinoides, 122.

parietalis, 126.

proxima, 125. 
Eutaenia radix, 124.

sackenii, 125.

saurita, 124.

sirtalis, 125.

vagrans, 124.

Eutainia atrata, 123.

couchii, 123.

elegans, 123.

marciana, 122.

radix, 124.

vagrans, 124.

evittata, Hyla, 34 .

eximia, Hyla, 34.

exsul, Crotalus, 135.

extenuatum, Stilosoma, 111.

extenuatus, Stylophis, 111.

farallonensis, Aneides, 21.

Autodax, 21.

Farancia, 88.

abacura, 88 .

fasciata, Lacerta, 81 .

Natrix, 117.

fasciatus, Coluber, 117.

Eumeces, 81.

femoralis, Hyla, 35 .

Fence lizard, 65.

feriarum, Chorophilus, 32.

Helocaetes, 31.

Pseudacris, 31.

ferox, Amyda, 153.

Testudo, 153.

ferreus, Anaides, 20.

Aneides, 20.

Autodax, 20.

Ficimia, 113.

cana, 113.

streckeri, 113.

flagellum, Coluber, 94.

Zamenis, 94.

flavescens, Cinosternum, 138.

Kinosternon, 138.

Platythyra, 138.

flavi-gularis, Coluber, 95.

Psammophis, 95.

flavigularis, Masticophis, 95 .

flavilata, Rhadinaea, 105.

flavilatus, Dromicus, 105. flavilatus, Leimadophis, 105.

flavipunctatus, Aneides, 21.

Plethodon, 21.

flavissimus, Pseudotriton, 16.

flaviventris, Coluber, 94.

Florida pine lizard, southern, 65.

snapper, 140.

floridana, Lampropeltis, 107.

Pseudemys, 147.

Rhineura, 84.

Testudo, 147.

floridanum, Lepidosternon, 84 .

floridanus, Sceloporus, 64 .

Four-toed salamander, 10.

fowleri, Bufo, 28.

Fowler's toad, 28.

Fox-snake, 100.

frenatum, Bascanion, 95.

Coluber, 95.

Frog, Anderson tree, 33.

bull, 40 .

cricket, 30.

gopher, 38.

green, 40.

leopard, 41.

mink, 42.

pickerel, 41.

southern leopard, 42.

sphagnum, 42.

spring, 40.

wood, 42.

frontale, Phrynosoma, 66.

frontalis, Phrynosoma, 66.

fulvius, Coluber, 129.

Elaps, 129.

Micrurus, 129.

fulviventer, Natrix, 117.

fusca, Desmognathus, 22.

fuscus, Desmognathus, 21.

Triturus, 21.

gabbii, Callisaurus, 53.

garmani, Sceloporus, 60.

Garter-snakes, 121.

Gastrophryne, 43.

areolata, 43.

carolinensis, 43.

texensis, 43. 
Gekkonidae, 45.

gentilis, Lampropeltis, 110.

Ophibolus, 110.

Osceola, 110.

geographica, Graptemys, 144.

Testudo, 144.

Gerrhonotus, 70.

burnettii, 70 .

coeruleus, 70 .

infernalis, 70 .

kingii, 70.

multi-carinatus, 70, 71 .

nobilis, 70.

palmeri, 71 .

palmerii, 71 .

principis, 71 .

scincicauda, 71 .

webbii, 71 .

getula, Lampropeltis, 106.

getulus, Coluber, 106.

Lampropeltis, 106.

Ophibolus, 106.

Giant newt, 3.

Gila monster, 73.

gilberti, Xantusia, 73.

gilbertii, Amoebopsis, 73.

girardi, Coluber, 96.

Glass-snake, 69.

Glauconia dissecta, 85 .

dulcis, 84 .

humilis, 85 .

glutinosa, Salamandra, 11.

glutinosus, Plethodon, 11.

goldmani, Crotalus, 136.

Goniochelys minor, 138.

Gopher-frog, 38.

Gopher-snake, 101.

Gopher-turtles, 149.

Gopherus, 149.

agassizii, 149.

berlandieri, 150 .

polyphemus, 150.

gracia, Lichanura, 86.

gracile, Ambystoma, 5.

gracilipes, Hyla, 35.

gracilis, Sceloporus, 61.

Siredon, 5.

Tantilla, 128.

graciosa, Uta, 56.

graciosus, Sceloporus, 61 .

Urosaurus, 56.

grahamiae, Salvadora, 97.

grahamii, Cnemidophorus, 75.

Natrix, 115.

Regina, 115.

grandaevus, Sator, 59 .

Graptemys, 144.

geographica, 144.

kohnii, 145.

oculifera, 145.

pseudogeographica, 145.

versa, 145.

gratiosa, Hyla, 35.

Green frog, 40.

Green-snake, rough, 93. smooth, 92.

Green-turtles, 150.

Gridiron-tailed lizard, 52.

Ground rattler, 132.

grylio, Rana, 40.

gryllus, Acris, 30.

Rana, 30.

gularis, Cnemidophorus, 75 .

guttata, Clemmys, 141.

Elaphe, 98.

Testudo, 141.

guttatus, Coluber, 98.

Elaphis, 98.

gutto-lineata, Eurycea, 18.

Salamandra, 18.

guttolineatus, Spelerpes, 18.

guttulatus, Eumeces, 82.

Gyalopion canum, 113.

Gyalopium canum, 113.

Gyrinophilus, 15.

danielsi, 15.

duryi, 15 .

maculicaudus, 19 .

porphyriticus, 15.

Haldea striatula, 120.

halophila, Bufo, 27.

halophilus, Bufo, 27.

hammondii, Eutaenia, 123.

Scaphiopus, 26.

Spea, 26. 
hammondii, Thamnophis, 123.

Hawks-bills, 151.

beckscheri, Rana, 40.

heermanni, Pituophis, 102.

helenae, Carphophis, 87.

Celuta, 87.

Helicops alleni, 121.

Hell-bender, 3.

Helocaetes feriarum, 31.

Helocoetes, clarkii, 31 .

Heloderma, 73.

suspectum, 73 .

Helodermatidae, 73.

Hemidactylium, 10. pacificum, 9. scutatum, 10.

Hemidactylus, 45. turcicus, $\mathbf{4 5}$.

hemilopha, Ctenosaura, 48 . Cyclura, 48.

hemiophrys, Bufo, 28.

henshavii, Zablepsis, 73.

henshawi, Xantusia, 73.

hernandesi, Phrynosoma, 67.

Tapaya, 67.

Herpetodryas margaritiferus, 101.

herrerae, Lampropeltis, 109.

hesperis, Uta, 59.

Heterodon, 91.

browni, 91.

contortrix, 91.

nasicus, 92.

platyrhinus, 91.

simus, 92.

hexalepis, Phimothyra, 97.

Salvadora, 97.

hieroglyphica, Emys, 148.

Pseudemys, 148.

hippocrepis, Kinosternon, 139.

hispidus, Sauromalus, 51.

Hog-nose snakes, 91.

holbrooki, Lampropeltis, 107.

Holbrookia, 53.

approximans, 54 .

elegans, 53.

lacerata, 54 .

maculata, 54.

propinqua, 54 .
Holbrookia pulchra, 55.

stonei, 55.

texana, 55 .

holbrookii, Rana, 26.

Scaphiopus, 26.

Homalosaurus ventralis, $\mathbf{5 q}$.

Horn-snake, 88.

Horn-toads, 66.

horridus, Crotalus, 135.

hueyi, Thamnophis, 124.

humilis, Eumeces, 81.

Glauconia, 85.

Leptotyphlops, 85 .

Rena, 85.

Siagonodon, 85.

hurterii, Scaphiopus, 26.

Hydromantes, 20.

platycephala, 20.

platycephalus, 20.

Hydrophidae, 130.

Hyla, 33.

andersonii, 33.

arenicolor, 33.

avivoca, 33 .

baudinii, 34 .

carolinensis, 34 .

chrysoscelis, 36 .

cinerea, 34 .

crucifer, 34.

evittata, 34 .

eximia, 34 .

femoralis, 35 .

gracilipes, 35 .

gratiosa, 35.

pickeringii, 34 .

regilla, 35 .

septentrionalis, 35 .

squirella, 36 .

triseriata, 32 .

versicolor, 36 .

Hylidae, 30.

Hylodes augusti, 37.

ocularis, 32.

ricordii, 37.

hyperythra, Verticaria, 75 .

hyperythrus, Cnemidophorus, 75.

Hypopachus, 43. cuneus, 43. 
Hypsiglena, 113.

ochrorhyncha, 113.

ochrorhynchus, 113.

texana, 113.

iëcanus, Aneides, 21.

Iguanidae, 47 .

imbricata, Eretmochelys, 151.

Testudo, 151.

imperialis, Coniophanes, 126.

Taeniophis, 126.

Indigo-snake, 101.

inexpectatus, Eumeces, 80.

infernalis, Coluber, 126.

Gerrhonotus, 70.

Thamnoplis, 126.

Inornatus, Amphiardis, 120.

insculpta, Clemmys, 141.

Testudo, 141.

insularis, Crotaphytus, 50 .

intermedia, Leurognathus, 23.

Siren, 24.

intermedius, Plethodon, 11.

inusitatus, Callisaurus, 53.

isozonus, Chionactis, 112.

jamesi, Phrynosoma, 68.

jarrovii, Sceloporus, 62.

jeffersoniana, Ambystoma, 5 .

Salamandra, 5 .

jeffersonianum, Amblystoma, 5 .

Ambystoma, 5.

Joint-snake, 69.

jordani, Plethodon, 12.

kempii, Caretta, 152.

Colpochelys, 152.

Thalassochelys, 152 .

kingii, Gerrhonotus, 70.

King-snake, 106.

Kinosternidae, 137.

Kinosternon, 138.

baurii, 138.

carinatum, 137.

flavescens, 138.

hippocrepis, 139.

odoratum, 138.

palmarum, 138.
Kinosternon pensilvanicum, 139. sonoriense, 139.

steindachneri, 139.

subrubrum, 139.

kirtlandii, Natrix, 115.

Regina, 115.

kohnii, Graptemys, 145.

Malacoclemmys, 145 .

labialis, Cnemidophorus, 76 .

lacerata, Holhrookia, 54.

Lacerta, fasciata, 81.

maculata, 6.

sexlineata, 77 .

turcica, 45.

lacertina, Siren, 24.

laeta, Elaphe, 99.

laetus, Coluber, 99.

Scotophis, 99.

lagunensis, Eumeces, 83.

Lampropeltis, 105.

agalma, 108.

alterna, 105.

amaura, 109.

annulata, 110 .

boylii, 107.

brooksi, 107.

californiae, 105.

calligaster, 106.

catalinensis, 106.

celaenops, 110.

conjuncta, 107.

elapsoides, 106.

floridana, 107.

gentilis, 110 .

getula, 106.

getulus, 106.

herrerae, 109.

holbrooki, 107.

multicincta, 108.

niger, 108.

nigra, 108.

nitida, 105.

pyromelana, 109.

pyrrhomelaena, 109.

rhombomaculata, 109.

splendida, 108.

syspila, 110. 
Lampropeltis triangula, 109 triangulum, 109. virginiana, 106. yumanensis, 108. yumensis, 108.

laterale, Leiolopisma, 79. Liolepisma, 79.

lateralis, Coluber, 95. Leptophis, 95. Lysoptychus, 60. Scincus, 79.

Zamenis, 95.

laticeps, Eumeces, 80. latiremis, Rana, 40.

latrans, Eleutherodactylus, 37. Lithodytes, 37.

leberis, Natrix, 116.

lecontei, Rhinocheilus, 112. Rhinochilus, 112.

Leimadophis, flavilatus, 105 .

Leiolopisma, 79. laterale, 79.

lentiginosus, Bufo, 30 .

Leopard-frog, 41. southern, 42.

Leopard-lizard, 50. lepida, Caudisona, 135.

Lepidosternon floridanum, 84. lepidus, Crotalus, 135.

Leptodactylidae, 36.

Leptodactylus, 36 . albilabris, 36.

Leptodeira, 127. septentrionalis, 127.

leptogrammus, Eumeces, 81 .

Leptophis lateralis, 95. taeniata, 96.

Leptotyphlopidae, 84 .

Leptotyphlops, 84 . cahuilae, 85 .

dulcis, 84 .

humilis, 85 .

myopica, 85 .

slevini, 85.

leucopus, Batrachoseps, 9.

Leurognathus, 23. intermedia, 23. marmorata, 23. levicollis, Barissia, 72.

levis, Uta, 56.

lewisi, Necturus, 2 .

Lichanura, 85. gracia, 86 .

roseofusca, 85 .

trivirgata, 86.

licki, Sceloporus, 62.

lickii, Sceloporus, 62.

lindheimeri, Elaphe, viii.

lineatulus, Sceloporus, 62.

lineatum, Tropidoclonion, 120.

Tropidoclonium, 120.

lineatus, Microps, 120.

Linguaelapsus annulatus, 4.

Linguata, 25.

Liodytes, 121.

alleni, 121.

allenii, 121.

Liolepisma laterale, 79.

Liopeltis, 92.

vernalis, 92 .

Lithodytes latrans, 37. ricordii, 37 .

Litoria occidentalis, 32 .

littoralis, Malaclemmys, 144. Malaclemys, 144.

Lizard, collared, 49.

fence, 65 .

gridiron-tailed, 52 .

leopard, 50.

pine, 65 .

southern Florida pine, 65.

Lodia tenuis, 111.

lödingi, Pituophis, 103.

Loggerhead turtles, 151.

longicauda, Eurycea, 18.

Salamandra, 18.

longicaudus, Spelerpes, 19.

longirostris, Eumeces, 81.

Plestiodon, 81.

Loricata, 44.

lucasensis, Crotalus, 133.

Dipso-saurus, 49.

lucifer, Crotalus, 135.

lucifuga, Eurycea, 19.

lugubris, Aneides, 21.

Autodax, 21. 
lugubris, Salamandra, 21.

luteiventris, Rana, 41.

lutosus, Crotalus, 134.

Lycodon lyrophanes, 127.

lyrophanes, Lycodon, 127.

Trimorphodon, 127.

Lysoptychus lateralis, 60 .

mabeei, Ambystoma, 5 .

maccallii, Anota, 68.

Phrynosoma, 68.

Macrochelys, 140.

temminckii, 140.

macrodactyla, Ambystoma, 6 .

macrodactylum, Amblystoma, 6 .

Ambystoma, 6 .

macrospilota, Malaclemmys, 144.

Malaclemys, 144.

maculata, Holbrookia, 54

Lacerta, 6.

maculatum, Ambystoma, 6 .

maculicaudus, Gyrinophilus, 19.

maculo-quadrata, Salamandra, 23.

maculosa, Sirena, 1.

maculosus, Necturus, 1.

magister, Sceloporus, 62.

major, Batrachoseps, 9.

Cistudo, 143.

Terrapene, 143.

Malaclemmys concentrica, 144 .

littoralis, 144.

macrospilota, 144 .

pileata, 144 .

Malaclemys, 143.

centrata, 143.

concentrica, 144 .

littoralis, 144.

macrospilota, 144.

pileata, 144 .

Malacoclemmys kohnii, 145. oculifera, 145.

Manculus, 15.

quadridigitatus, 16.

remifer, 16.

mannophorus, Uta, 56.

Map-turtles, 144.

Marbled salamander, 6 . marciana, Eutaenia, 122. marciana, Eutainia, 122.

marcianus, Thamnophis, 122.

margaritiferus, Drymobius, 101.

Herpetodryas, 101.

marginata, Chrysemys, 146.

marginatum, Stereochilus, 14.

marginatus, Pseudotriton, 14.

Stereochilus, 14.

marina, Rana, 29.

marinus, Bufo, 29.

marmorata, Clemmys, 141.

Emys, 141.

Leurognathus, 23.

marnockii, Syrrhophus, 38.

martineusis, Lta, 56.

martyris, Cnemidophorus, 78.

Massasauga, 131.

Masticophis flavigularis, $\mathbf{9 5}$.

ruthveni, 96.

schotti, 96.

maximus, Cnemidophorus, 77.

m'callii, Anota, 68.

Phrynosoma, 68.

means, Amphiuma, 2.

Meantes, 23.

mearnsi, Uta, 56 .

mearnsii, Uta, 56.

megalops, Eutaenia, 122.

Thamnophis, 122.

melanoleucus, Coluber, 103.

Pituophis, 103.

Pityophis, 103.

melanopleura, Eurycea, 19.

melanopleurus, Spelerpes, 19.

melanostethus, Cnemidophorus, 7 .

melanurus, Drymarchon, 101.

Spilotes, 101.

Menobranchus punctatus, 2.

meridionalis, Diemyctylus, 3.

Triturus, 3.

merriami, Sceloporus, 62 .

metcalf, Plethodon, 12.

microphthalmus, Oxybelis, 127.

Microps lineatus, 120.

microscutata, Uta, 57.

microstomum, Ambystoma, 7.

microstomus, Chondrotus, 6.

Micruroides, 130. 
Micruroides euryxanthus, 130.

Micrurus, 129.

barbouri, 130.

euryxanthus, 130.

fulvius, 129.

miliarius, Crotalus, 132.

Sistrurus, 132.

Milk-snake, 109.

Mink-frog, 42.

minor, Goniochelys, 138. Sternotherus, 138.

missisipensis, Alligator, 45 .

mississipiensis, Alligator, 44.

Crocodilus, 44.

mississippiensis, Alligator, 45.

mitchellii, Caudisona, 136.

Crotalus, 136.

mitis, Contia, 111.

mobilensis, Emys, 148.

Pseudemys, 148.

Moccasin, cotton-mouth, 131. salt-water, 115.

modesta, Anota, 68.

modestum, Phrynosoma, 68.

modestus, Diadophis, 89.

mokasen, Agkishodon, 131.

Agkistrodon, 131.

Mole salamander, 6 .

snake, 109.

molossus, Crotalus, 136.

monserratensis, Sceloporus, 63.

montana, Eurycea, 16.

montanus, Pseudotriton, 16.

Spelerpes, 16.

monticola, Desmognathus, 22.

Moon-snake, 116.

mormon, Coluber, 94.

Mountain boomer, 49.

Mud-eel, 24.

Mud-snake, 88.

Mud-turtles, 138.

Mudpuppy, 1.

mugitus, Pituophis, 103.

muhlenbergii, Clemmys, 141.

Testudo, 141.

multi-carinatus, Cordylus, 70.

Gerrhonotus, 70, 71.

multicincta, Lampropeltis, 108. multicinctus, Ophibolus, 108.

multiplicata, Eurycea, 19.

multiplicatus, Spelerpes, 19.

multiscutatus, Cnemidophorus, 78.

multispinis, Ctenosaura, 48.

multivirgatum, Plestiodon, 81 .

multivirgatus, Eumeces, 81.

mundus, Cnemidophorus, 78 .

muscosa, Rana, 39.

Musk-turtles, 137, 138.

Mutabilia, 2.

mutica, Amyda, 153.

muticus, Trionyx, 153.

mydas, Chelonia, 150.

Testudo, 150.

myopica, Leptotyplops, 85 .

myopicum, Stenostoma, 85 .

myurus, Callisaurus, 53.

nasicus, Heterodon, 92.

Natrix, 114.

clarkii, 114.

compressicauda, 115 .

confluens, 117.

cyclopion, 115.

cyclopium, 115.

erythrogaster, 117.

fasciata, 117.

fulviventer, 117 .

grahamii, 115.

kirtlandii, 115.

leberis, 116.

pictiventris, 117.

rhombifera, 115 .

rigida, 116.

septemvittata, 116.

sipedon, 116.

taxispilota, 118.

transversa, 117.

valida, 118 .

nebulosa, Chrysemys, 148 .

Pseudemys, 148.

Necturidae, 1.

Necturus, 1.

lewisi, 1.

maculosus, 1.

punctatus, 2.

nelsoni, Phrynosoma, 69. 


\section{INDEX}

Neoseps, 83.

reynoldsi, 83 .

Nerodia, compressicauda, 115.

Newt, California, 3.

common, 3.

giant, 3.

niger, Lampropeltis, 108.

Ophibolus, 108.

nigra, Anniella, 72.

Lampropeltis, 108.

nigricauda, Uta, 57.

nigriceps, Tantilla, 129.

nigrita, Pseudacris, 31.

Rana, 31.

nigritus, Chorophilus, 31.

nitida, Lampropeltis, 105.

nitidus, Pseudotriton, 17.

nobilis, Gerrhonotus, 70.

nolascensis, Uta, $\mathbf{5 7}$.

notata, Uma, 53.

notatus, Sphaerodactylus, 46.

Notophthalmus torosus, 3. viridescens, 4 .

vittatus, 4 .

obesus, Euphryne, 51.

Sauromalus, 51.

obsoleta, Elaphe, 99.

obsoletum, Plestiodon, 82.

obsoletus, Coluber, 99.

Elaphis, 99.

Eumeces, 82.

occidentalis, Arizona, 102.

Chorophilus, 32.

Diadophis, 89.

Litoria, 32.

Pseudacris, 32.

Sceloporus, 63.

occipitale, Rhinostoma, 112. occipitalis, Chionactis, 112.

Sonora, 112.

occipito-maculata, Storeria, 119. occipito-maculatus, Coluber, 119. ochrophaea, Desmognathus, 22. ochrophaeus, Desmognathus, 22. ochrorhyncha, Hypsiglena, 113. ochrorhynchus, Hypsiglena, 113. ocularis, Chorophilus, 32.

ocularis, Hylodes, 32.

Pseudacris, 32.

oculifera, Graptemys, 145.

Malacoclemmys, 145.

odorata, Testudo, 138.

odoratum, Kinosternon, 138.

odoratus, Sternothaerus, 138.

Sternotherus, 138.

olivacea, Caretta, 152.

Chelonia, 152.

olympicus, Ranodon, 8.

Rhyacotriton, 8.

onca, Rana, 41.

opaca, Ambystoma, 6.

Salamandra, 6.

opacum, Amblystoma, 6.

Ambystoma, 6.

Opheodrys, 93.

aestivus, 93.

Ophibolus alternus, 105 .

boylii, 107.

californiae, 105.

calligaster, 106.

gentilis, 110 .

getulus, 106.

multicinctus, 108 .

niger, 108.

pyromelanus, 109.

pyrrhomelas, 109.

rhombomaculatus, 109 .

sayi, 107.

splendidus, 108.

syspilus, 110.

Ophis californiae, 105.

Ophisaurus, 69.

ventralis, 69 .

orcutti, Sceloporus, 64 .

orcuttii, Sceloporus, 64 .

ordinoides, Eutaenia, 122.

Thamnophis, 122.

Tropidonotus, 122.

oreganus, Crotalus, 134.

oregonensis, Plethodon, 13.

ornata, Cistudo, 143.

Pseudacris, 33.

Rana, 33.

Terrapene, 143.

Uta, 57. 
ornatissima, Tapaya, 68 . ornatissimum, Phrynosoma, 68. ornatum, Phrynosoma, 68. ornatus, Chorophilus, 33. Zamenis, 96.

Osceola annulata, 110 coccinea, 110 elapsoidea, 106. gentilis, 110. syspila, 110. triangula, 109. osceola, Chelydra, 140. Oxybelis, 127. microphthalmus, 127.

pachyurus, Eumeces, 82. Pacific tree-toad, 35. pacificum, Hemidactylium, 9. pacificus, Batrachoseps, 9.

Painted turtles, 146. palmarum, Kinosternon, 138. palmeri, Gerrhonotus, 71. Uta, 58.

palmerii, Gerrhonotus, 71 . Uta, 58.

palustris, Rana, 41 . parietalis, Coluber, 126.

Eutaenia, 126.

Thamnophis, 126. paroticum, Amblystoma, 6 . Ambystoma, 5 . paroticus, Chondrotus, 5 . parviscutata, Uta, 57 .

Patch-nose snake, 97.

Peeper, spring, 34 .

Pelamydrus, 130. platurus, 130. pensilvanicum, Kinosternon, 139. perplexus, Cnemidophorus, 77. Phimothyra decurtata, 97. hexalepis, 97.

phoca, Desmognathus, 22. Salamandra, 22.

Phrynosoma, 66.

blainvillei, 66 .

blainvillii, 66 .

brevicorne, 67 .

brevirostre, 66 .
Phrynosoma cerroense, 66.

cornutum, 67.

coronatum, 67.

ditmarsi, 67.

douglassii, 67 .

frontale, 66 .

frontalis, 66 .

hernandesi, 67 .

jamesi, 68 .

maccallii, 68 .

m'callii, 68 .

modestum, 68 .

nelsoni, 69 .

ornatissimum, 68 .

ornatum, 68.

platyrhinos, 69 .

schmidti, 69.

solare, 69.

solaris, 69 .

Phyllodactylus, 4.5.

tuberculosus, 45.

unctus, 46.

Phyllorhynchus, 97.

browni, 97.

decurtatus, 97.

piceum, Bascanion, 95.

piceus, Coluber, 95.

Zamenis, 95.

Pickerel-frog, 41 .

pickeringii, Hyla, 34 .

picta, Chrysemys, 146.

Testudo, 146.

Verticaria, 76.

pictus, Cnemidophorus, 76 . pictiventris, Natrix, 117. pileata, Emys, 144.

Malaclemmys, 144.

Malaclemys, 144.

Pilot black-snake, 99.

Pine-lizard, 65. southern Florida, 65.

Pine-snake, 103.

pipiens, Rana, 41.

piscivorus, Agkistrodon, 131.

Ancistrodon, 131.

Crotalus, 131.

Pituophis, 102. affinis, 104. 
Pituophis annectens, 102. catenifer, 102. deppei, 103.

deserticola, 102. heermanni, 102. lödingi, 103. melanoleucus, 103. mugitus, 103. ruthveni, 104. rutilus, 104 . sayi, 104. stejnegeri, 103. vertebralis, 104.

Pityophis bellona, 104 . catenifer, 102. melanoleucus, 103. sayi, 104. vertebralis, 104. planiceps, Coluber, 129. Tantilla, 129. platura, Anguis, 130. platurus, Pelamydrus, 130. platycephala, Eurycea, 20. Hydromantes, 20. platycephalus, Hydromantes, 20. Spelerpes, 20. platyrhina, Anota, 69. platyrhinos, Phrynosoma, 69. platyrhinus, Heterodon, 91. Platythyra flavescens, 138. Plestiodon anthracinus, 80. egregius, 80. longirostris, 81. multivirgatum, 81 . obsoletum, 82 . septentrionalis, 82 . skiltonianum, 83. tetragrammus, 83.

Plethodon, 10. aeneus, 20. cinereus, 10. clemsonae, 11. croceater, 13. dorsalis, 11. elongatus, 11 . eschscholtzii, 13. flavipunctatus, 21 . glutinosus, 11.

Plethodon intermedius, 11 . jordani, 12. metcalfi, 12. oregonensis, 13. shermani, 12. vandykei, 12. wehrlei, 12. welleri, 13. yonahlossee, 13. Plethodontidae, 8. pleurostictus, Epiglottophis, 103. pluvialis, Eumeces, 82. poinsettii, Sceloporus, 65. polyphemus, Gopherus, 150. Testudo, 150. porphyritica, Salamandra, 15. porphyriticus, Gyrinophilus, 15. Potamophis, 120. striatulus, 120.

Prairie rattler, 133. pretiosa, Rana, 41. pricei, Crotalus, 136. principis, Elgaria, 71. Gerrhonotus, 71. propinqua, Holbrookia, 54. Proteida, 1. proxima, Eutaenia, 125. proximus, Coluber, 125. Thamnophis, 125.

Psammophis, flavi-gularis, 95.

Pseudacris, 31. brachyphona, 31 . clarkii, 31.

feriarum, 31 . nigrita, 31 . occidentalis, 32 . ocularis, 32 . ornata, 33. septentrionalis, 32 . triseriata, 32.

Pseudemys, 147. alabamensis, 147 . concinna, 147. elegans, 147. elonae, 148. floridana, 147 . hieroglyphica, 148. mobilensis, 148. 
Pseudemys nebulosa, 148. rubriventris, 148.

scripta, 148.

texana, 149.

troostii, 149.

vioscana, 148.

Pseudobranchus, 24. striatus, 24.

pseudogeographica, Emys, 145. Graptemys, 145.

Pseudotriton, 16. flavissimus, 16. marginatus, 14 . montanus, 16. nitidus, 17. ruber, 17 . schencki, 17.

Puffing adder, 91. pulchellus, Diadophis, 89. pulchra, Holbrookia, 55.

Anniella, 72. punctatissimus, Chilomeniscus, 114. punctatum, Amblystoma, 6 . punctatus, Bufo, 29.

Coluber, 90.

Diadophis, 90.

Menobranchus, 2.

Necturus, 2. punctilinealis, Cnemidophorus, 78. pygaea, Contia, 118.

Seminatrix, 118.

pyromelana, Lampropeltis, 109. pyromelanus, Ophibolus, 109. pyrrhomelaena, Lampropeltis, 109. pyrrhomelas, Ophibolus, 109.

quadra-maculata, Salamandra, 23. quadra-maculatus, Desmognathus, 23. quadridigitata, Salamandra, 16. quadridigitatus, Manculus, 16. quadrimaculata, Desmognathus, 23. quadrivittata, Elaphe, 99. quadrivittatus, Coluber, 99.

Queen-snake, 116. quercicus, Bufo, 29. quinquelineatus, Eumeces, 81.

Racer, blue, 94. western, 94 . radix, Eutaenia, 124.

Eutainia, 124.

Thamnophis, 124.

Rain-toad, 36.

Rainbow-snake, 88.

Rana, 38.

aesopus, 38 .

areolata, 38 .

aurora, 39.

boylii, 39 .

cantabrigensis, 40 .

catesbeiana, 40 .

catesbiana, 40.

clamata, 40.

clamitans, 40.

draytoni, 39 .

draytonii, 39 .

grylio, 40.

gryllus, 30 .

heckscheri, 40.

holbrookii, 26.

latiremis, 40.

luteiventris, 41.

marina, 29.

muscosa, 39.

nigrita, 31.

onca, 41.

ornata, 33.

palustris, 41 .

pipiens, 41.

pretiosa, 41.

septentrionalis, 42.

sierrae, 39.

silvatica, 42 .

sphenocephala, 42.

sylvatica, 42 .

tarahumarae, 42.

virescens, 41.

virgatipes, 42.

Ranidae, 38.

Ranodon olympicus, 8.

rathbuni, Typhlomolge, 14.

Rattler, diamond-back, 132.

ground, 132.

prairie, 133.

Rattlesnake, banded, 135.

black, 135.

canebrake, 135 . 
Rattlesnake, timber, 135. yellow, 134.

Rattlesnakes, 132.

Red eft, 3 . salamander, 17.

Red-bellied snake, 119.

Redbacked salamander, 10 . regalis, Diadophis, 91. regilla, Hyla, 35 .

Regina clarkii, 114. grahamii, 115. kirtlandii, 115. valida, 118 .

remifer, Manculus, 16.

Rena dulcis, 84 .

humilis, 85 .

repens, Uta, 58.

Reptilia, 44.

reticularia, Deiroclielys, 149.

Testudo, 149.

reticulatus, Crotaphytus, 50 .

reynoldsi, Neoseps, 83.

Rhadinaea, 105.

flavilata, 105.

Rhinechis elegans, 102 .

Rhineura, 84. floridana, 84.

Rhinocheilus, 112. lecontei, 112.

Rhinochilus lecontei, 112.

Rhinostoma occipitale, 112. rhombifer, Tropidonotus, 115. rhombifera, Natrix, 115.

rhombomaculata, Lampropeltis, 109. rhombomaculatus, Coluber, 109. Ophibolus, 109.

Rhyacotriton, 8. olympicus, 8.

Ribbon-snake, 124.

ricordii, Eleutherodactylus, 37.

Hylodes, 37.

Lithodytes, 37.

rigida, Natrix, 116.

rigidus, Coluber, 116.

Ring-neck snakes, 88 .

riversiana, Xantusia, 74.

rosacea, Elaphe, 100.

rosaceus, Coluber, 100. rosaliae, Coluber, 100.

Elaphe, 100.

roseofusca, Lichanura, 85.

Rough green-snake, 93.

Rubber-snake, 86 .

ruber, Crotalus, 135.

Pseudotriton, 17.

Spelerpes, 17.

rubidus, Cnemidophorus, 79.

rubra, Eurycea, 17.

Salamandra, 17.

rubriventris, Pseudemys, 148.

Testudo, 148.

rufidorsum, Sceloporus, 64 .

ruthveni, Coluber, 96.

Masticophis, 96.

Pituophis, 104.

rutilus, Pituophis, 104.

sackeni, Thamnophis, 125. sackenii, Eutaenia, 125.

Thamnophis, 125.

Salamander, dusky, 10.

dwarf, 16.

four-toed, 10.

marbled, 6.

mole, 6 .

red, 17.

redbacked, 10.

slimy, 11.

spotted, 6 .

tiger, 7 .

Salamandra alleganiensis, 3 . auriculata, 22 .

bislineata, 17 .

cinerea, 10 .

glutinosa, 11.

gutto-lineata, 18.

jeffersoniana, 5 .

longicauda, 18.

lugubris, 21.

maculo-quadrata, 23.

opaca, 6.

phoca, 22.

porphyritica, 15.

quadra-maculata, 23. quadridigitata, 16.

rubra, 17. 
Salamandra scutata, 10.

symmetrica, 4.

talpoidea, 6.

texana, 7.

tigrina, 7.

Salamandridae, 3.

Salamandrina attenuata, 8.

Salientia, 25.

Salt-water moccasin, 115.

Salvadora, 97.

grahamiae, 97.

hexalepis, 97.

Sator, 59.

angustus, 59.

grandaevus, 59.

Sauria, 45.

saurita, Coluber, 124.

Eutaenia, 124.

sauritus, Thamnophis, 124 .

Sauromalus, 51.

ater, 51 .

hispidus, 51.

obesus, 51 .

slevini, 51.

townsendi, 51.

varius, 52 .

sayi, Coluber, 104.

Ophibolus, 107.

Pituophis, 104.

Pityophis, 104.

scalaris, Sceloporus, 64 .

Scaphiopodidae, 25.

Scaphiopus, 25.

albus, 26

couchii, 25.

hammondii, 26.

holbrookii, 26.

hurterii, 26.

Sceloporus, 60.

becki, 63 .

bi-seriatus, 63 .

clarkii, 60,62 .

consobrinus, 60 .

couchii, 60 .

cyanogenys, 64 .

disparilis, 60 .

elongatus, 61.

floridanus, 64 .
Sceloporus garmani, 60.

gracilis, 61.

graciosus, 61.

jarrovii, 62 .

licki, 62.

lickii, 62.

lineatulus, 62.

magister, 62 .

merriami, 62.

monserratensis, 63 .

occidentalis, 63 .

orcutti, 64 .

orcuttii, 64 .

poinsettii, 65 .

rufidorsum, 64 .

scalaris, 64 .

spinosus, 64 .

taylori, 63.

undulatus, 61,65 .

vandenburgianus, 61 .

variabilis, 65 .

woodi, 65 .

zosteromus, 65.

schencki, Eurycea, 17.

Pseudotriton, 17.

Spelerpes, 17.

schlegelii, Dermochelys, 152.

Sphargis, 152.

schmidti, Phrynosoma, 69.

Verticaria, 76.

schotti, Coluber, 96.

Masticophis, 96.

Zamenis, 96.

scincicauda, Gerrhonotus, 71.

Tropidolepis, 71.

Scincidae, 79.

Scincus lateralis, 79.

Scorpion, 81.

Scotophis confinis, 99.

laetus, 99.

vulpinus, 100.

scripta, Pseudemys, 148.

Testudo, 148.

scutata, Salamandra, 10.

scutatum, Hemidactylium, 10.

scutulatus, Crotalus, 133.

semiannulata, Sonora, 112.

semilineatum, Bascanium, 96. 
semilineatus, Coluber, 96.

Zamenis, 96.

Seminatrix, 118.

pygaea, 118.

septemvittata, Natrix, 116.

septemvittatus, Coluber, 116.

septentrionale, Sibon, 127.

septentrionalis, Chorophilus, 32.

Dipsas, 127.

Eumeces, 82.

Hyla, 35 .

Leptodeira, 127.

Plestiodon, 82.

Pseudacris, 32.

Rana, 42.

sericea, Verticaria, 76.

Serpentes, 84 .

serpentina, Chelydra, 140.

Testudo, 140.

sexlineata, Lacerta, 77.

sexlineatus, Cnemidophorus, 77.

Shell-turtles, 151.

shermani, Plethodon, 12.

Siagonodon, humilis, 85.

Sibon septentrionale, 127.

Sidewinder, 133.

sierrae, Ensatina, 14.

Rana, 39.

silus, Crotaphytus, 50.

silvatica, Rana, 42.

similis, Diadophis, 89 .

simus, Coluber, 92.

Heterodon, 92.

sipedon, Coluber, 116.

Natrix, 116.

Siredon gracilis, 5 .

Siren, 23.

intermedia, 24.

lacertina, 24.

striata, 24.

Sirena maculosa, 1.

Sirenidae, 23.

sirtalis, Coluber, 125.

Eutaenia, 125.

Thamnophis, 125.

Sistrurus, 131.

catenatus, 131.

edwardsii, 132 .
Sistrurus miliarius, 132.

skiltonianum, Plestiodon, 83.

skiltonianus, Eumeces, 83.

Skink, blue-tailed, 81.

slevini, Leptotyphlops, 85.

Sauromalus, 51.

Uta, 58.

Sliders, 147.

Slimy salamander, 11.

Smilisca baudinii, 34 .

Smooth green-snake, 92 .

Snake, ball, 86 .

black, 94.

bull, 104 .

chain, 106.

chicken, 99.

Congo, 2.

corn, 98 .

DeKay's, 118.

fox, 100 .

glass, 69 .

gopher, 101.

hog-nose, 91.

horn, 88.

indigo, 101.

joint, 69 .

king, 106.

milk, 109.

mole, 109.

moon, 116.

mud, 88 .

patch-nose, 97.

pilot black, 99 .

pine, 103.

queen, 116.

rainbow, 88 .

red-bellied, 119.

ribbon, 124.

rough green, 93.

rubber, 86 .

smooth green, 92.

water, 116.

western bull, 102 .

worm, 87.

Snakes, coral, 129.

garter, 121.

hog-nose, 84 .

ring-neck, 88 . 
Snapper, alligator, 140 .

common, 140.

Florida, 140.

Soft-shelled turtles, 153. solare, Phrynosoma, 69. solaris, Phrynosoma, 69. Soldier turtles, 146.

Sonora, 112.

occipitalis, 112.

semiannulata, 112.

taylori, 112.

sonoriense, Kinosternon, 139.

Southern Florida pine lizard, 65. leopard-frog, 42.

toad, 30.

Spadefoot, 26.

Spea hammondii, 26.

spelaeus, Typhlotriton, 14.

Spelerpes bilineatus, 18.

eirrigera, 18.

danielsi, 15.

guttolineatus, 18.

longicaudus, 19.

melanopleurus, 19.

montanus, 16.

multiplicatus, 19.

platycephalus, 20.

ruber, 17.

schencki, 17.

Sphaerodactylus, 46 .

cinereus, 46.

notatus, 46 .

Sphagnum-frog, 42.

Sphargis schlegelii, 152.

sphenocephala, Rana, 42.

Spilotes melanurus, 101.

spinifera, Amyda, 153.

spiniferus, Trionyx, 153.

spinosus, Sceloporus, 64.

splendida, Lampropeltis, 108.

splendidus, Callisaurus, 52.

Ophibolus, 108.

Spotted salamander, 6 .

turtle, 141.

Spreading adder, 91.

Spring-frog, 40.

Spring-peeper, 34 .

Squamata, 45 . squamata, Uta, 58.

squamosa, Caretta, 151.

Eretmochelys, 151.

squirella, Hyla, 36 .

stansburiana, Uta, 58 .

steindachneri, Cinosternum, 139.

Kinosternon, 139.

stejnegeri, Anolis, 47.

Cnemidophorus, 79.

Pituophis, 103.

Uta, 59.

stejnegerii, Cnemidophorus, 79.

stellata, Uta, 59.

Stellio undulatus, 65.

Stenodactylus variegatus, 47 .

Stenostoma, myopicum, 85.

stephens, Crotalus, 136.

Stereochilus, 14.

marginatum, 14.

marginatus, 14.

Sternothaerus odoratus, 138.

Sternotherus, 137.

carinatus, 137.

minor, 138.

odoratus, 138.

stictogenys, Diadophis, 90.

Stilosoma, 111.

extenuatum, 111.

Stink-pots, 137, 138.

stonei, Holbrookia, 55.

Storeria, 118.

dekayi, 118.

occipito-maculata, 119.

victa, 119.

stramineus, Chilomeniscus, 114.

streckeri, Ficimia, 113.

striata, Siren, 24.

striatula, Haldea, 120.

striatulus, Coluber, 120.

Potamophis, 120.

striatus, Pseudobranchus, 24.

Stylophis, extenuatus, 111.

subocularis, Coluber, 100.

Elaphe, 100.

subrubra, Testudo, 139.

subrubrum, Kinosternon, 139.

suspectum, Heloderma, 73.

Swifts, 74. 
sylvatica, Rana, 42.

symmetrica, Salamandra, 4.

Triturus, 4.

Uta, 57.

Synapsida, 137.

Syrrhophus, 38.

campi, 38.

marnockii, 38.

syspila, Lampropeltis, 110.

Osceola, 110.

syspilus, Ophibolus, 110.

taeniata, Leptophis, 96.

taeniatus, Coluber, 96.

Zamenis, 96.

Taeniophis imperialis, 126. talpoidea, Salamandra, 6.

talpoideum, Amblystoma, 7 .

Ambystoma, 6.

Tantilla, 128.

coronata, 128.

eiseni, 128.

gracilis, 128.

nigriceps, 129.

planiceps, 129.

wilcoxi, 129.

Tapaya brevirostris, 66 .

hernandesi, 67.

ornatissima, 68 .

tarahumarae, Rana, 42.

taxispilota, Natrix, 118.

taxispilotus, Tropidonotus, 118.

taylori, Contia, 112.

Sceloporus, 63.

Sonora, 112.

taylorii, Chionactis, 112.

Teiidae, 74.

temminckii, Chelonura, 140.

Macrochelys, 140.

tenebrosum, Ambystoma, 8.

tenuis, Calamaria, 111.

Contia, 111.

Lodia, 111.

Terrapene, 142 .

bauri, 142.

carolina, 142.

major, 143.

ornata, 143.
Terrapene triunguis, 143.

Terrapins, diamond-back, 143.

terrestris, Bufo, 30.

tesselata, Ameiva, 77.

tessellatus, Cnemidophorus, 77.

Testudinata, 137.

Testudinidae, 141.

Testudo caretta, 151.

carolina, 142.

centrata, 143.

concentrica, 144.

concinna, 147.

coriacea, 152.

ferox, 153.

floridana, 147.

geographica, 144.

guttata, 141.

imbricata, 151.

insculpta, 141.

muhlenbergii, 141.

mydas, 150.

odorata, 138.

picta, 146.

polyphemus, 150.

reticularia, 149.

rubriventris, 148 .

scripta, 148.

serpentina, 140.

subrubra, 139.

tetragrammus, Eumeces, 83.

Plestiodon, 83.

texana, Holbrookia, 55 .

Hypsiglena, 113.

Pseudemys, 149.

Salamandra, 7.

texanum, Amblystoma, 7.

Ambystoma, 7.

texanus, Chondrotus, 7 .

Cophosaurus, 55.

texense, Engystoma, 43.

texensis, Gastrophryne, 43.

thalassina, Uta, 59.

Thalassochelys kempii, 152 .

Thamnophis, 121.

angustirostris, 121 .

atratus, 123.

biscutatus, 123.

butleri, 121. 
Thamnophis concinnus, 126 .

couchii, 123.

elegans, 123.

eques, 122.

hammondii, 123.

hueyi, 124.

infernalis, 126.

marcianus, 122.

megalops, 122.

ordinoides, 122.

parietalis, 126.

proximus, 125.

radix, 124.

sackeni, 125.

sackenii, 125.

sauritus, 124.

sirtalis, 125.

vagrans, 124.

Tiger salamander, 7 .

tigrina, Ambystoma, 7.

Salamandra, 7 .

tigrinum, Amblystoma, 7 .

Ambystoma, 7.

tigris, Crotalus, 136.

Timber rattlesnake, 135.

Toad, American, 27.

Fowler's 28.

Pacific tree, 35.

rain, 36.

southern, 30 .

tree, 36.

Toads, horn, 66.

torosus, Diemyctylus, 3.

Notophthalmus, 3.

Triturus, 3.

Tortrix bottae, 86 .

tortugensis, Crotalus, 133.

townsendi, Sauromalus, 51.

transversa, Natrix, 117.

transversus, Tropidonotus, 117.

Tree-frog, Anderson, 33.

Tree-toad, 36.

Pacific, 35.

treleasei, Chrysemys, 146.

triangula, Lampropeltis, 109.

Osceola, 109.

triangulum, Coluber, 109.

Lampropeltis, 109. tridactylum, Amphiuma, 2.

Trimorphodon, 127. lyrophanes, 127. vandenburghi, 128. vilkinsonii, 128.

Trionychidae, 153.

Trionyx muticus, 153. spiniferus, 153.

triseriata, Hyla, 32. Pseudacris, 32.

triseriatus, Chorophilus, 32. Crotalus, 136.

Triton ensatus, 7.

Triturus, 3.

dorsalis, 4 .

fuscus, 21.

meridionalis, 3 .

symmetrica, 4 .

torosus, 3 .

viridescens, 3.

triunguis, Cistudo, 143.

Terrapene, 143.

trivirgata, Lichanura, 86.

troostii, Emys, 149.

Pseudemys, 149.

Tropidoclonion, 120.

lineatum, 120.

Tropidoclonium lineatum, 120.

Tropidolepis scincicauda, 71 .

Tropidonotus concinnus, 126.

cyclopion, 115.

dekayi, 118.

ordinoides, 122.

rhombifer, 115.

taxispilotus, 118.

transversus, 117.

truei, Ascaphus, 25.

tuberculosus, Phyllodactylus, 45.

turcica, Lacerta, 45.

turcicus, Hemidactylus, 45.

Turtle, bastard, 152.

Blanding's, 142.

chicken, 149.

spotted, 141.

wood, 141.

Turtles, box, 142. gopher, 149. green, 150. 
Turtles, loggerhead, 151.

map, 144.

mud, 138.

musk, 137, 138.

painted, 146.

shell, 151 .

soft-shelled, 153.

soldier, 146.

Typhlomolge, 14. rathbuni, 14 .

Typhlotriton, 14. spelaeus, 14.

Uma, 53.

notata, 53 .

unetus, Diplodactylus, 46.

Phyllodactylus, 46.

undulatus, Sceloporus, 61, 65 .

Stellio, 65.

Urosaurus graciosus, 56 .

Uta, 55.

auriculata, 55 .

clarionensis, 55.

concinna, 56 .

elegans, 58.

graciosa, 56 .

hesperis, 59.

levis, 56 .

mannophorus, 56.

martinensis, 56.

mearnsi, 56 .

mearnsii, 56 .

microscutata, 57 .

nigricauda, 57.

nolascensis, 57 .

ornata, 57.

palmeri, 58.

palmerii, 58.

parviscutata, 57 .

repens, 58 .

slevini, 58 .

squamata, 58.

stansburiana, 58 .

stejnegeri, 59.

stellata, 59 .

symmetrica, 57 .

thalassina, 59 .

wrighti, 56.

vagrans, Eutaenia, 124.

Eutainia, 124.

Thamnophis, 124.

valeriae, Virginia, 119.

valida, Natrix, 118.

Regina, 118.

valliceps, Bufo, 30 .

vandenburghi, Cnemidophorus, 79.

Diadophis, 89.

Trimorphodon, 128.

vandenburgianus, Sceloporus, 61 .

vandykei, Plethodon, 12.

variabilis, Sceloporus, 65.

variegatus, Coleonyx, 47.

Eublepharis, 47.

Stenodactylus, 47.

varius, Sauromalus, 52.

ventralis, Anguis, 69.

Callisaurus, 52.

Homalosaurus, 52.

Ophisaurus, 69.

vermis, Carphophiops, 87.

Carphophis, 87.

Celuta, 87.

vernalis, Coluber, 92.

Liopeltis, 92.

versa, Graptemys, 145.

versicolor, Hyla, 36.

vertebralis, Coluber, 104.

Pituophis, 104.

Pityophis, 104.

Verticaria beldingi, 75 .

caerulea, 76 .

espiritensis, 75 .

hyperythra, 75 .

picta, 76.

schmidti, 76 .

sericea, 76 .

victa, Storeria, 119.

vigilis, Xantusia, 74.

vilkinsonii, Trimorphodon, 128.

vioscana, Pseudemys, 148.

virescens, Rana, 41.

virgatipes, Rana, 42.

Virginia, 119.

elegans, 119.

valeriae, 119.

virginiana, Lampropeltis, 106. 
viridescens, Diemyctylus, 4. Notophthalmus, 4.

Triturus, 3.

vittatus, Notophthalmus, 4 .

vulpina, Elaphe, 100.

vulpinus, Coluber, 100.

Elaphe, 100.

Scotophis, 100.

Water-pilot, 118.

Water-snake, 116.

Waterdog, 1.

webbii, Gerrhonotus, 71 .

wehrlei, Plethodon, 12.

welleri, Plethodon, 13.

Western bull-snake, 102. racer, 94.

whip, Coach, 94.

Whip-tails, 74.

wilcoxi, Tantilla, 129.

wilderae, Eurycea, 18.

willardi, Crotalus, 137.

wislizenii, Crotaphytus, 50.

Wood-frog, 42.

Wood-turtle, 141.

woodhousei, Bufo, 30.

woodhousii, Bufo, 30 .

woodi, Sceloporus, 65.
Worm-snake, 87.

wrighti, Uta, 56 .

Xantusia, 73.

arizonae, 73.

gilberti, 73.

henshawi, 73.

riversiana, 74 .

vigilis, 74 .

Xantusiidae, 73.

Xerobates agassizii, 149.

berlandieri, 150.

Yellow rattlesnake, 134.

yonahlossee, Plethodon, 13.

yumanensis, Lampropeltis, 108.

yumensis, Lampropeltis, 108.

Zablepsis henshavii, 73.

Zamenis aurigulus, 93.

constrictor, 94.

flagellum, 94.

lateralis, $\mathbf{9 5}$.

ornatus, 96 .

piceus, 95 .

schotti, 96.

semilineatus, 96.

taeniatus, 96.

zosteromus, Sceloporus, 65. 






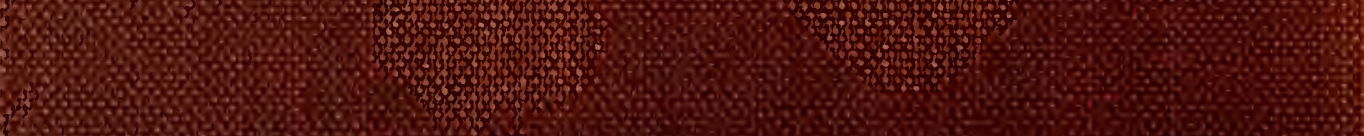

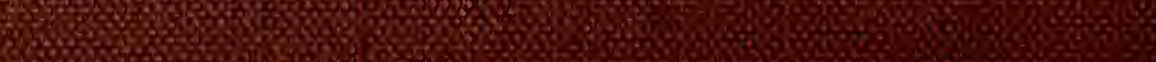

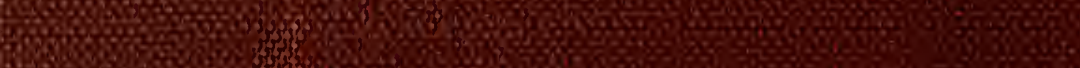

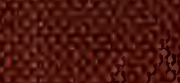

S.9.8.

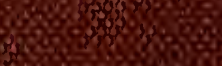

8080

and.s.

608.

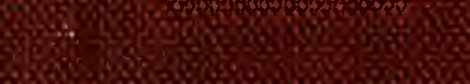

30 or 